\title{
COMPENDIUM \\ COMPENDIUM
of \\ - hIGHWAY SAFETY \\ QUESTIONNAIRE \\ ITEMS
}

Prepared for

DEPARTMENT OF TRANSPORTATION

NATIONAL HIGHWAY TRAFFIC SAFETY ADMINISTRATION

4007 th St. S.W.

Washington, D.C. 20590 


\section{PREFACE}

PAGE

55 MPH SPEED LIMIT

Attitude........................ $1-16$

Behavior .................. $17-27$

knowledge. . . . . . . . . . . . . $28-30$

ENFORCEMENT OF $55 \mathrm{MPH}$ SPEED LIMIT

Attitude......................... $32-44$

Behavior.................. 4 $45-46$

knowledge ........................ $47-48$

SAFETY BELTS AND AIRBAGS

Attitude......................... 49-62

Behavior ................. $63-70$

knowledge ........................ $71-74$

DRINKING AND DRIVINE

Attitude............... $75-79$

Behavior................ $80-84$

knowledge. ................ $85-88$

PUBLIC INFORMATION \& EDUCATION

Attitude ............................ $89-91$

knowledge .......................... $92-99$

SAFETY HELMETS

Attitude ......................... $100-101$

Behavior . . . . . . . . . . 102

Knowledge .............................. 103

PERCEPTION OF CONTROL................ 104

PERCEPTION OF RISK....................... 105

DEMOGRAPHIC . . . . . . . . . . . . $106-112$ 


\section{PREFACE}

This survey compendium contains questionnaire items and results (when furnished) that were used in state and national surveys during the period 1976 to 1980. The compendium is organized by safety issues into item groups that reflect drivers' attitudes, behaviors, and knowledge.

The compendium is periodically updated as new surveys are done. Additionally, the collection certainly does not include all state surveys that have been done, only those that are available to date.

The purpose of the compendium is to provide survey planners with a set of questionnaire items that they may wish to consider for their surveys. One should not conclude, however, that all items contained in the compendium are equally well-constructed. The benefit obtained by using an item previously employed is that one can make comparisons between states or geographic areas, or across time. Readers should also note that the compendium includes items from telephone, mail, and personal interview surveys. Not all items are suitable for every type of survey, nor would one necessarily expect comparable results if an item is used in a different survey mode.

The continued improvement and updating of the compendium depends on receiving copies of survey reports from states and other agencies engaging in surveys on highway safety. Your cooperation in this effort will ensure that the compendium is as complete and useful as possible. 


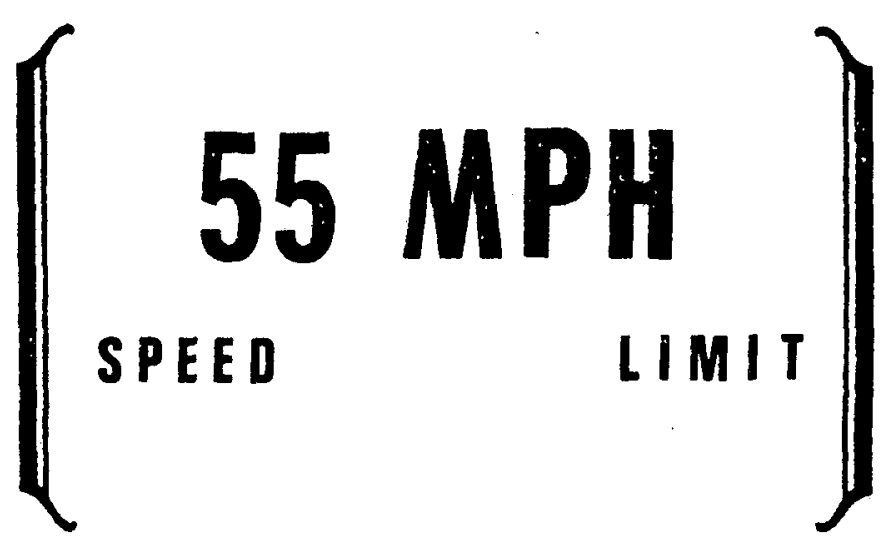




$$
\text { ATTITUDE }
$$




\begin{tabular}{|c|c|c|c|c|c|}
\hline Source & $\begin{array}{r}\text { Type of } \\
\text { Survey }\end{array}$ & Year & Sample & Question & $\%$ \\
\hline NAT & Telephone & 1978 & 1,500 & 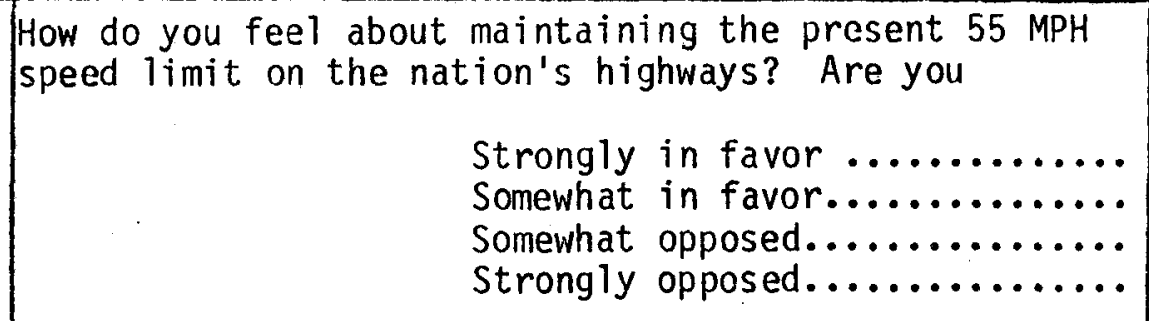 & $\begin{array}{r}55.7 \\
20.8 \\
13.4 \\
9.7\end{array}$ \\
\hline NAT & Telephone & 1979 & 1,500 & 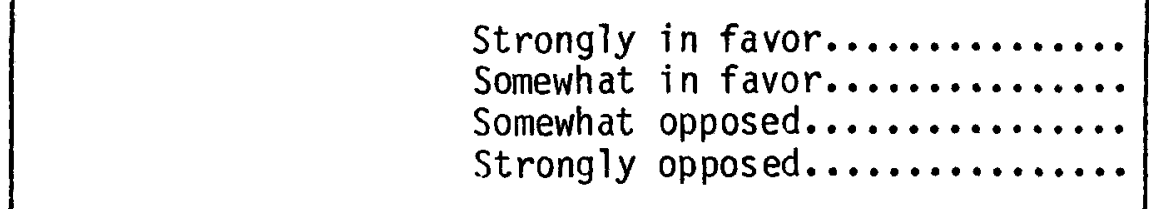 & $\begin{array}{l}57.5 \\
19.4 \\
12.5 \\
10.1\end{array}$ \\
\hline$C A$ & Personal & 1977 & 1,111 & 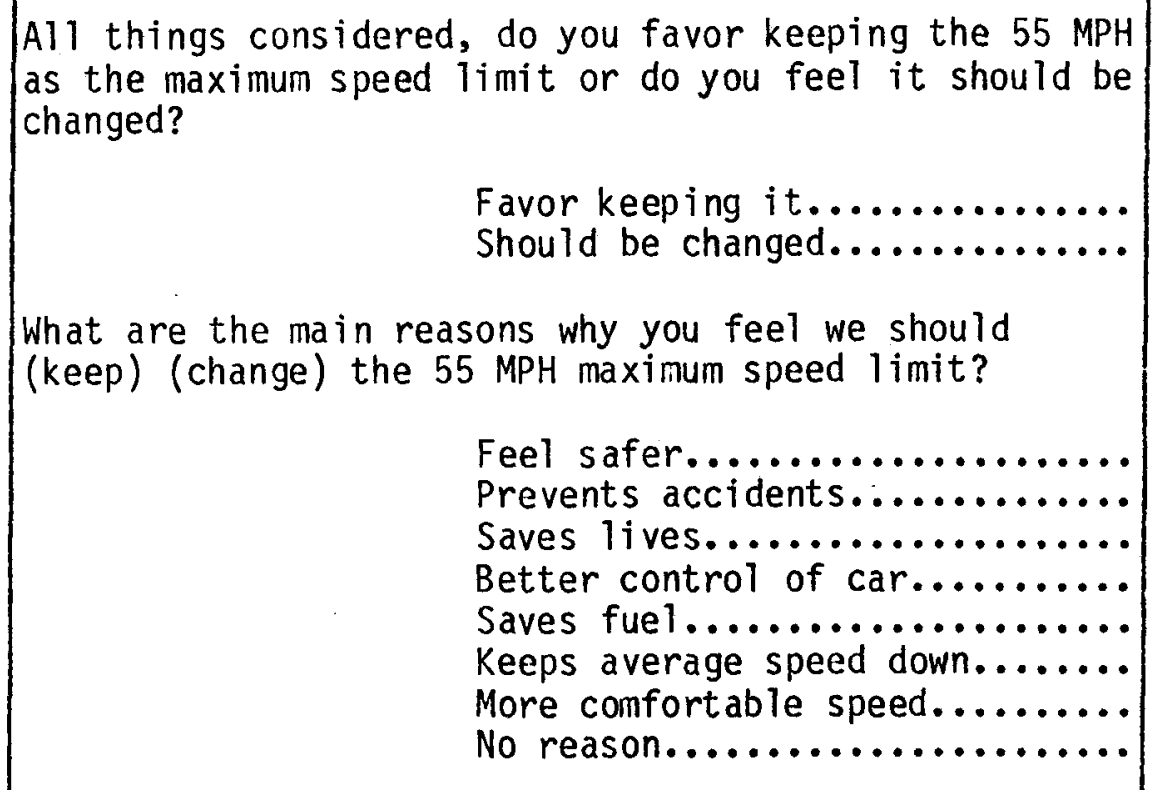 & $\begin{array}{r} \\
42.0 \\
34.0 \\
20.0 \\
10.0 \\
33.0 \\
8.0 \\
8.0 \\
3.0\end{array}$ \\
\hline AL & Personal & 1978 & 5,057 & $\begin{array}{l}\text { The } 55 \mathrm{MPH} \text { speed limit has been a law since } 1973 . \\
\text { How do you feel about it? } \\
\qquad \begin{aligned} \text { Extremely favorable.............. } \\
\text { Somewhat favorable } \\
\text { Not very favorable.............. } \\
\text { Not at all favorable }\end{aligned}\end{array}$ & $\begin{array}{l}80.0 \\
20.0\end{array}$ \\
\hline
\end{tabular}




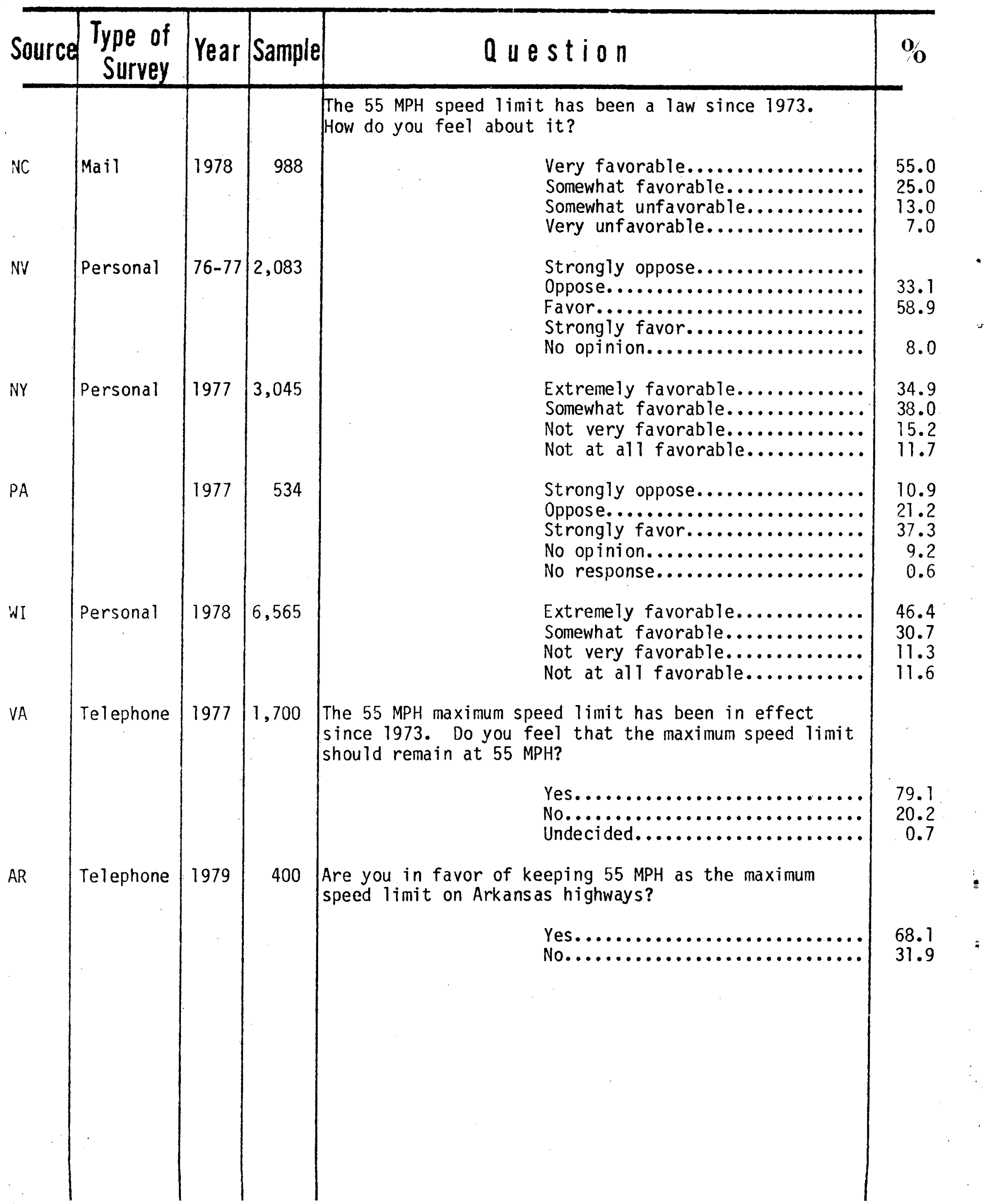




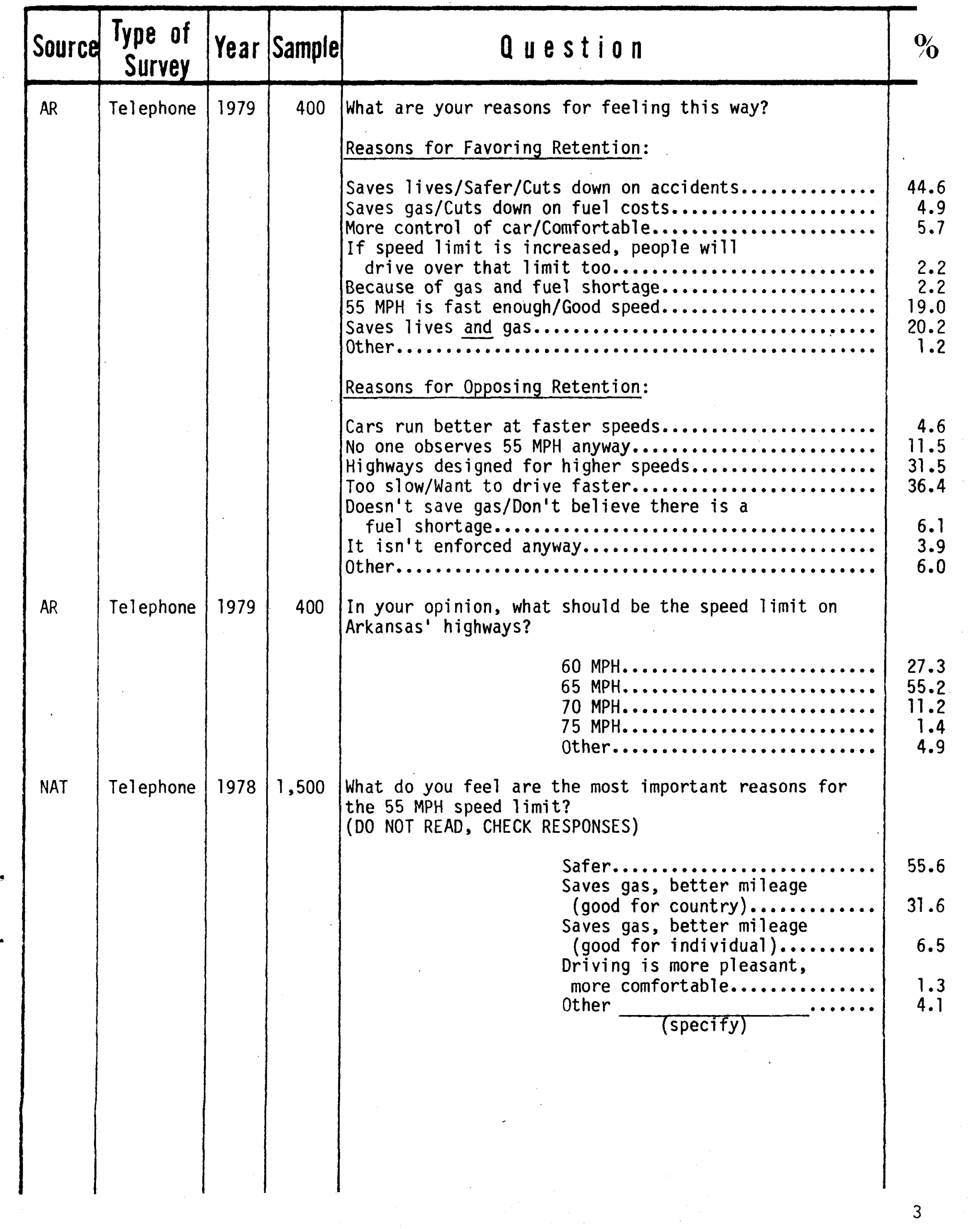




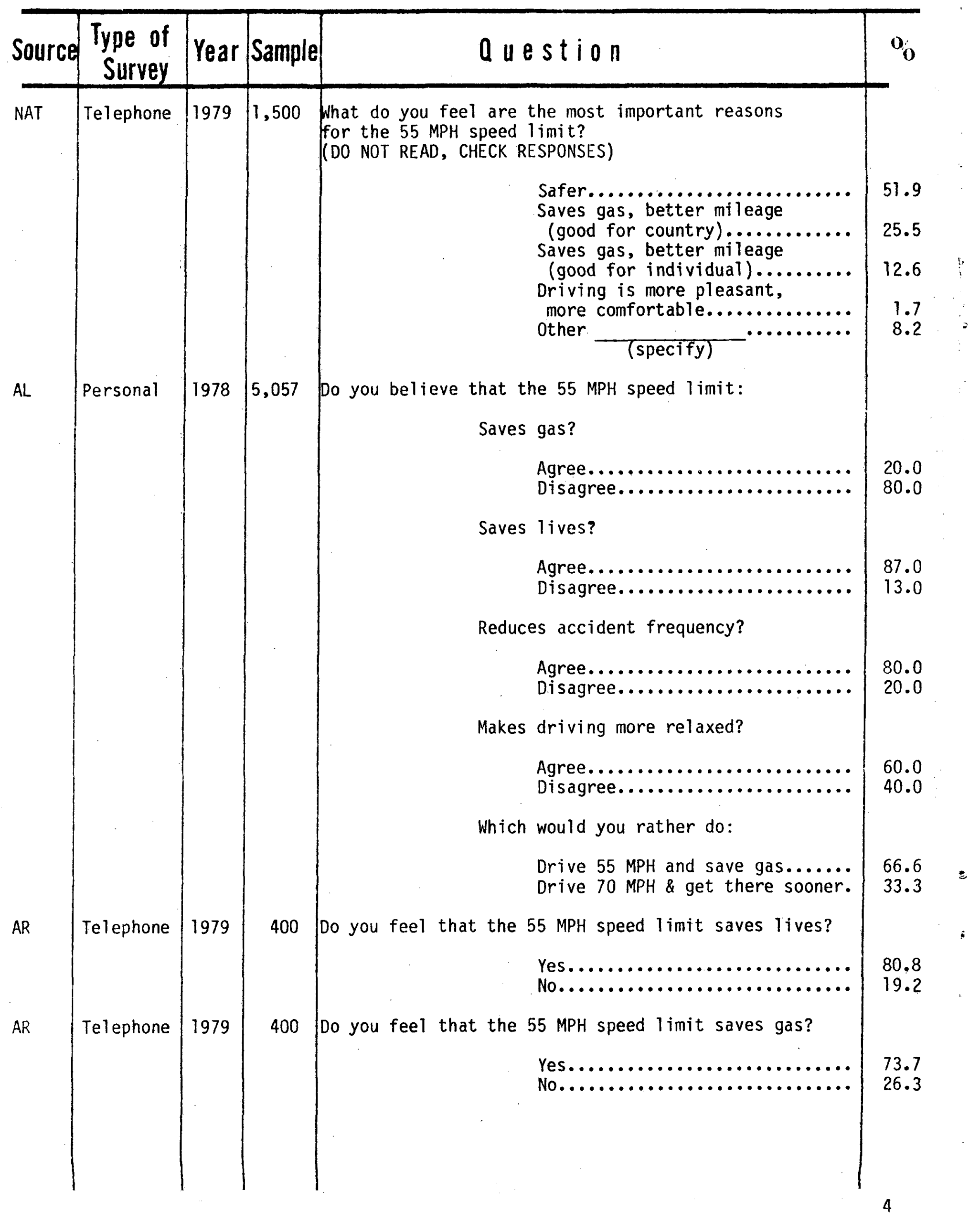




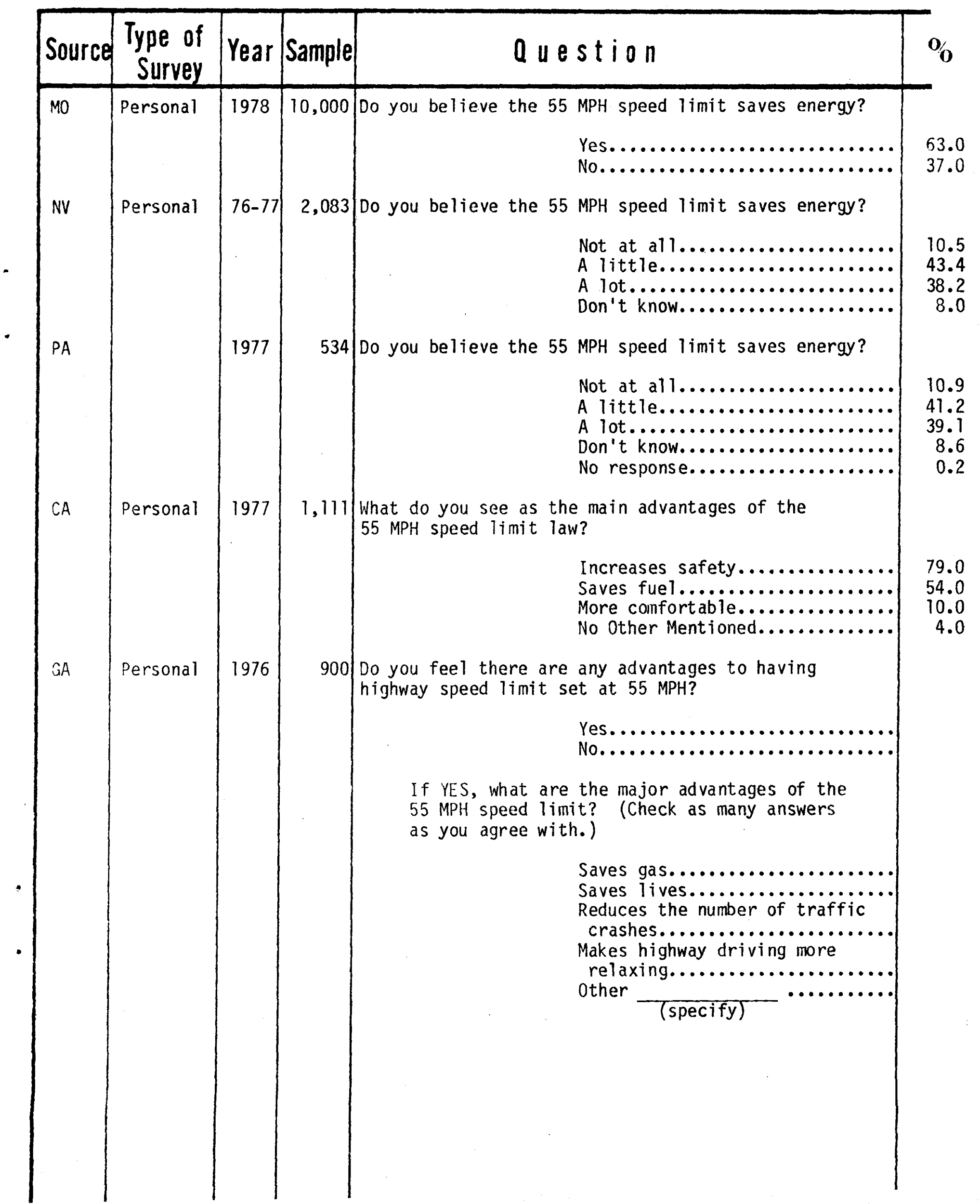




\begin{tabular}{|c|c|c|c|c|c|}
\hline Source & $\begin{array}{l}\text { Type of } \\
\text { Survey }\end{array}$ & Year & Sample & Question & 0 \\
\hline NC & Mail & 1978 & 988 & 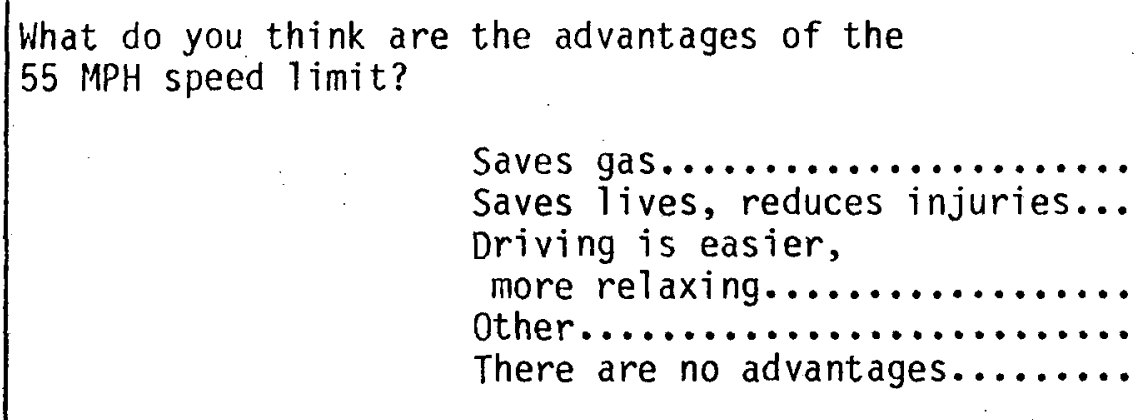 & $\begin{array}{r}34.0 \\
43.0 \\
20.0 \\
3.0 \\
0.0\end{array}$ \\
\hline PA & Telephone & 1977 & 500 & 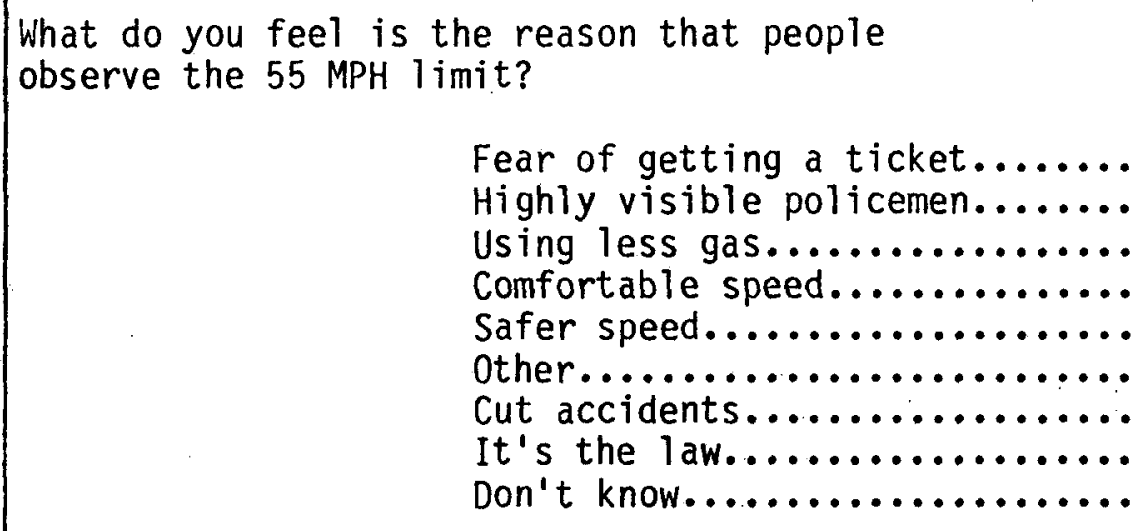 & $\begin{array}{r}25.0 \\
2.0 \\
16.0 \\
3.0 \\
45.0 \\
2.0 \\
3.0 \\
12.0 \\
4.0\end{array}$ \\
\hline$A L$ & Personal & 1978 & 5,057 & 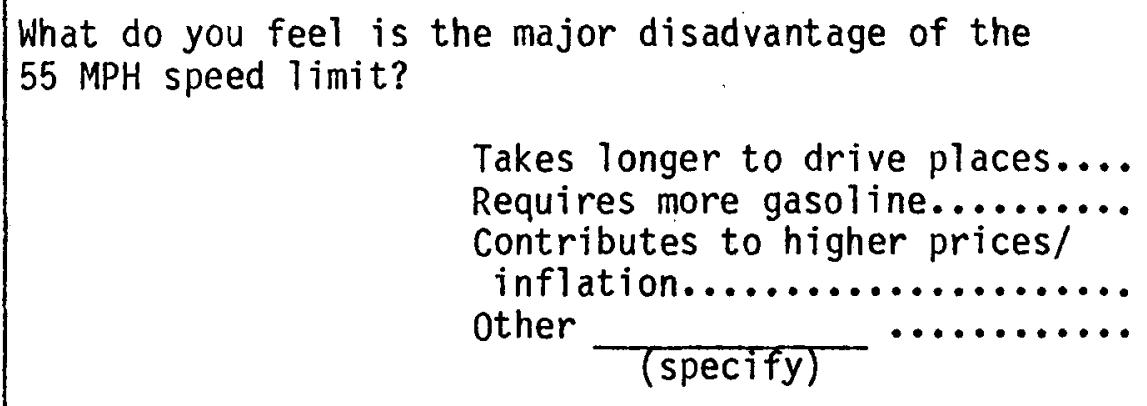 & \\
\hline$C A$ & Personal & 1977 & 1,111 & 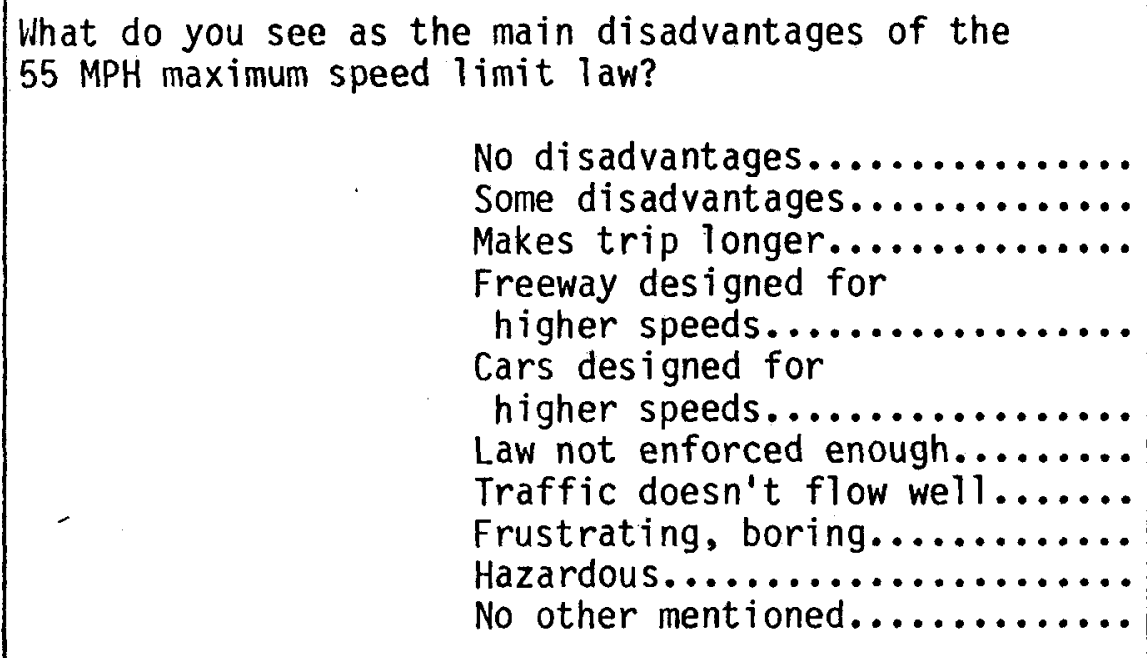 & $\begin{array}{r}32.0 \\
68.0 \\
27.0 \\
13.0 \\
7.0 \\
13.0 \\
9.0 \\
7.0 \\
5.0 \\
4.0\end{array}$ \\
\hline$G A$ & Personal & 1976 & 900 & 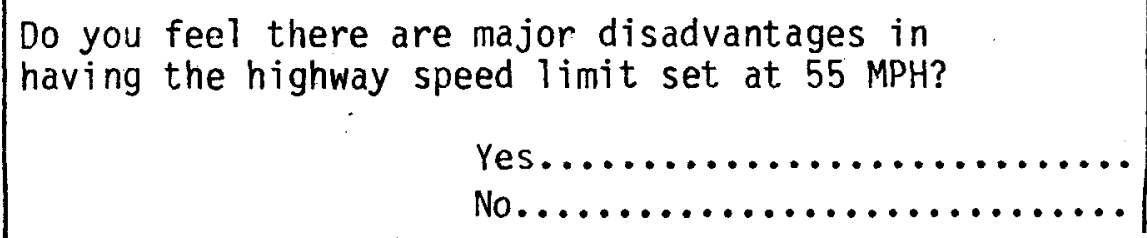 & \\
\hline
\end{tabular}




\begin{tabular}{|c|c|c|c|c|c|}
\hline Source & $\begin{array}{l}\text { Type of } \\
\text { Survey }\end{array}$ & Year & Sample & Question & $o_{0}$ \\
\hline$G A$ & $\begin{array}{l}\text { Personal } \\
\text { cont i nued) }\end{array}$ & 1976 & 900 & 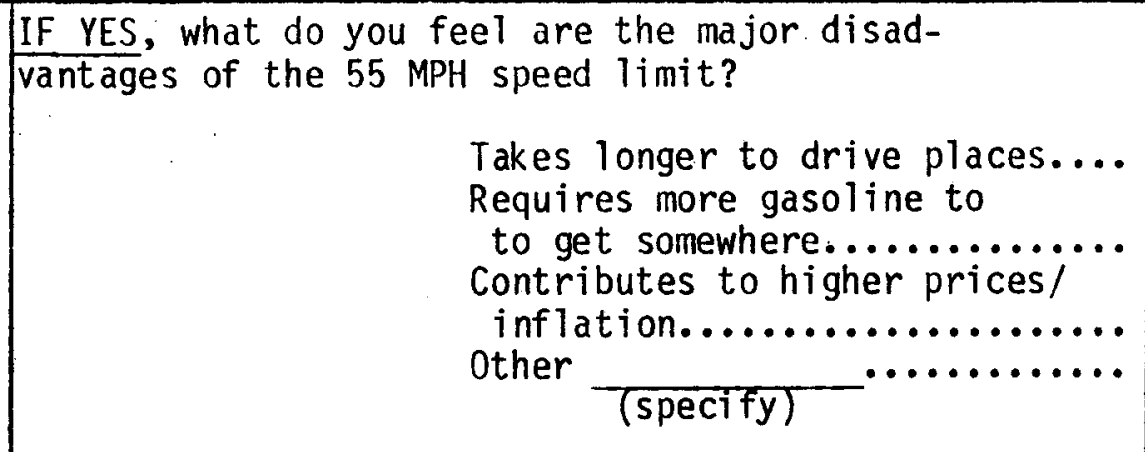 & \\
\hline NC & Mail & 1978 & 1,061 & 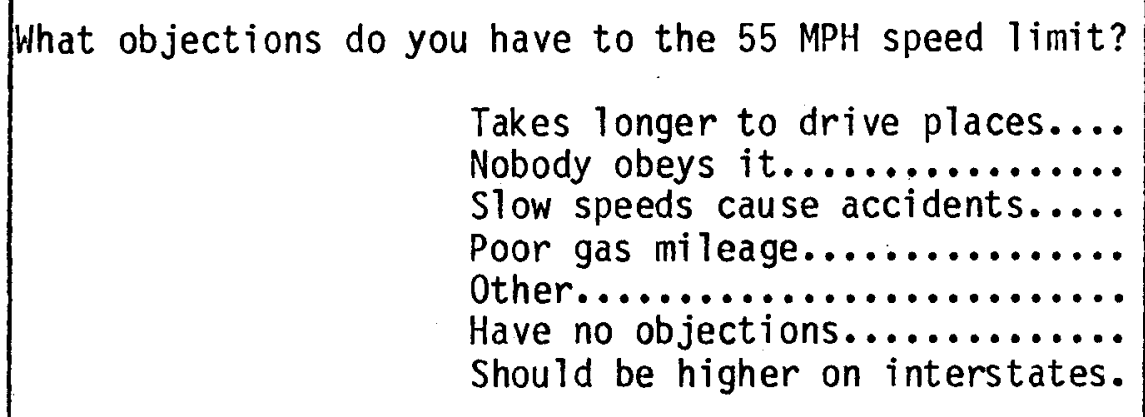 & $\begin{array}{r}29.0 \\
24.0 \\
8.0 \\
2.0 \\
3.0 \\
30.0 \\
3.0\end{array}$ \\
\hline PA & Tel ephone & 1977 & 500 & 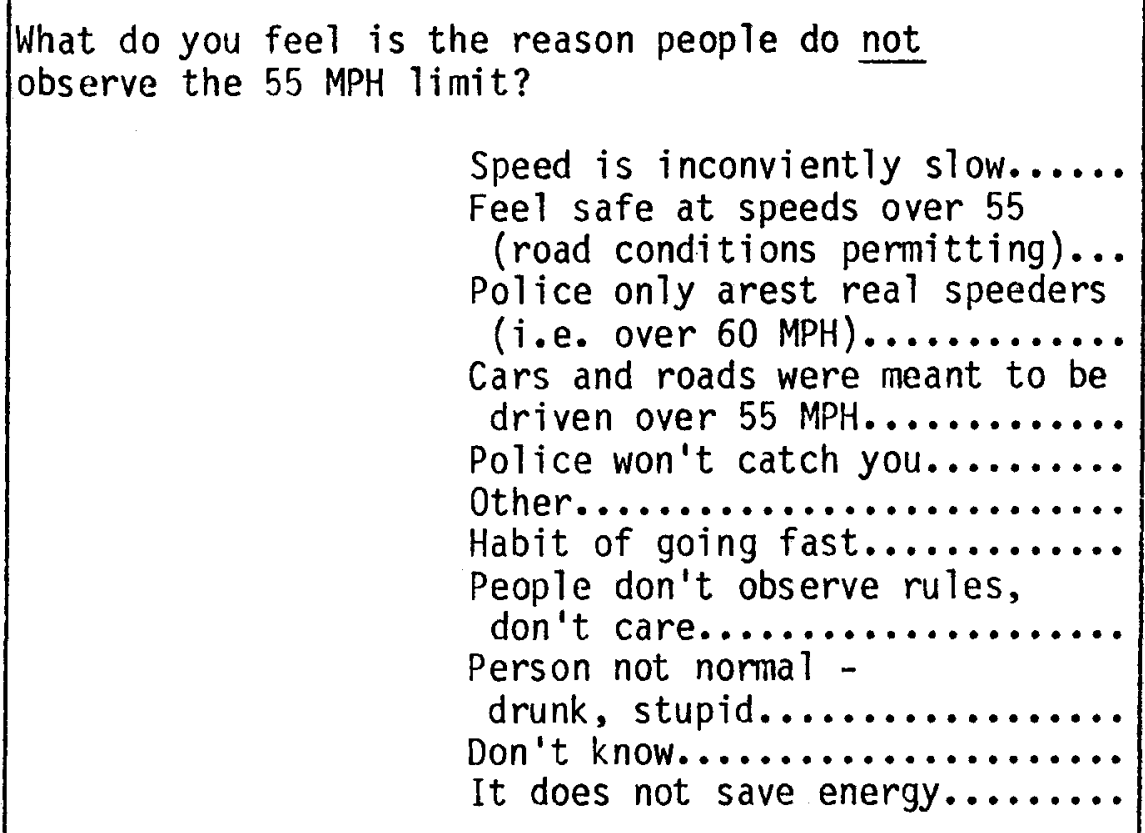 & $\begin{array}{r}50.0 \\
4.0 \\
0.0 \\
11.0 \\
2.0 \\
6.0 \\
8.0 \\
12.0 \\
10.0 \\
8.0 \\
2.0\end{array}$ \\
\hline AR & Telephone & 1979 & 400 & 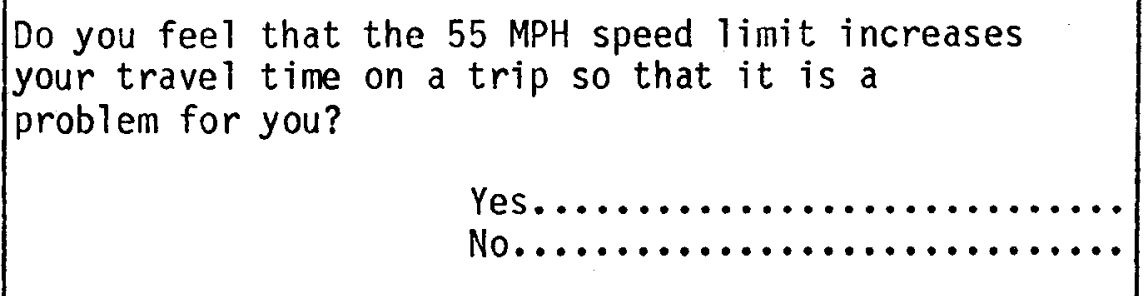 & $\begin{array}{l}31.3 \\
68.4\end{array}$ \\
\hline
\end{tabular}




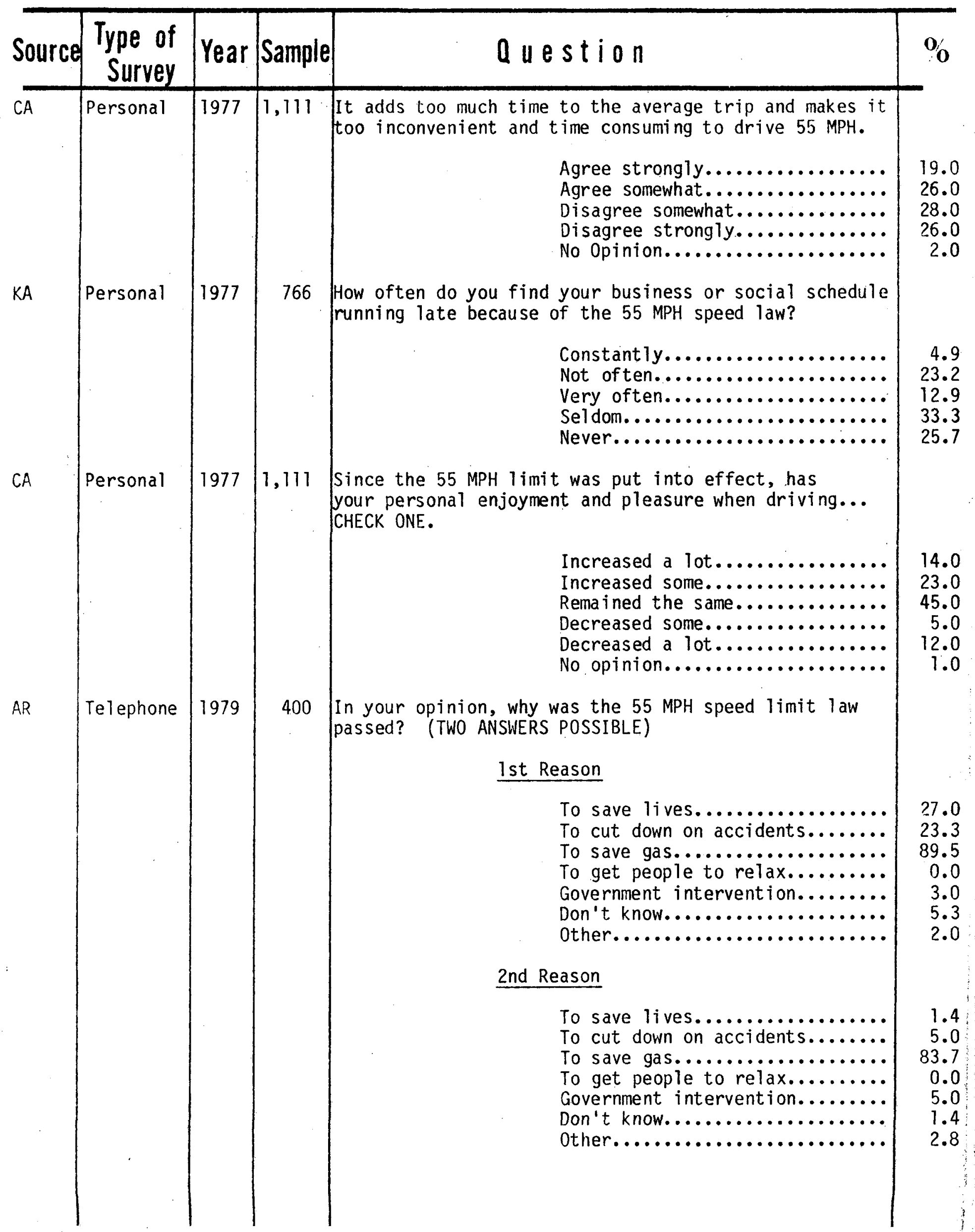




\begin{tabular}{|c|c|c|c|c|c|}
\hline Source & $\begin{array}{r}\text { Type of } \\
\text { Survey }\end{array}$ & Year & Sample & Question & \% \\
\hline AR & Telephone & 1979 & 400 & 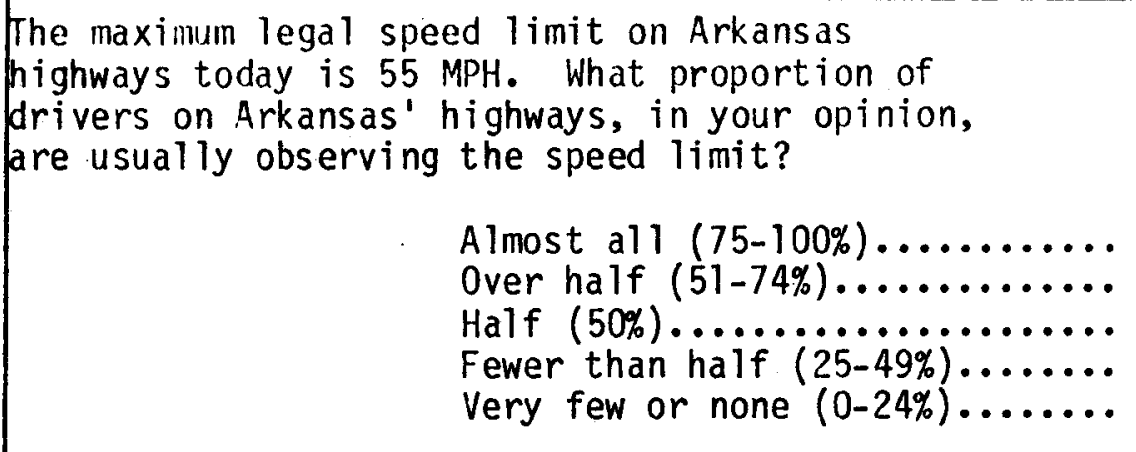 & $\begin{array}{l}10.3 \\
12.3 \\
28.0 \\
26.7 \\
22.2\end{array}$ \\
\hline$C A$ & Personal & 1977 & 1,111 & 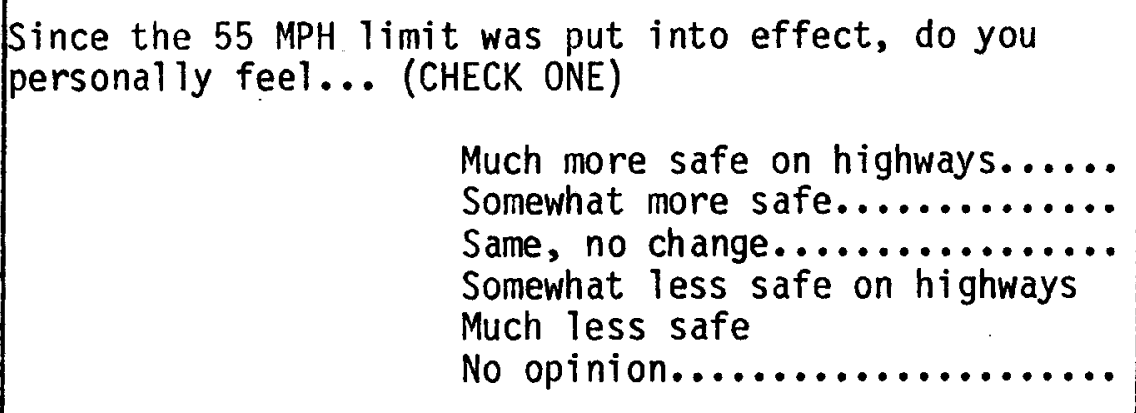 & $\begin{array}{r}22.0 \\
42.0 \\
28.0 \\
7.0 \\
1.0\end{array}$ \\
\hline$C A$ & Personal & 1977 & 1,111 & 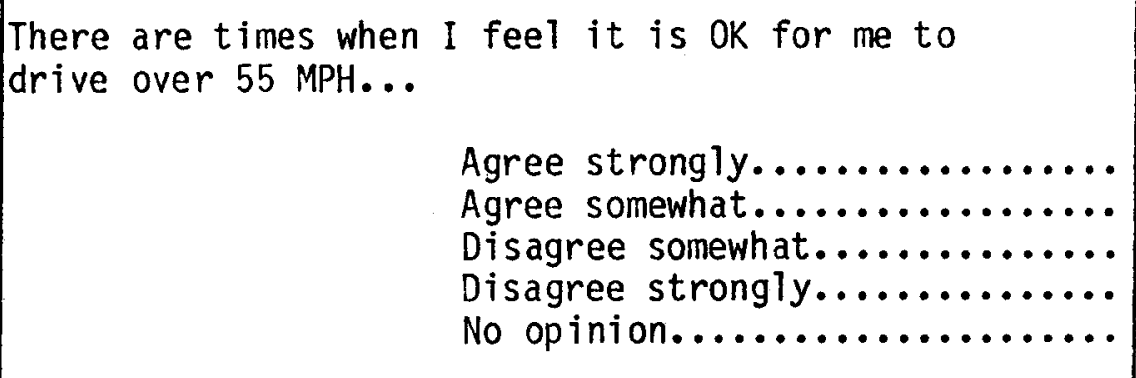 & $\begin{array}{r}28.0 \\
44.0 \\
12.0 \\
15.0 \\
1.0\end{array}$ \\
\hline$C A$ & Personal & 1977 & 1,111 & 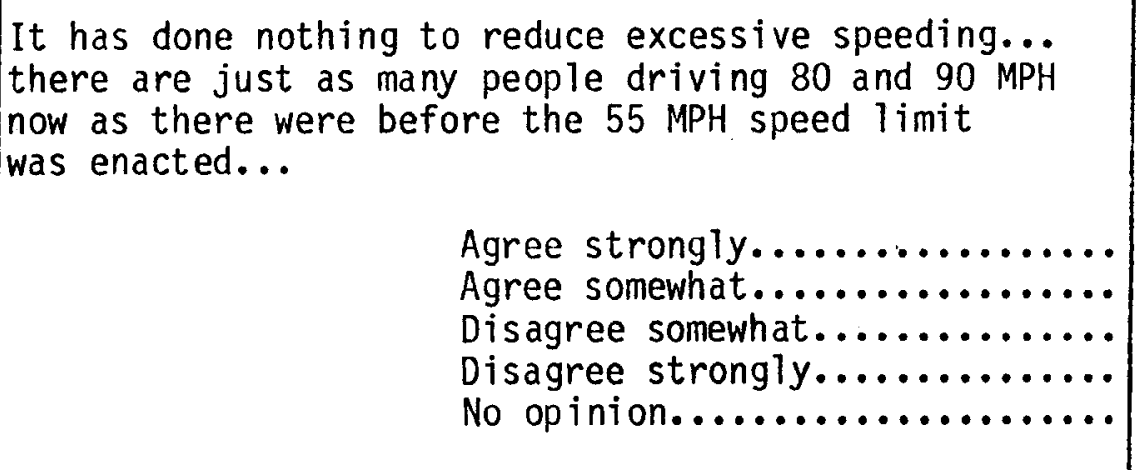 & $\begin{array}{r}12.0 \\
22.0 \\
37.0 \\
27.0 \\
2.0\end{array}$ \\
\hline$C A$ & Personal & 1977 & 1,111 & 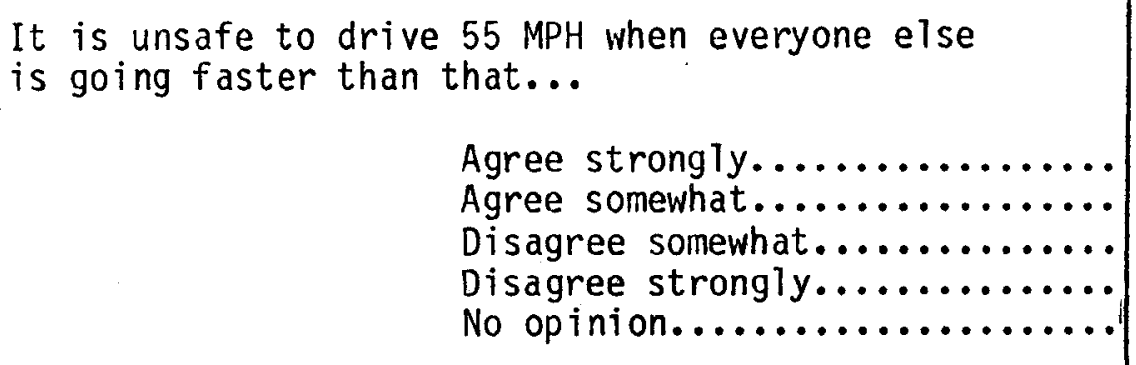 & $\begin{array}{r}24.0 \\
35.0 \\
24.0 \\
15.0 \\
2.0\end{array}$ \\
\hline
\end{tabular}




\begin{tabular}{|c|c|c|c|c|}
\hline Source & $\begin{array}{l}\text { Type of } \\
\text { Survey }\end{array}$ & Year & Sample & Question \\
\hline$C A$ & Personal & 1977 & 1,111 & 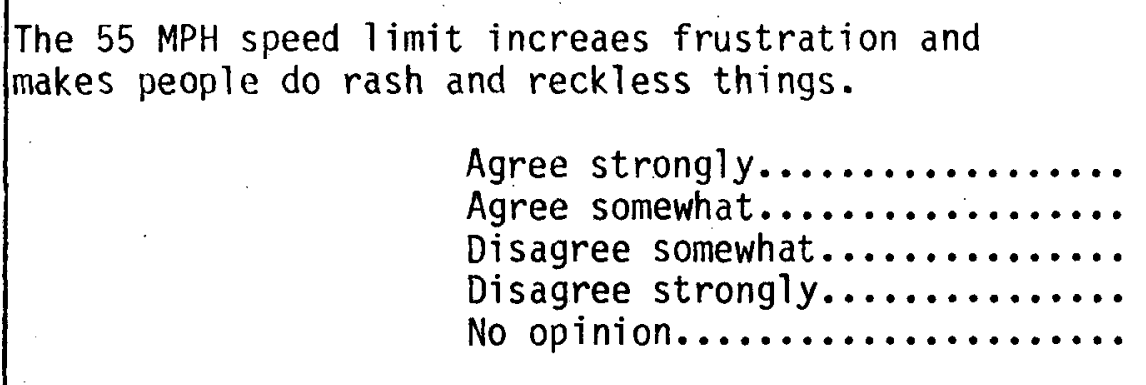 \\
\hline$C A$ & Personal & 1977 & 1,111 & 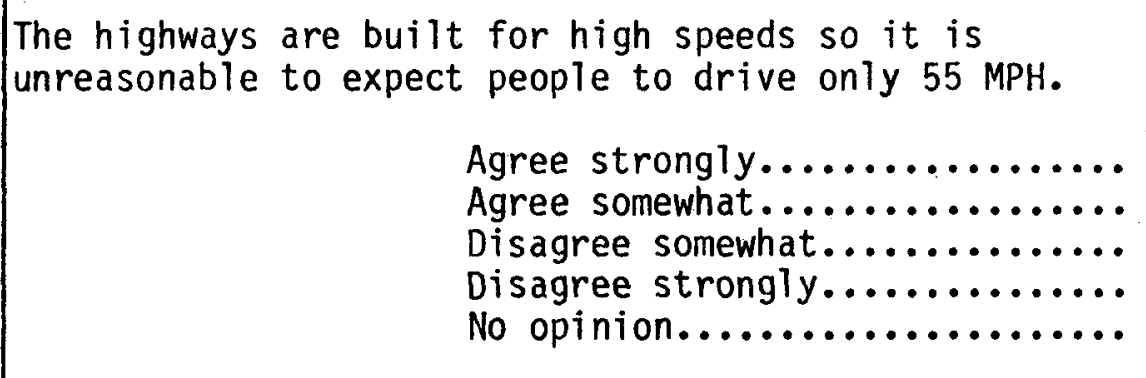 \\
\hline IA & Telephone & 1978 & 605 & 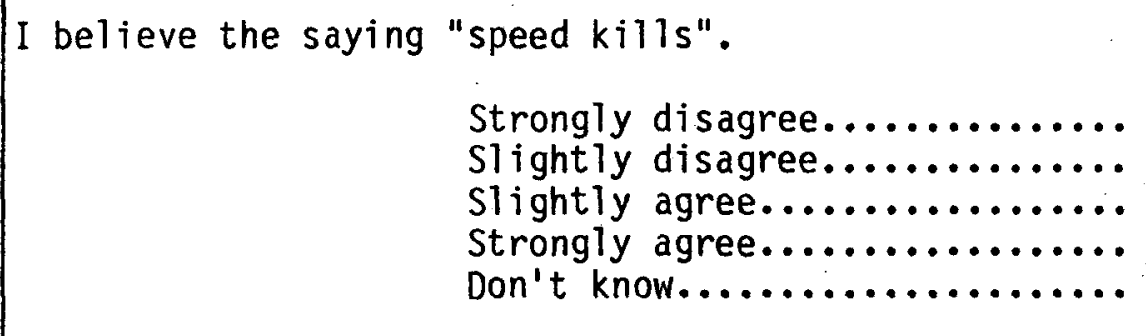 \\
\hline$A R$ & Telephone & 1979 & 400 & 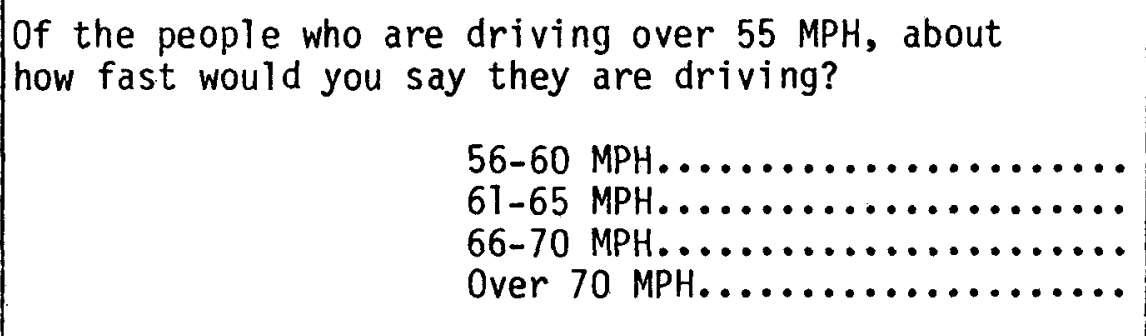 \\
\hline KA & Personal & 1977 & 766 & 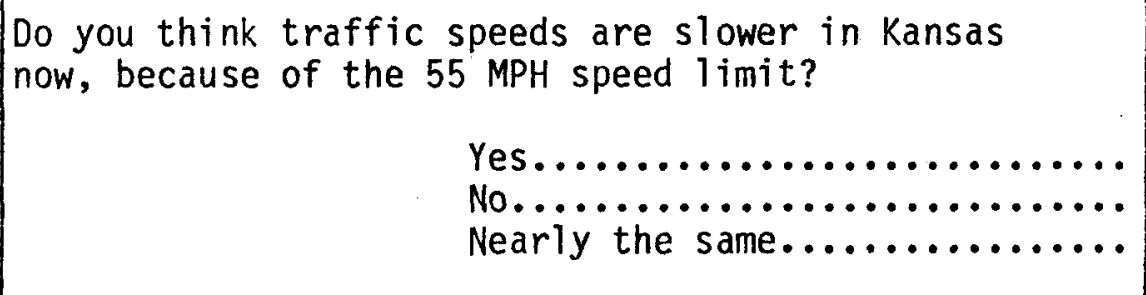 \\
\hline$A L$ & Personal & 1978 & 5,057 & 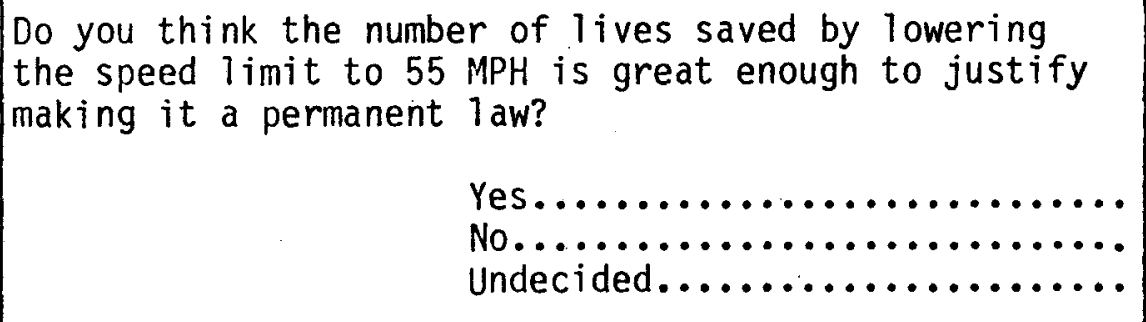 \\
\hline
\end{tabular}




\begin{tabular}{|c|c|c|c|c|c|}
\hline Source & $\begin{array}{l}\text { Type of } \\
\text { Survey }\end{array}$ & Year & Sample & Question & $0_{0}$ \\
\hline $\mathrm{GA}$ & Personal & 1976 & 900 & 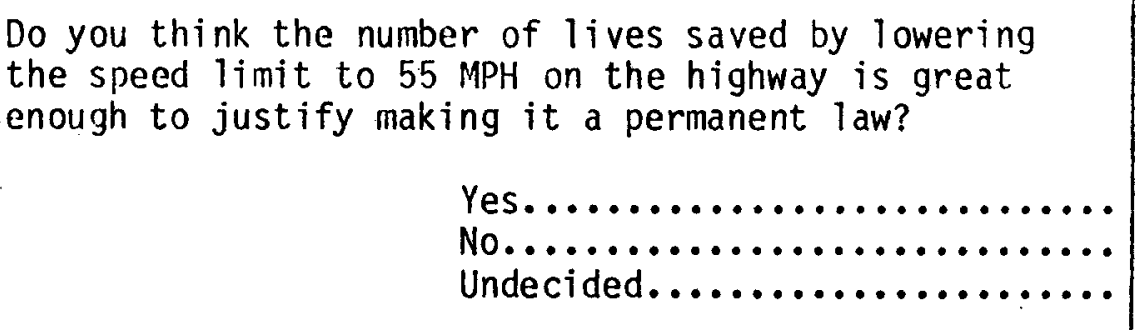 & \\
\hline KA & Personal & 1977 & 776 & 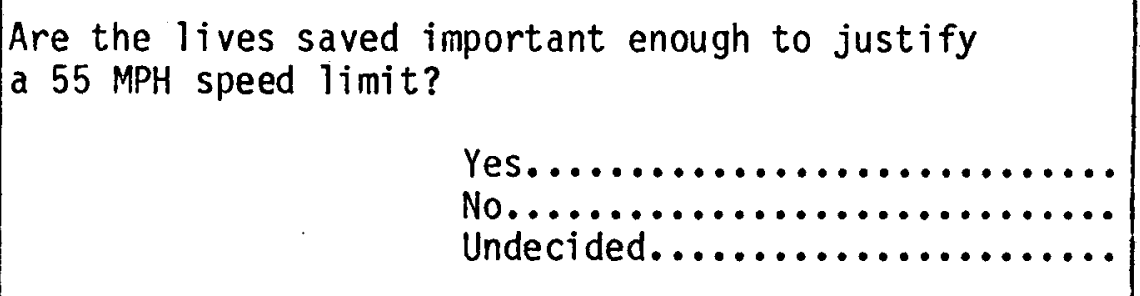 & $\begin{array}{r}76.0 \\
8.4 \\
15.6\end{array}$ \\
\hline$A R$ & Tel ephone & 1979 & 400 & 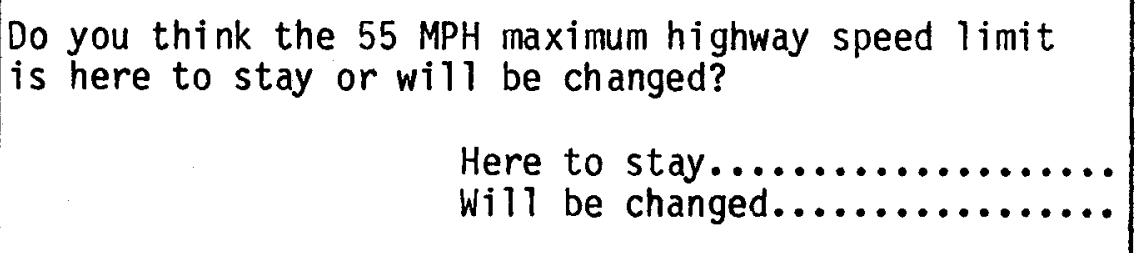 & $\begin{array}{l}56.1 \\
43.9\end{array}$ \\
\hline IA & Telephone & 1978 & 605 & 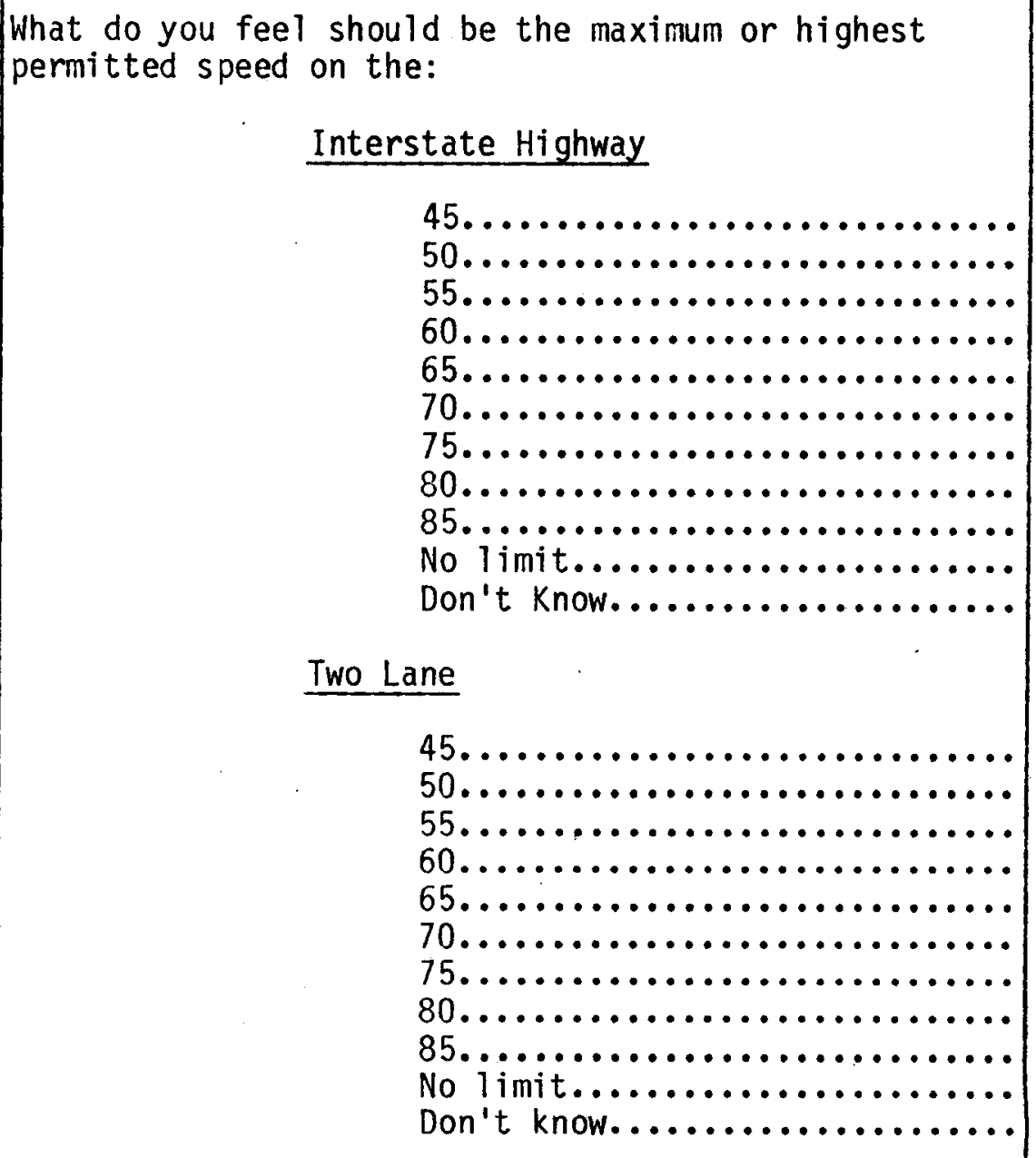 & $\begin{array}{r}0.0 \\
0.5 \\
32.7 \\
26.1 \\
24.4 \\
9.9 \\
4.4 \\
1.0 \\
0.0 \\
0.5 \\
0.5 \\
\\
\\
0.5 \\
6.0 \\
62.1 \\
19.5 \\
9.4 \\
1.0 \\
0.0 \\
0.0 \\
0.0 \\
0.5 \\
0.0\end{array}$ \\
\hline
\end{tabular}




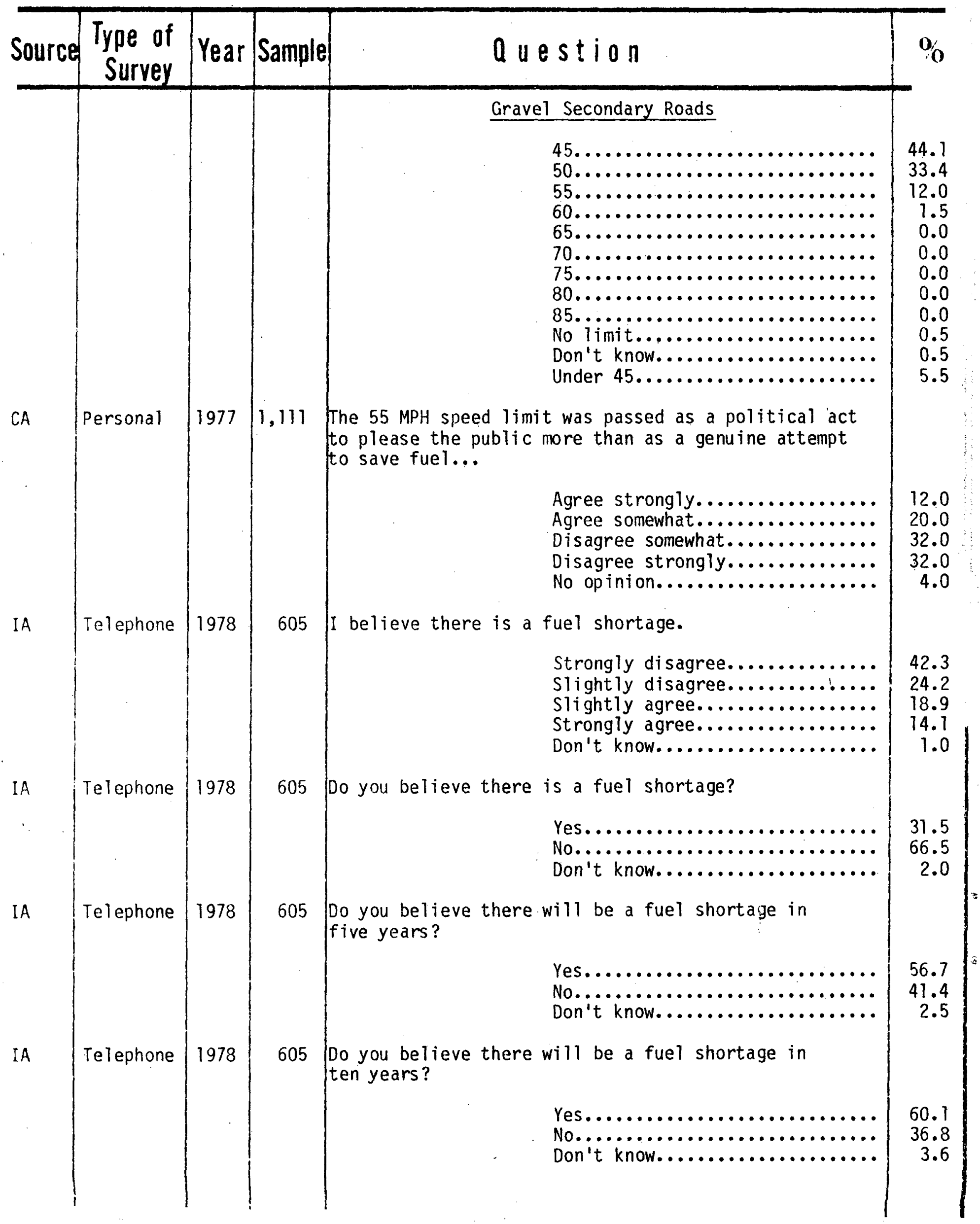




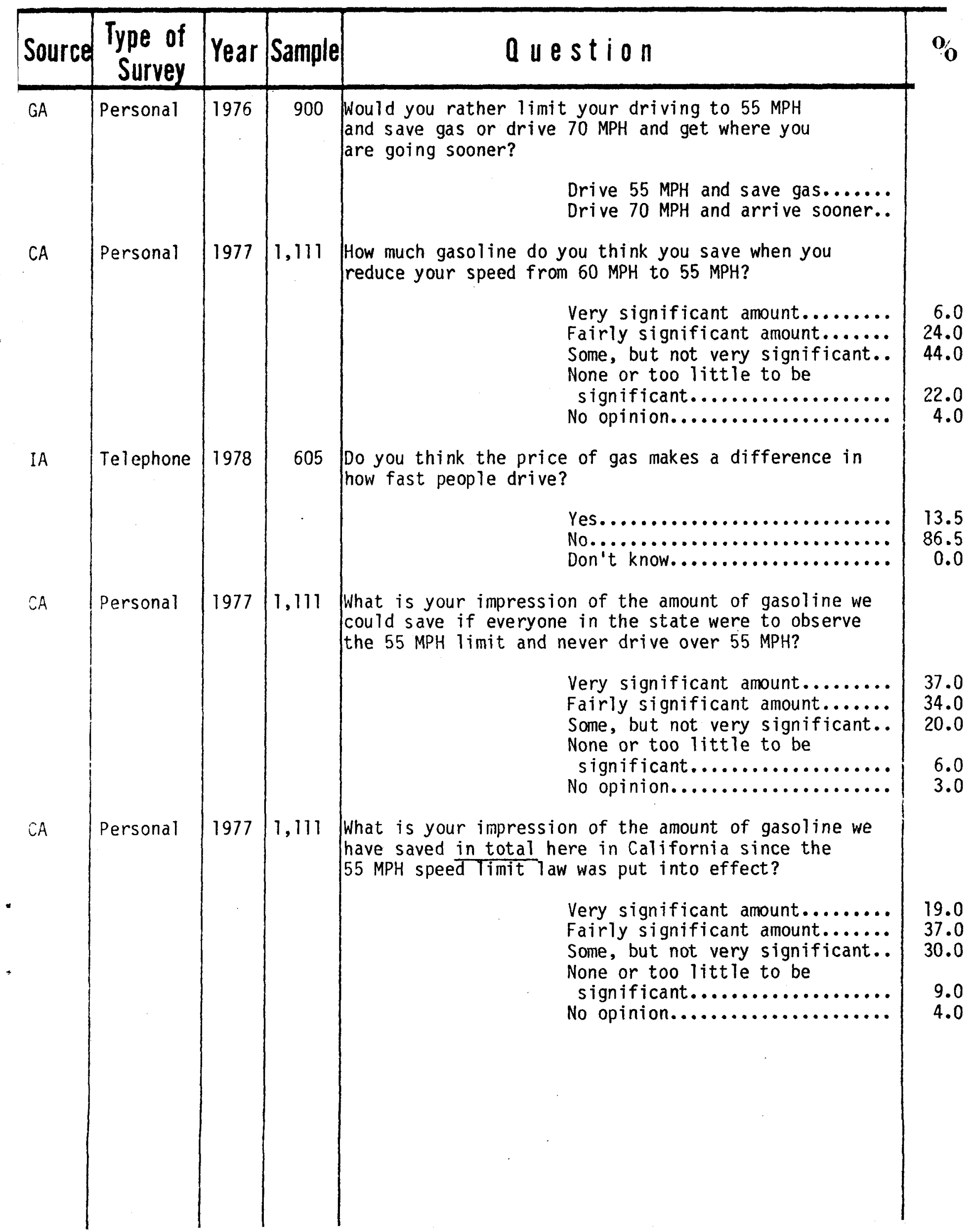




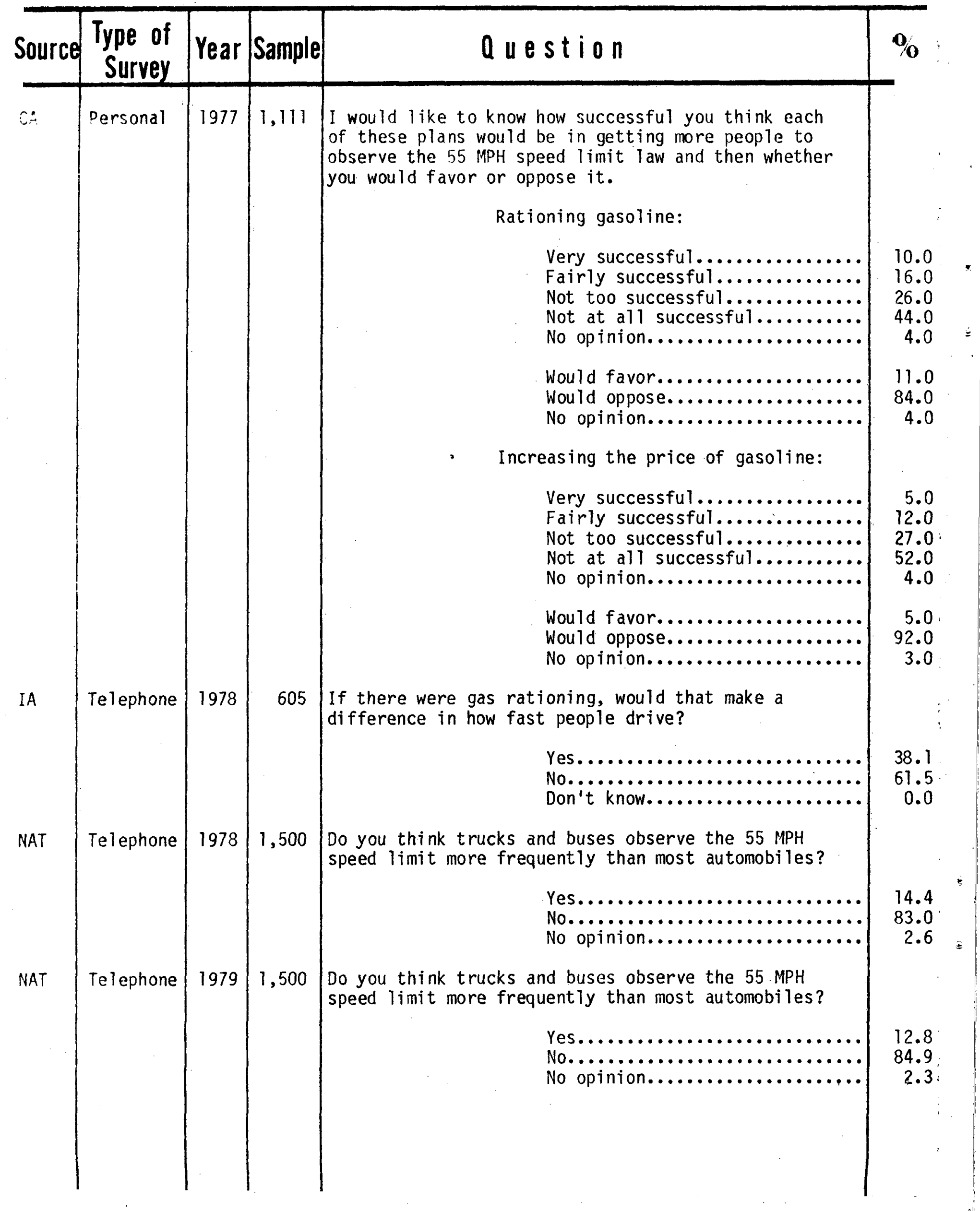




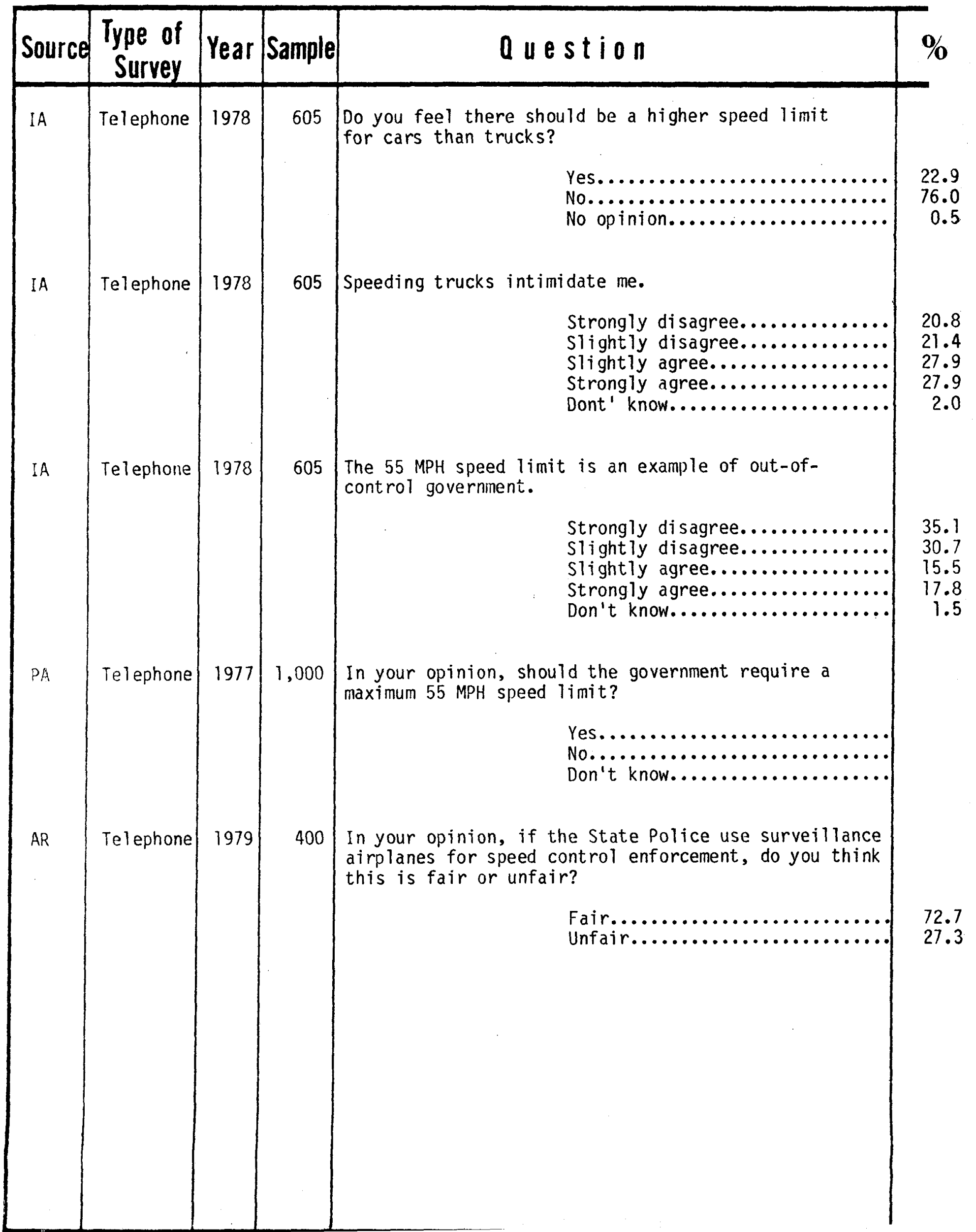




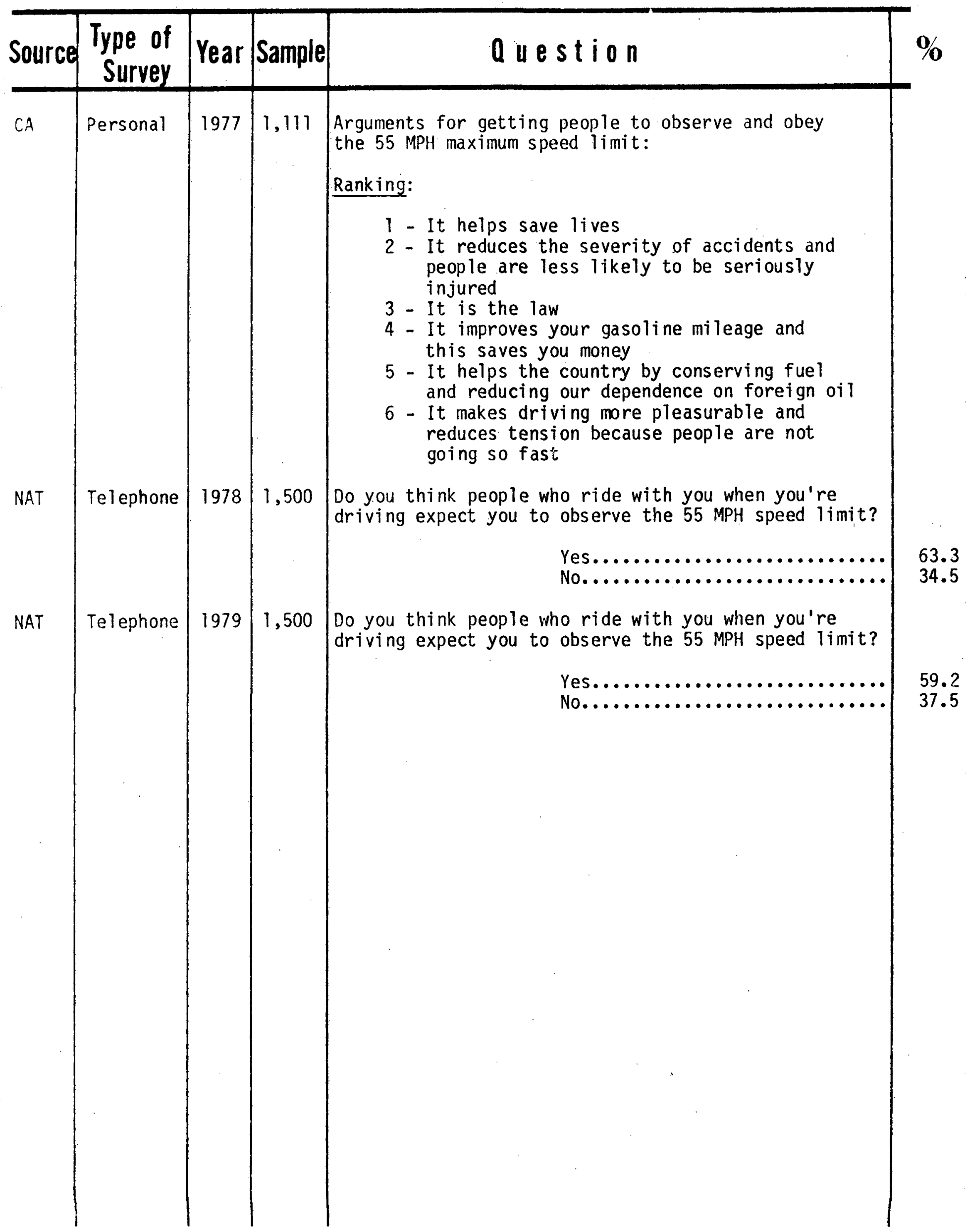

63.3

34.5

59.2

37.5 
BEHANIOR 


\begin{tabular}{|c|c|c|c|c|c|}
\hline Source & $\begin{array}{r}\text { Type of } \\
\text { Survey }\end{array}$ & Year & Sample & Question & $\%$ \\
\hline NAT & Tel ephone & 1978 & 1,500 & 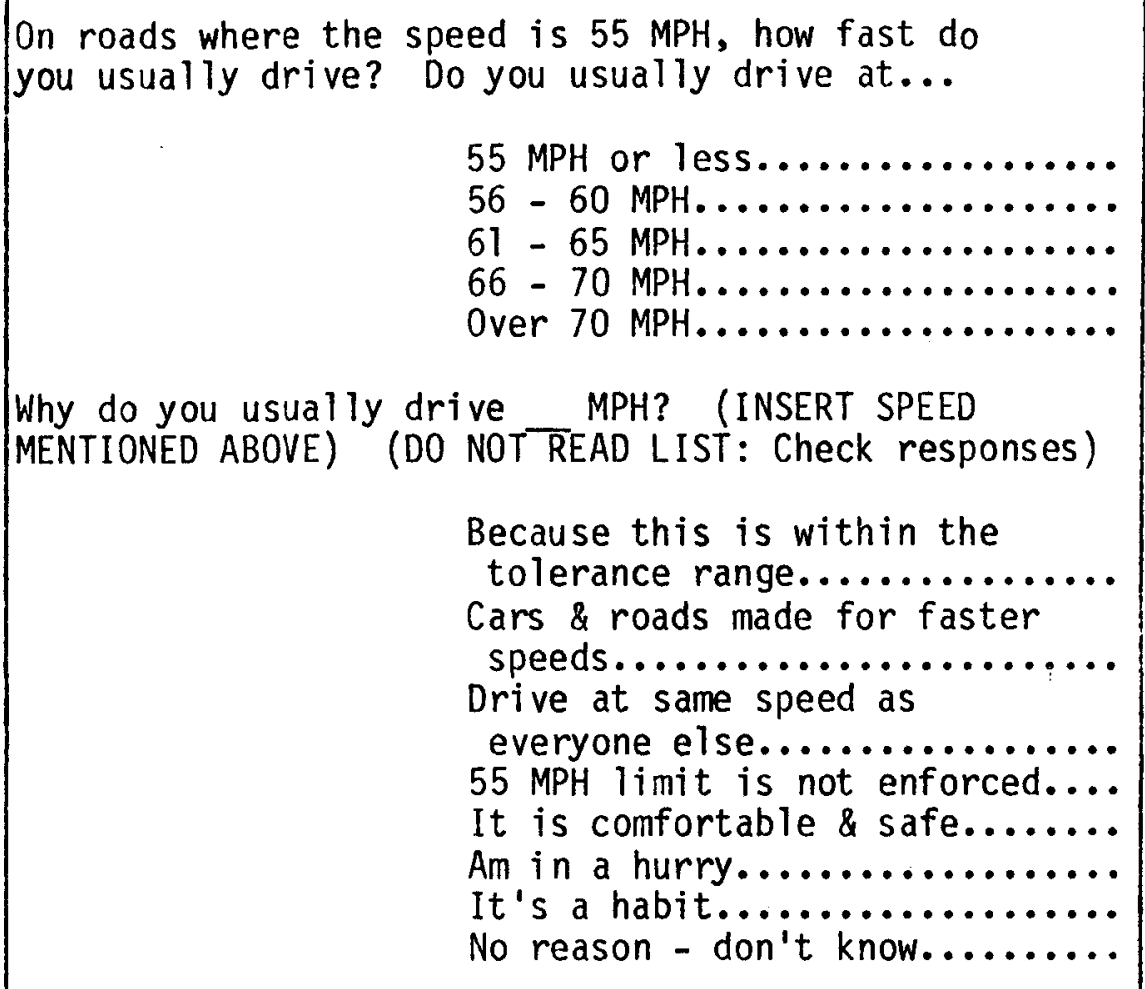 & $\begin{array}{r}20.3 \\
15.6 \\
22.6 \\
2.6 \\
15.3 \\
11.3 \\
9.9 \\
2.4\end{array}$ \\
\hline NAT & Telephone & 1979 & 1,500 & 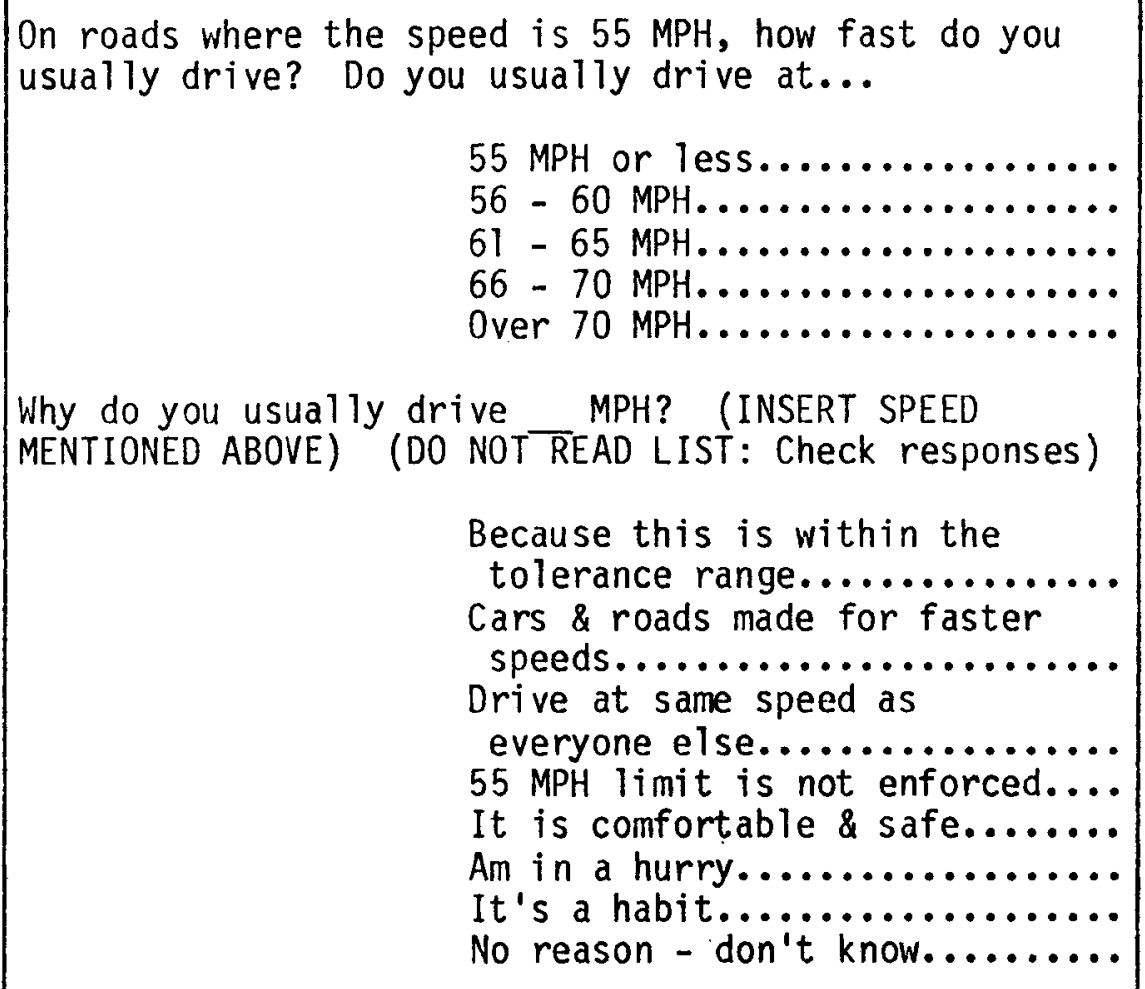 & $\begin{array}{r} \\
52.4 \\
37.4 \\
7.1 \\
2.3 \\
0.3 \\
\\
\\
\\
13.9 \\
13.0 \\
18.9 \\
2.4 \\
24.6 \\
11.1 \\
9.9 \\
6.2\end{array}$ \\
\hline
\end{tabular}




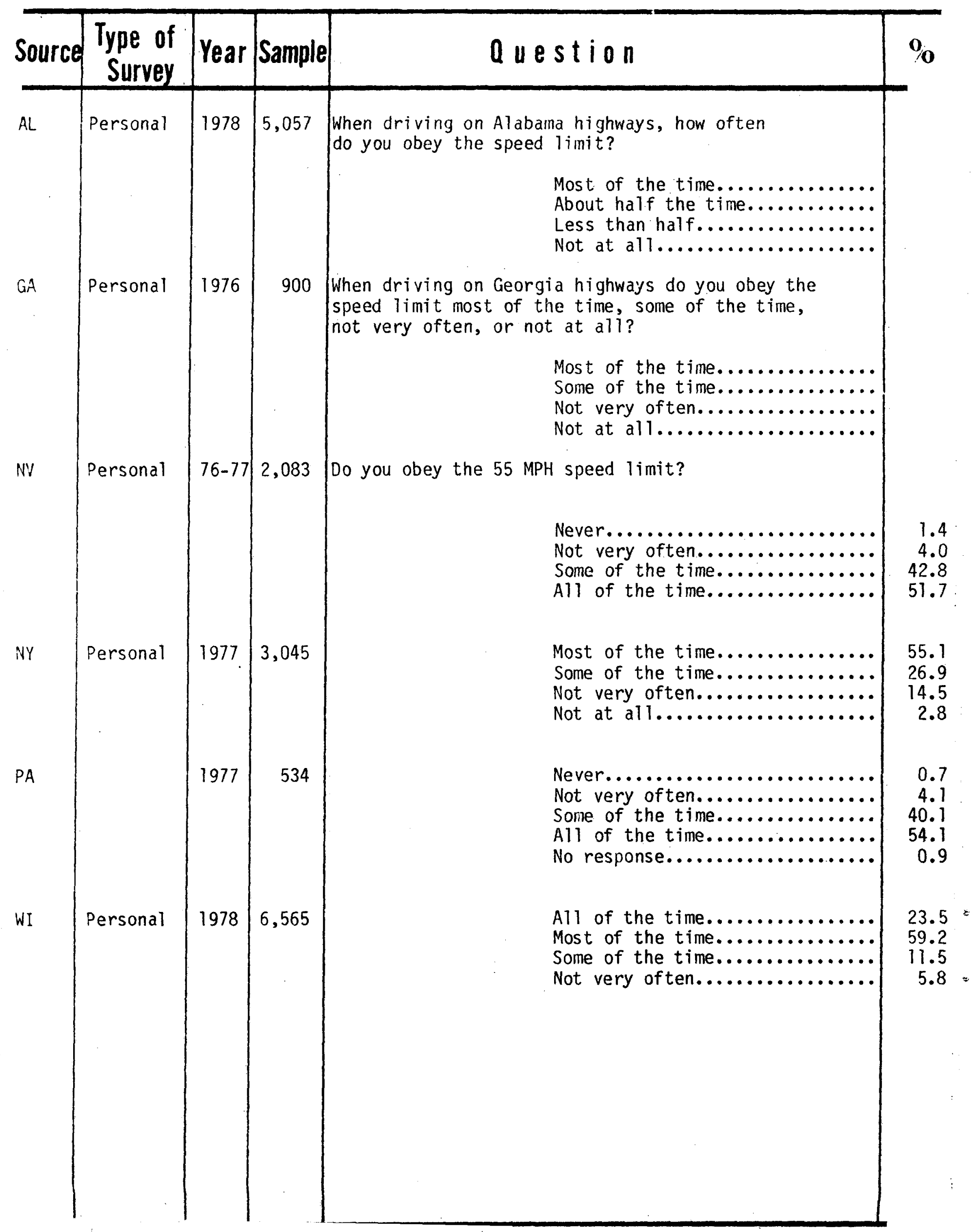




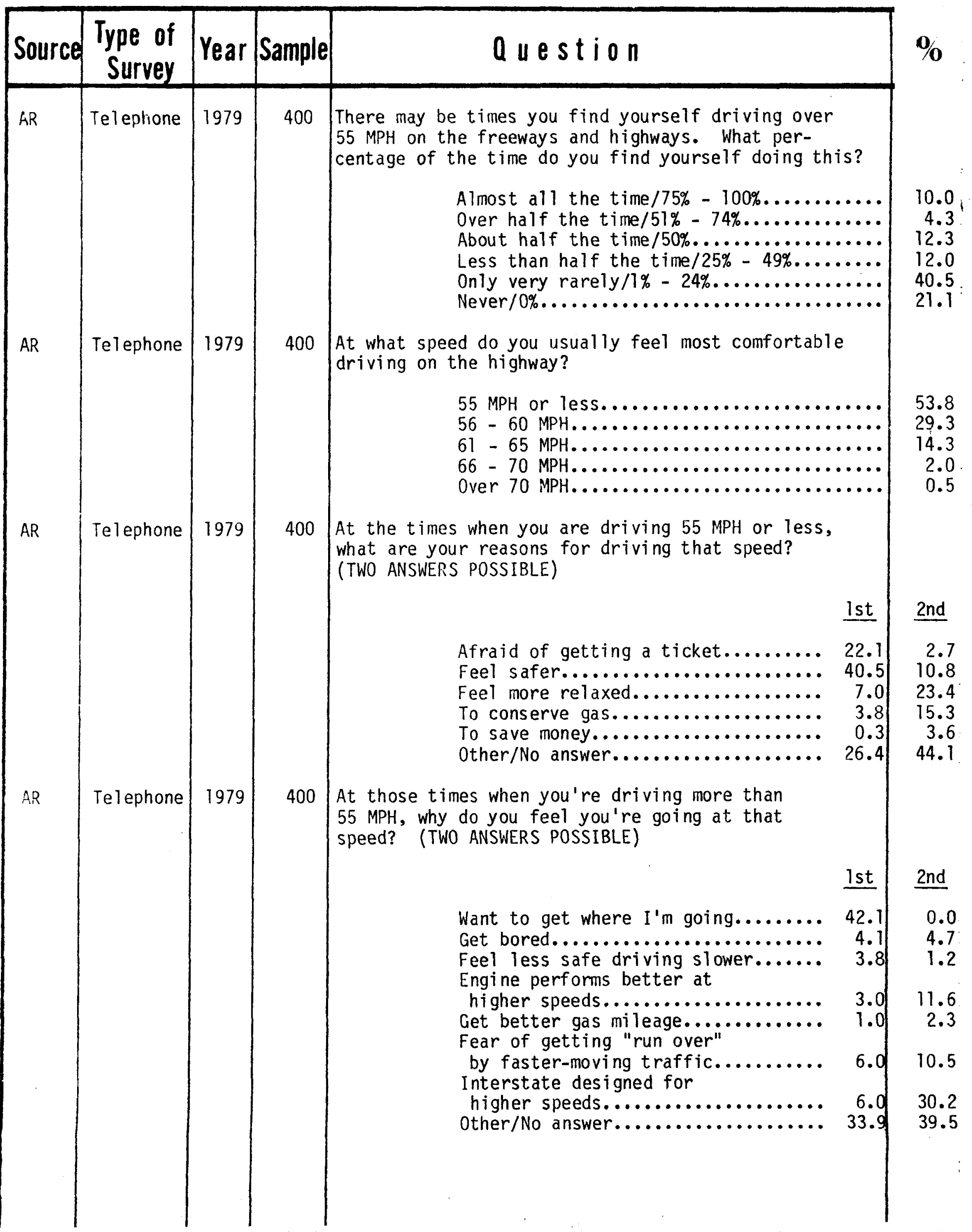




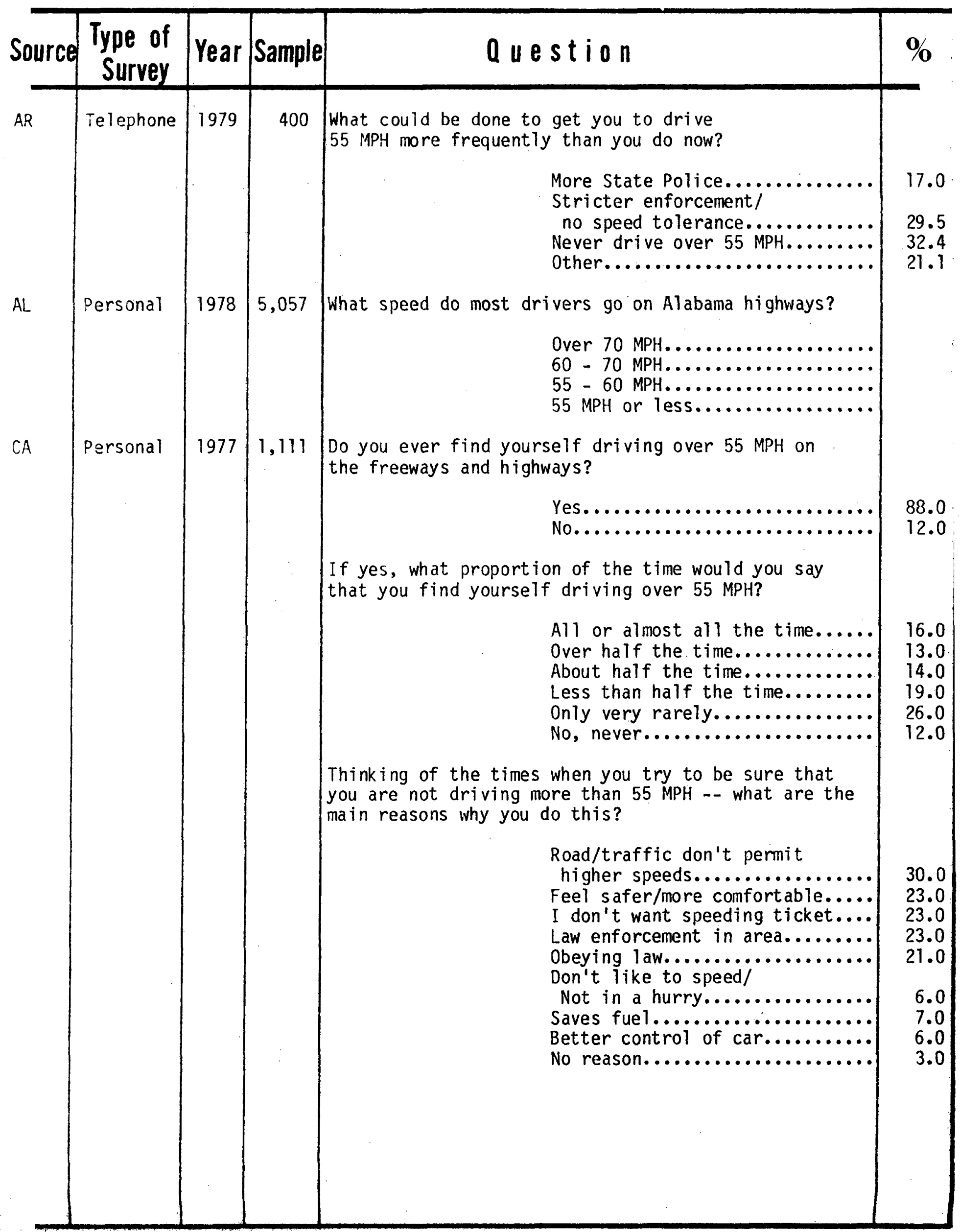




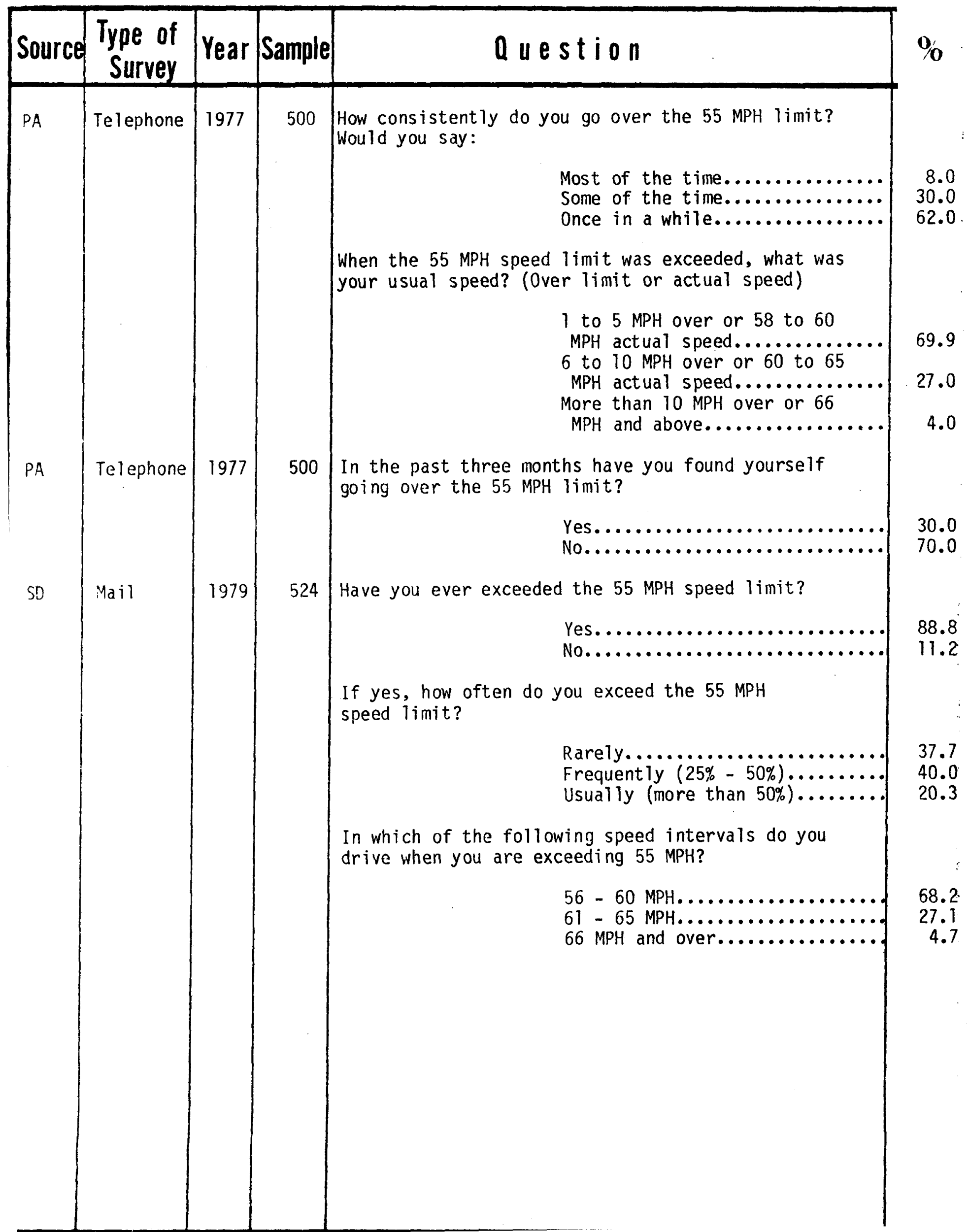




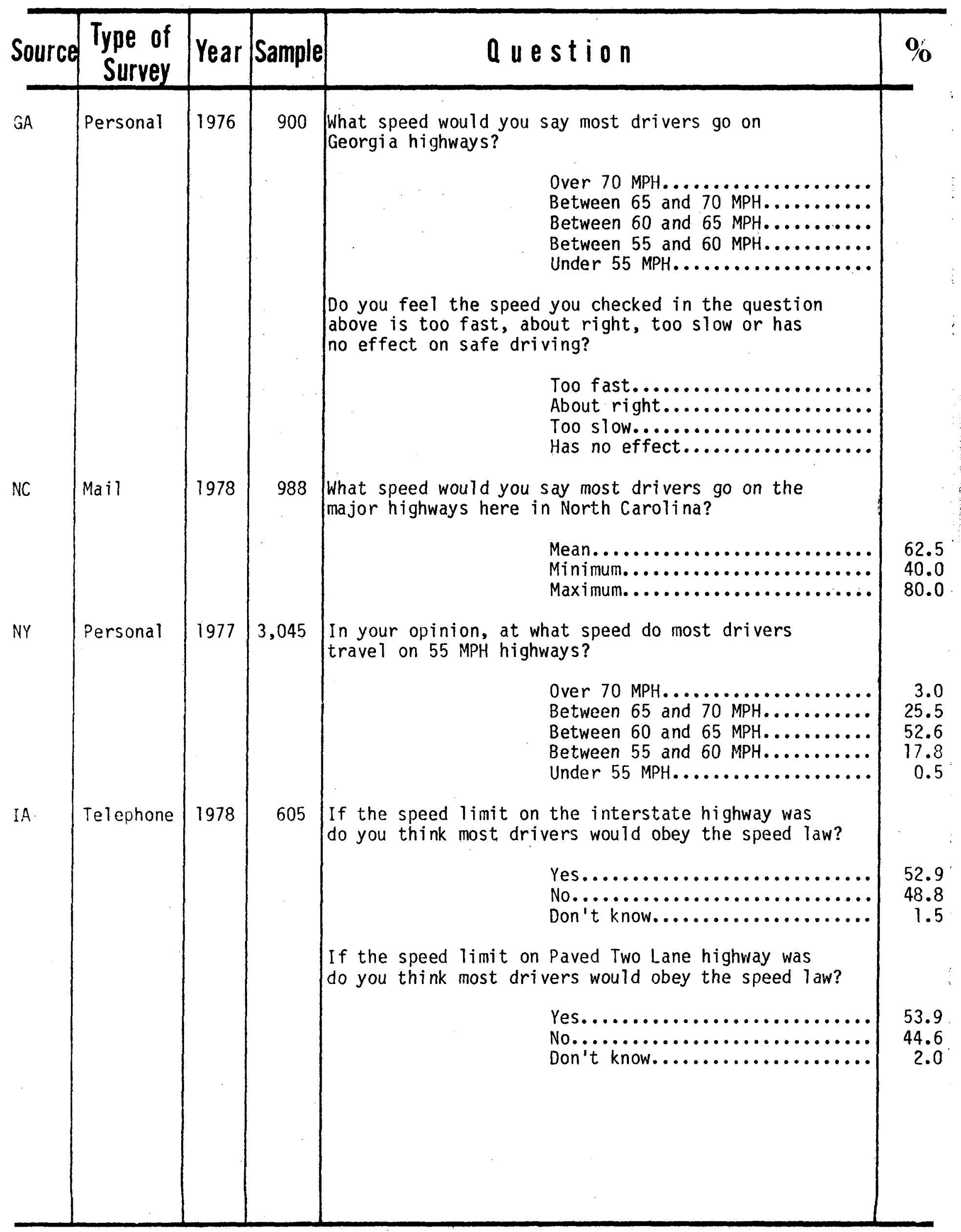




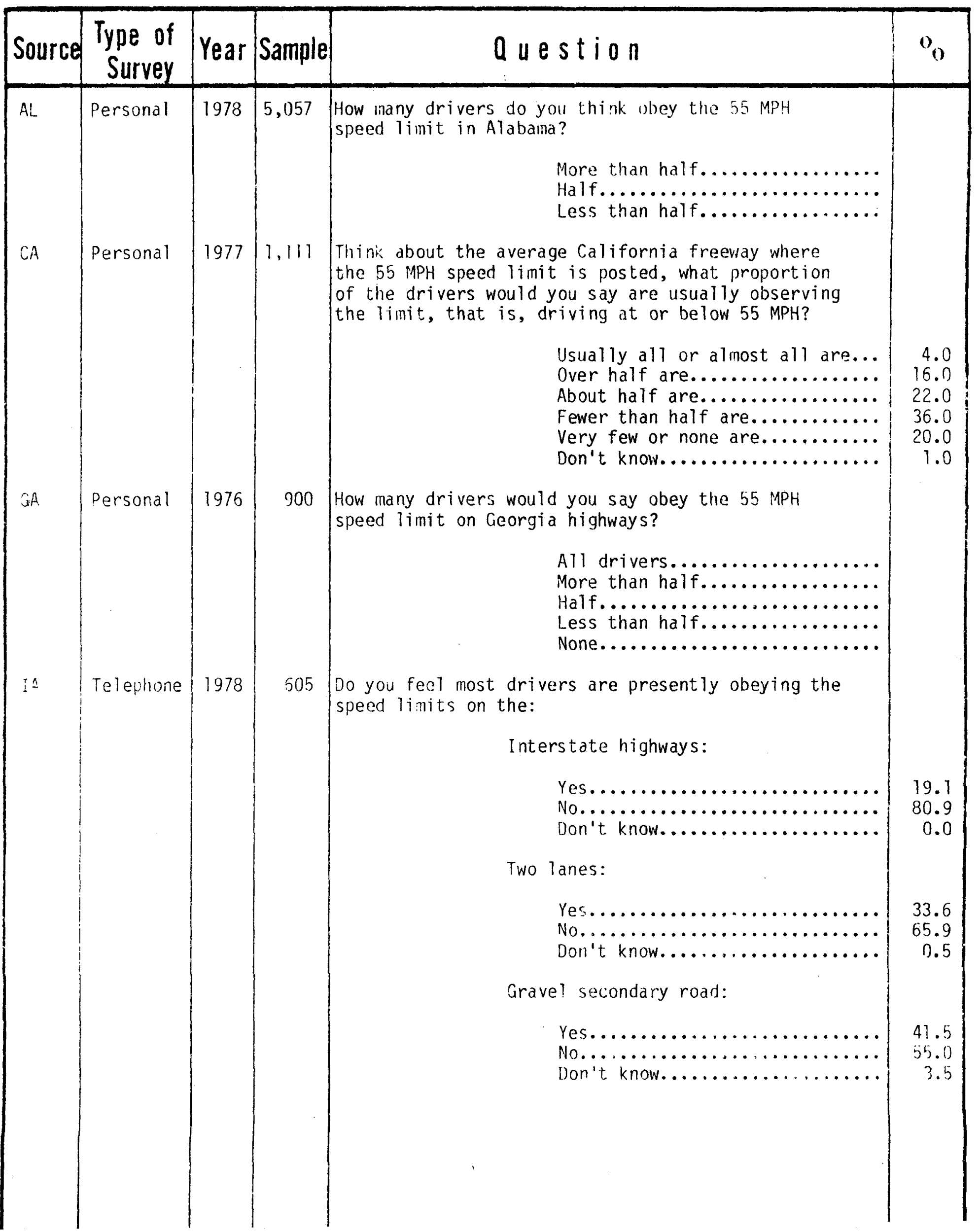




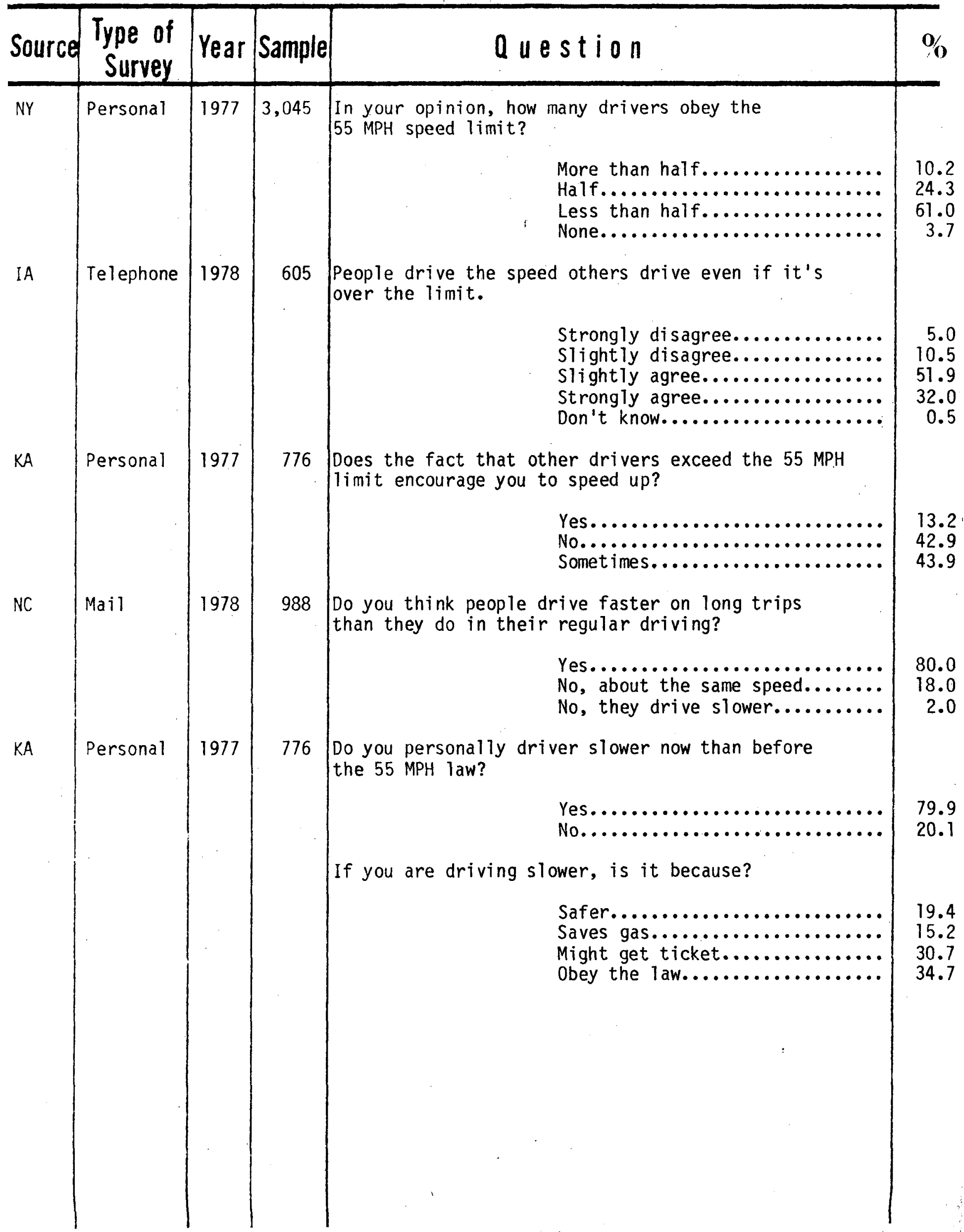




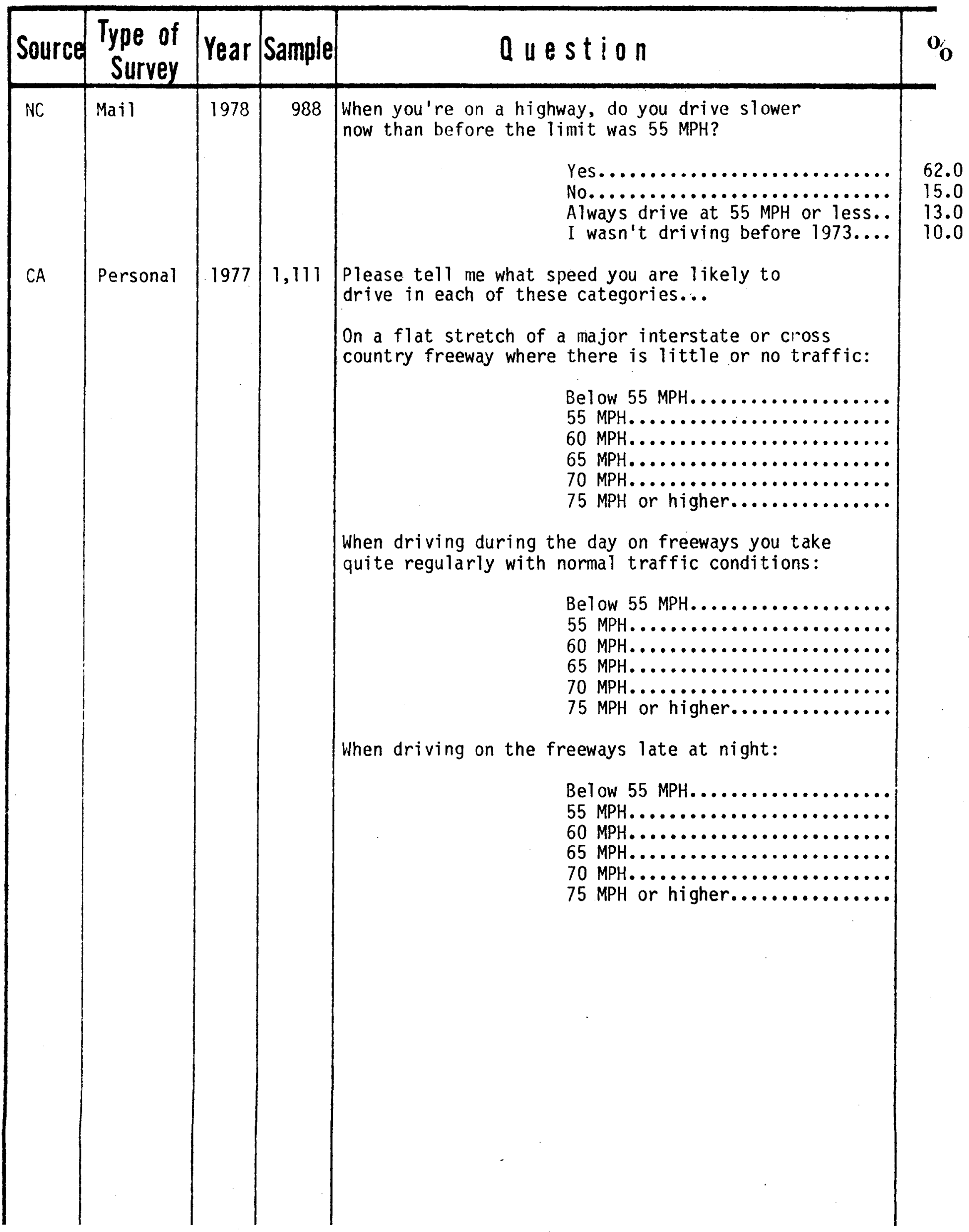




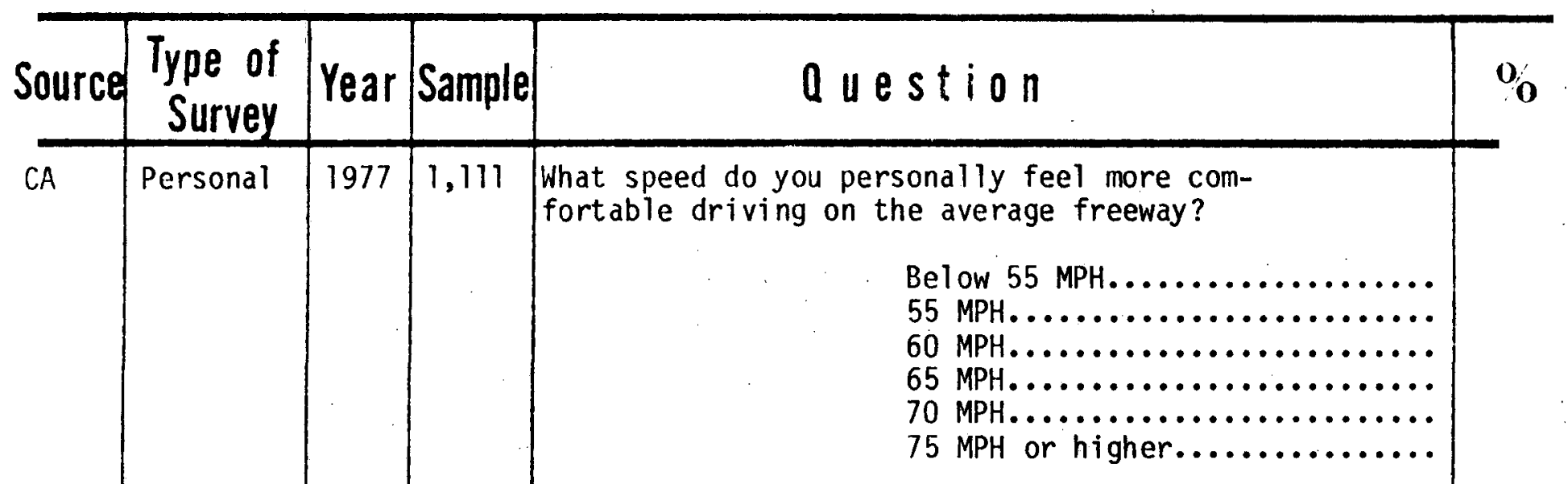

At what speed do you think you get your best mileage on the freeway?

Below $55 \mathrm{MPH}$

$55 \mathrm{MPH}$.

$60 \mathrm{MPH}$

$65 \mathrm{MPH}$

$70 \mathrm{MPH}$.

$75 \mathrm{MPH}$ or higher

At what speed on the average freeway do you feel you are going too fast to feel safe?

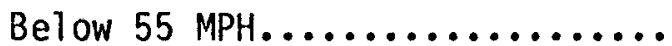
$55 \mathrm{MPH}$.

$60 \mathrm{MPH}$

$65 \mathrm{MPH}$

$70 \mathrm{MPH} . \ldots \ldots \ldots \ldots \ldots \ldots \ldots \ldots \ldots$

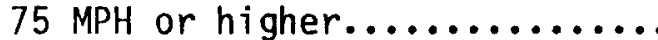




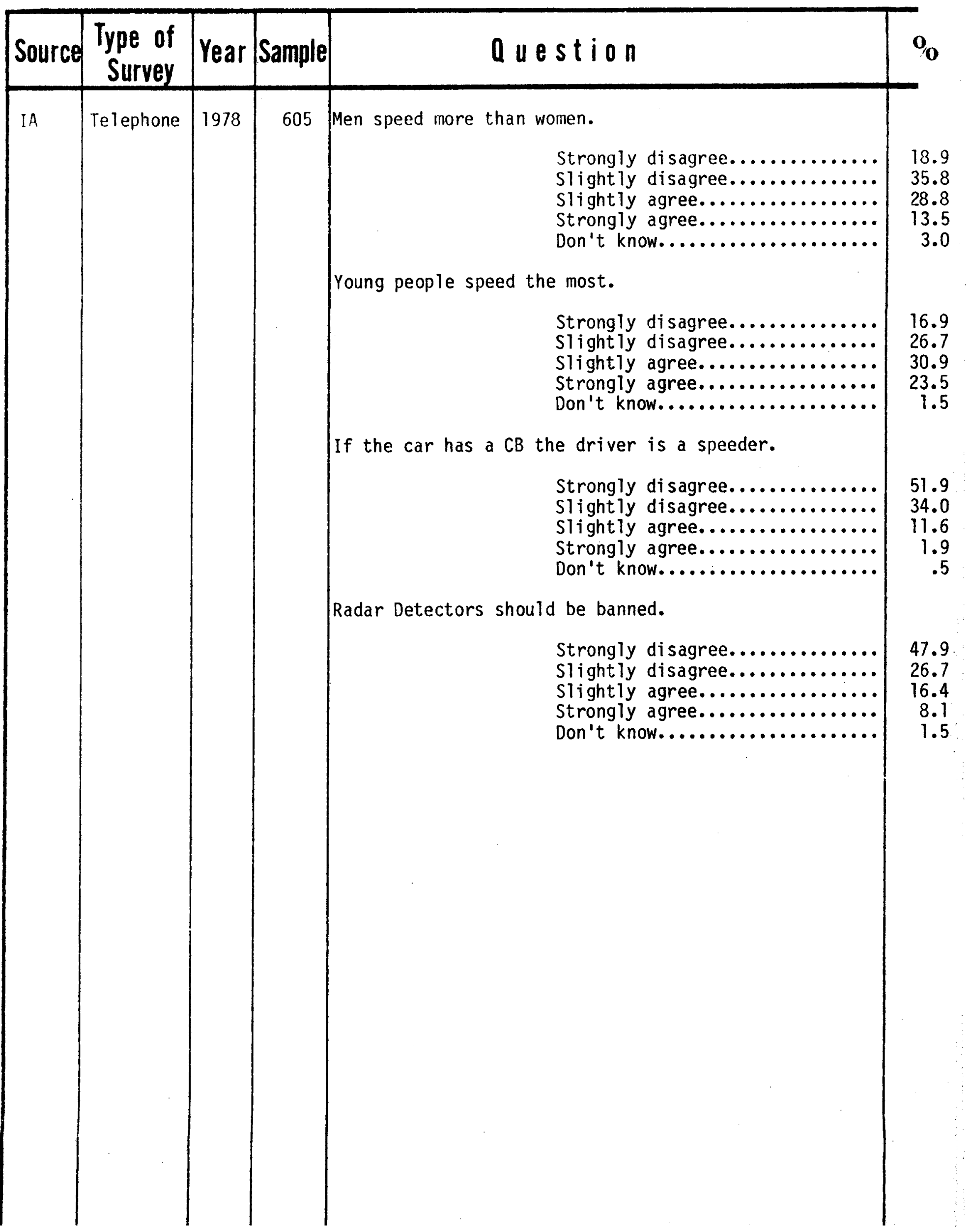


KNOWLEDGE $\longrightarrow$ 


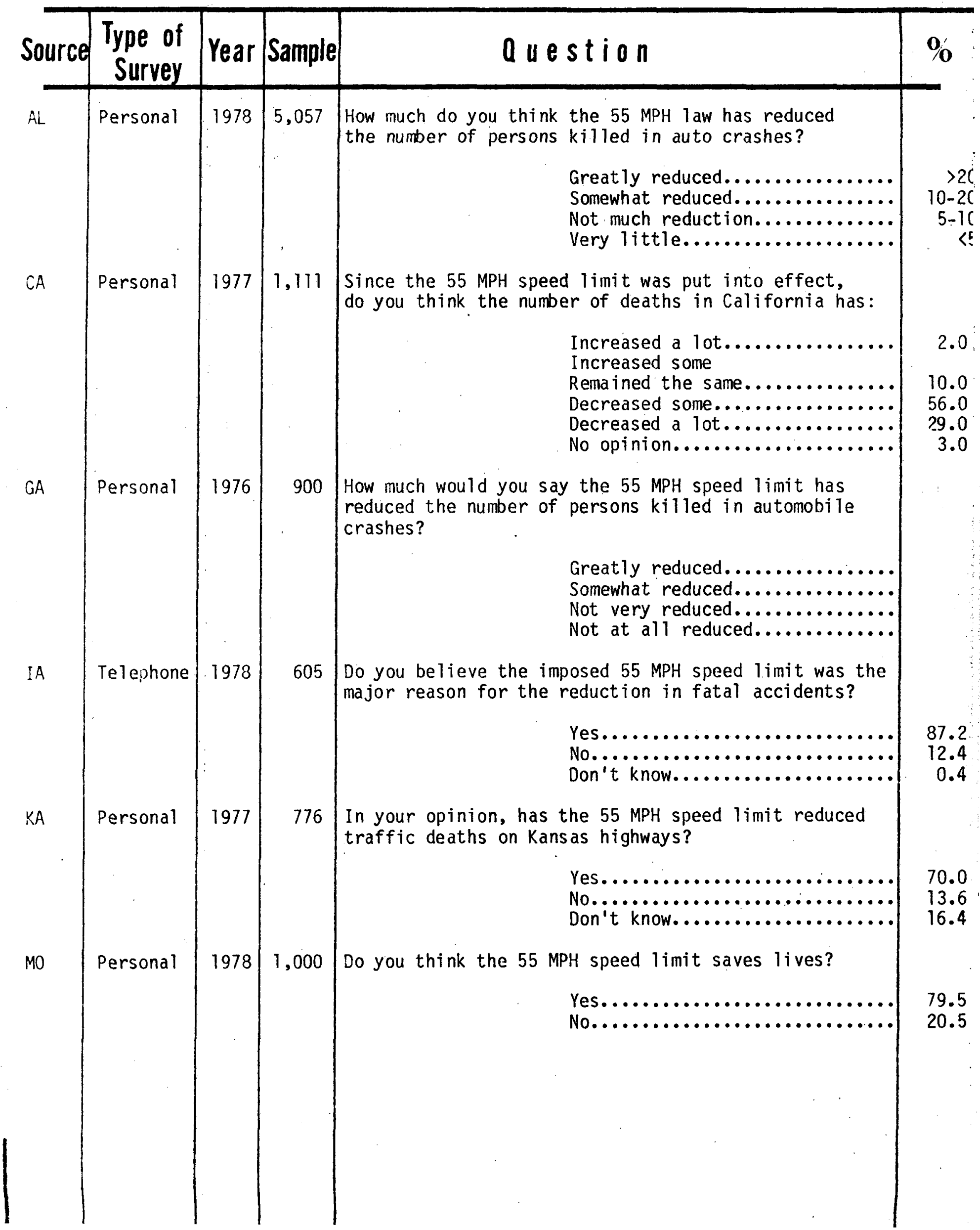




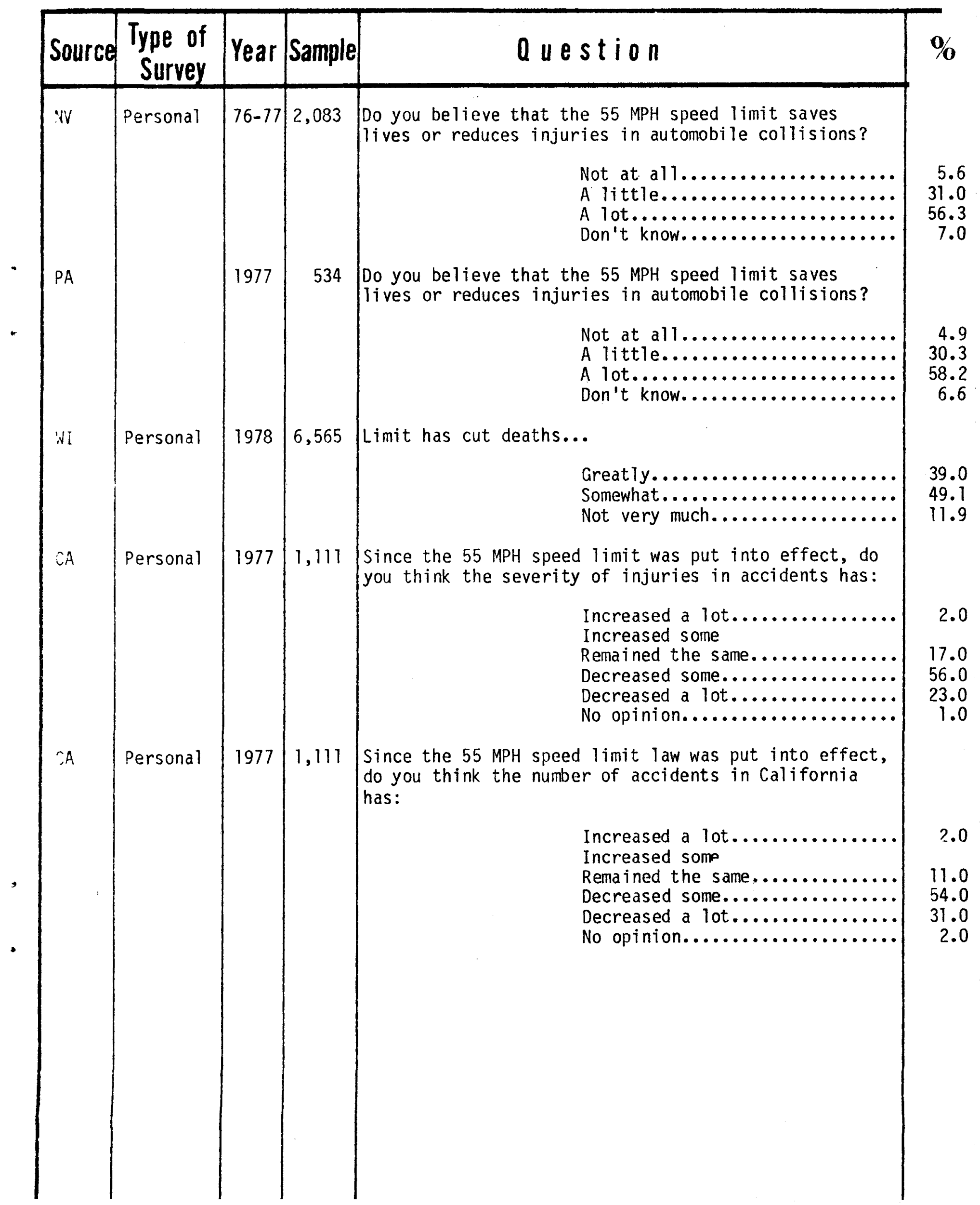




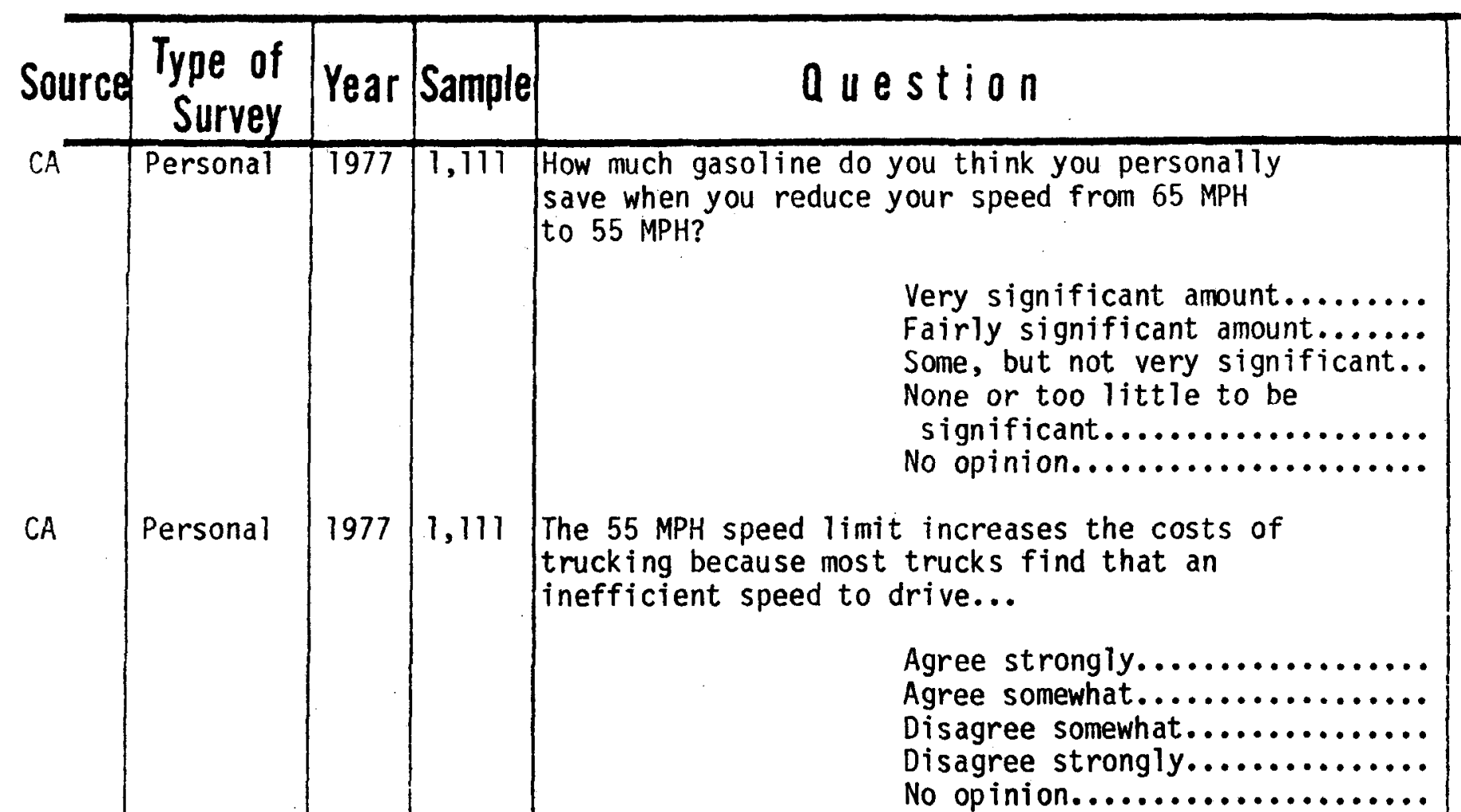

$\%$ 
$\left(\begin{array}{ccc}\text { ENFORCEMENT } & \text { OF } \\ 55 & 1 & 1 \\ \text { SPEED } & \text { LIMIT }\end{array}\right)$ 


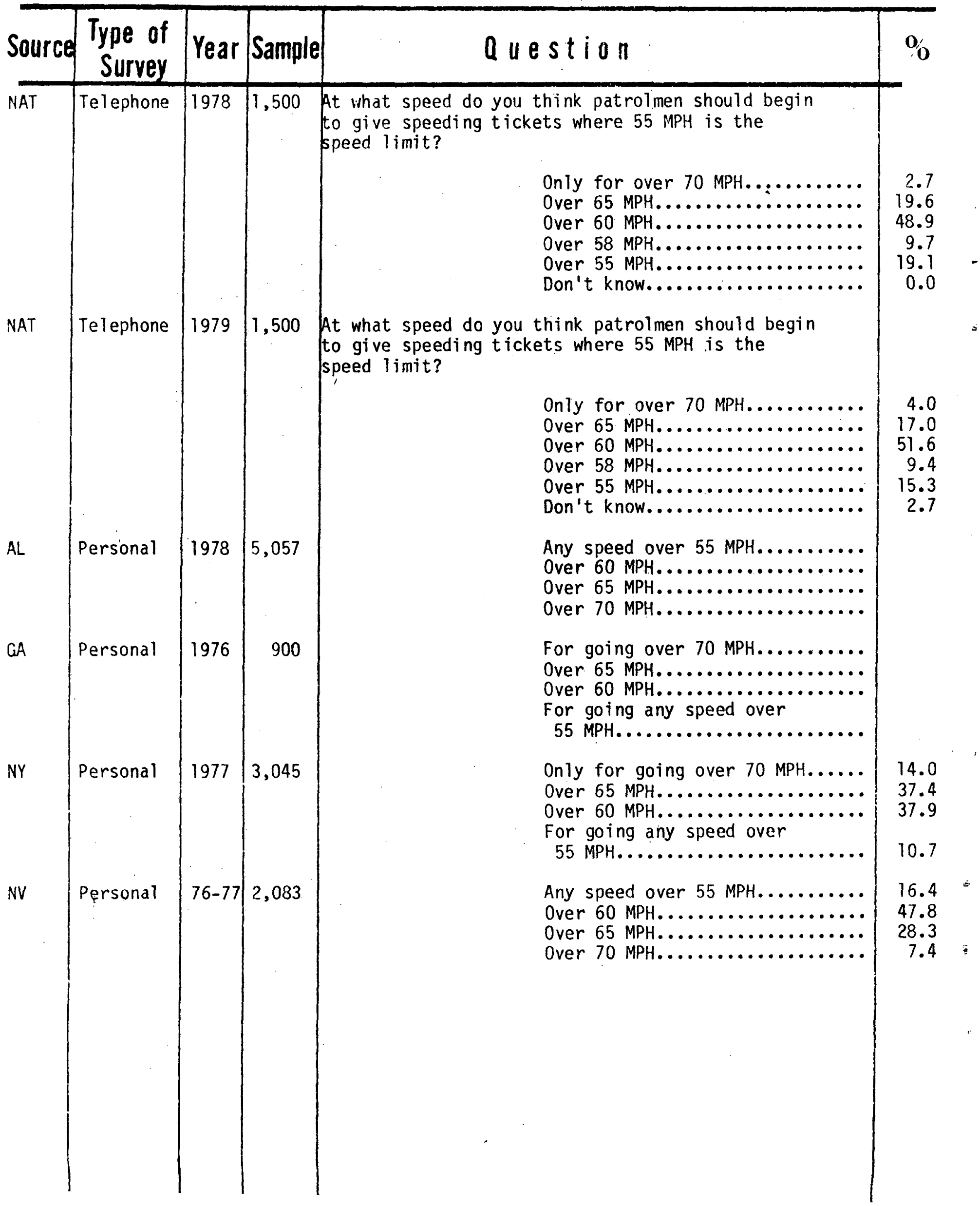




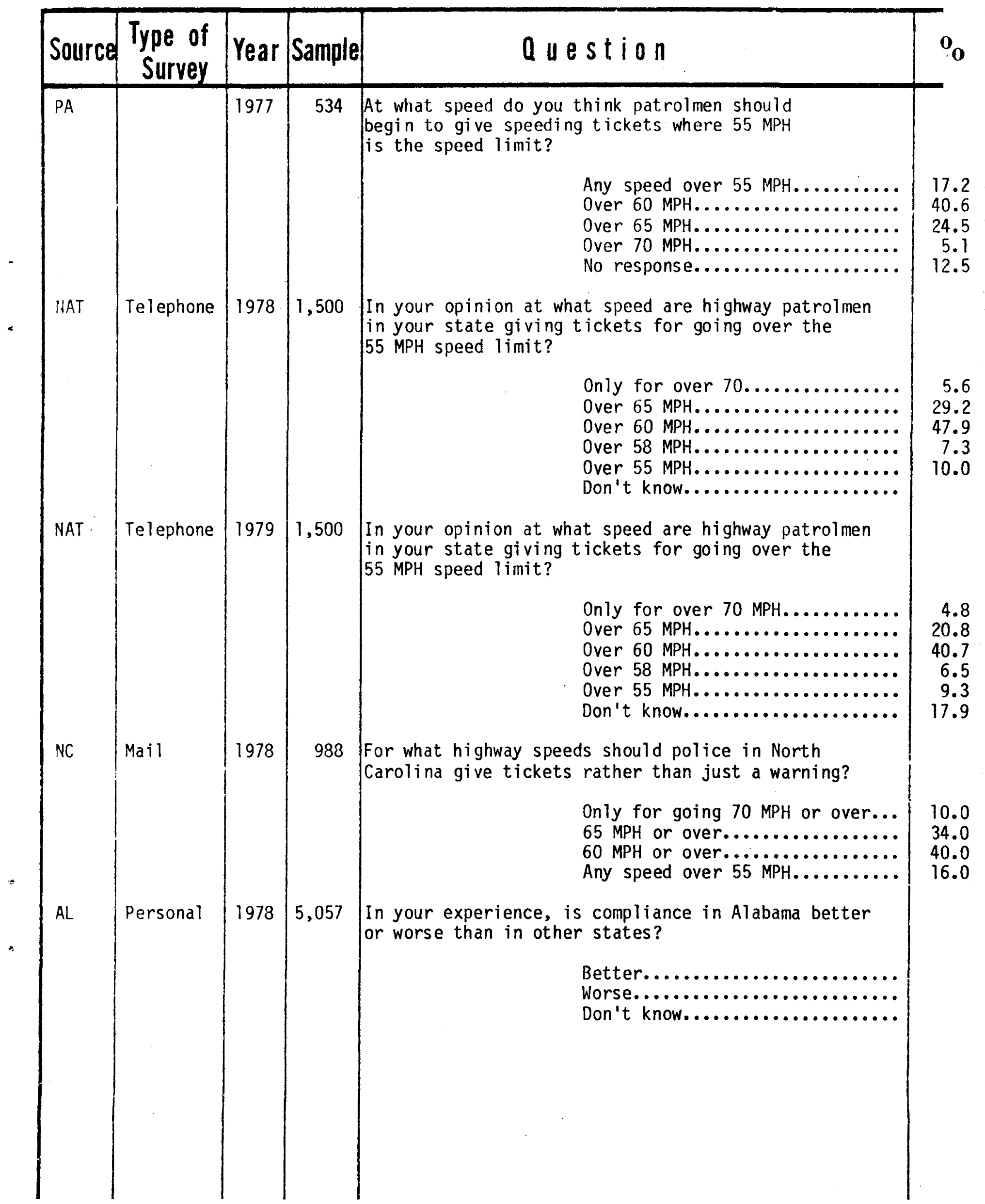




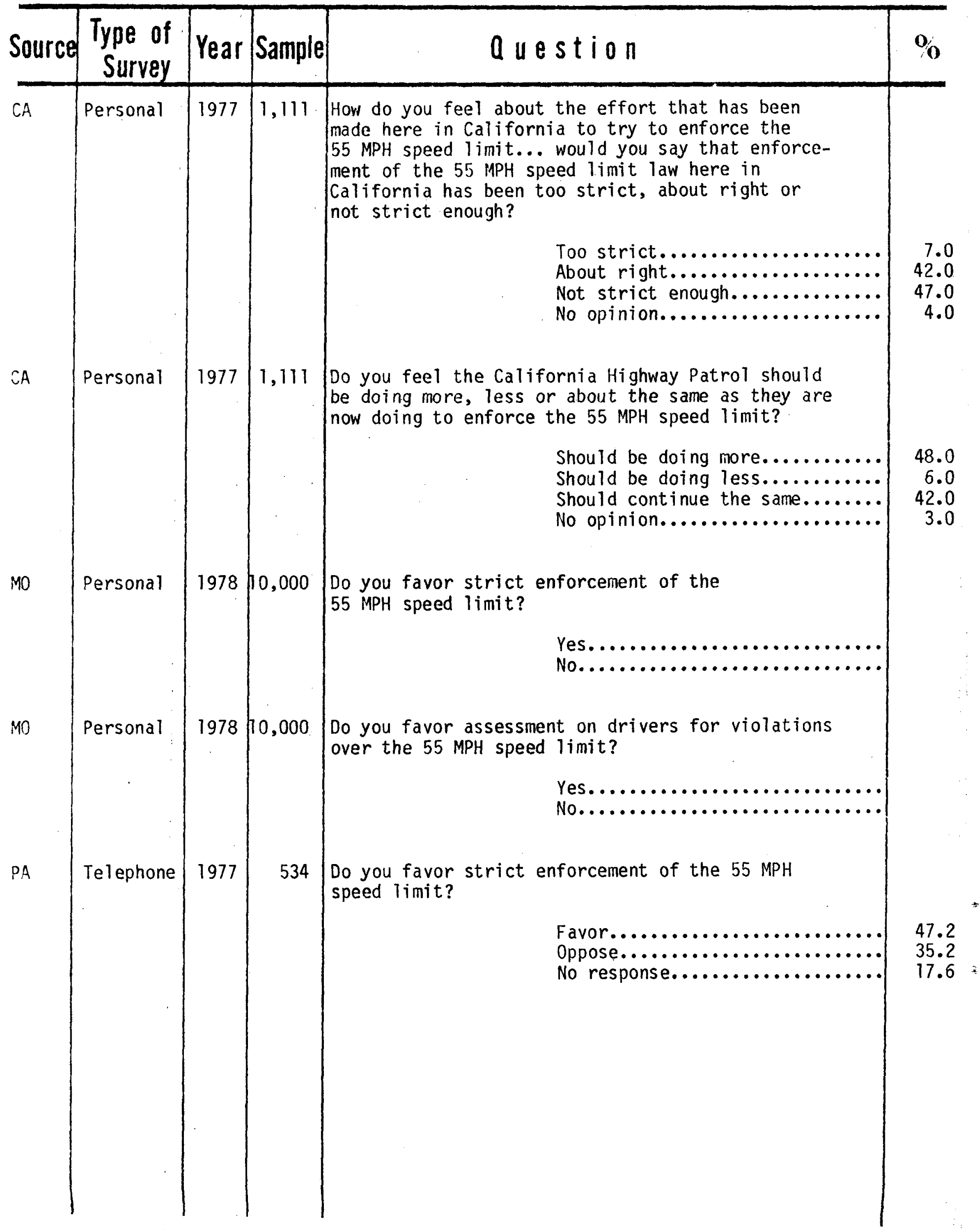




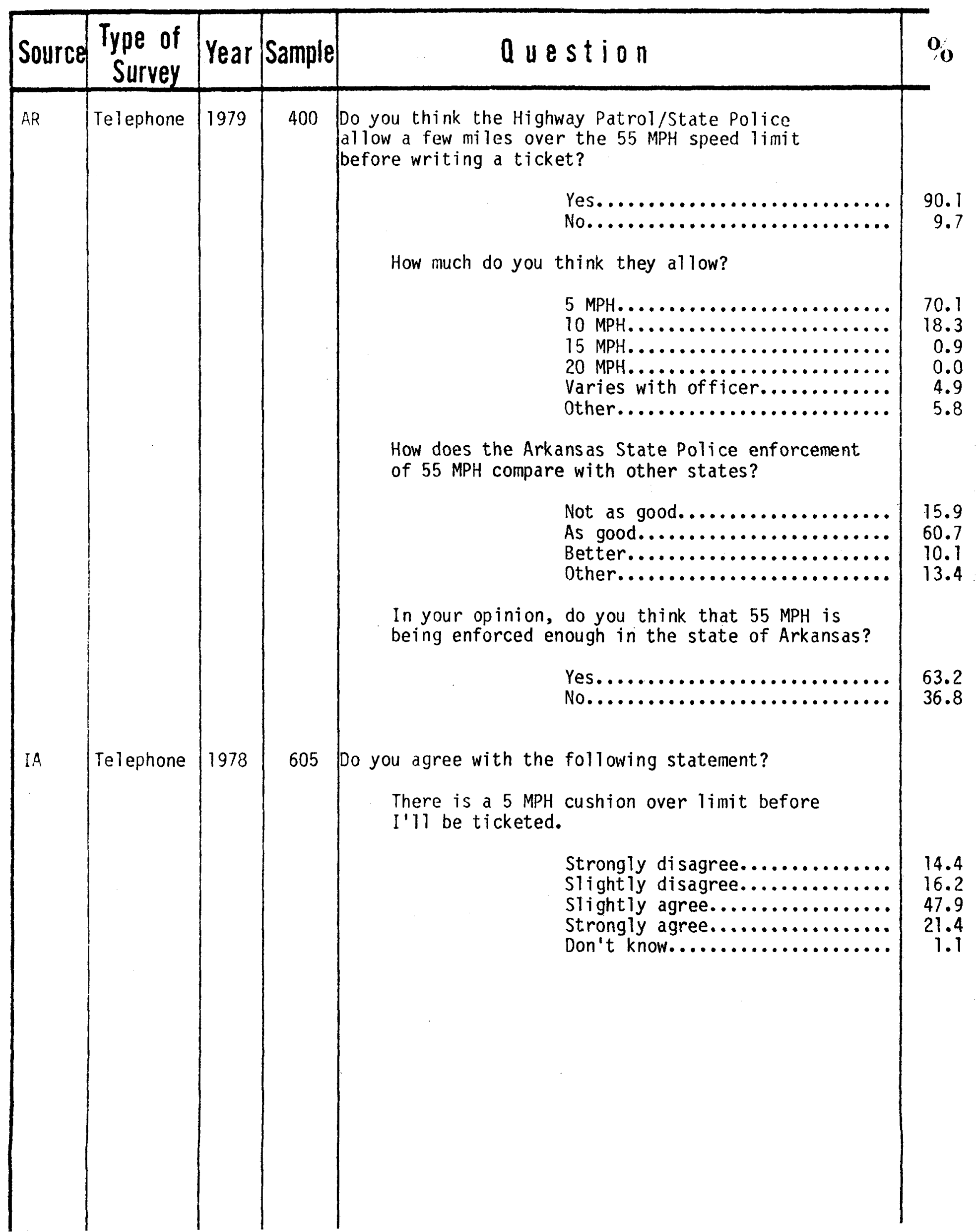




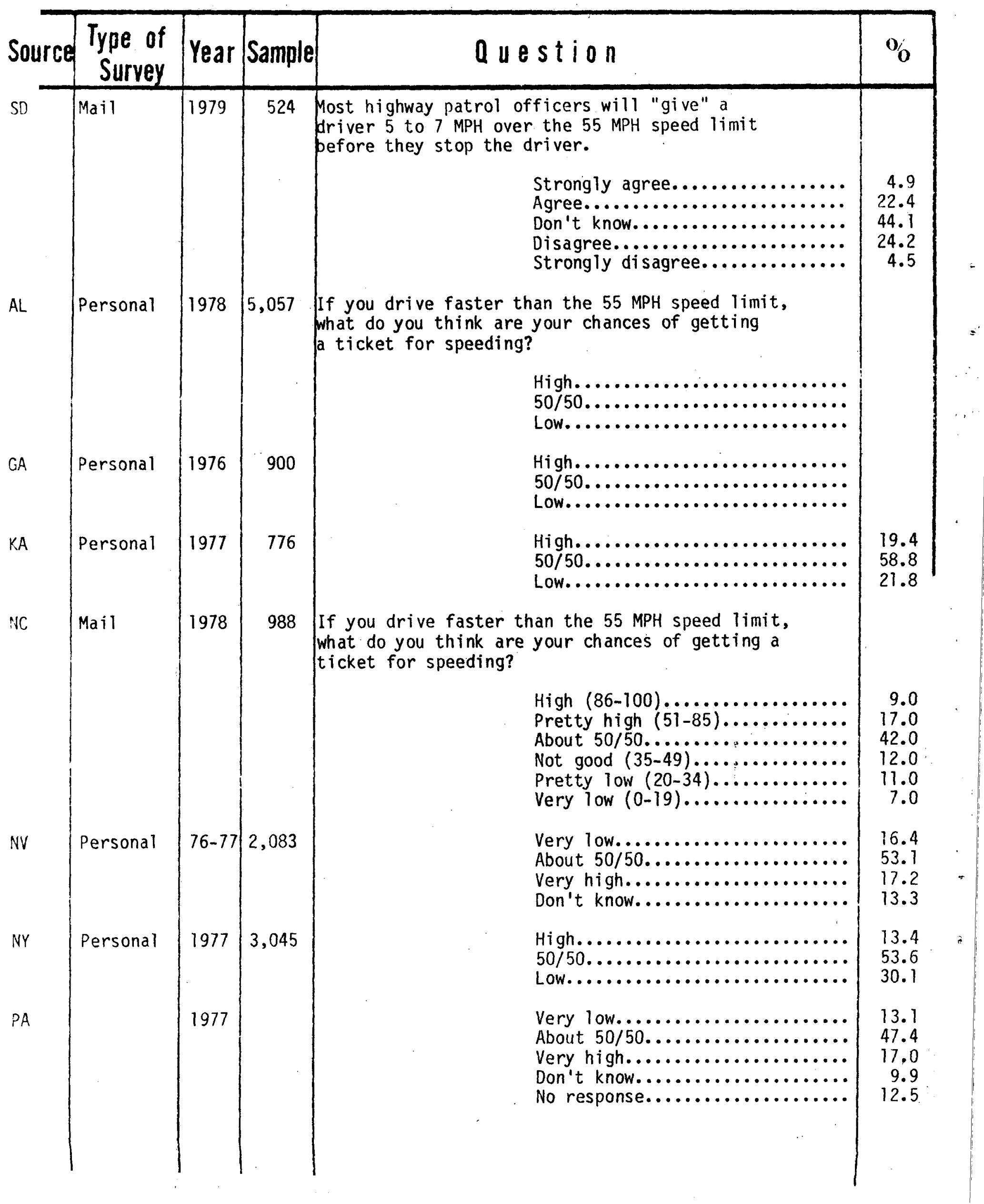




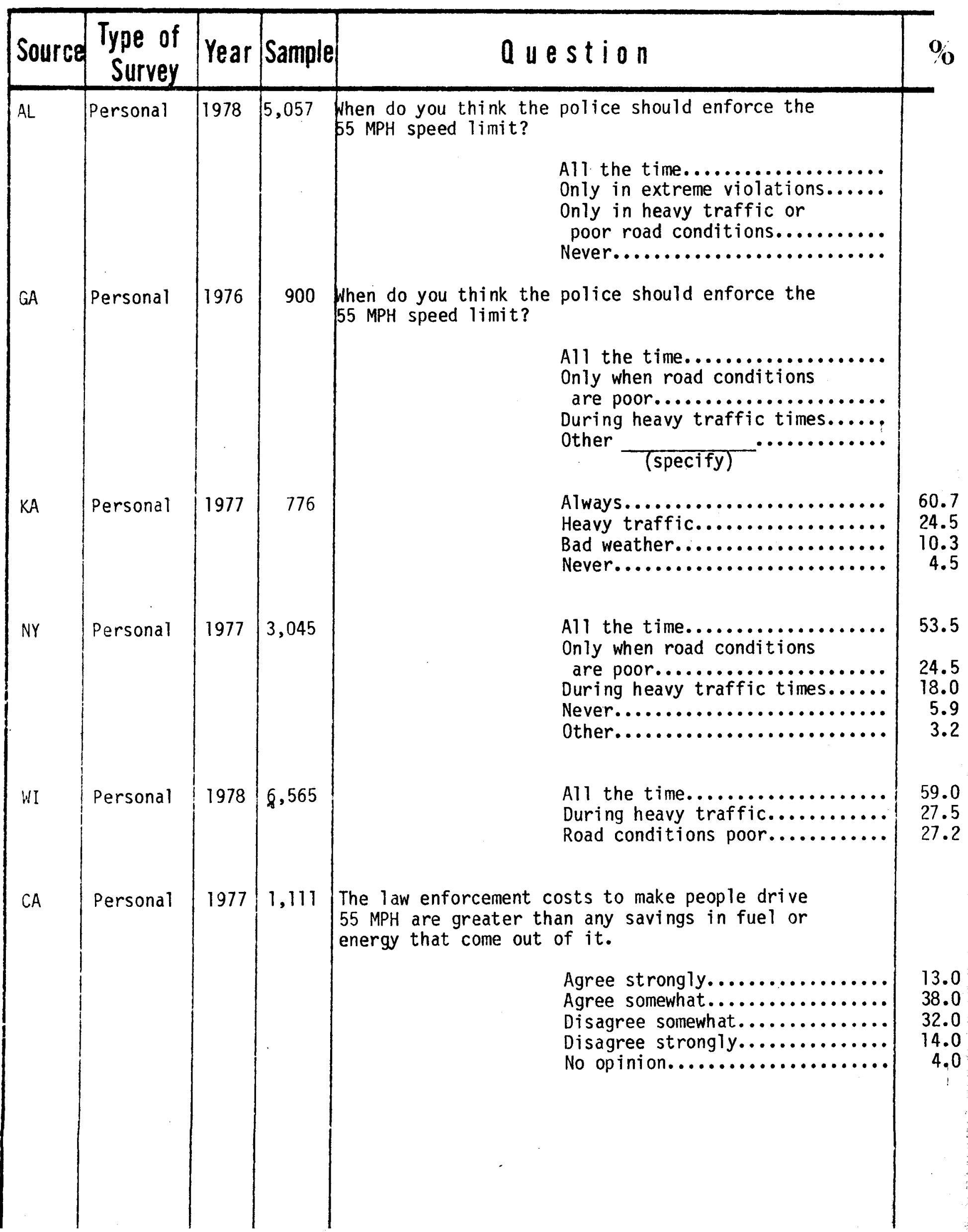




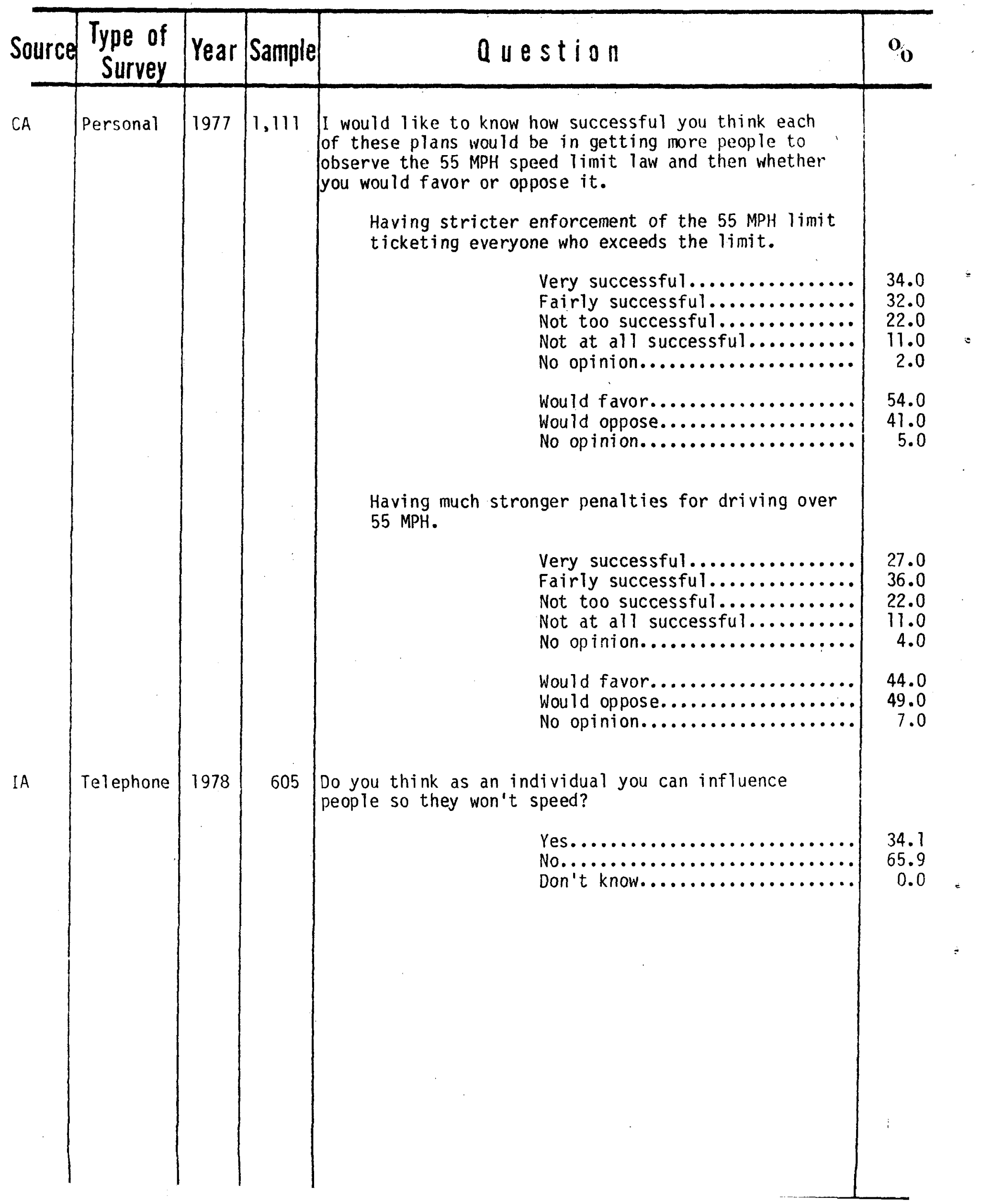




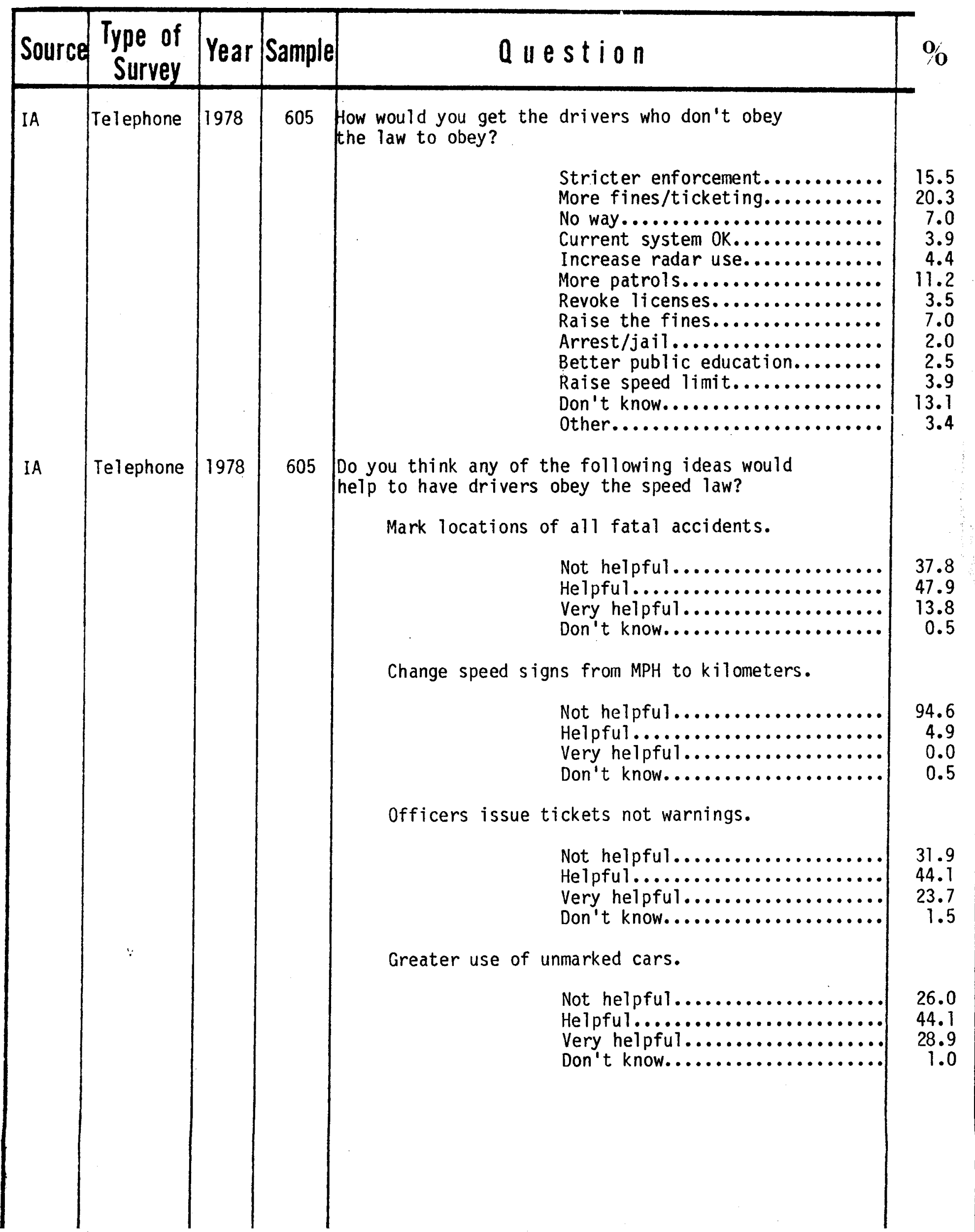




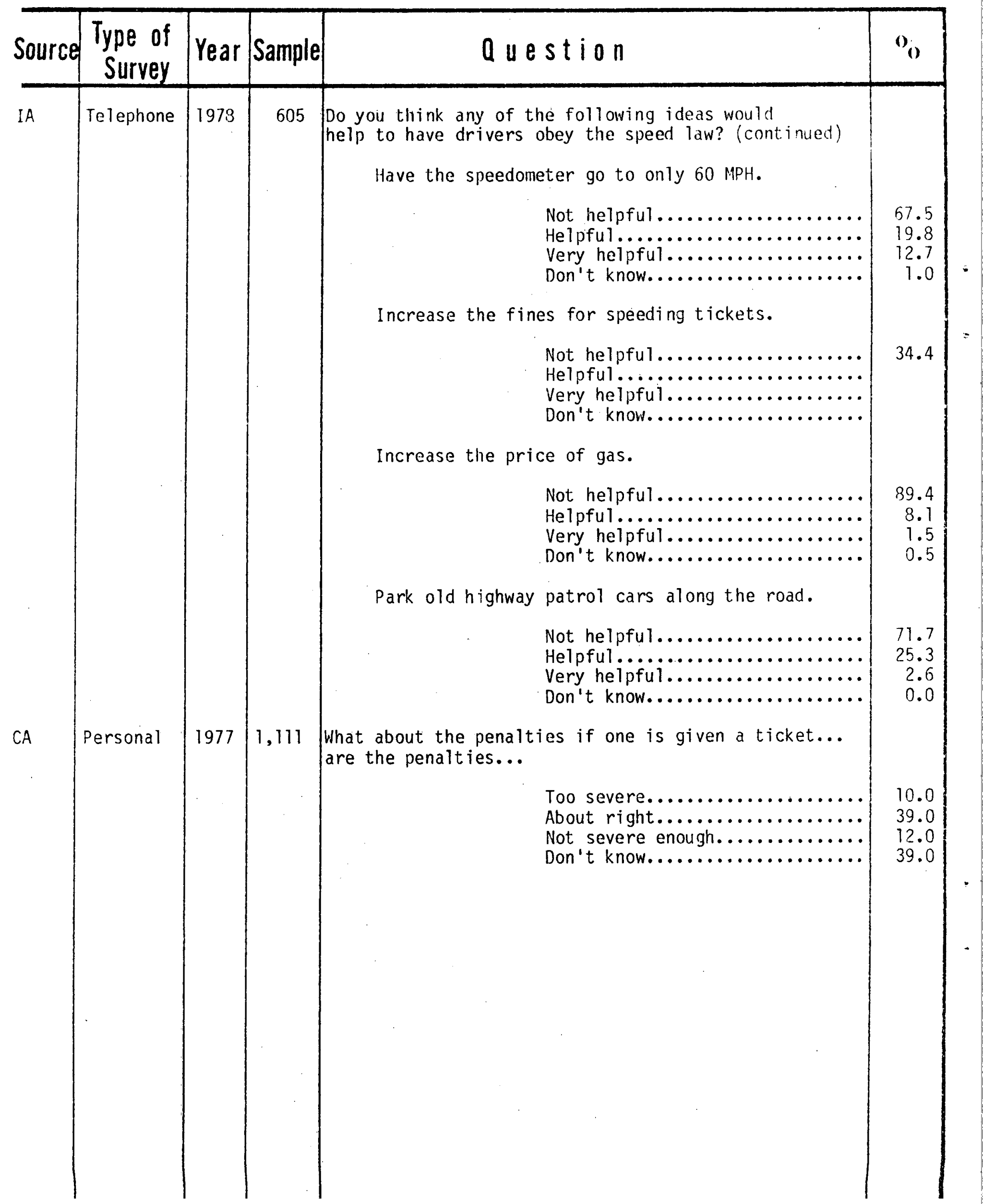




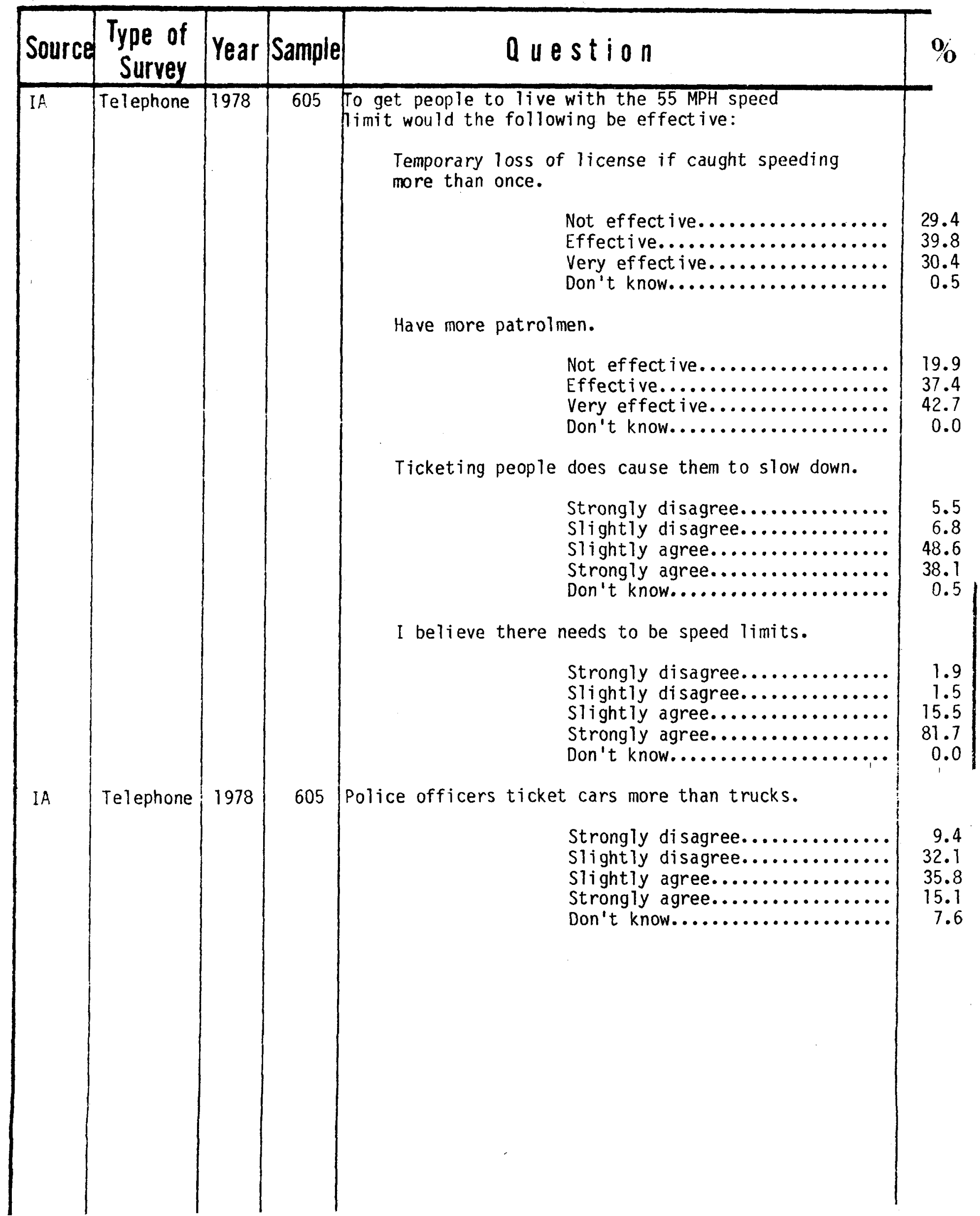




\begin{tabular}{|c|c|c|c|c|}
\hline Source & $\begin{array}{l}\text { Type of } \\
\text { Survey }\end{array}$ & Year & Sample & Question \\
\hline SD & Mail & 1979 & 524 & 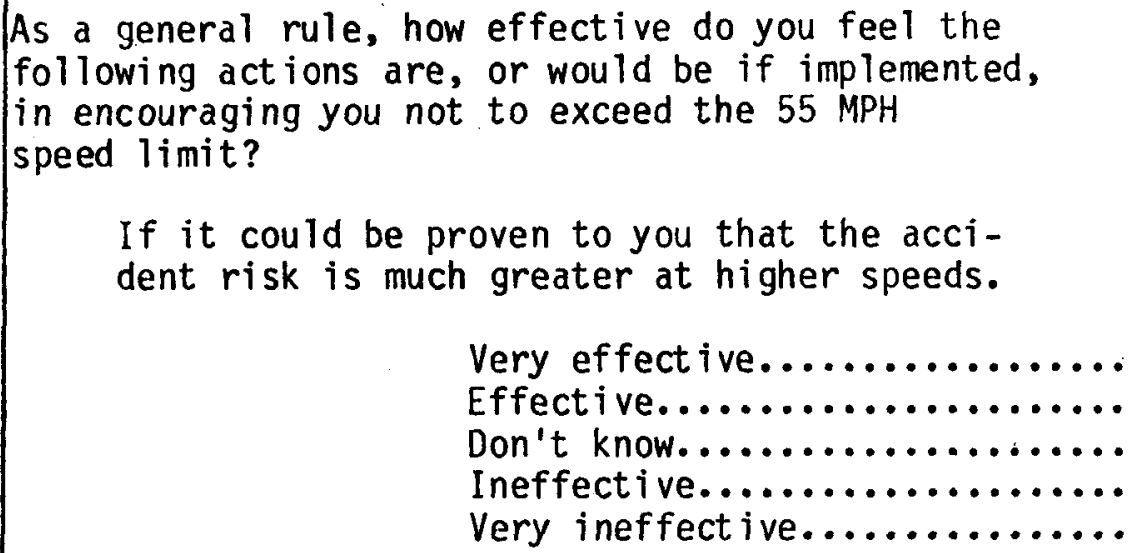 \\
\hline
\end{tabular}

If it could be proven to you that there was a chance you would be arrested for speeding.

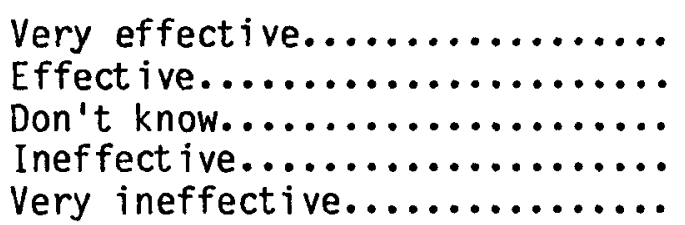

If it could be proven to you that you got much better gas mileage at speeds of $55 \mathrm{MPH}$ or slower.

Very effective............... Effective..................

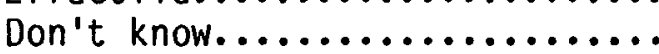
Ineffective................. Very ineffect ive.............

30.8

46.2

11.9

9.7

1.4

If you knew for sure that there would be a severe penalty if you were caught exceeding the $55 \mathrm{MPH}$ limit.

Very effective..............

Effective..................

Don't know.

Ineffective.

Very ineffect ive 0.0 .0 .0 .0 .0$.

If each vehicle were equipped with a mandatory device (such as a buzzer) which informed you when you exceeded $55 \mathrm{MPH}$.

Very effective...............

Effective..................

Don't know...................

Ineffective.................

Very ineffect ive

21.6

48.3

11.5

17.0

1.6

38.5

44.7

10.9

5.1

1.4

16.4

36.8

12.9

25.8

8.1 


\begin{tabular}{|c|c|c|c|c|c|}
\hline Source & $\left\{\begin{array}{l}\text { Type of } \\
\text { Survey }\end{array}\right.$ & Year & Sample & Question & $o_{0}$ \\
\hline SD & Mail & 1979 & 524 & 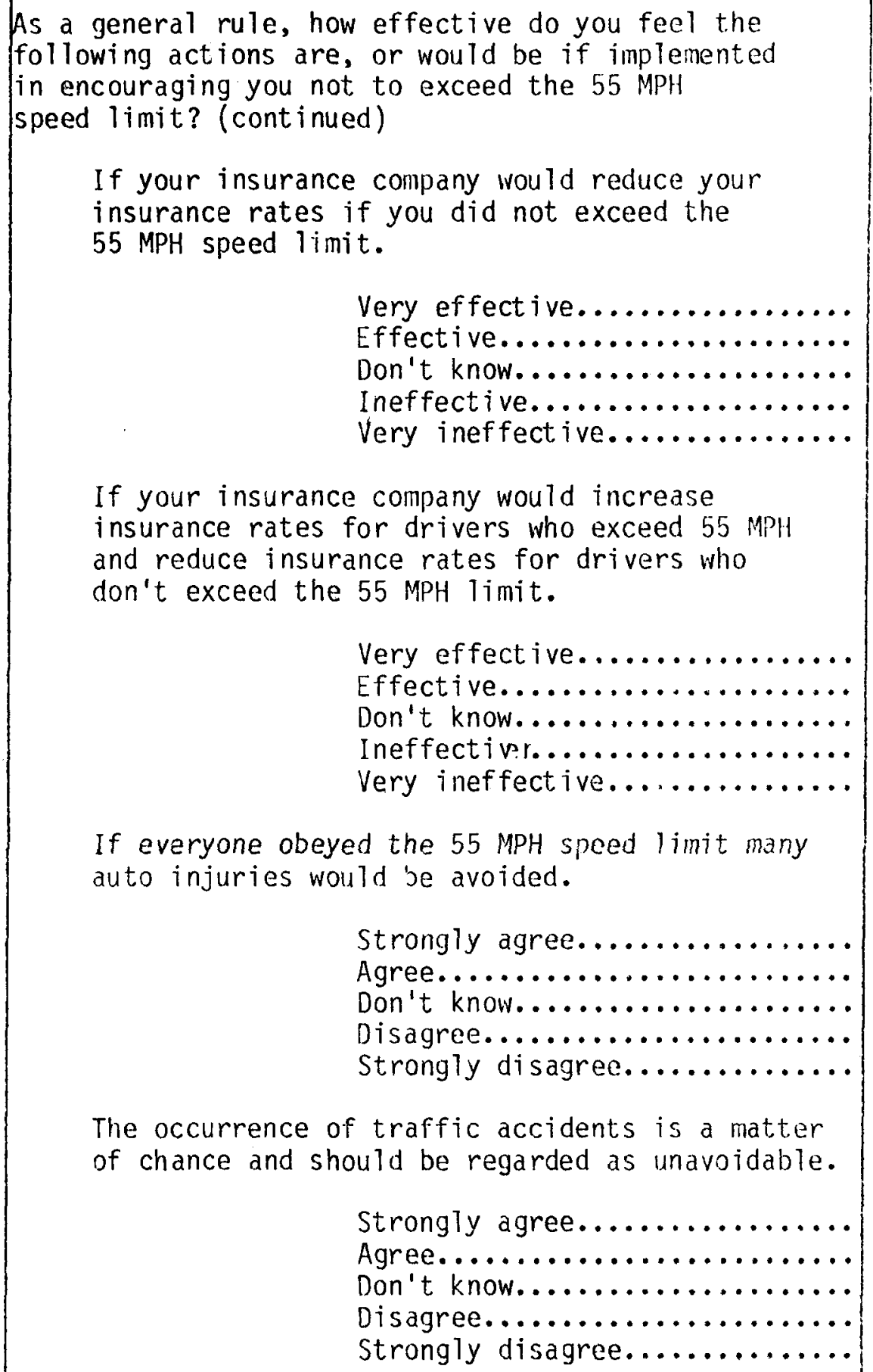 & $\begin{array}{r} \\
29.0 \\
38.0 \\
16.3 \\
14.4 \\
2.3 \\
\\
\\
21.8 \\
49.9 \\
9.5 \\
16.4 \\
2.3 \\
\\
\\
1.4 \\
9.2 \\
10.2 \\
53.6 \\
25.6\end{array}$ \\
\hline
\end{tabular}




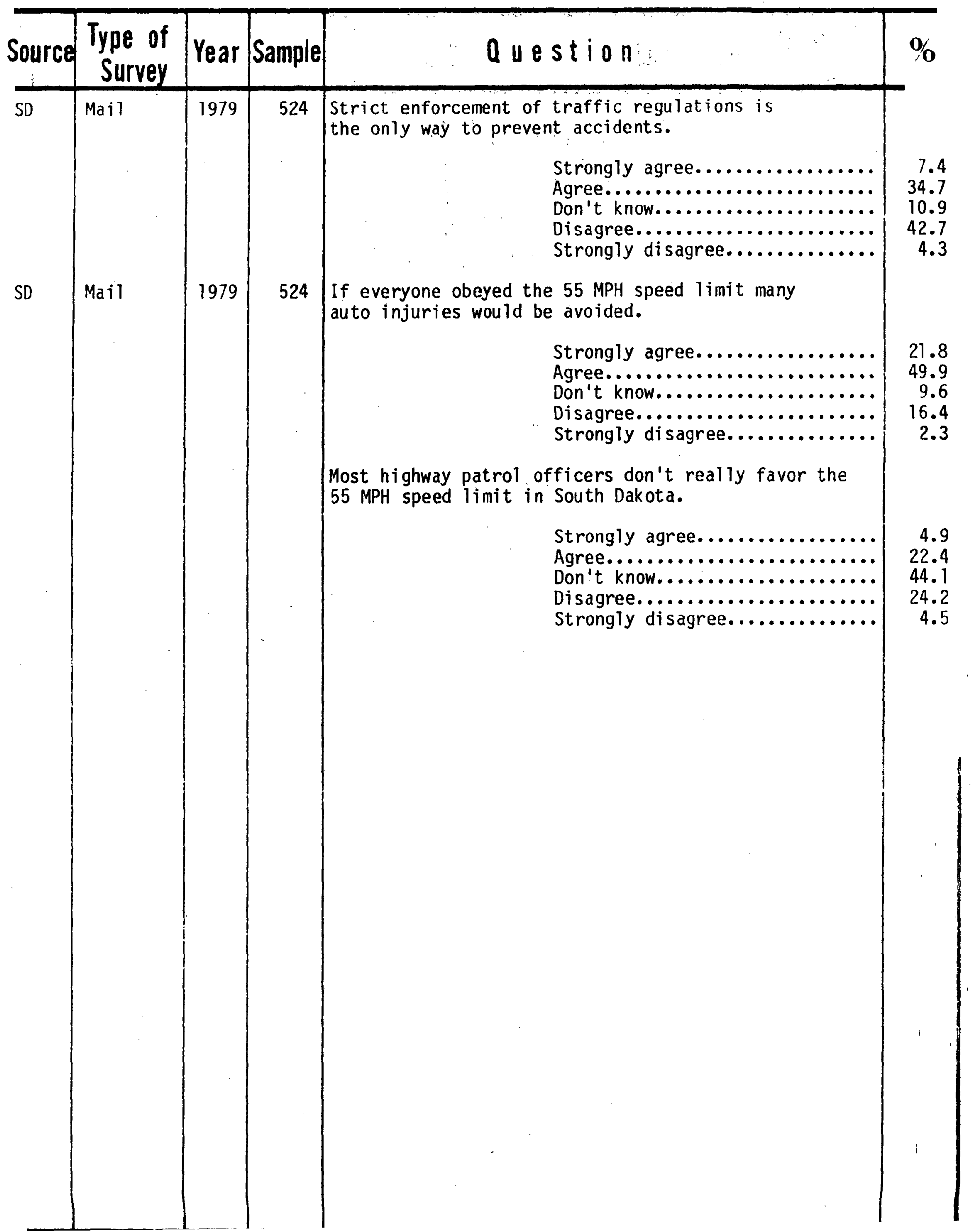


BEHAVIOR 


\begin{tabular}{|c|c|c|c|c|c|}
\hline Source & $\begin{array}{c}\text { Type of } \\
\text { Survey }\end{array}$ & Year & Sample & Question & o \\
\hline IA & Tel ephone & 1978 & 605 & 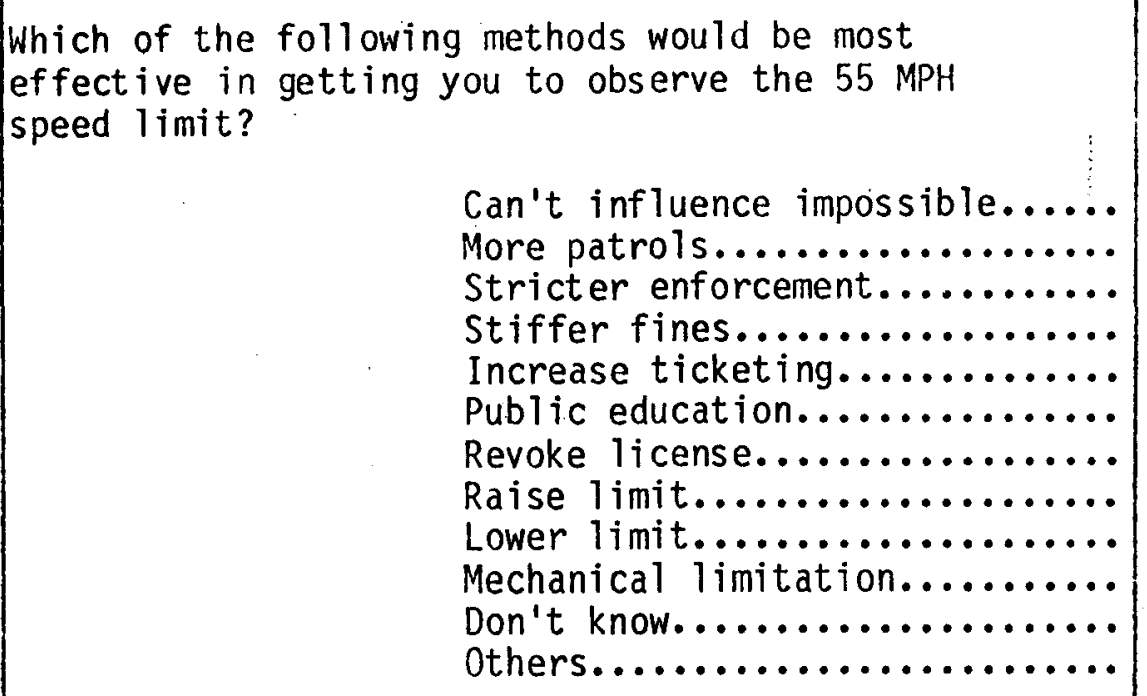 & $\begin{array}{r}20.9 \\
9.5 \\
11.6 \\
8.0 \\
9.0 \\
12.1 \\
2.9 \\
3.5 \\
1.0 \\
4.8 \\
11.7 \\
5.5\end{array}$ \\
\hline NC & Mail & 1978 & 988 & 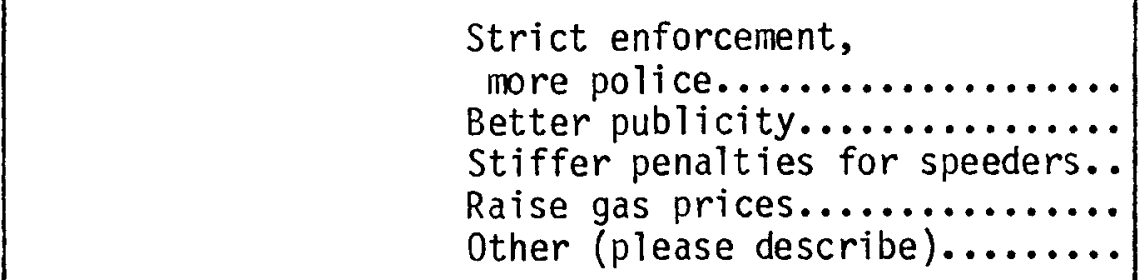 & $\begin{array}{l}23.4 \\
18.2 \\
20.2 \\
20.8 \\
17.4\end{array}$ \\
\hline NV & Personal & $76-77$ & 2,083 & 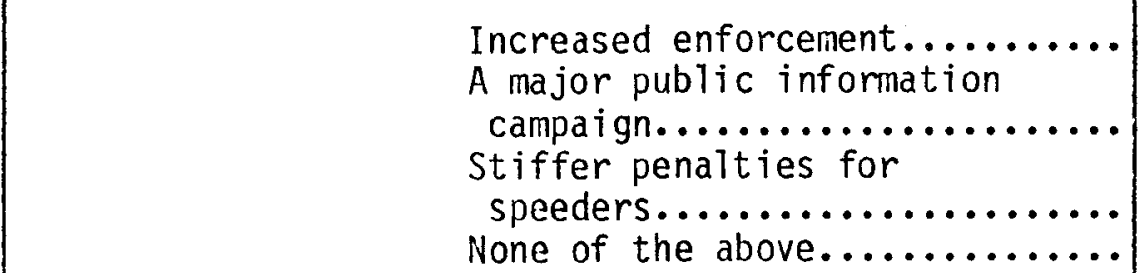 & $\begin{array}{r}40.0 \\
17.0 \\
35.0 \\
1.0\end{array}$ \\
\hline PA & & 1977 & 534 & 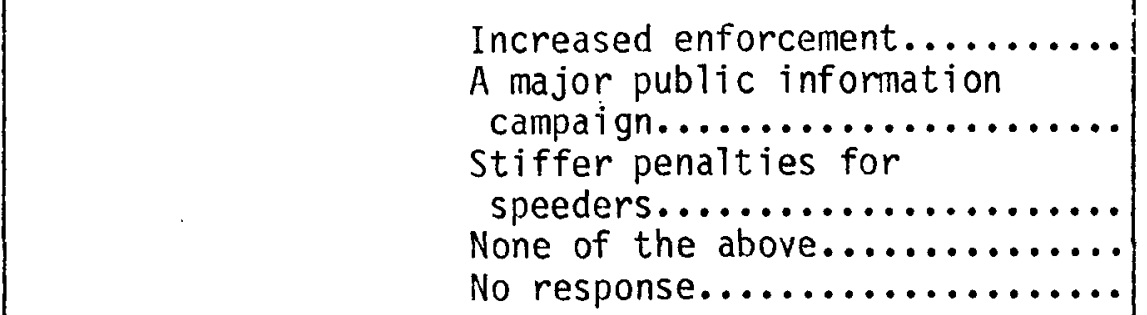 & $\begin{array}{l}23.4 \\
18.2 \\
20.2 \\
20.8 \\
17.4\end{array}$ \\
\hline PA & Telephone & 1977 & 500 & 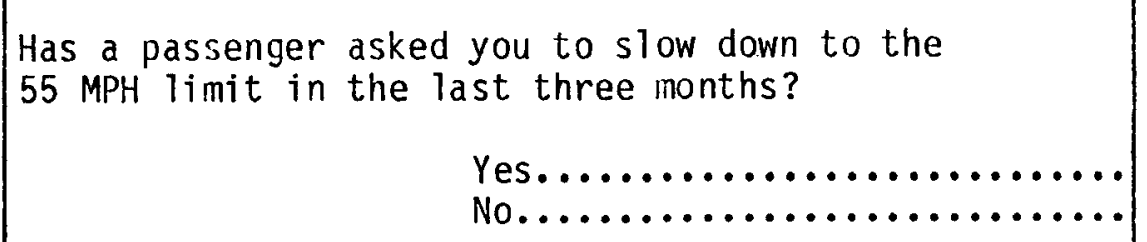 & $\begin{array}{r}5.0 \\
95.0\end{array}$ \\
\hline
\end{tabular}




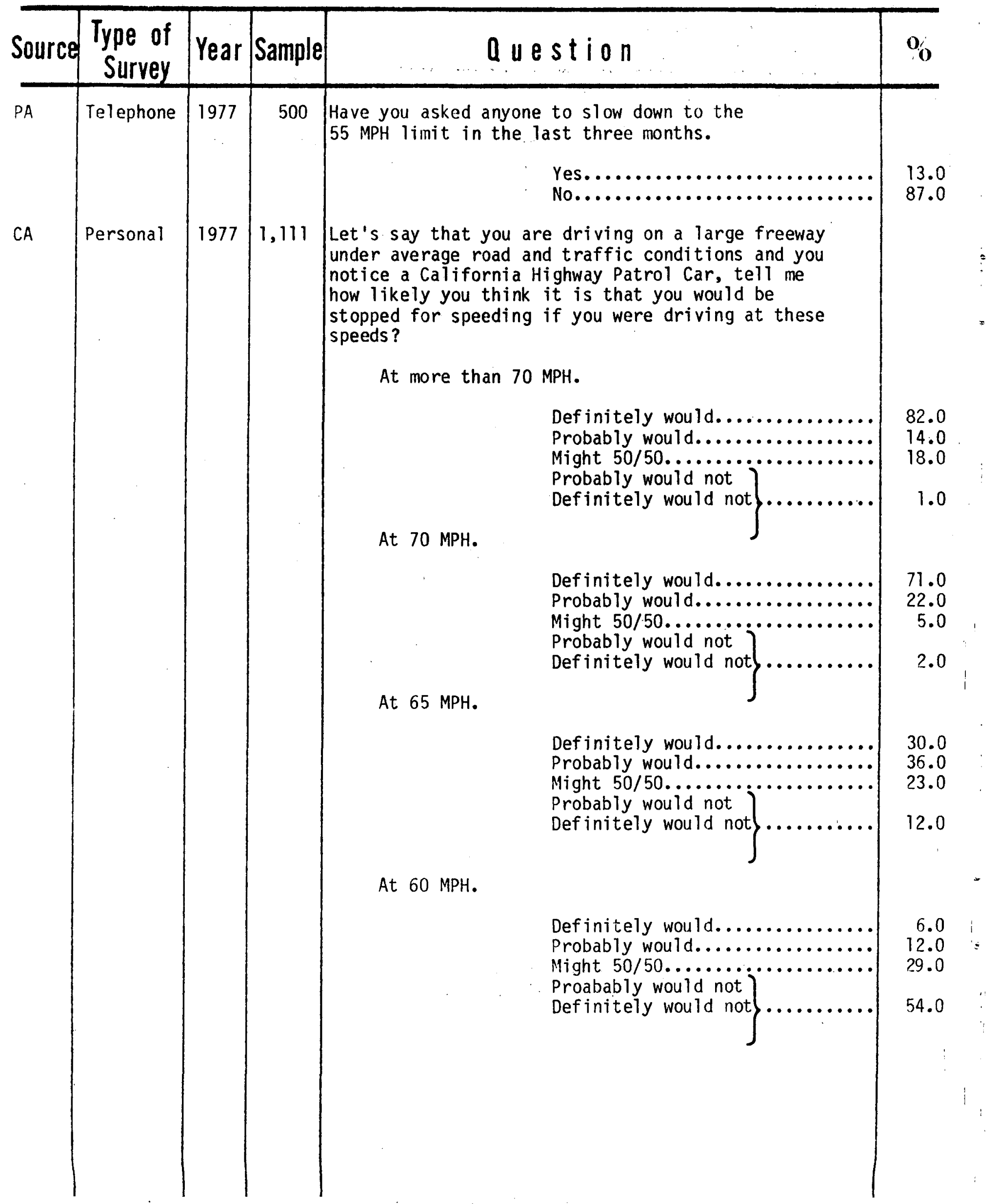




\section{KNOWLEDGE \\ KNOWLEDGE}




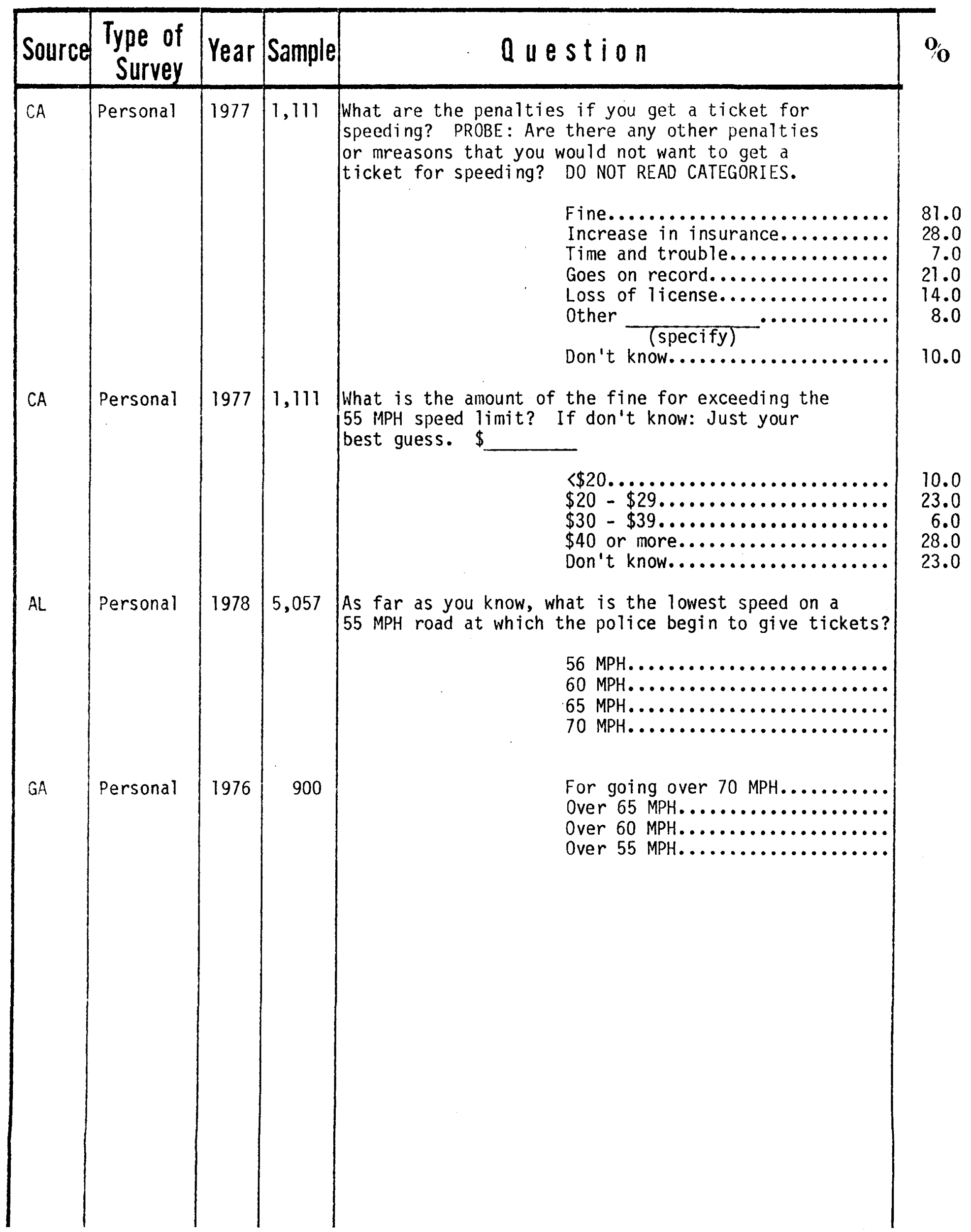




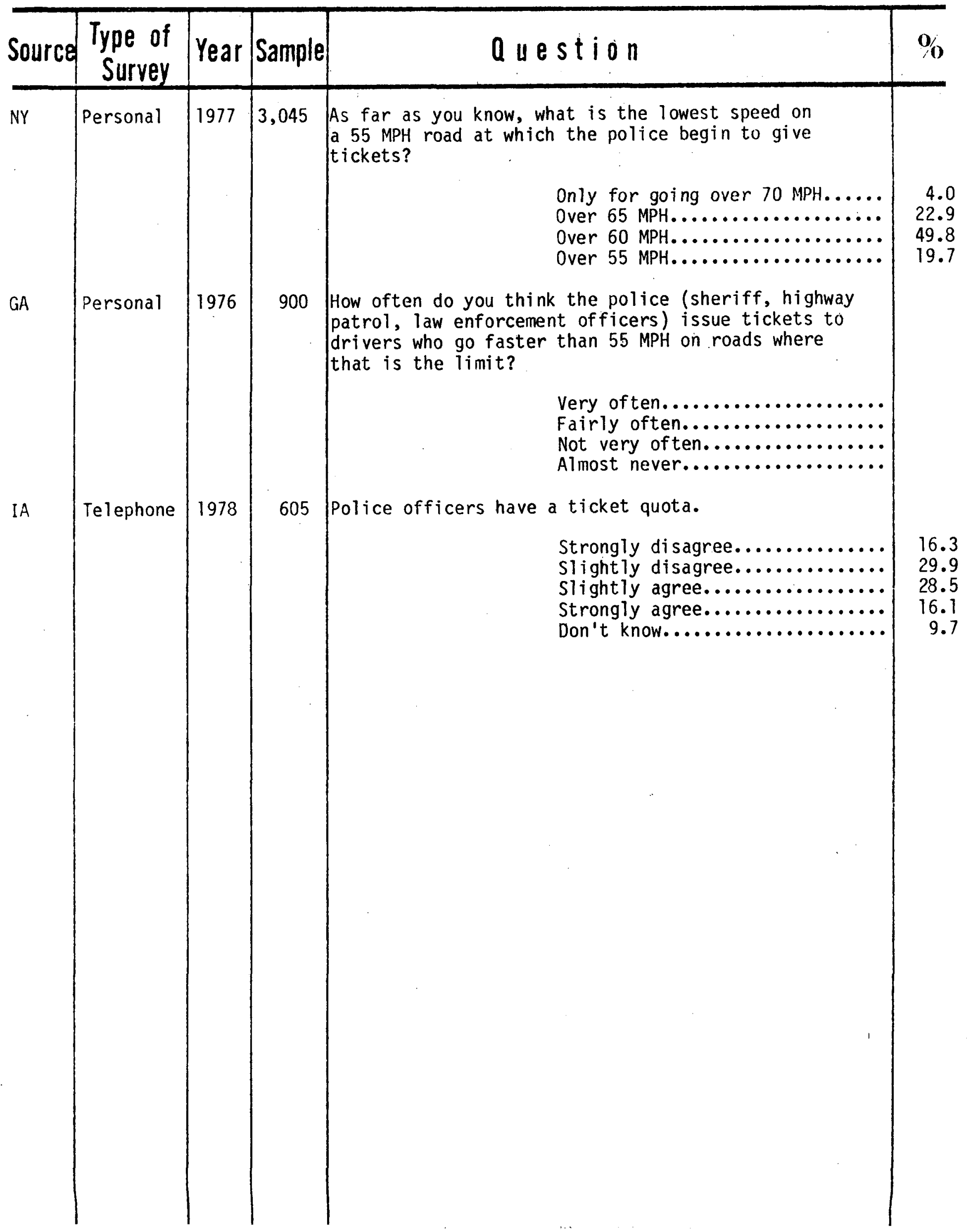




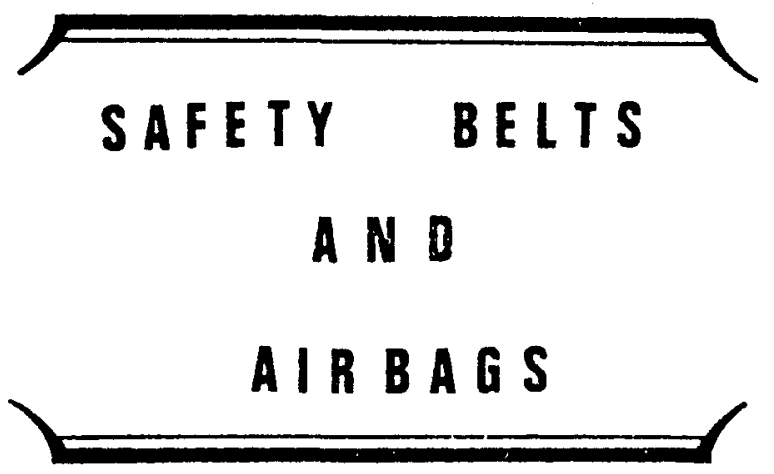




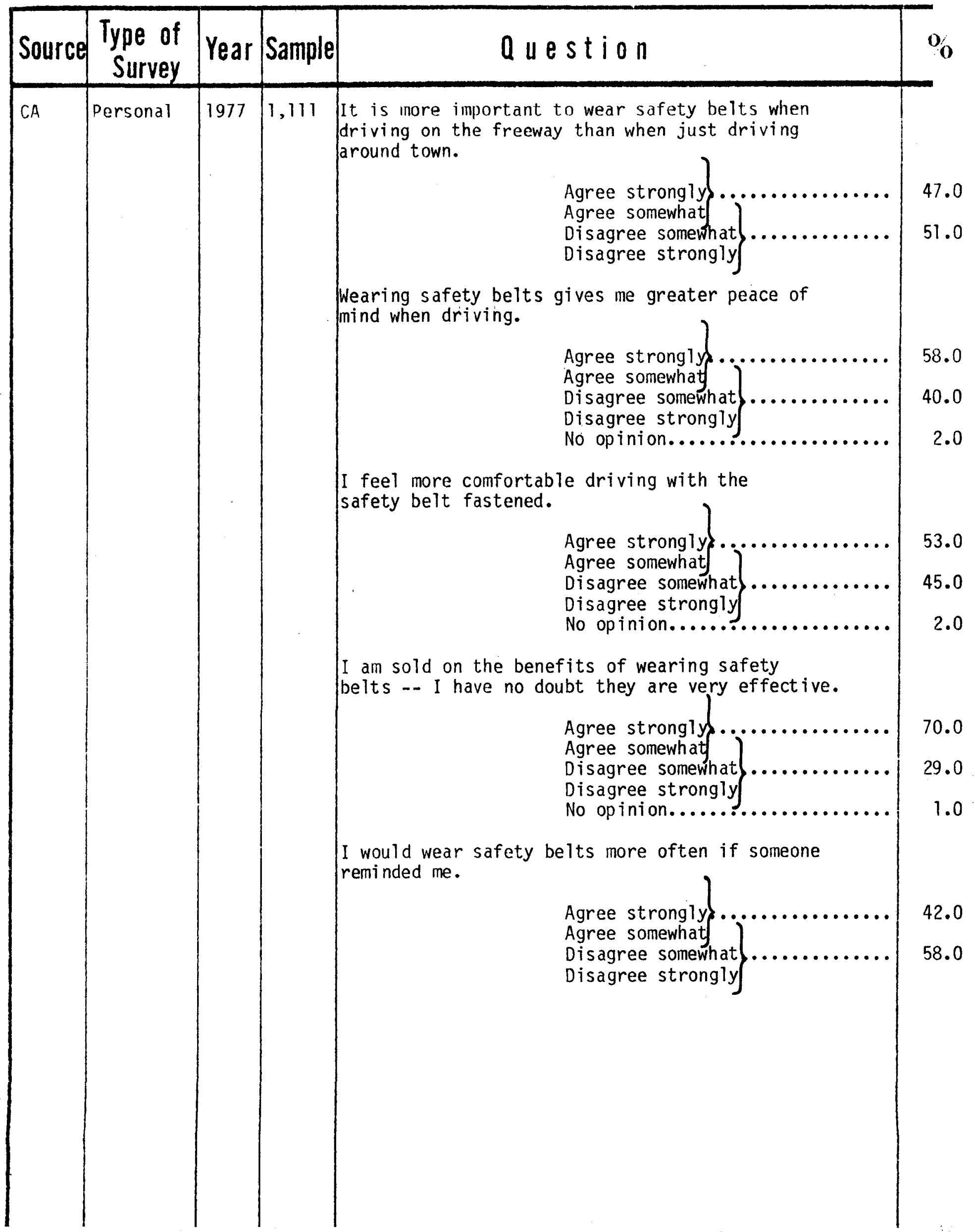




\begin{tabular}{|c|c|c|c|c|c|}
\hline Source & $=\begin{array}{c}\text { Type of } \\
\text { Survey }\end{array}$ & Year & Sample & Question & $\%$ \\
\hline$C A$ & Personal & 1977 & 1,111 & 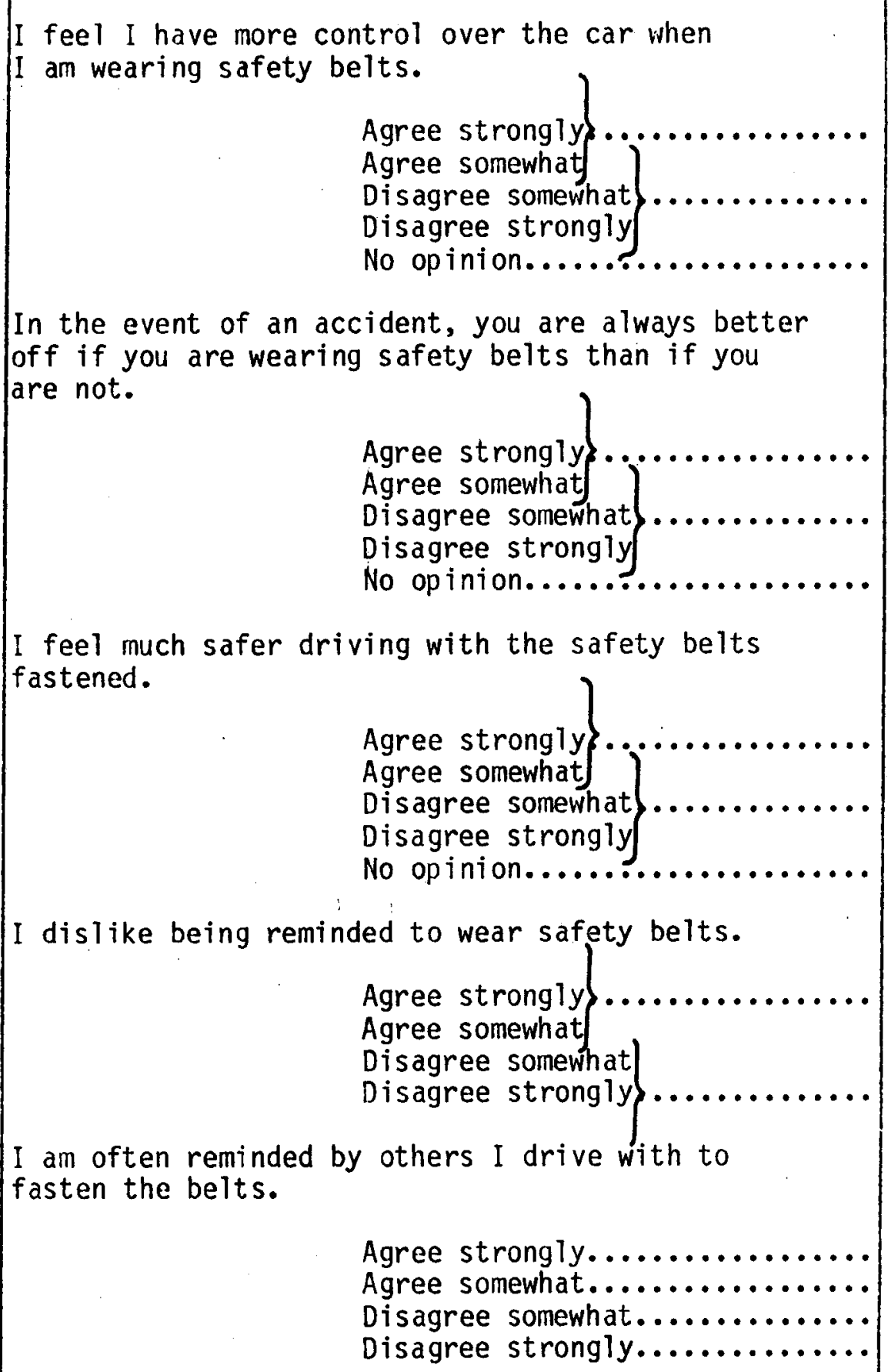 & $\begin{array}{r}46.0 \\
52.0 \\
2.0 \\
\\
67.0 \\
31.0 \\
2.0 \\
61.0 \\
38.0 \\
1.0 \\
44.0 \\
56.0\end{array}$ \\
\hline
\end{tabular}




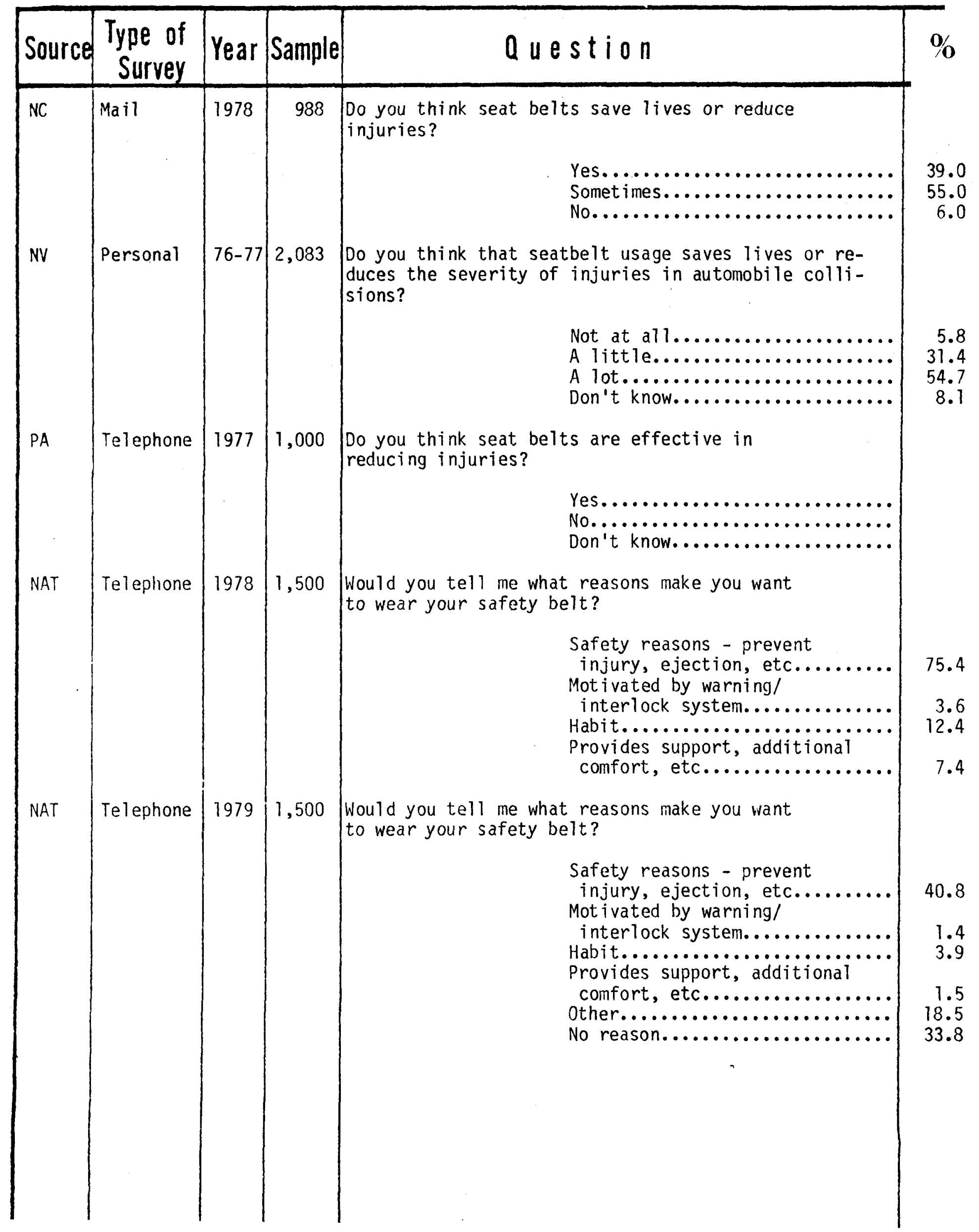




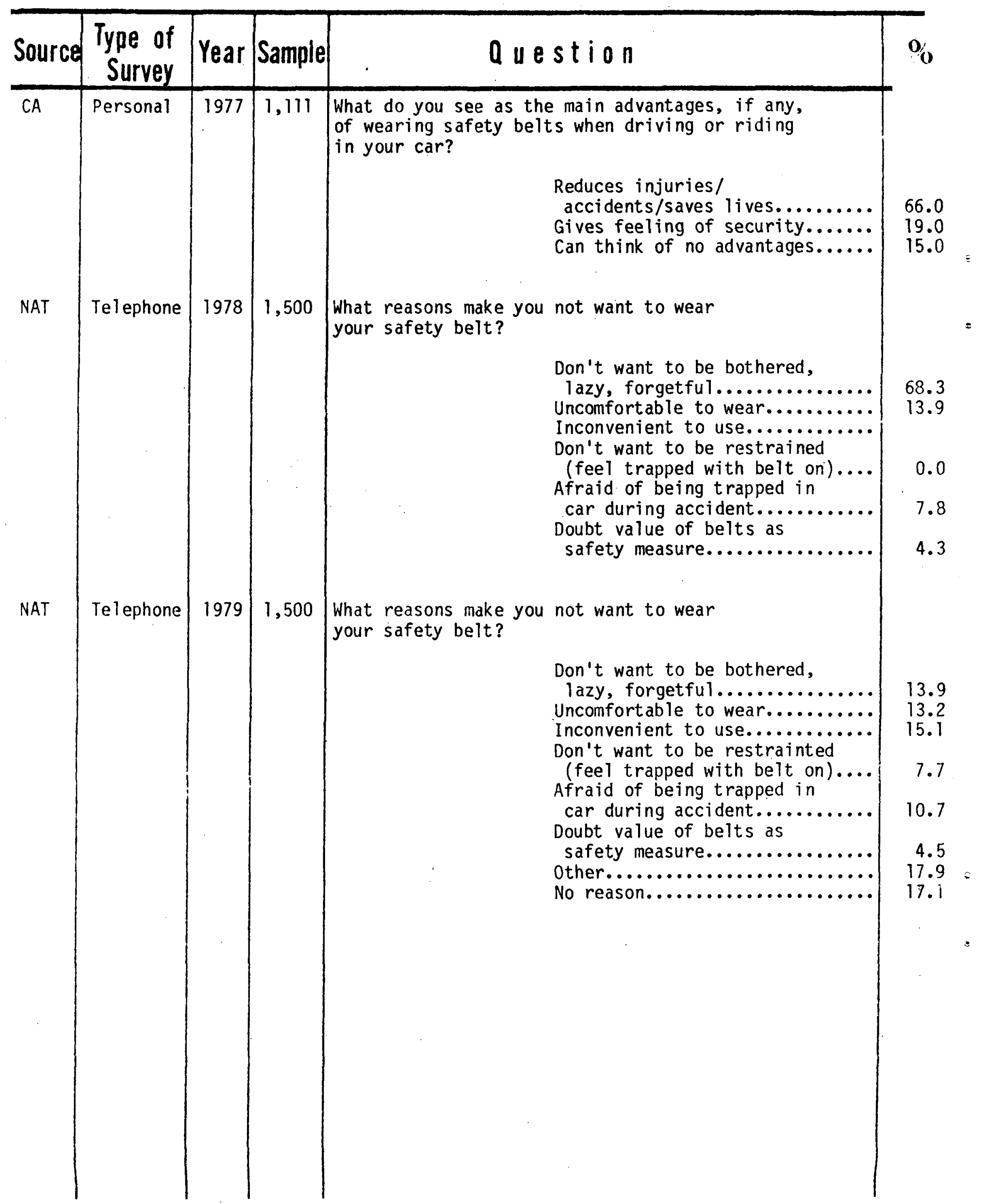




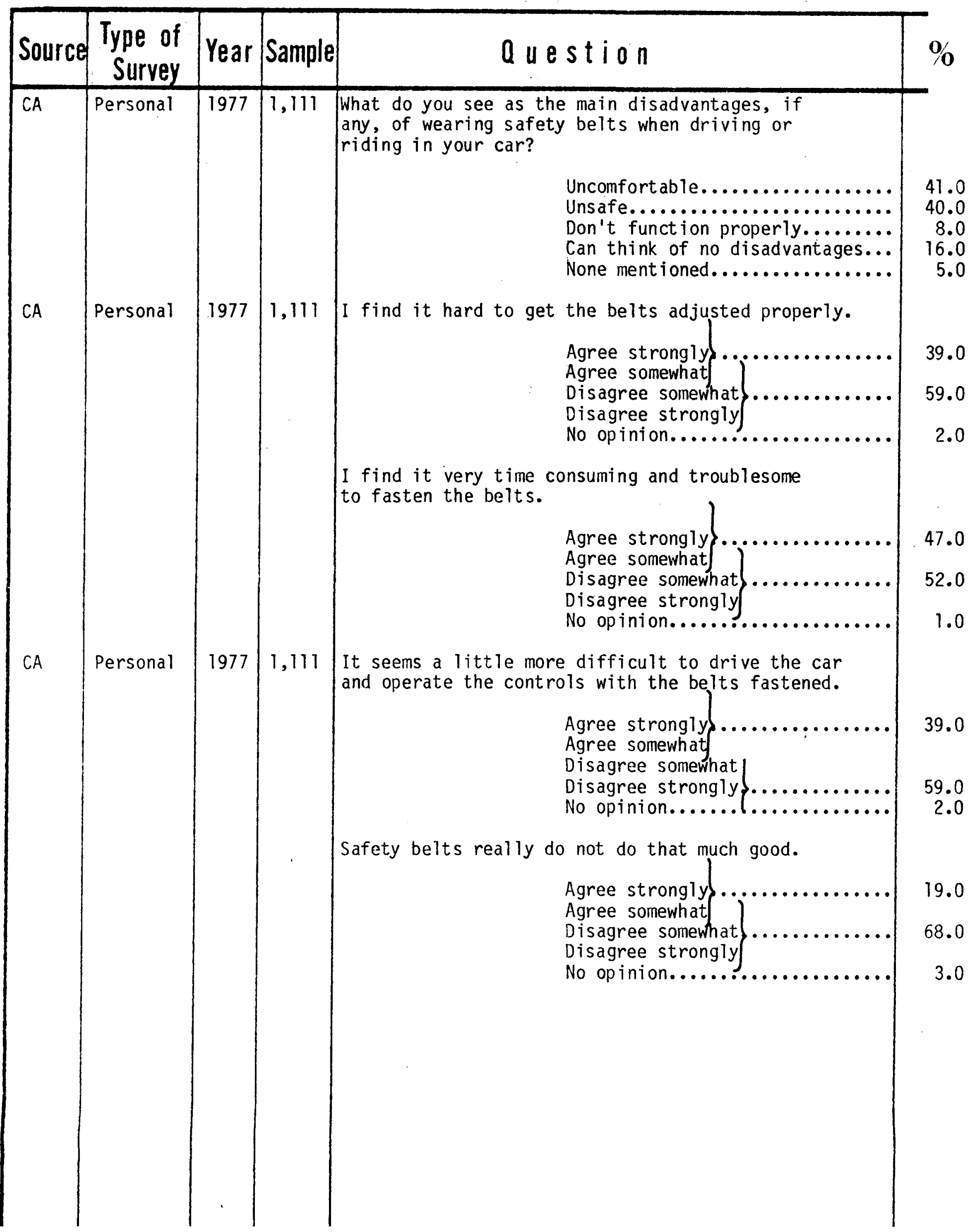




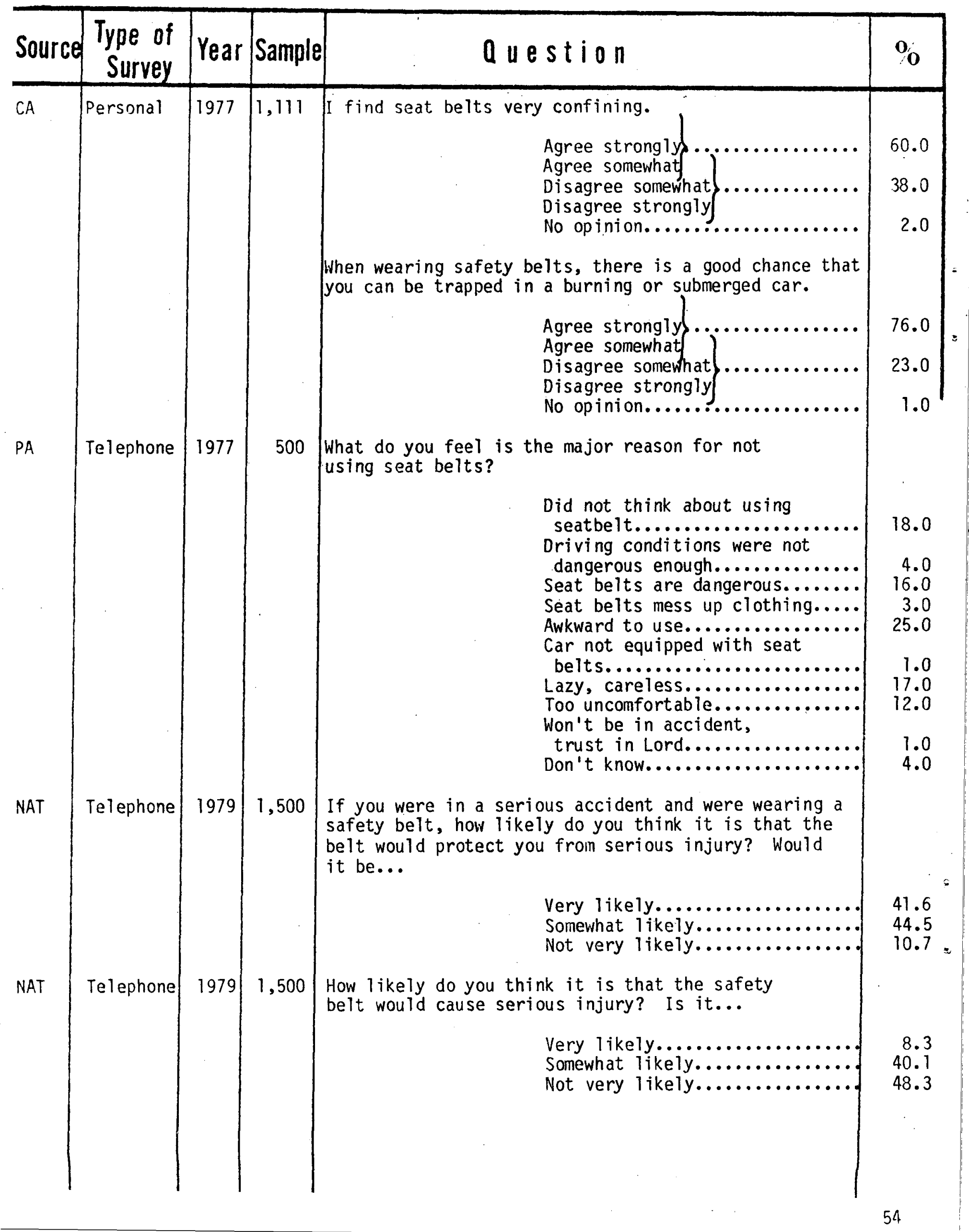




\begin{tabular}{|c|c|c|c|c|}
\hline Source & $\begin{array}{l}\text { Type of } \\
\text { Survey }\end{array}$ & Year & Sample & Q uestion \\
\hline NAT & Telephone & 1979 & 1,500 & 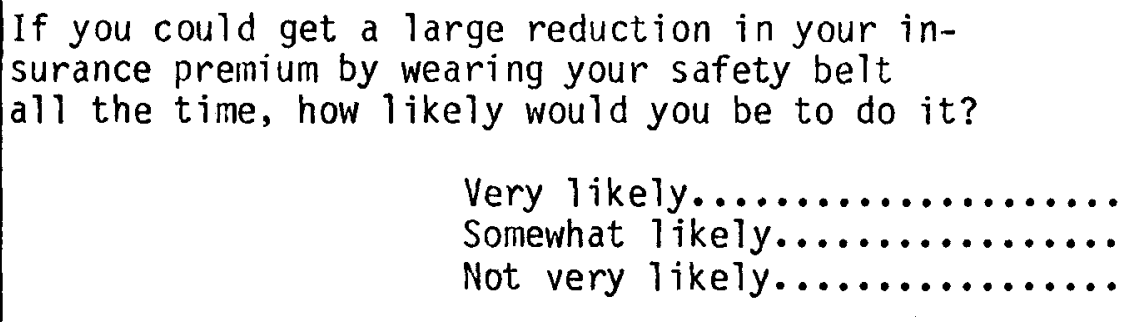 \\
\hline$C A$ & Personal & 1977 & 1,111 & 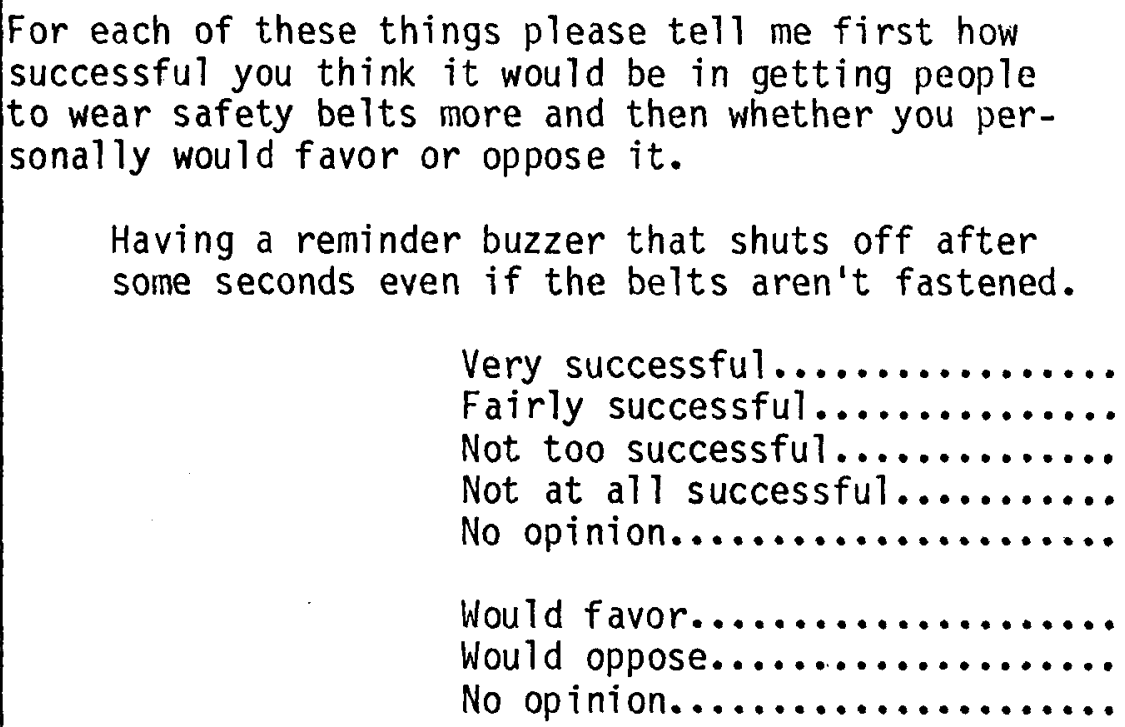 \\
\hline
\end{tabular}

75.8

12.9

9.8

Having an ignition lock system so that people cannot start the car until the belts are fastened.

Very successful.............. Fairly successful.............. Not too successful............. Not at all successful.......... No opinion..................

Would favor. Would oppose....................

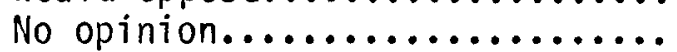




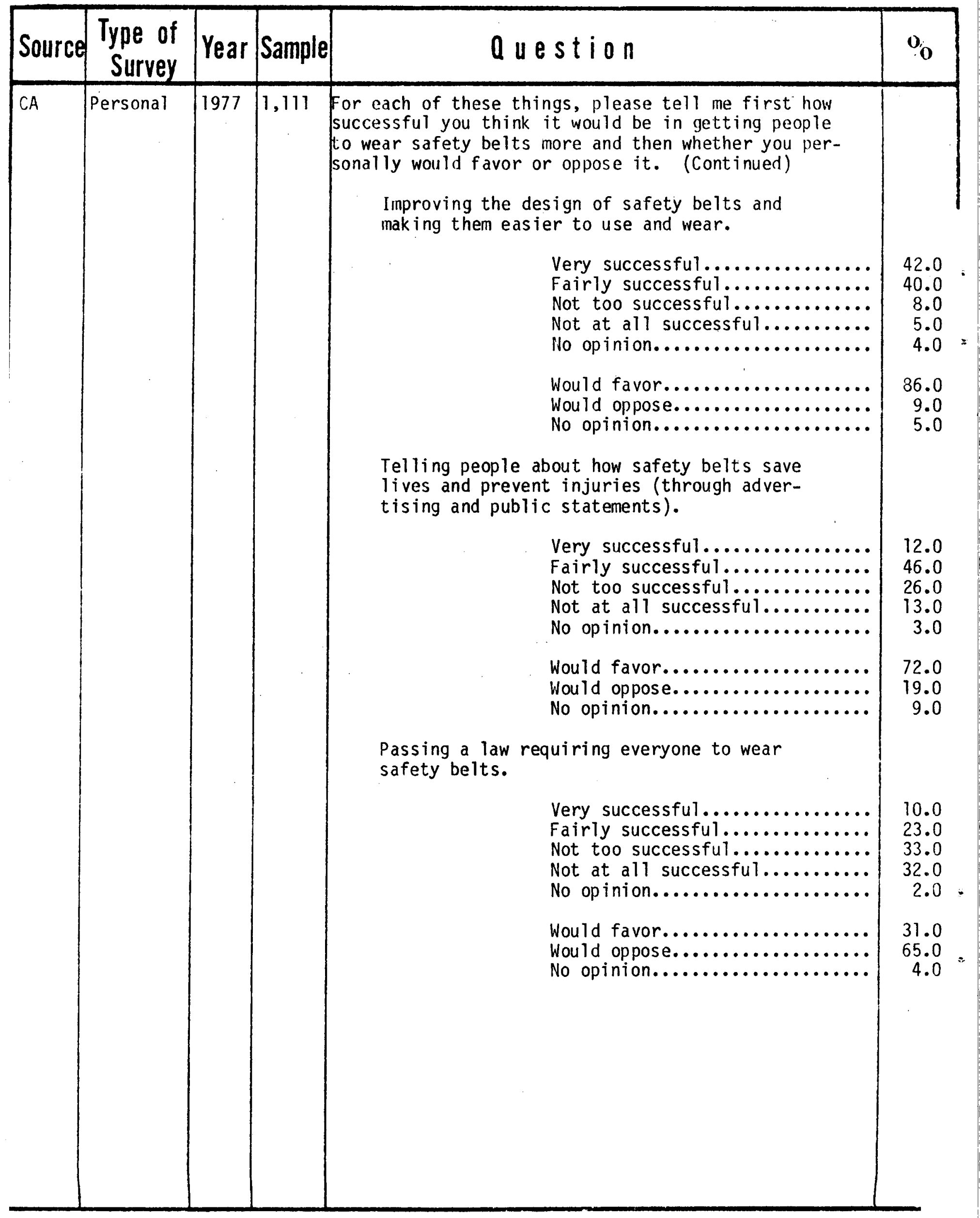




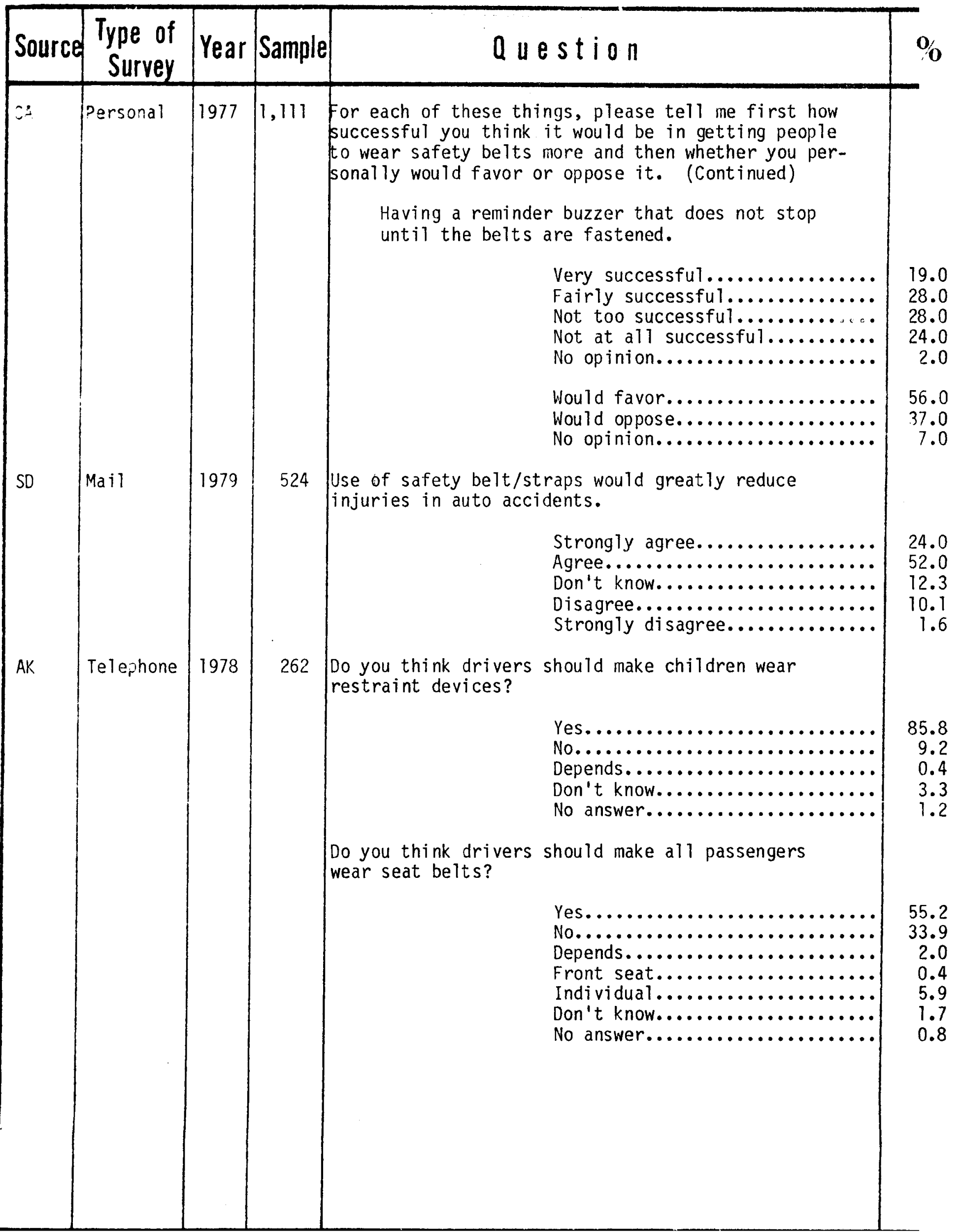




\begin{tabular}{|c|c|c|c|c|c|}
\hline Source & $\begin{array}{l}\text { Type of } \\
\text { Survey }\end{array}$ & Year & Sample & Question & $\%$ \\
\hline NAT & Tel ephone & 1979 & 1,500 & 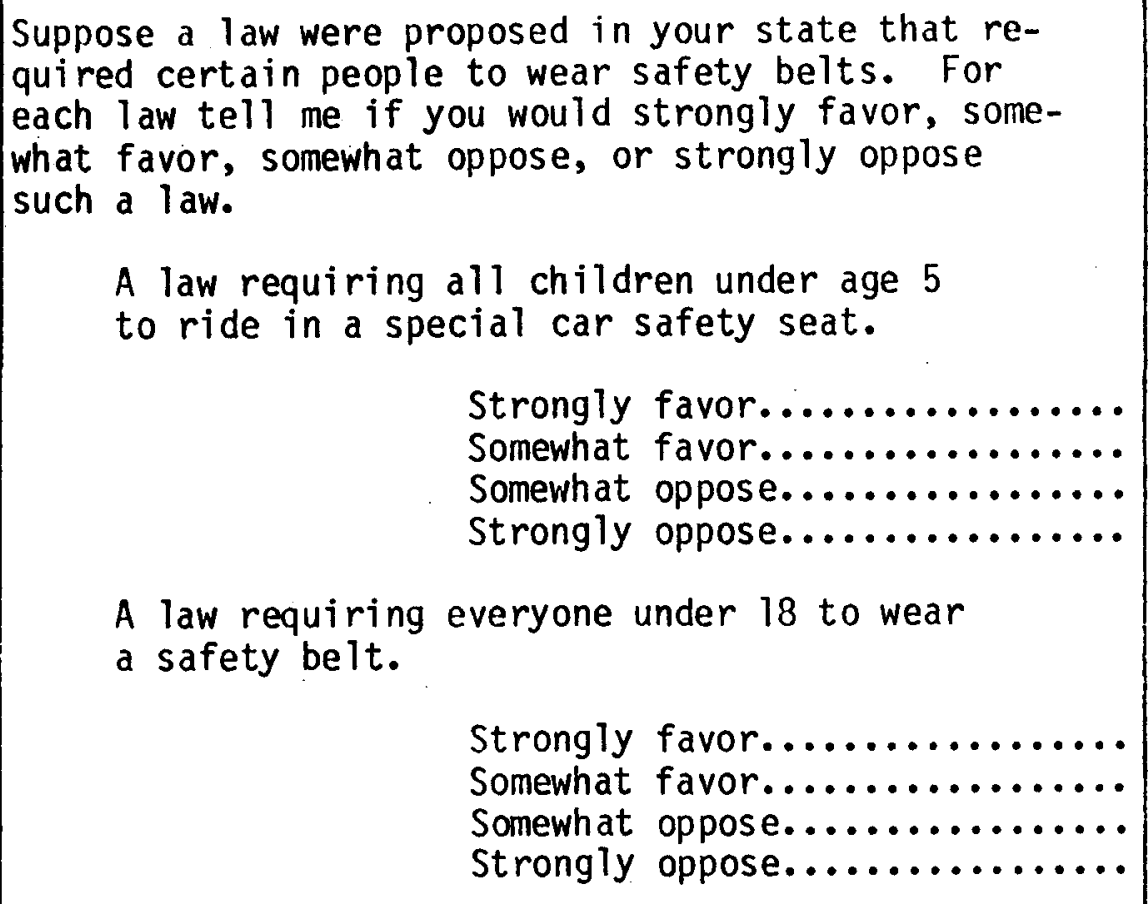 & $\begin{array}{r}65.0 \\
18.7 \\
7.6 \\
8.0 \\
\\
\\
32.7 \\
27.7 \\
21.4 \\
17.1\end{array}$ \\
\hline NAT & Telephone & 1978 & 1,500 & 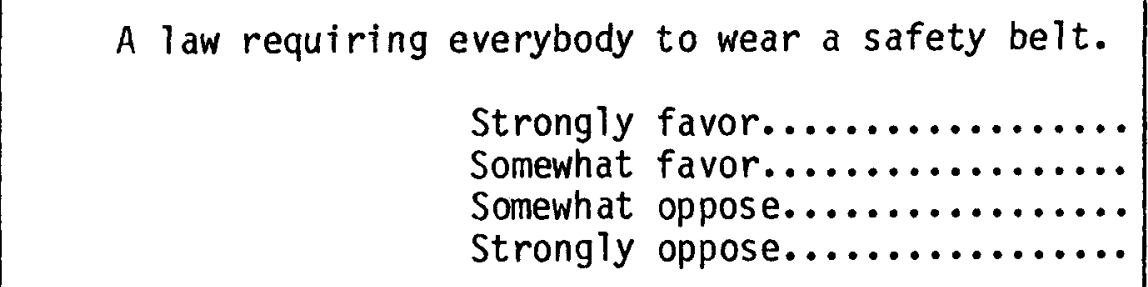 & $\begin{array}{l}20.8 \\
33.2 \\
21.3 \\
23.7\end{array}$ \\
\hline NAT & Telephone & 1979 & 1,500 & 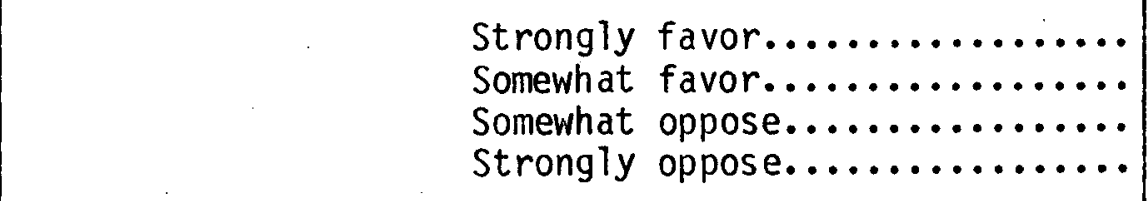 & $\begin{array}{l}29.1 \\
22.9 \\
18.8 \\
28.1\end{array}$ \\
\hline NC & Mail & 1978 & 988 & 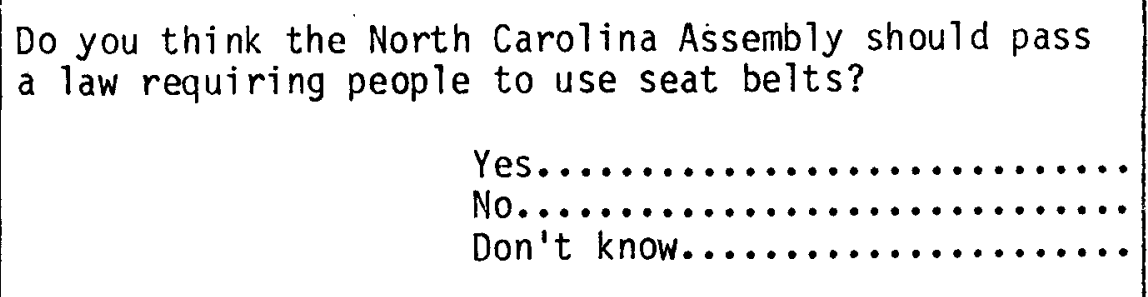 & $\begin{array}{l}14.0 \\
62.0 \\
23.0\end{array}$ \\
\hline NV & Personal & 1977 & 1,767 & 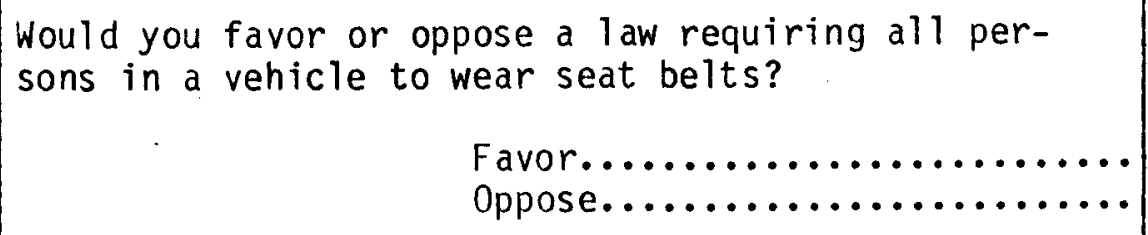 & $\begin{array}{l}28.5 \\
71.5\end{array}$ \\
\hline
\end{tabular}




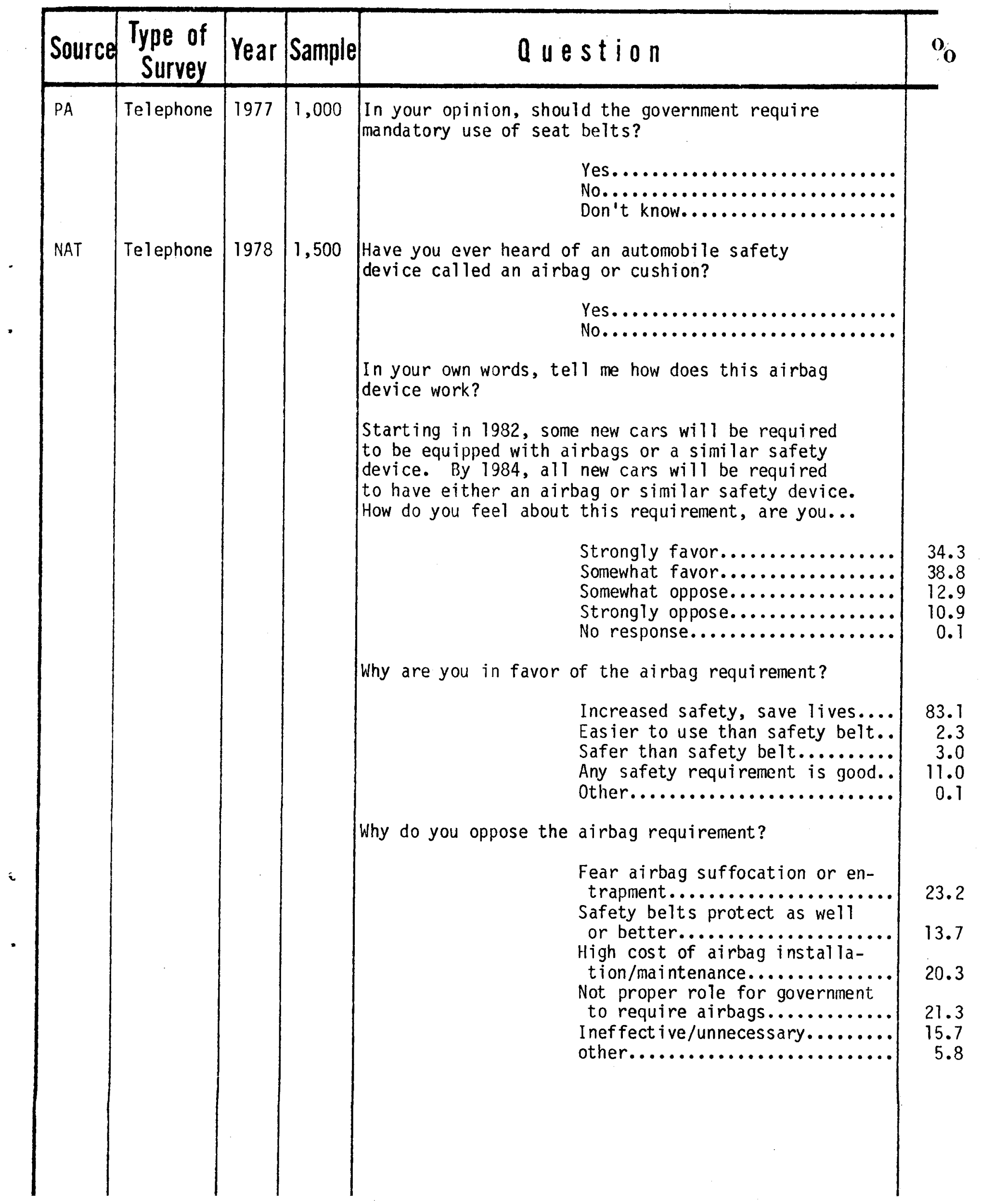




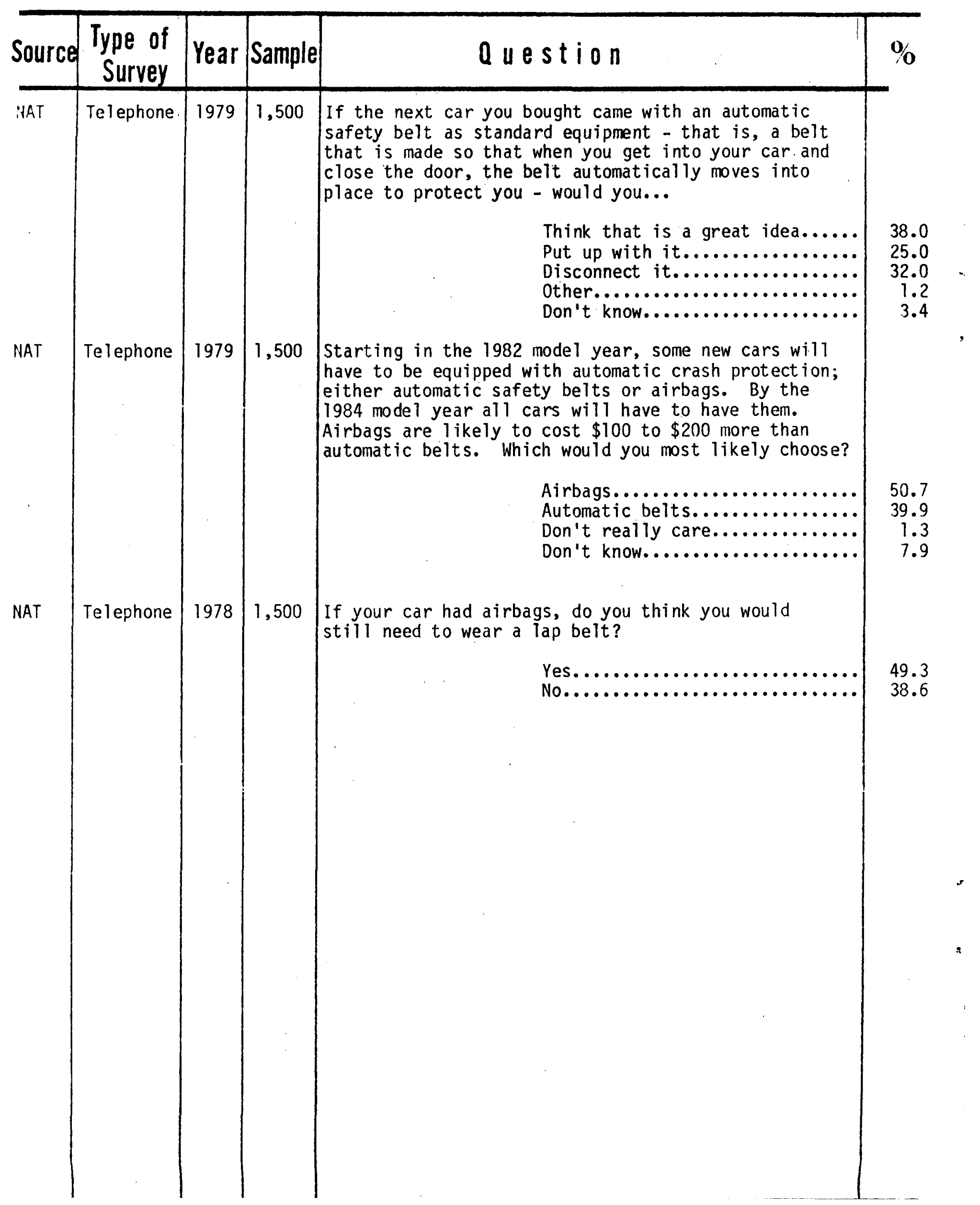




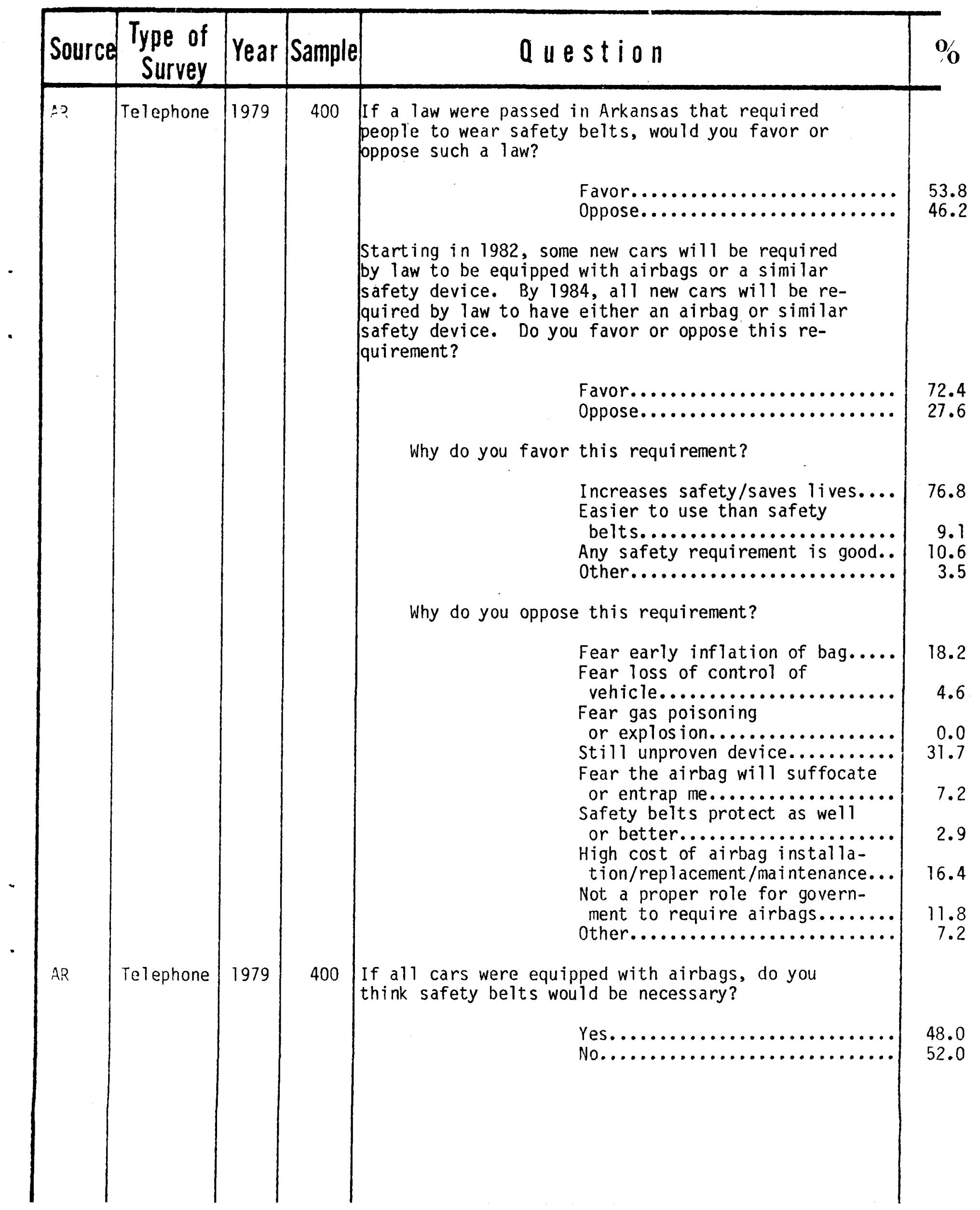




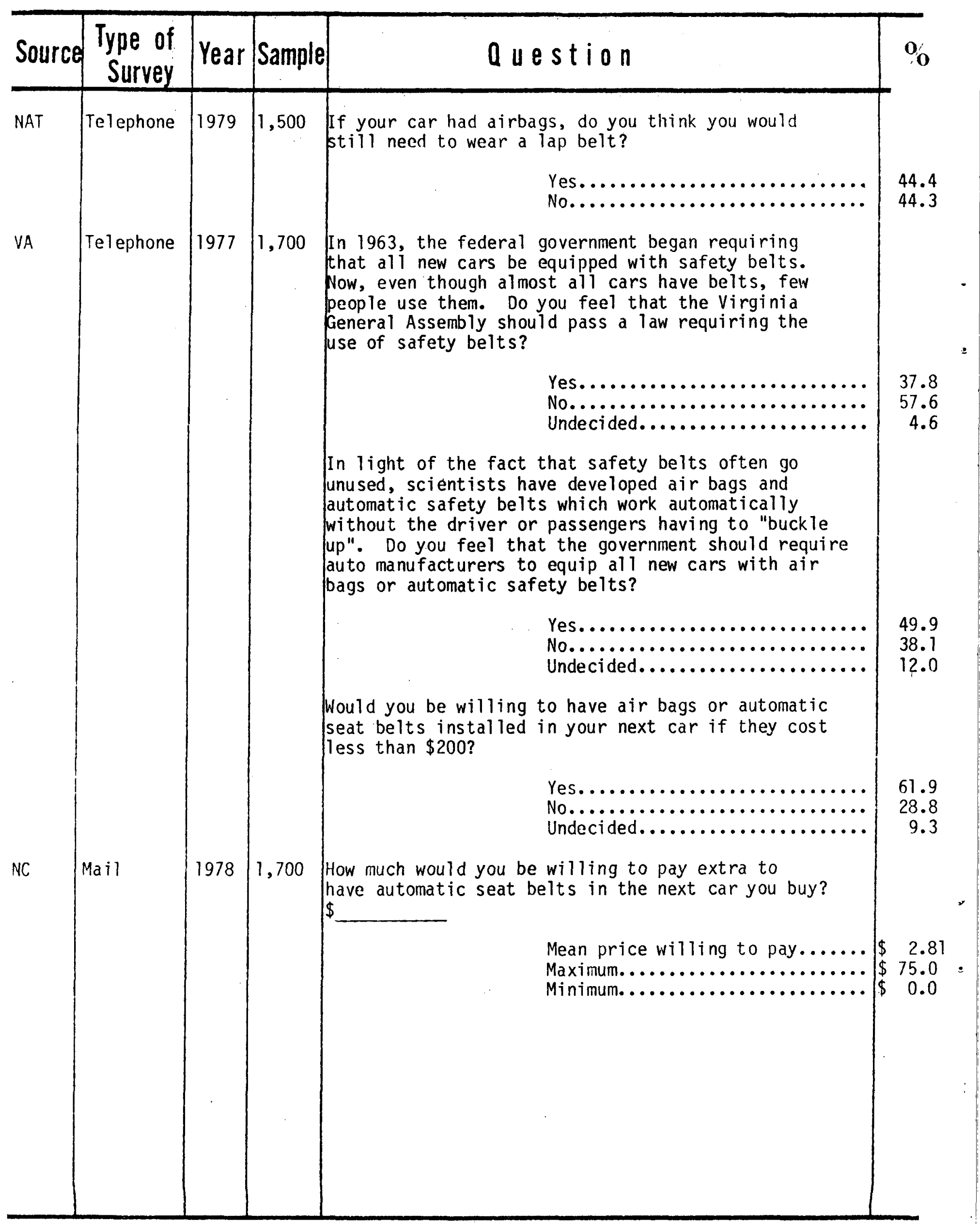


BEHAVIOR $\longrightarrow$ 
SB-B-1

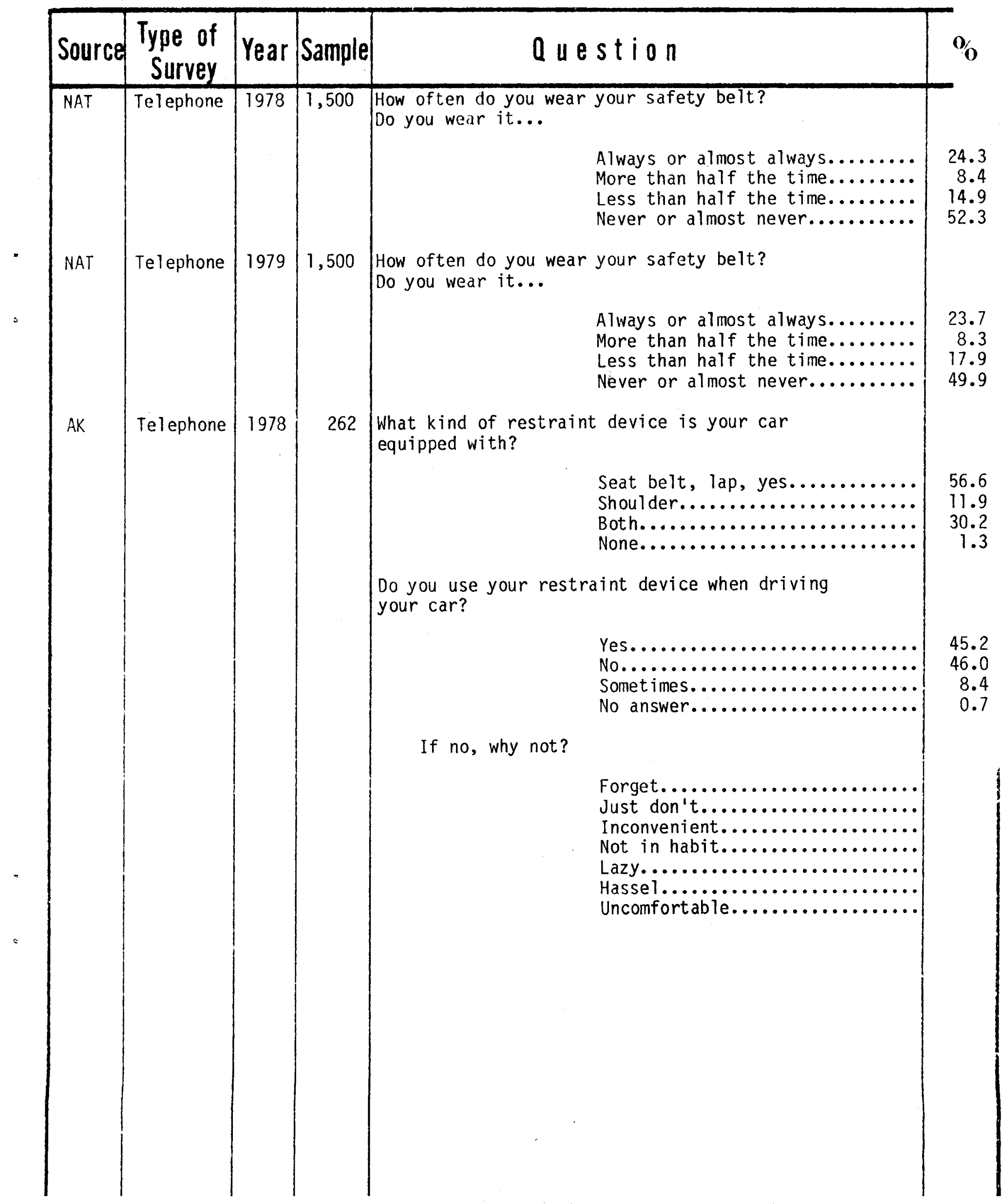




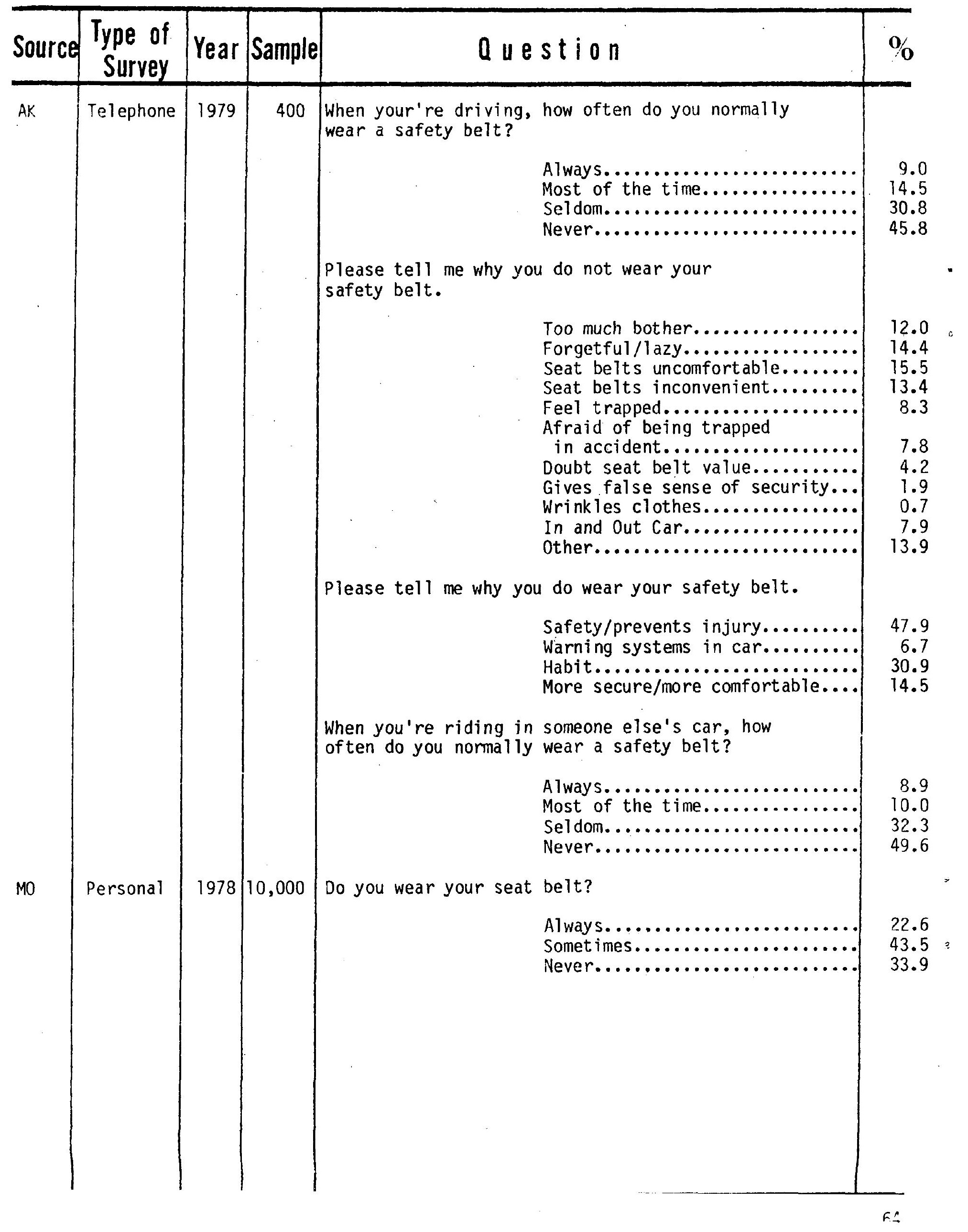




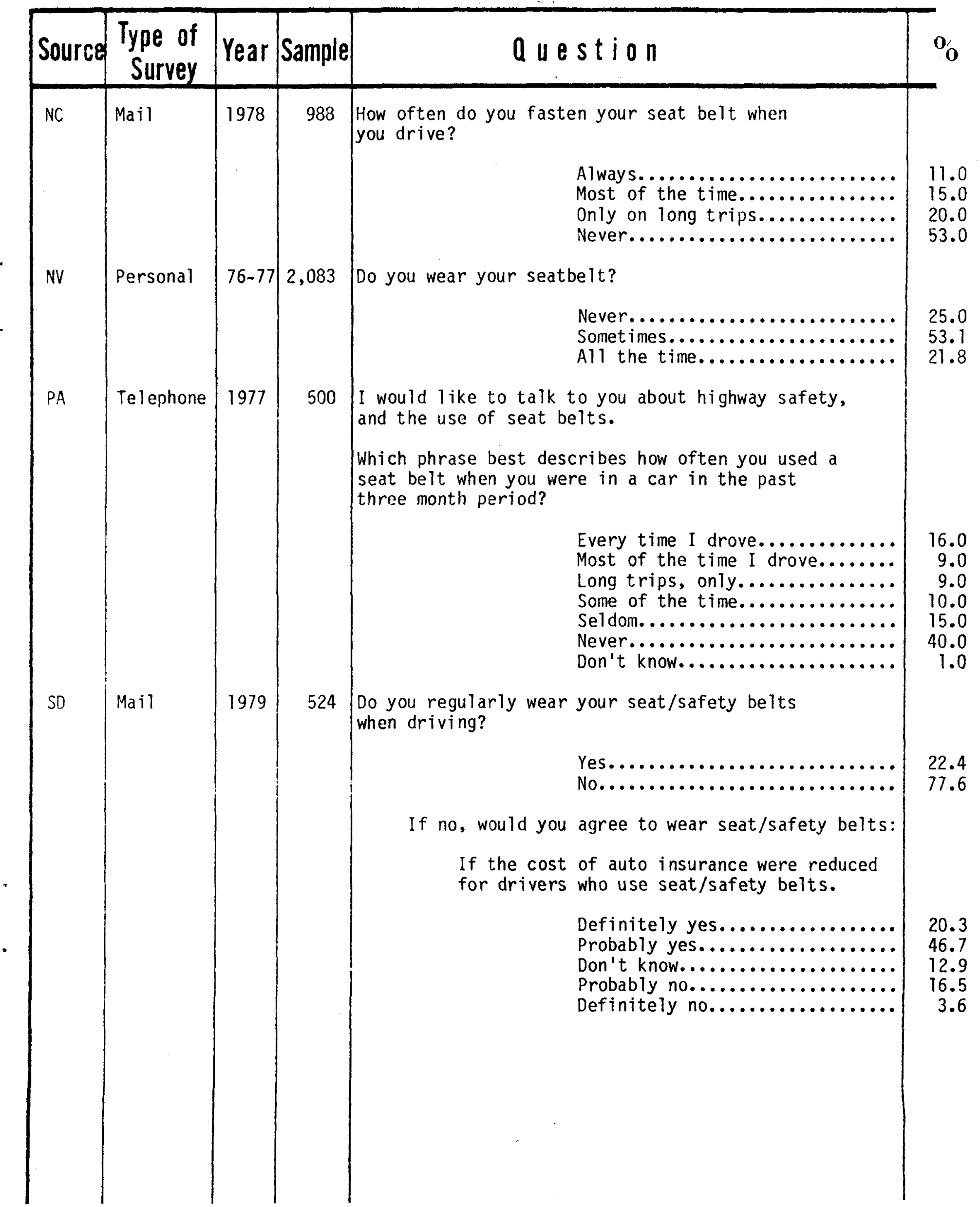




\begin{tabular}{|c|c|c|c|c|}
\hline Source & $\begin{array}{l}\text { Type of } \\
\text { Survey }\end{array}$ & Year & Sample & Question \\
\hline 5 & Yail & 1979 & 524 & 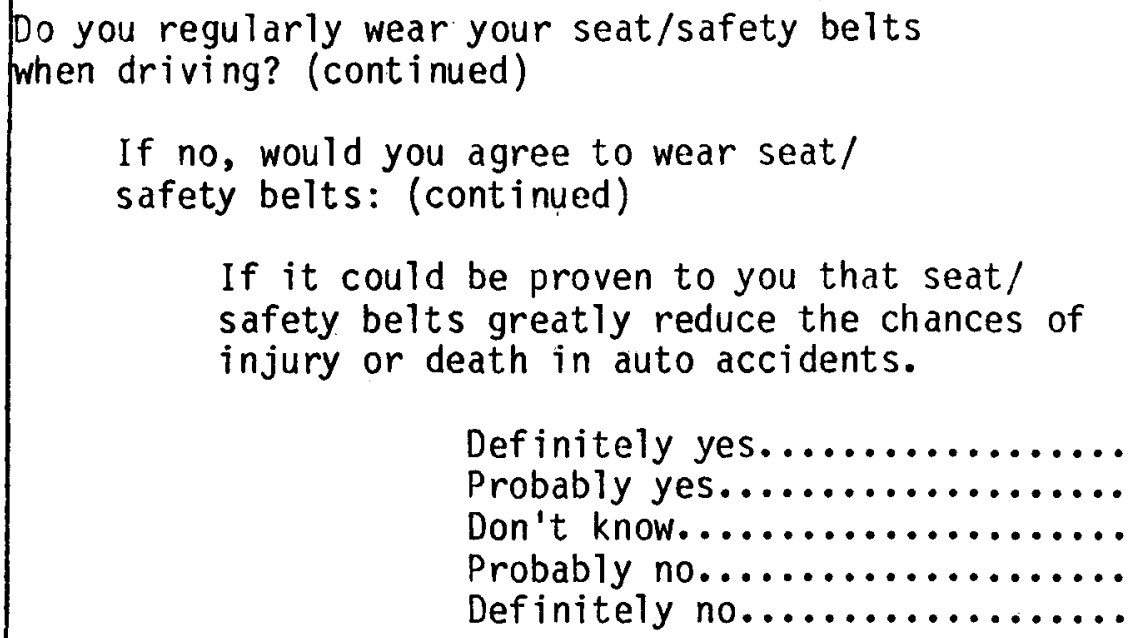 \\
\hline
\end{tabular}

O

If the seat/safety belts were made more convenient and comfortable.

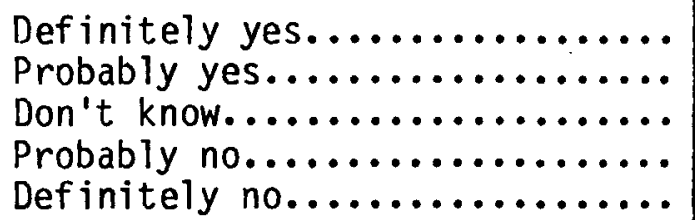

22.2

49.7

13.0

12.8

2.3

If a 1 aw were passed to make the wearing of seat/safety belts mandatory for all occupants of the car.

Definitely yes...............

Probably yes..................

Don't know...................

Definitely no................

21.7

38.0

11.7

8.4

If a mandatory device (such as a buzzer) were installed on your car to remind you that seat/safety belts were not fastened.

Definitely yes..............

Probably yes.................

Don't know..................

Probably no..................

Definitely no................ 


\begin{tabular}{|c|c|c|c|c|c|}
\hline Source & $\begin{array}{l}\text { Type of } \\
\text { Survey }\end{array}$ & Year & Sample & Question & o \\
\hline$C A$ & Personal & 1977 & $\mid$ & 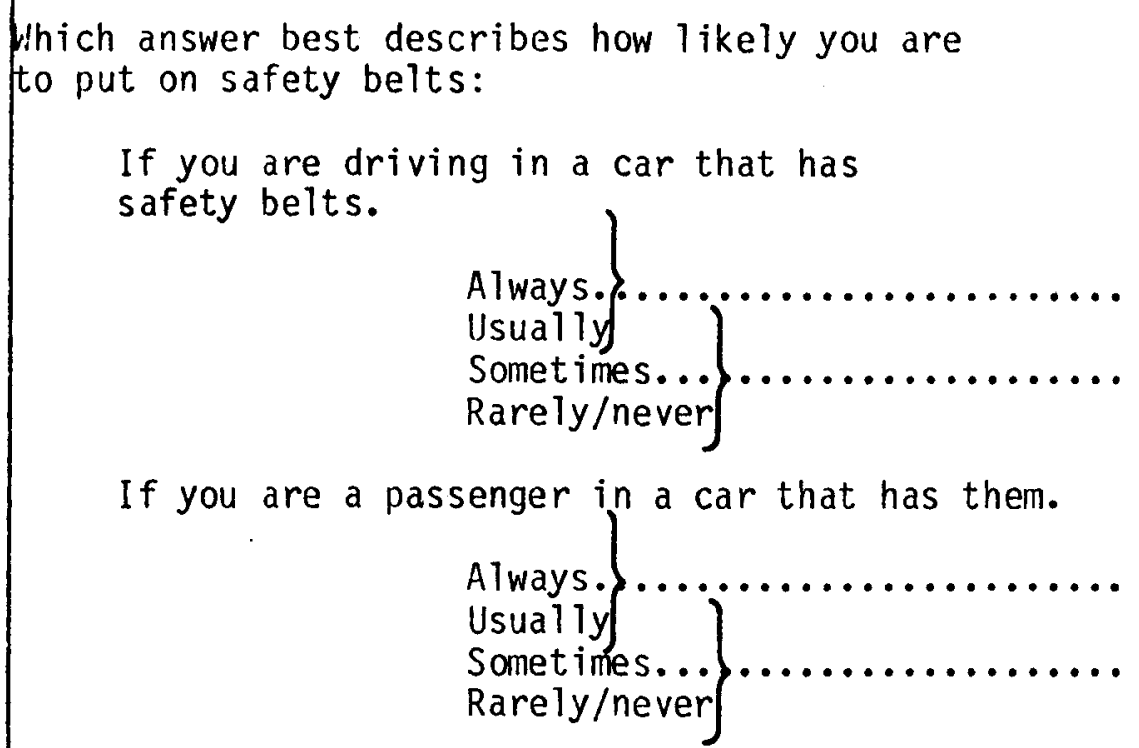 & $\begin{array}{l}81.0 \\
67.0 \\
70.0 \\
59.0\end{array}$ \\
\hline$C A$ & Personal & 1977 & 1,111 & 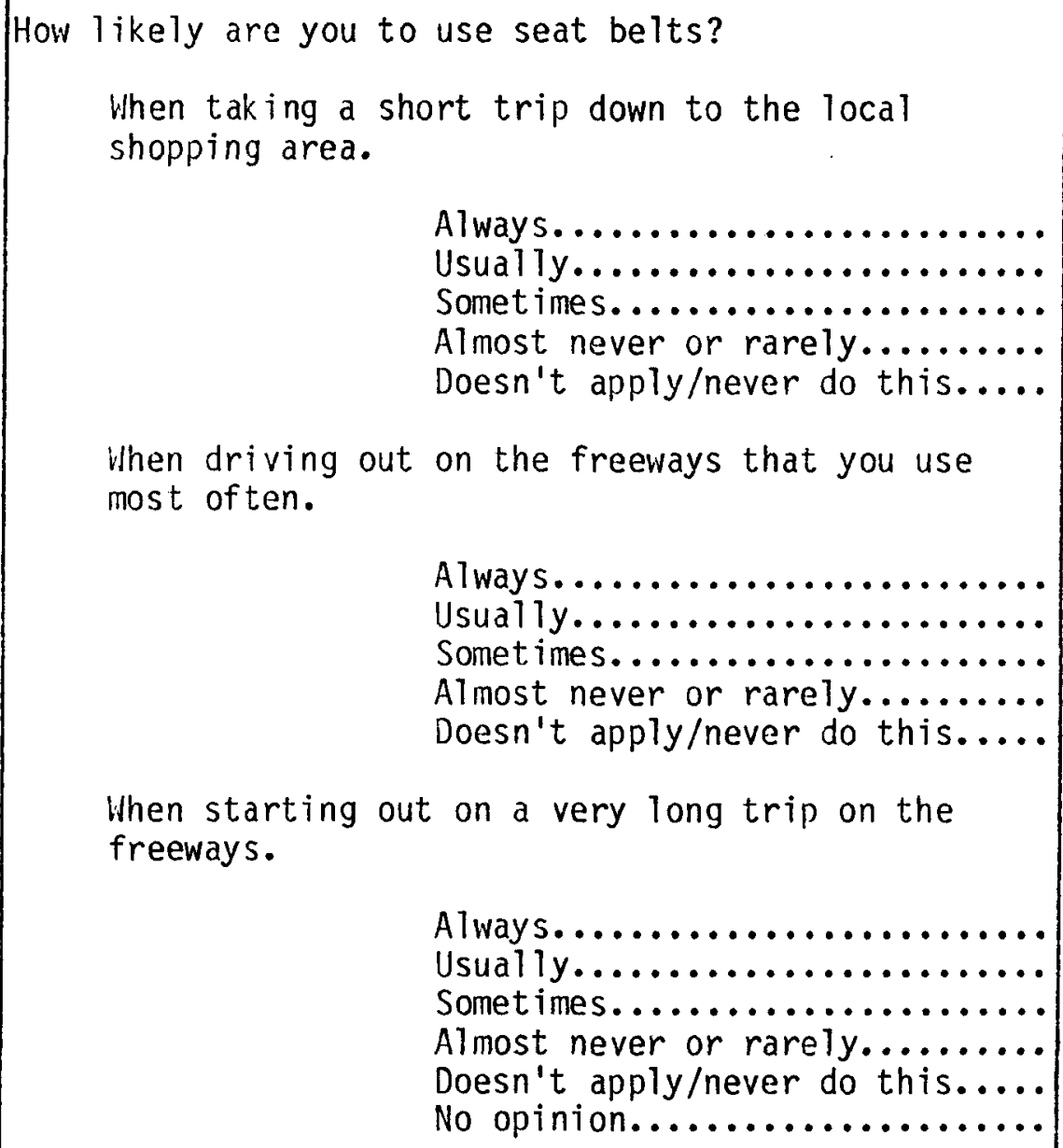 & $\begin{array}{r} \\
\\
35.0 \\
11.0 \\
13.0 \\
36.0 \\
5.0 \\
\\
\\
\\
55.0 \\
19.0 \\
13.0 \\
10.0 \\
4.0 \\
\\
\\
\\
65.0 \\
17.0 \\
8.0 \\
5.0 \\
4.0 \\
1.0\end{array}$ \\
\hline
\end{tabular}




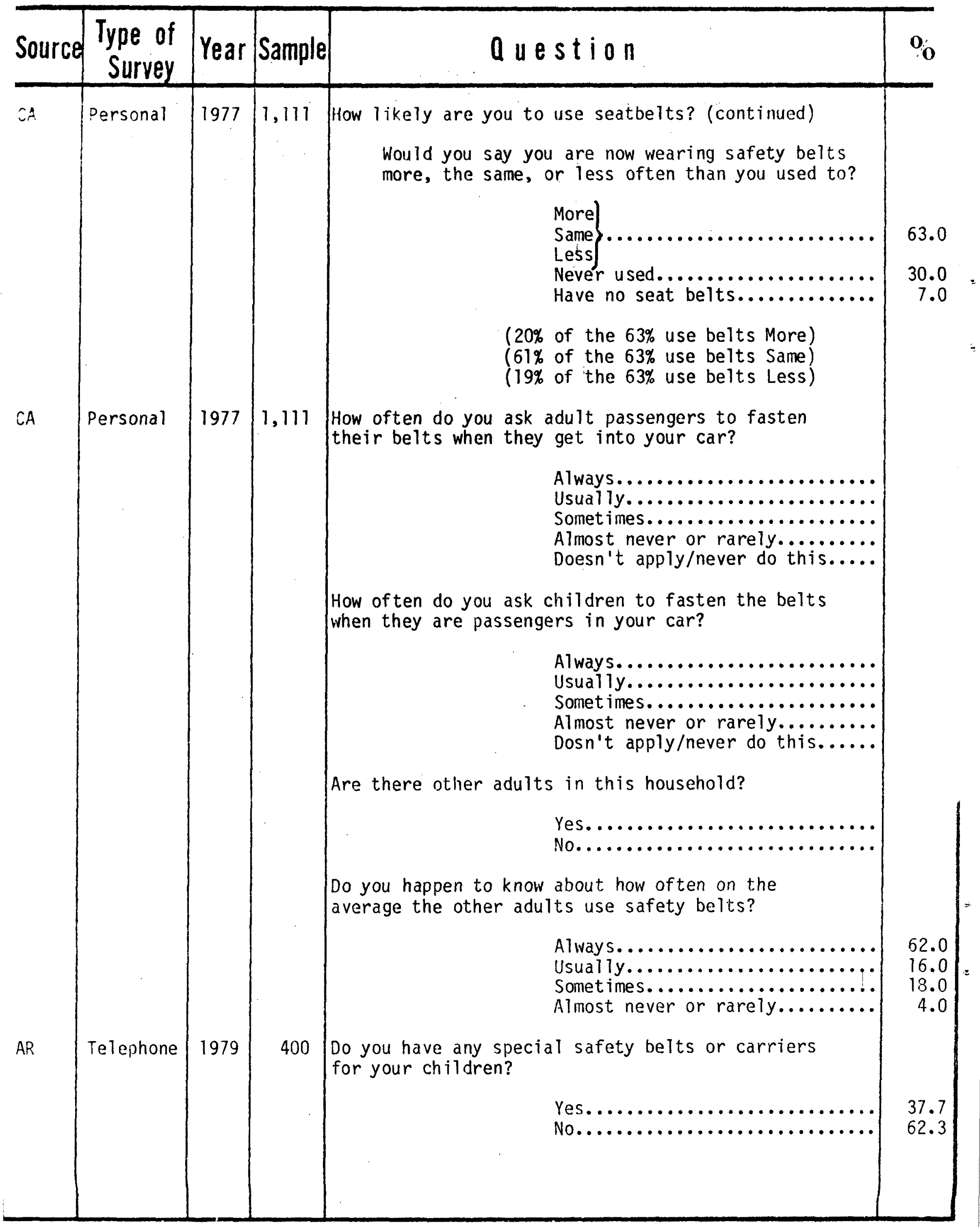




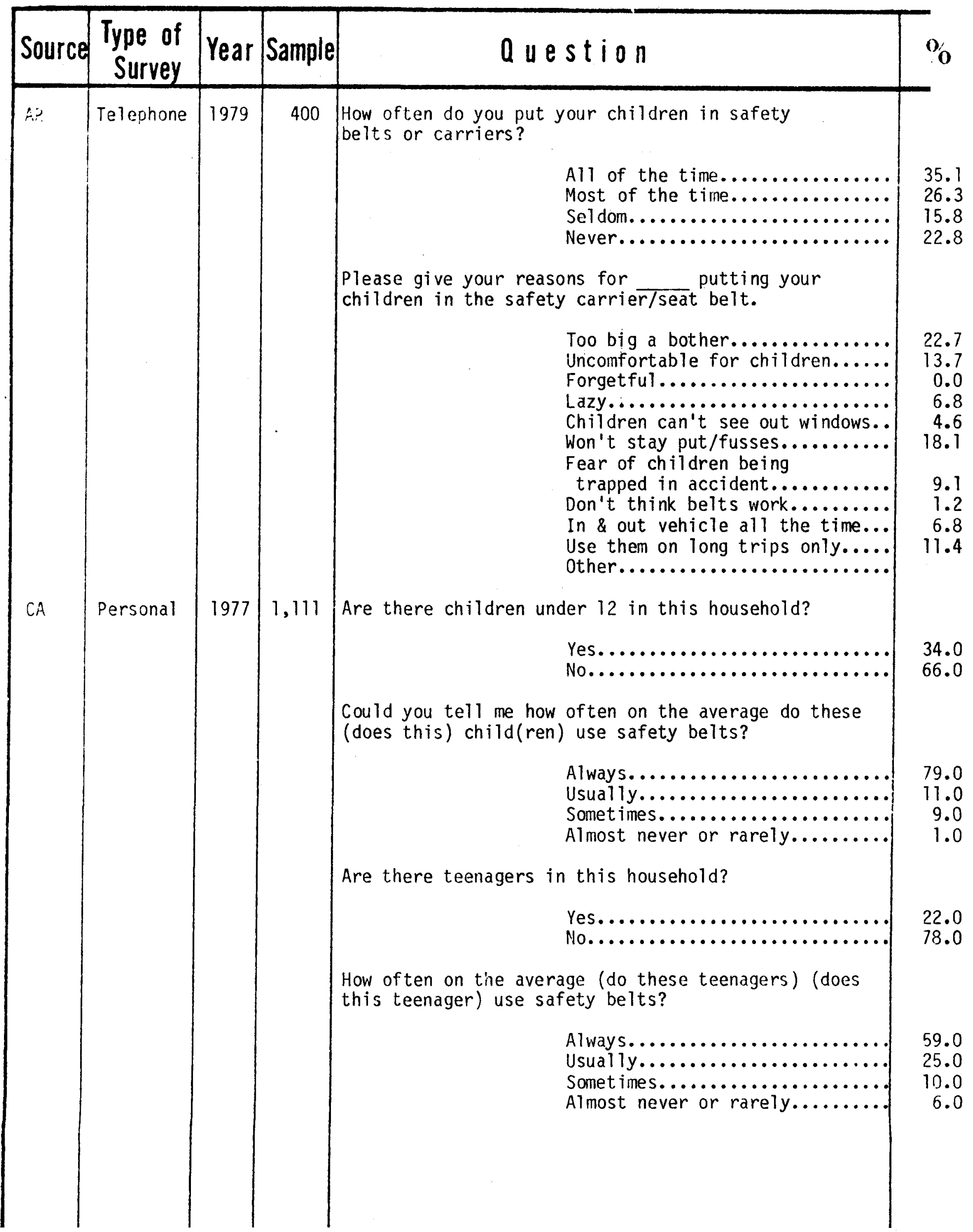




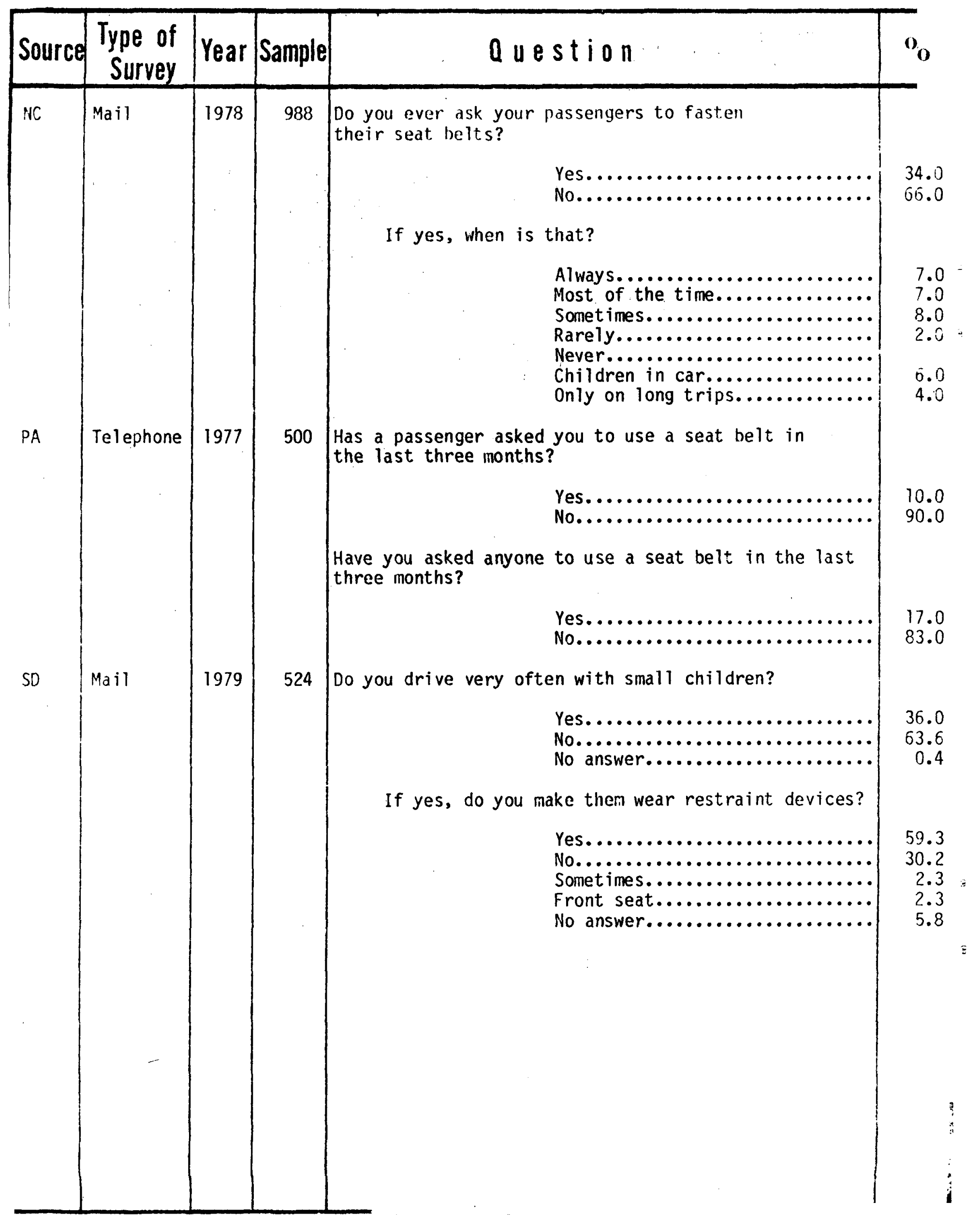


KNOWLEDGE

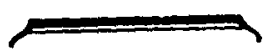




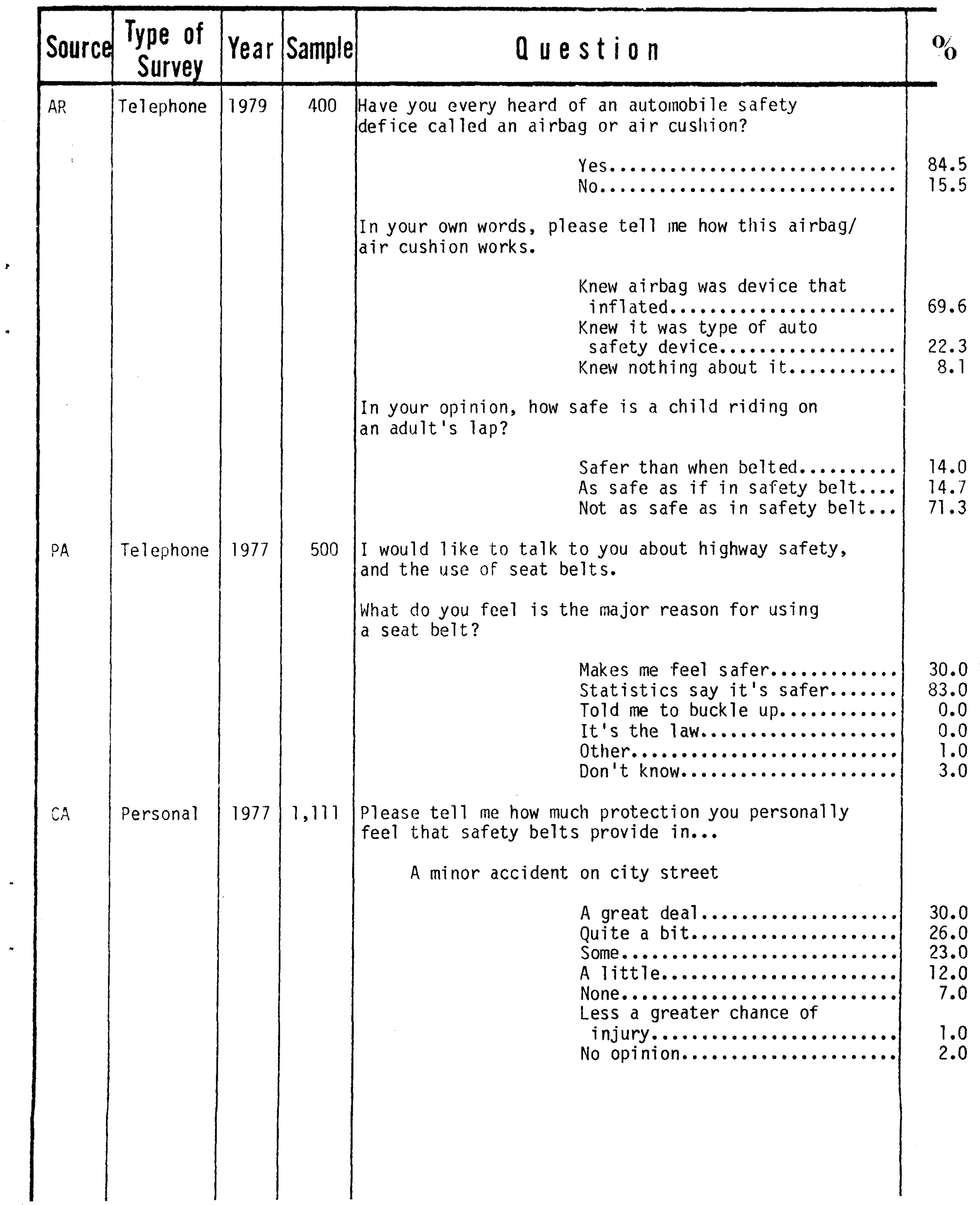




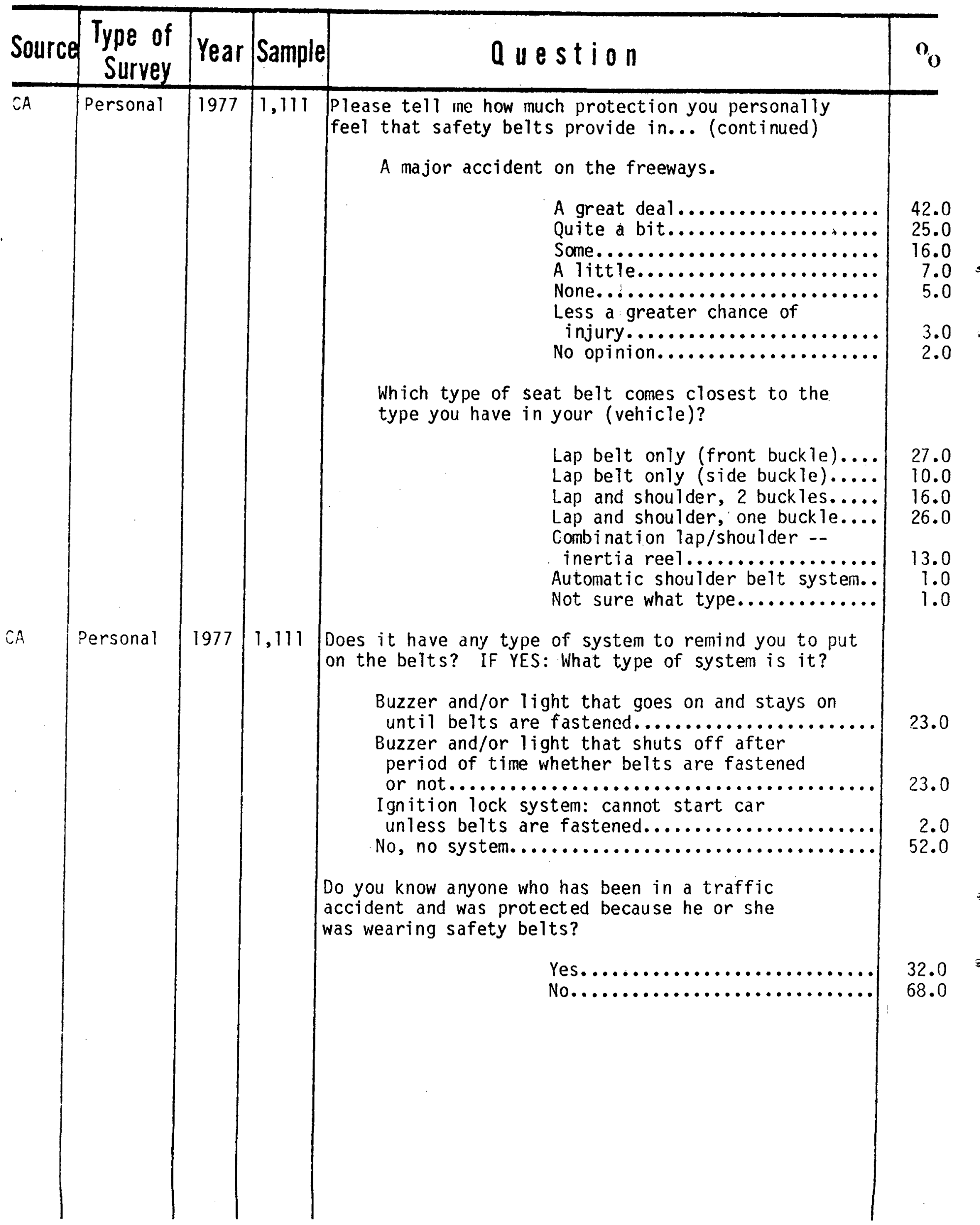




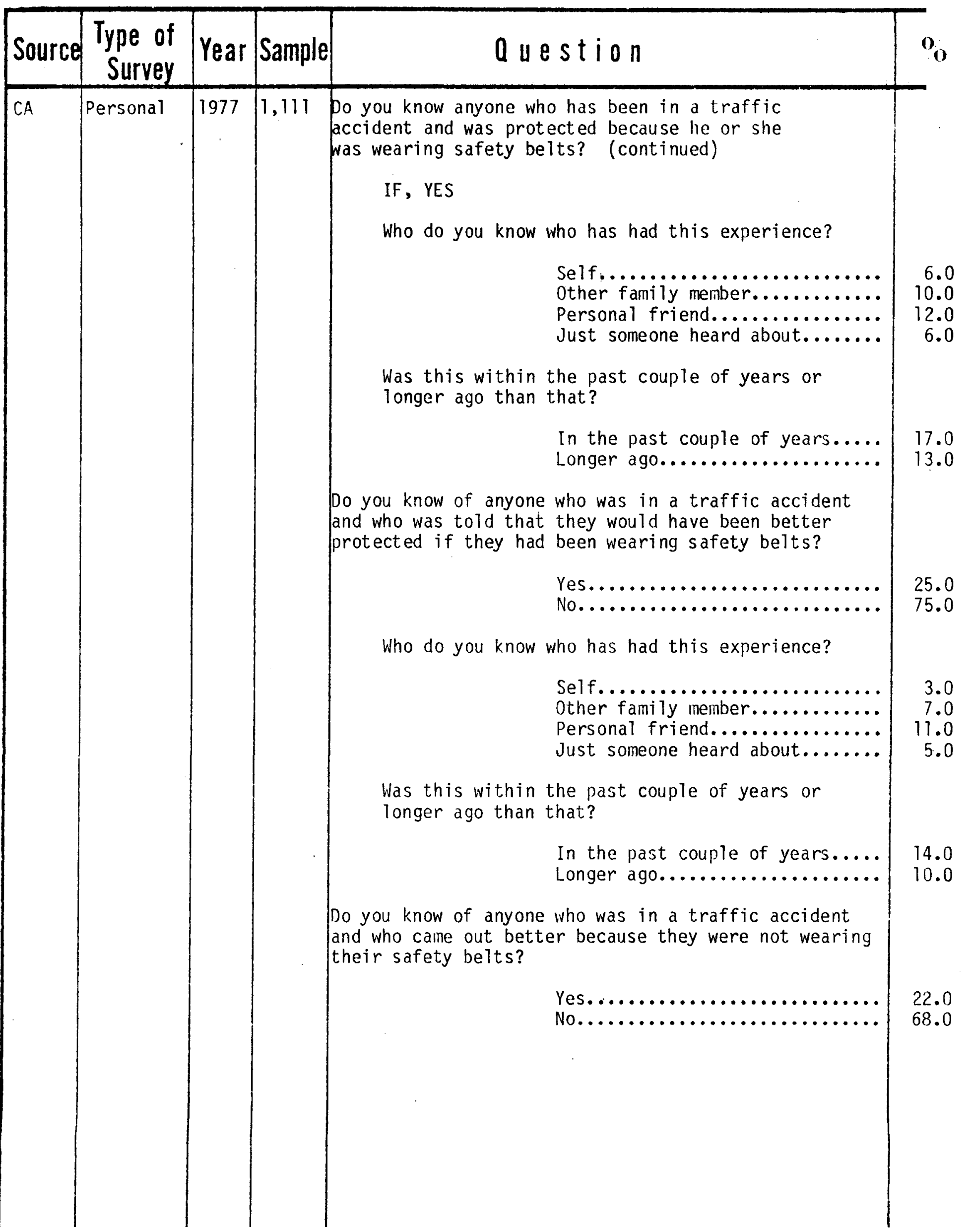


SB-K-4

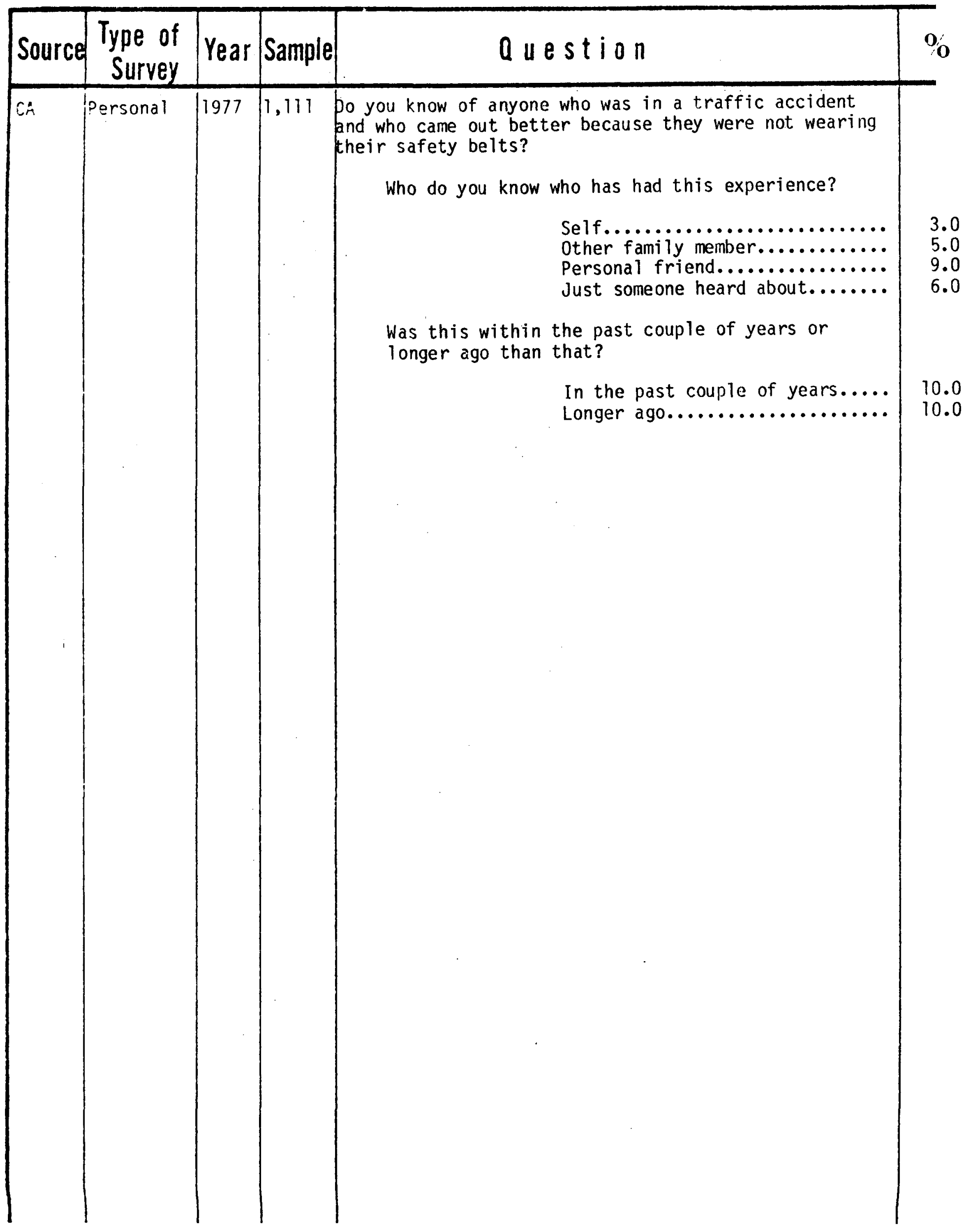

70 


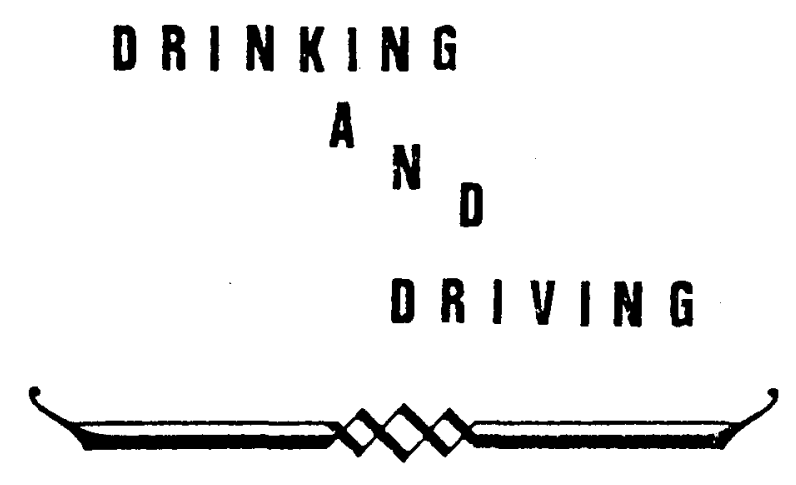


ATTITUDE $\longrightarrow$ 


\begin{tabular}{|c|c|c|c|c|c|}
\hline Source & $\begin{array}{c}\text { Type of } \\
\text { Survey }\end{array}$ & Year & Sample & Question & $\%$ \\
\hline MO & Personal & 1978 & 10,000 & 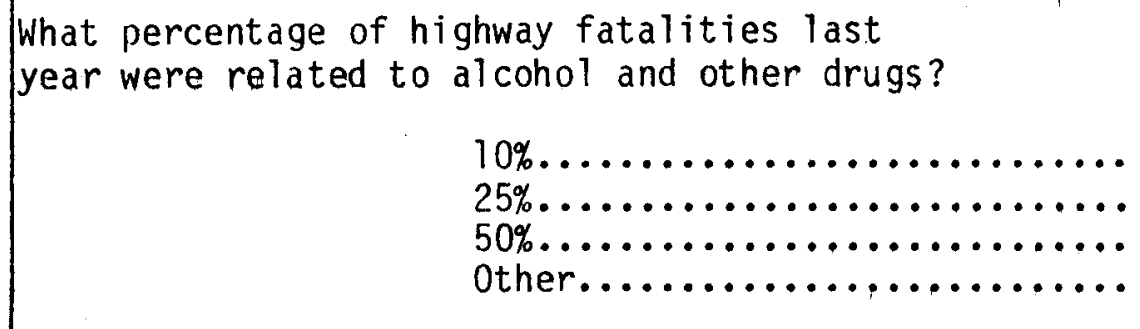 & $\begin{array}{r}11.5 \\
25.0 \\
54.0 \\
9.5\end{array}$ \\
\hline NC & Mail & 1978 & 988 & 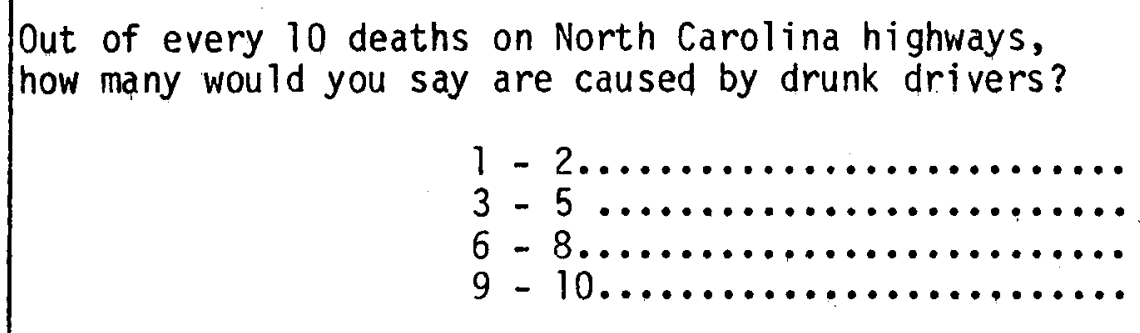 & $\begin{array}{r}12.0 \\
45.0 \\
32.0 \\
6.0\end{array}$ \\
\hline NC & Mail & 1978 & 988 & 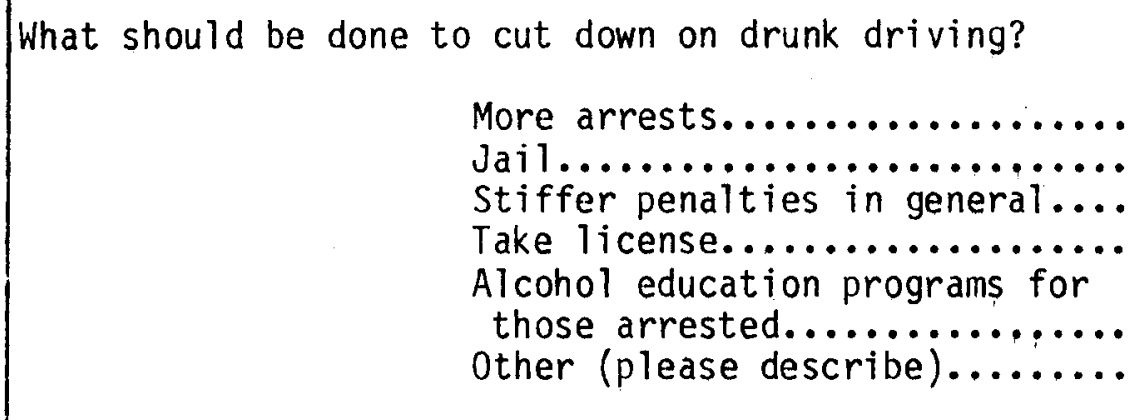 & $\begin{array}{r}10.0 \\
5.0 \\
49.0 \\
27.0 \\
27.0 \\
5.0\end{array}$ \\
\hline MO & Personal & 1978 & 10,000 & 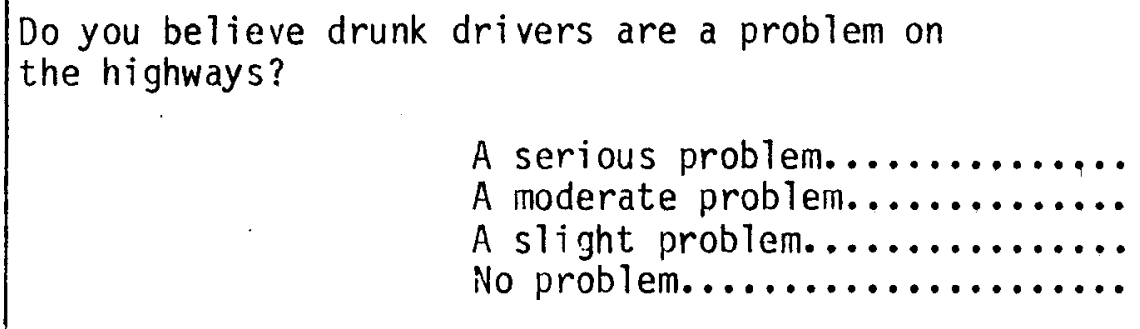 & $\begin{array}{r}67.2 \\
24.8 \\
6.5 \\
1.5\end{array}$ \\
\hline AR & Telephone & 1979 & 403 & 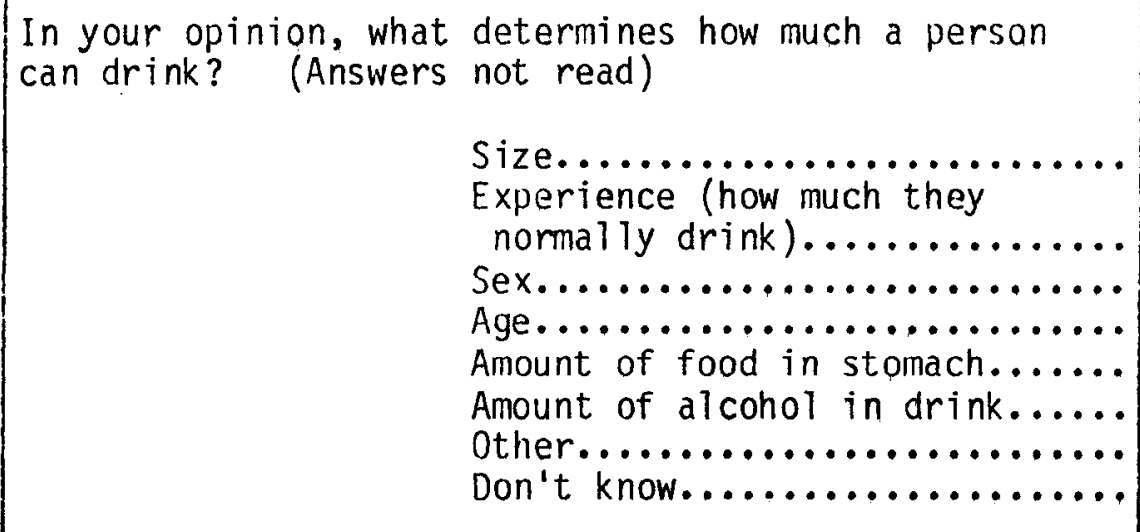 & $\begin{array}{r}24.8 \\
32.8 \\
1.7 \\
3.2 \\
9.4 \\
4.7 \\
16.1 \\
30.8\end{array}$ \\
\hline
\end{tabular}




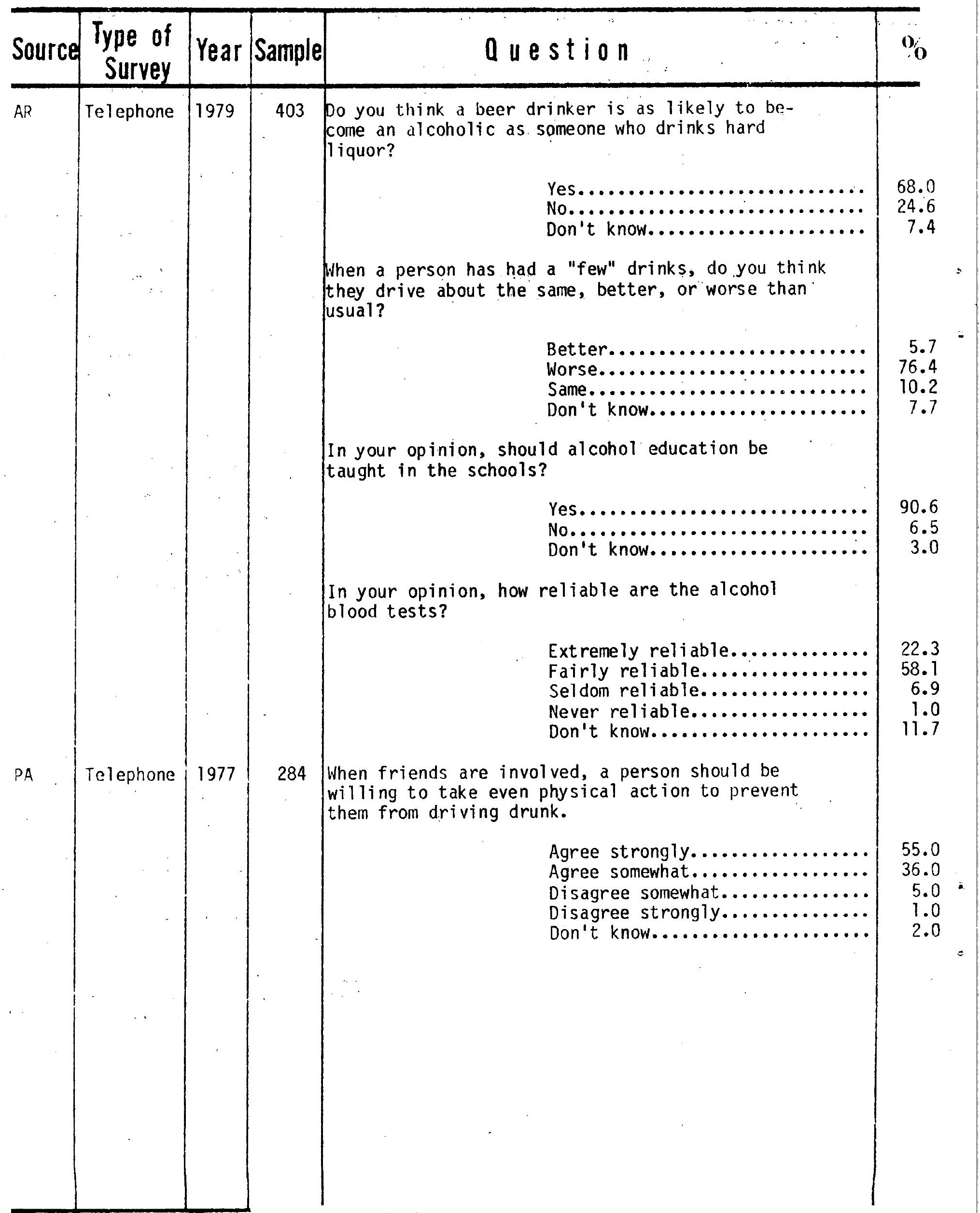




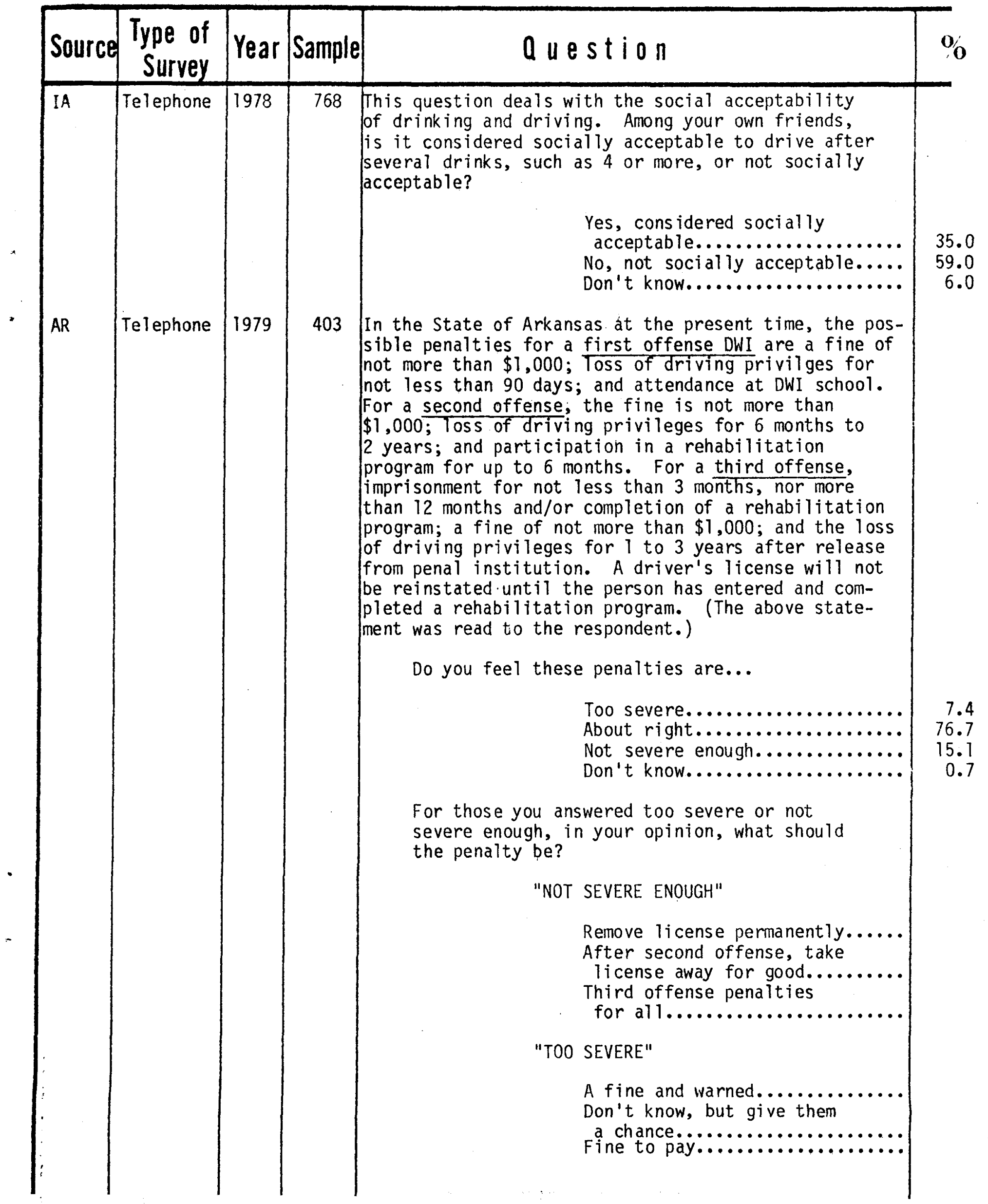




\begin{tabular}{|c|c|c|c|c|c|}
\hline Source & $\begin{array}{l}\text { Type of } \\
\text { Survey }\end{array}$ & Year & Sample & Question & $\%$ \\
\hline iv & Personal & 1977. & 1,669 & 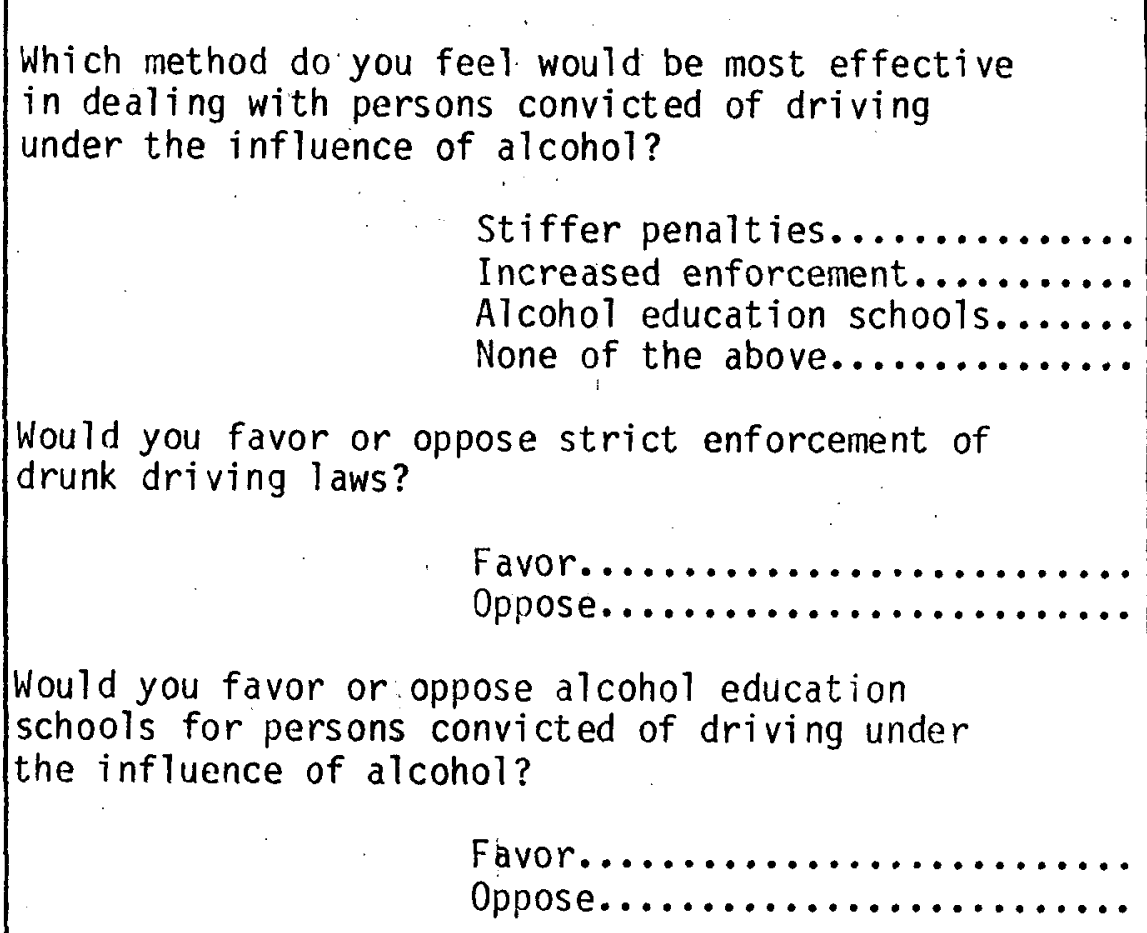 & $\begin{array}{r}52.4 \\
7.3 \\
32.4 \\
7.9\end{array}$ \\
\hline SD & Mail & 1979 & 524 & 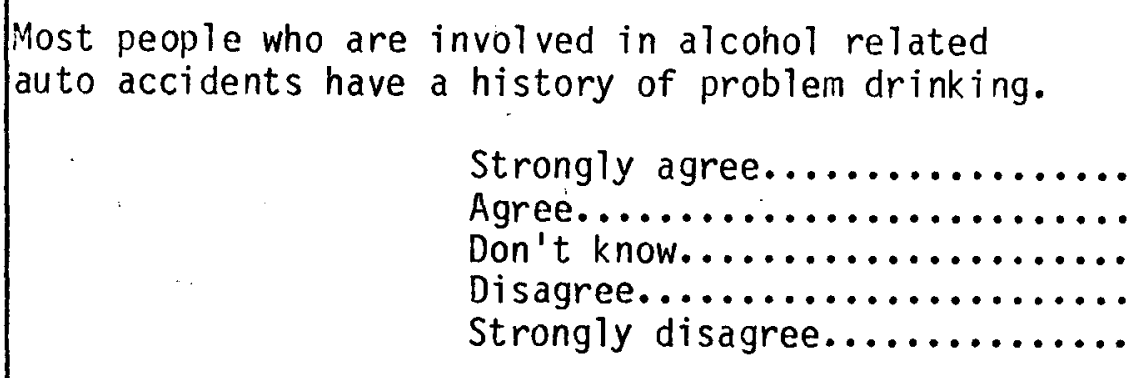 & $\begin{array}{r}8.8 \\
30.9 \\
23.9 \\
30.2 \\
6.2\end{array}$ \\
\hline SD & Mail & 1979 & 524 & 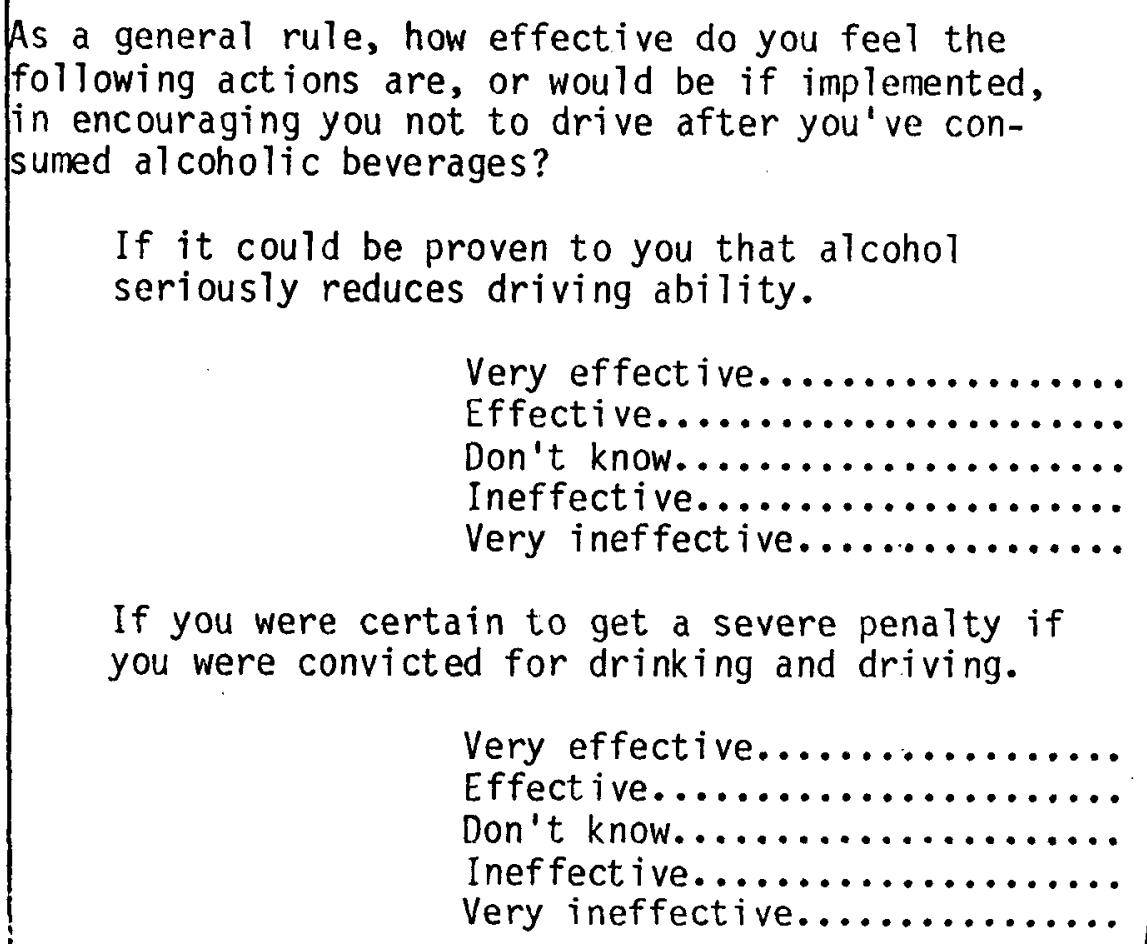 & $\begin{array}{r} \\
37.1 \\
43.3 \\
11.2 \\
6.3 \\
2.2\end{array}$ \\
\hline
\end{tabular}




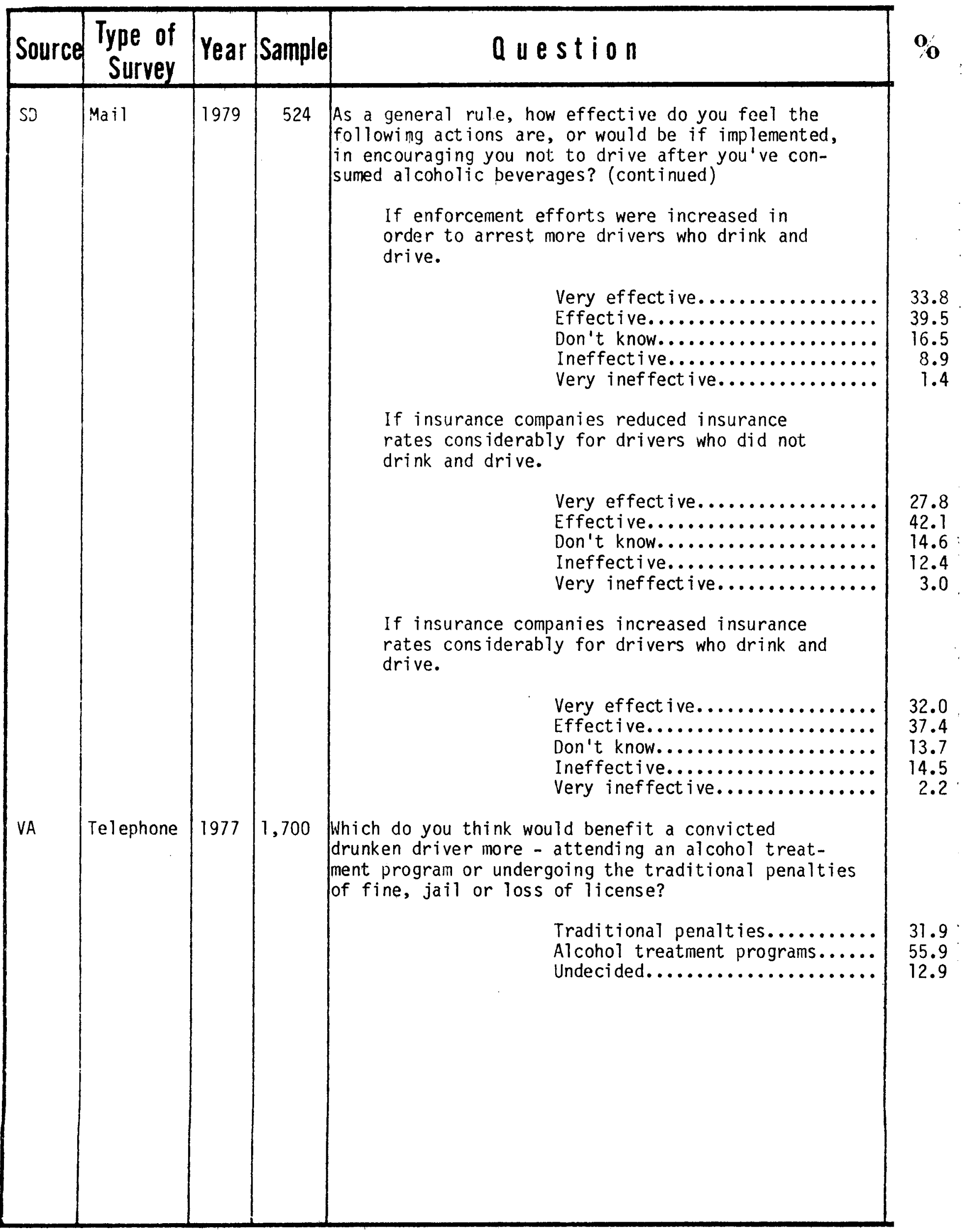




\section{BEHAVIOR}

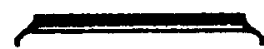




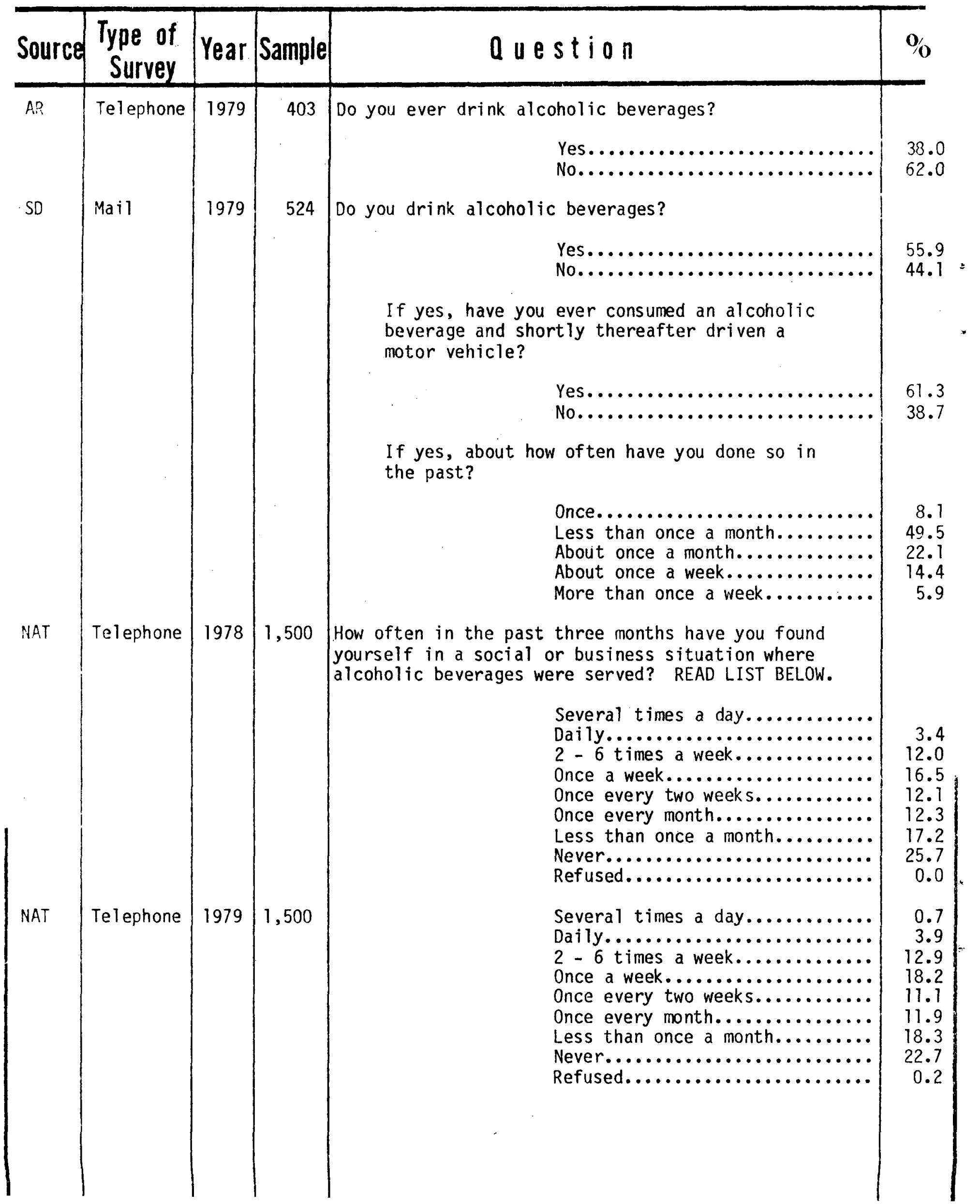




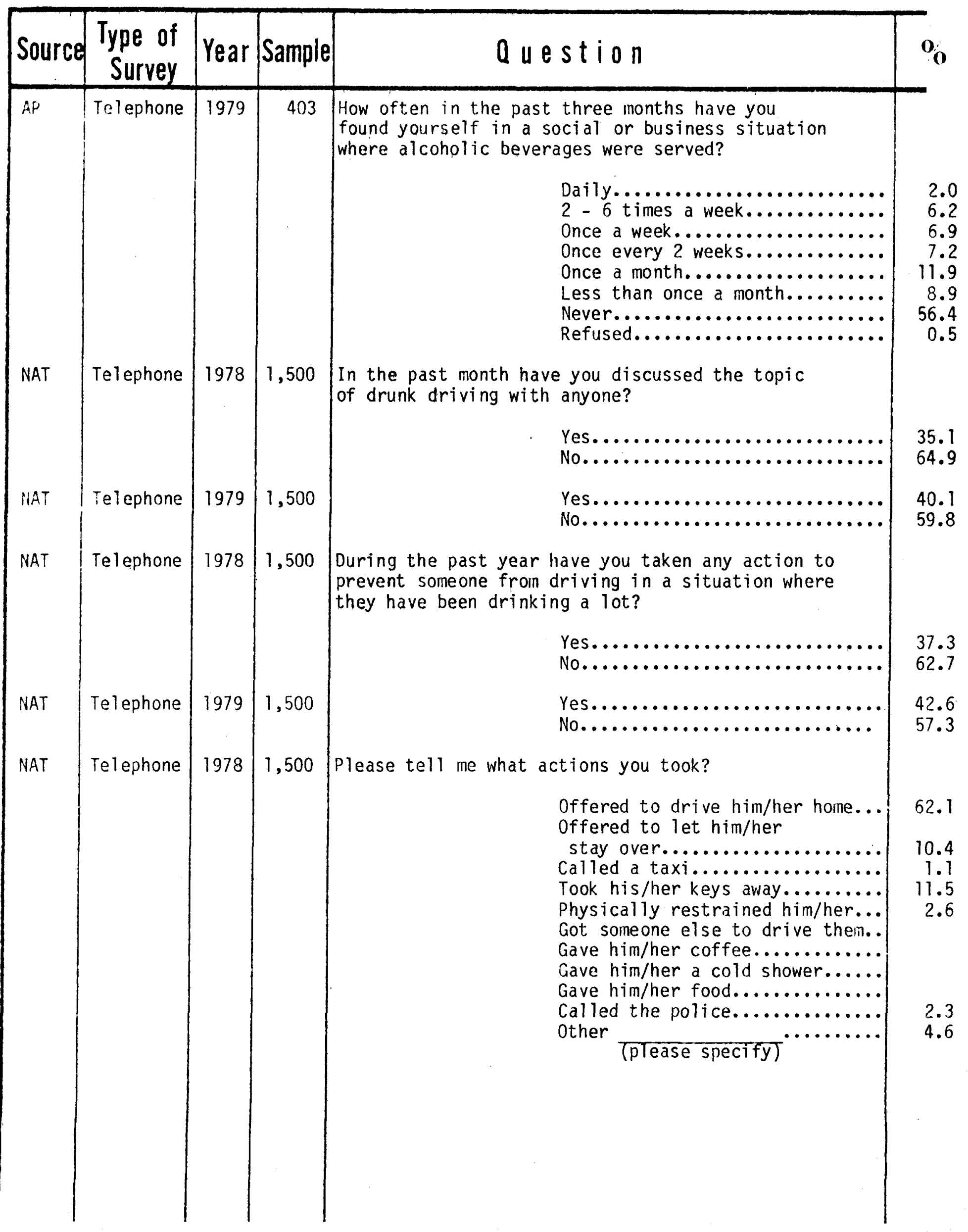




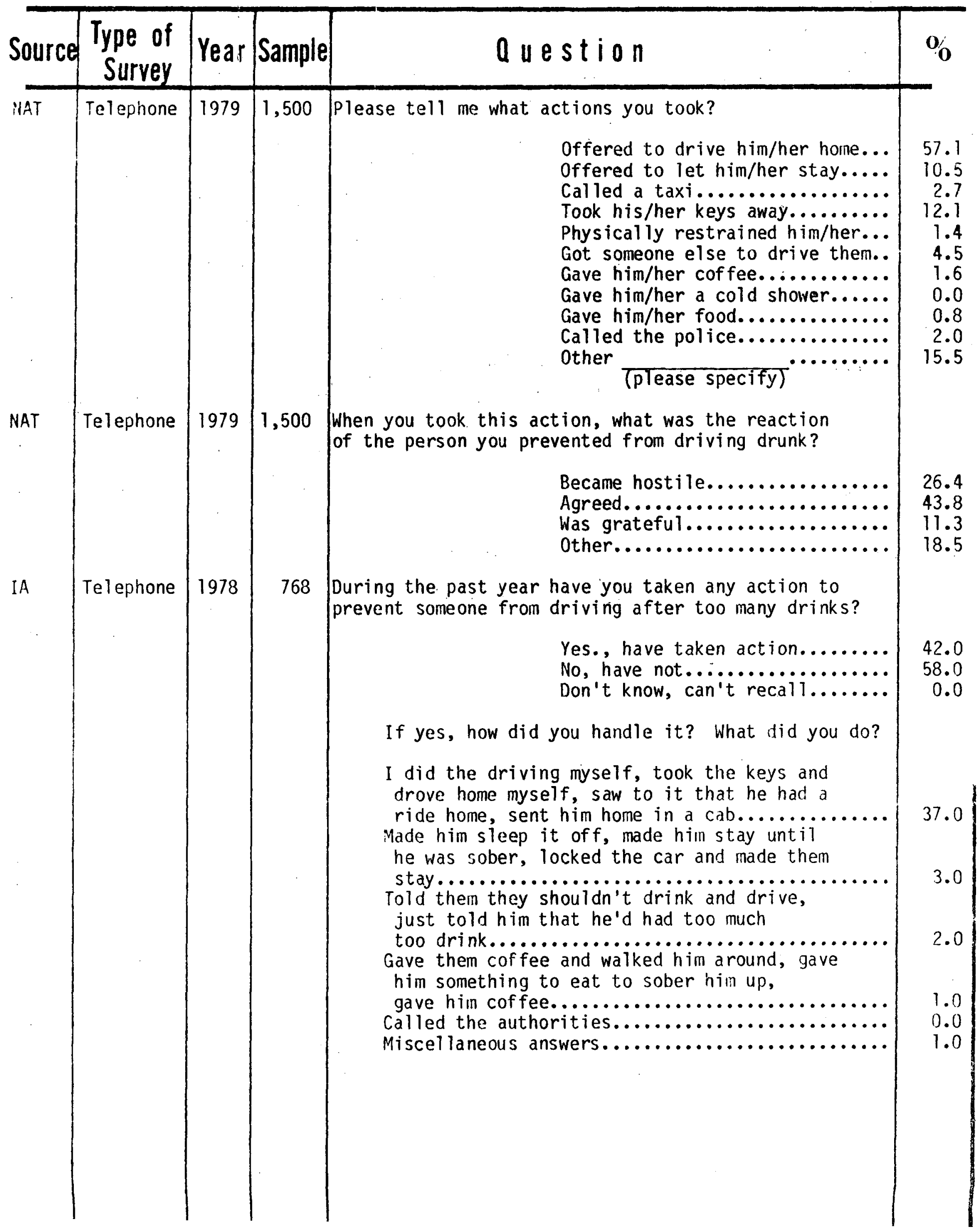




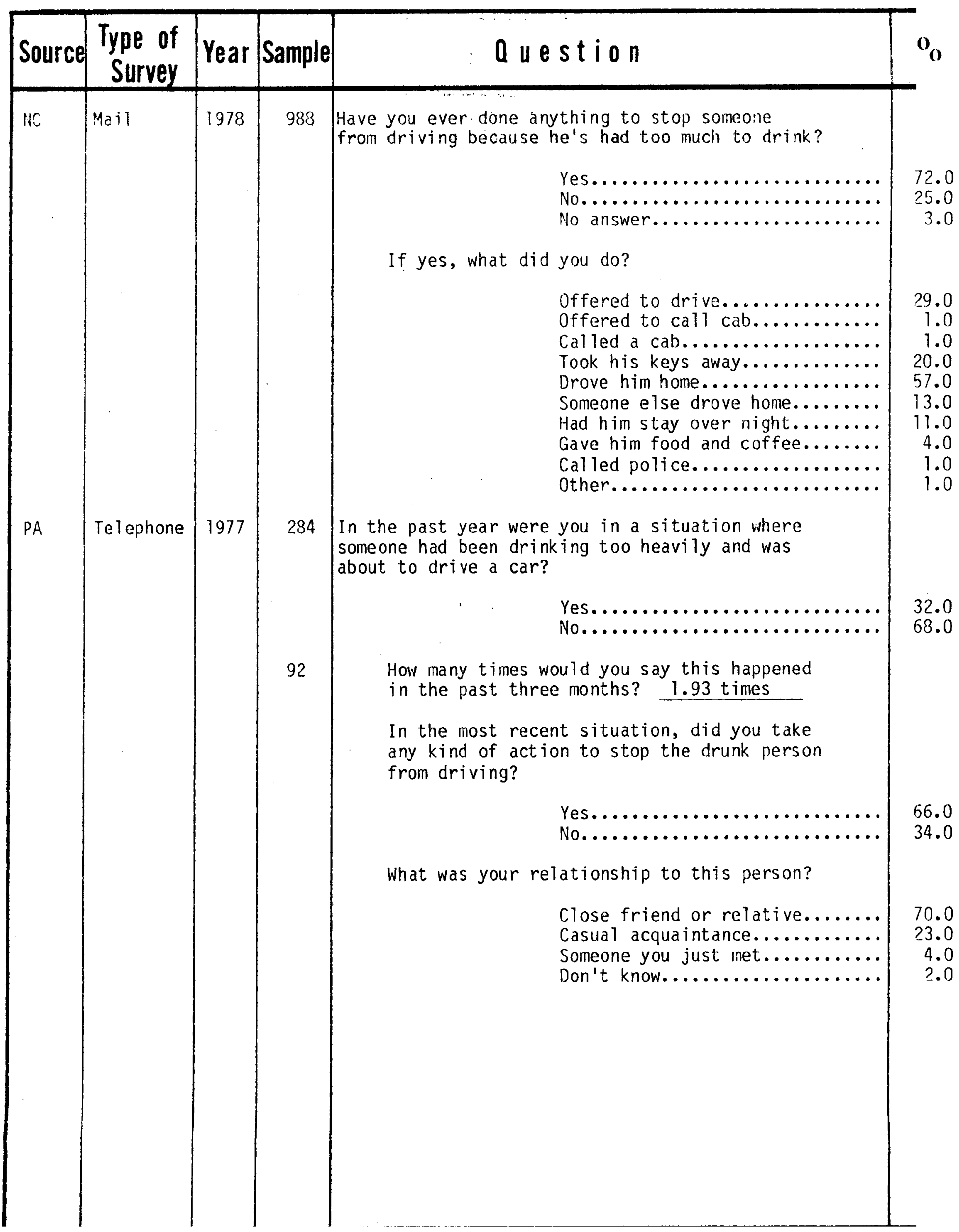




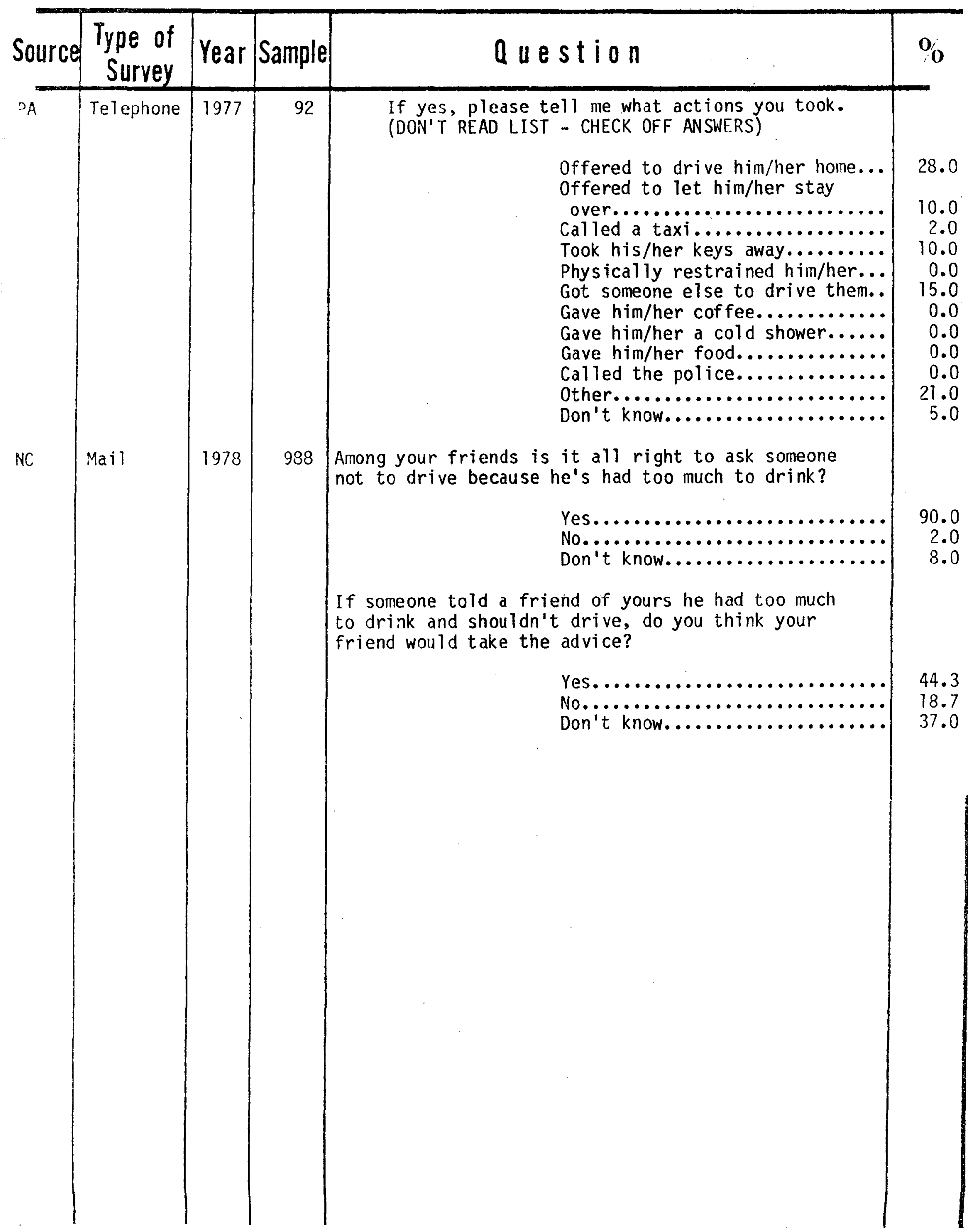


KNOWLEDGE $\longrightarrow$ 


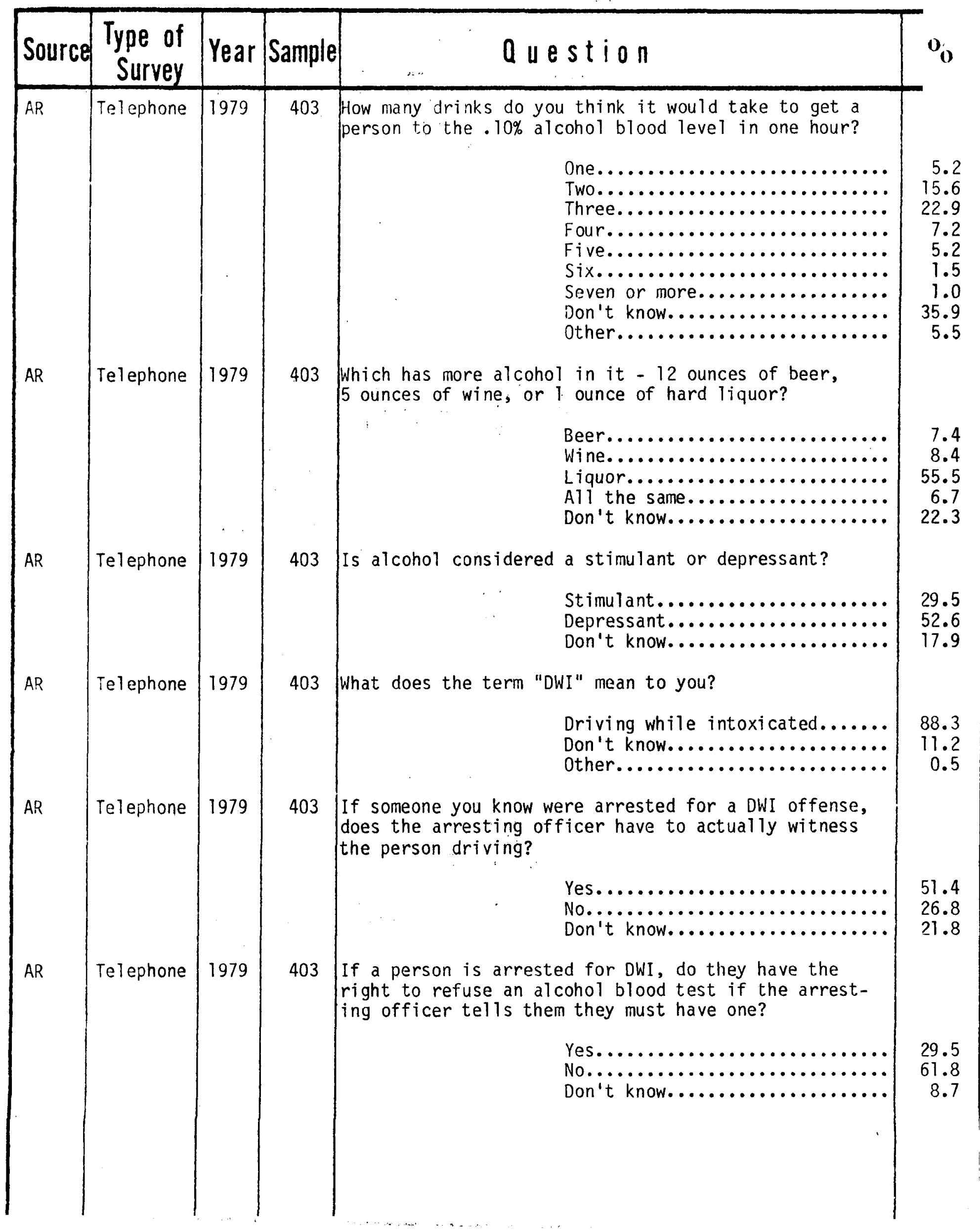




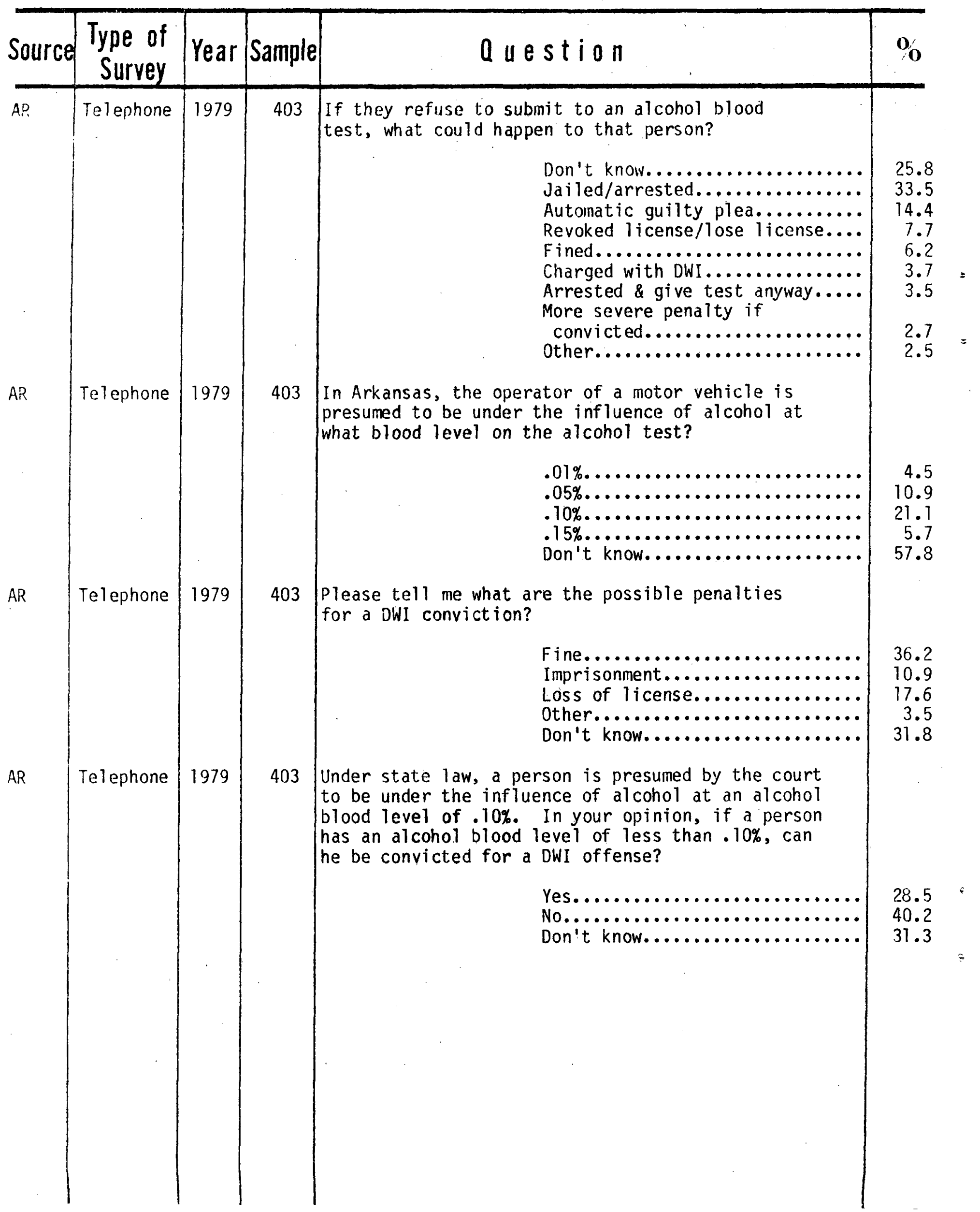




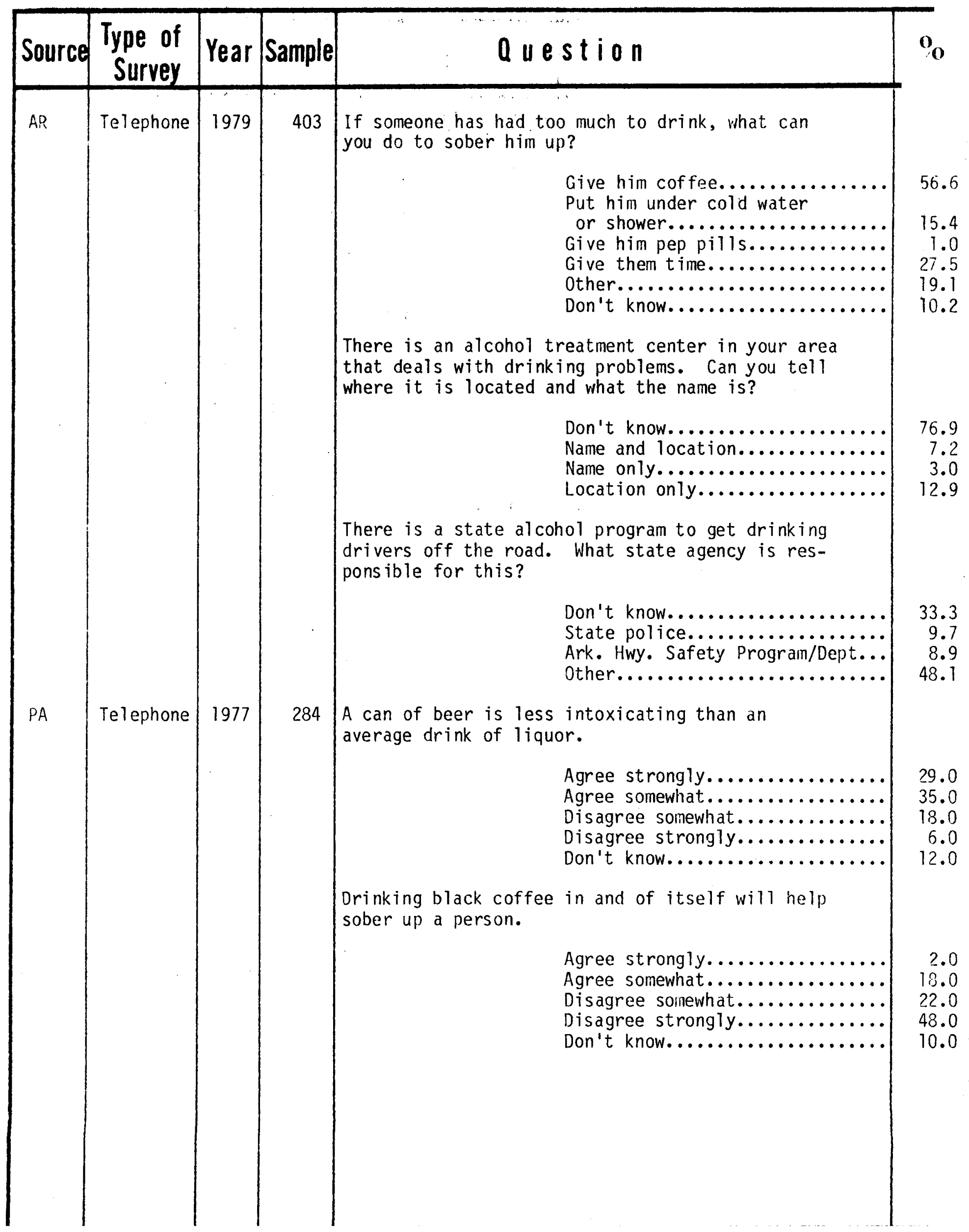




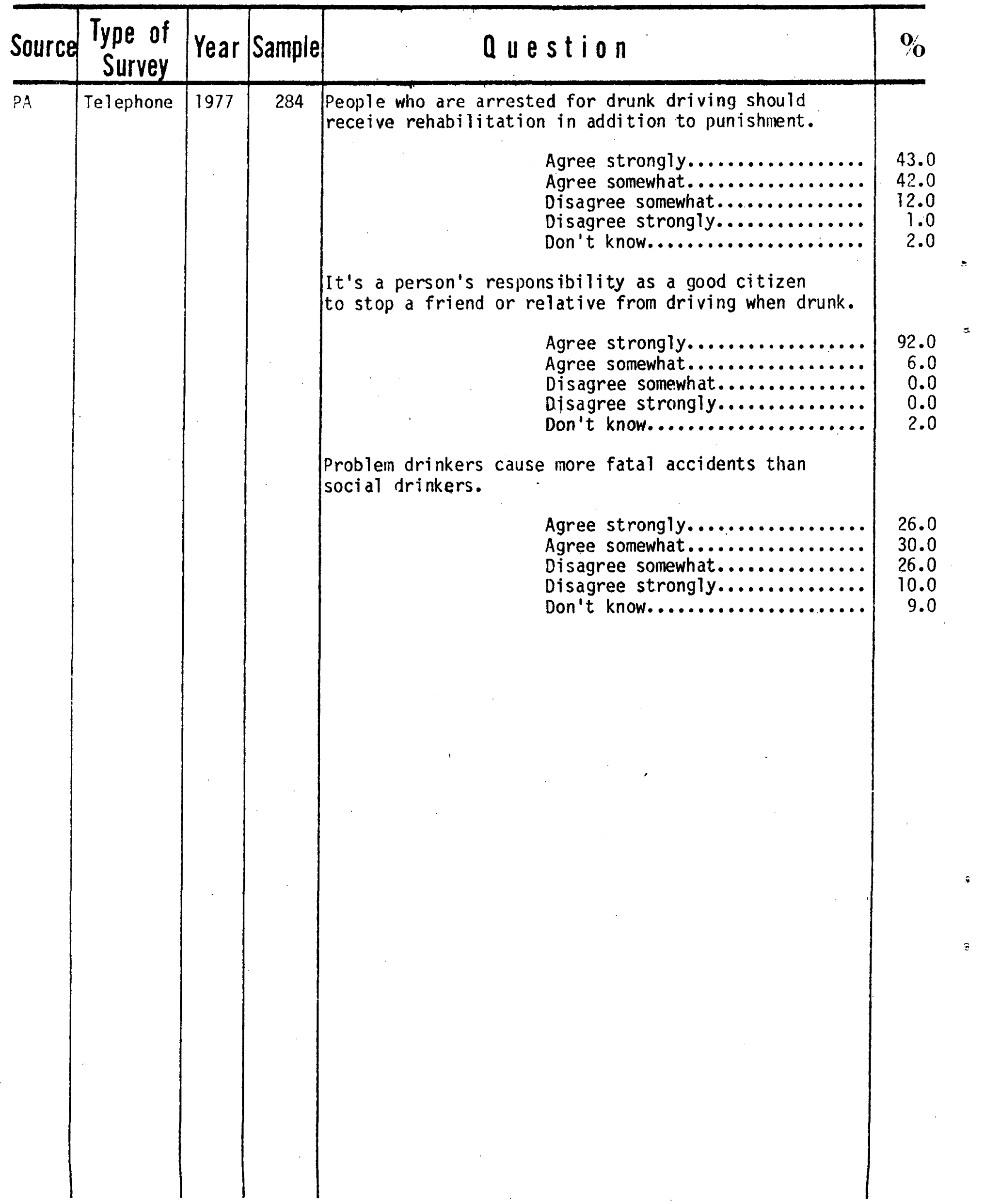




\section{PUBLIC INFORMATION AN D \\ EDUCATION}

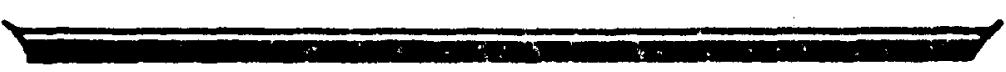


ATTITUDE

$\longrightarrow$ 
PI\&E-A- ]

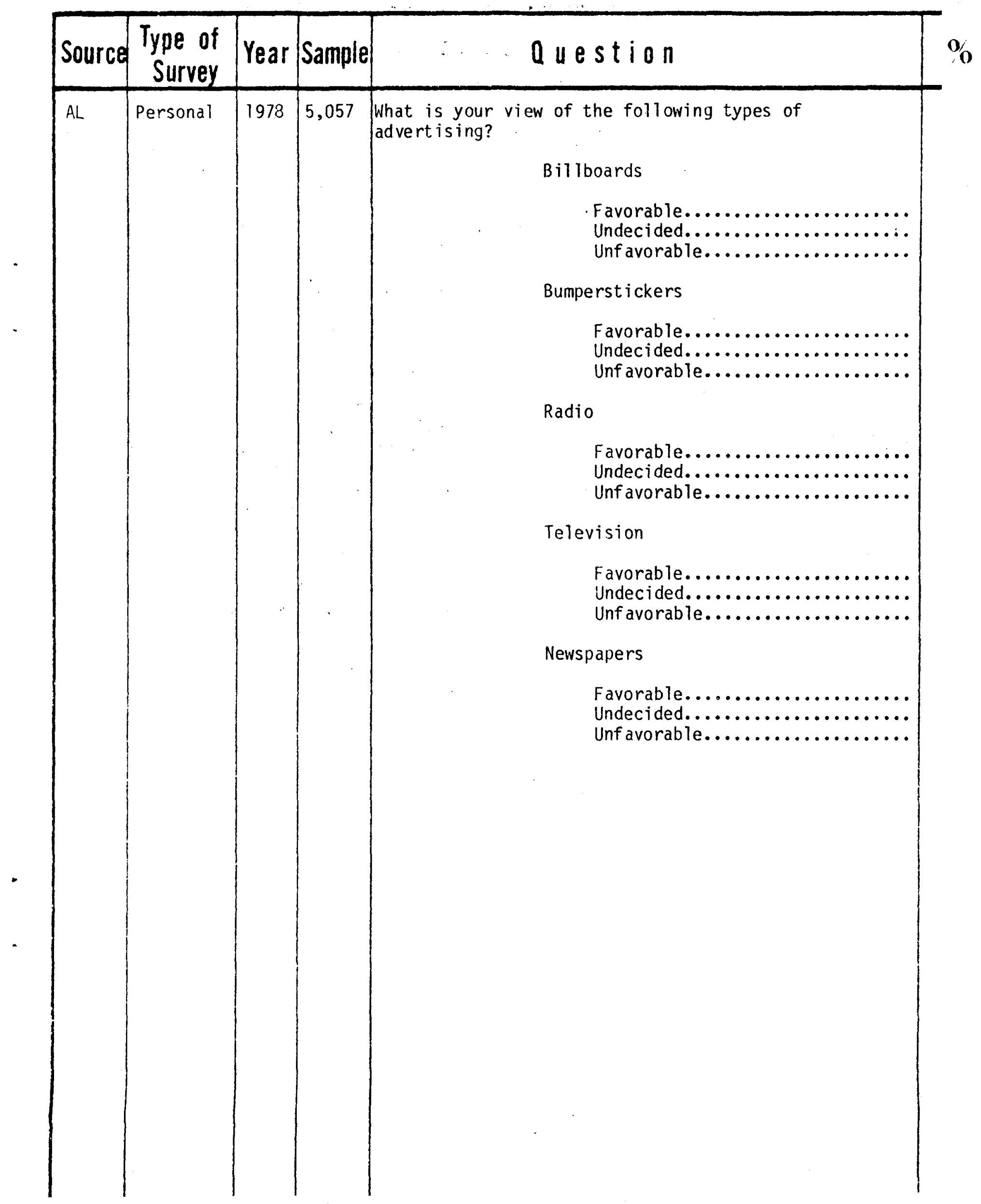




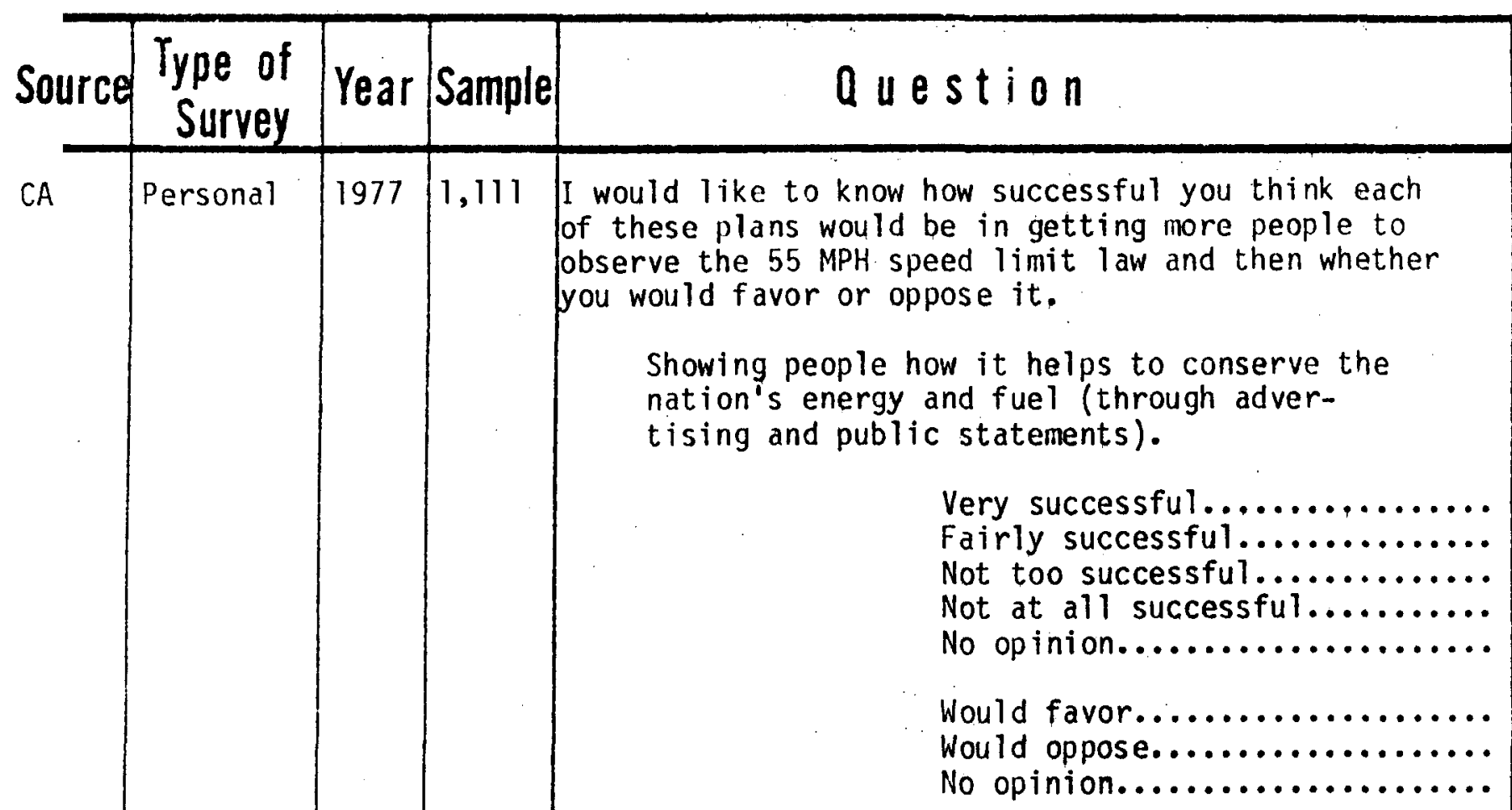

Showing people how it helps to save lives

(through advertising and public statements).

Very successful................

Fairly successful...............

Not too successful...............

Not at all successfur............

No opinion....................

Would favor...

Would oppose...................

No opinion...................

28.0

50.0

16.0

5.0

2.0

87.0

10.0 


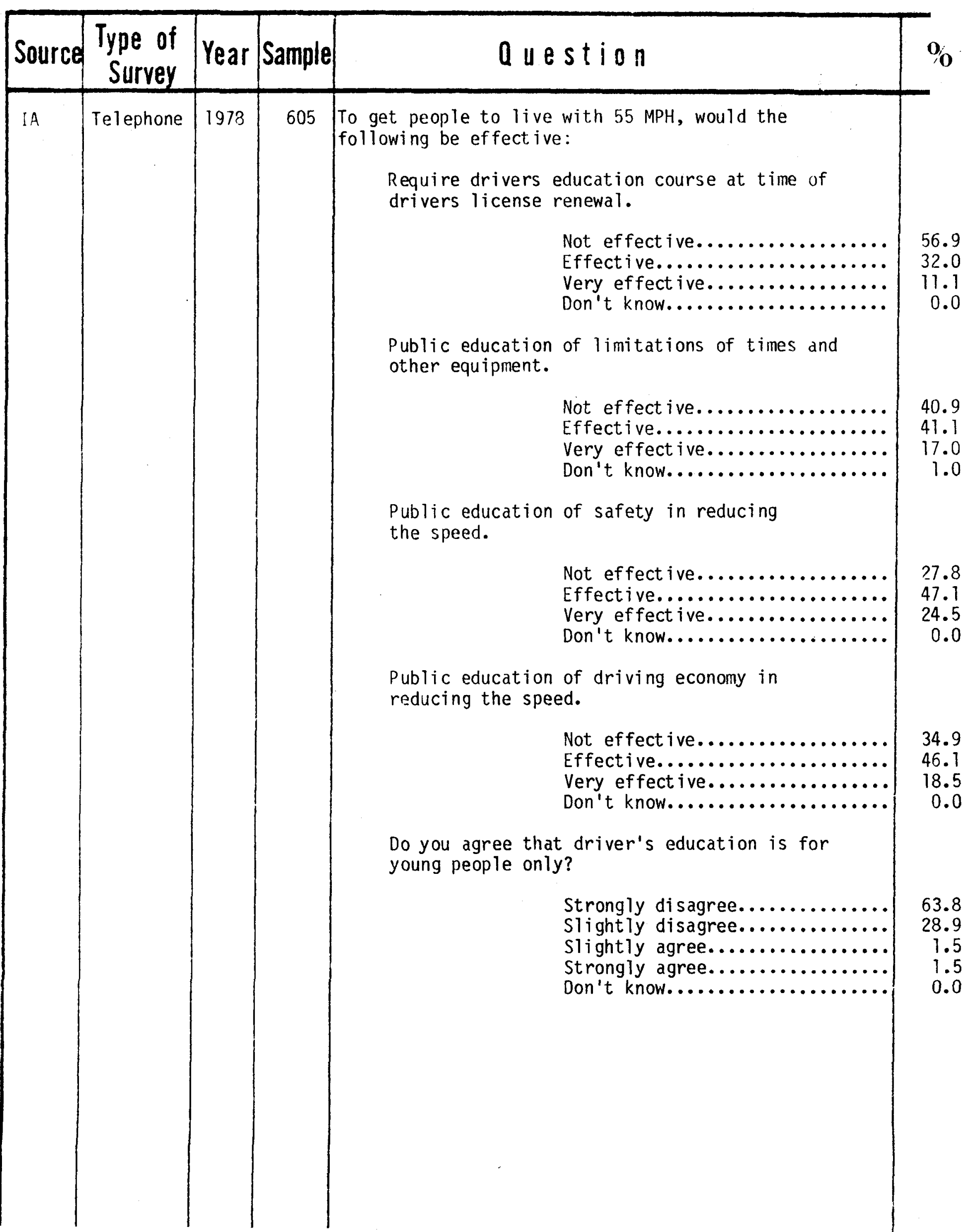


KNOWLEDGE

$$
\longrightarrow
$$




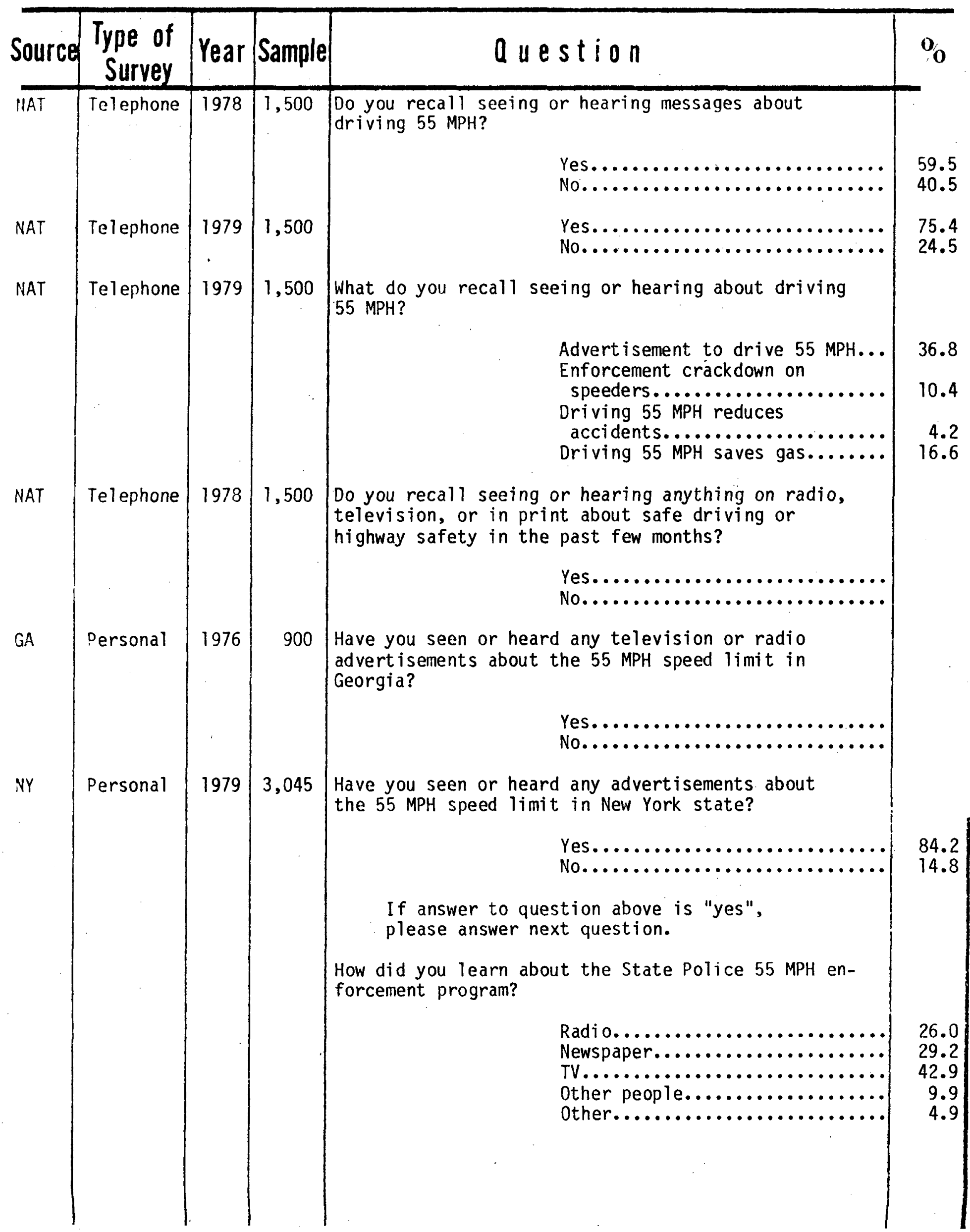




\begin{tabular}{|c|c|c|c|c|}
\hline Source & $\begin{array}{l}\text { Type of } \\
\text { Survey }\end{array}$ & Year & Sample & auestion \\
\hline$P A$ & Telephone & 1979 & 500 & 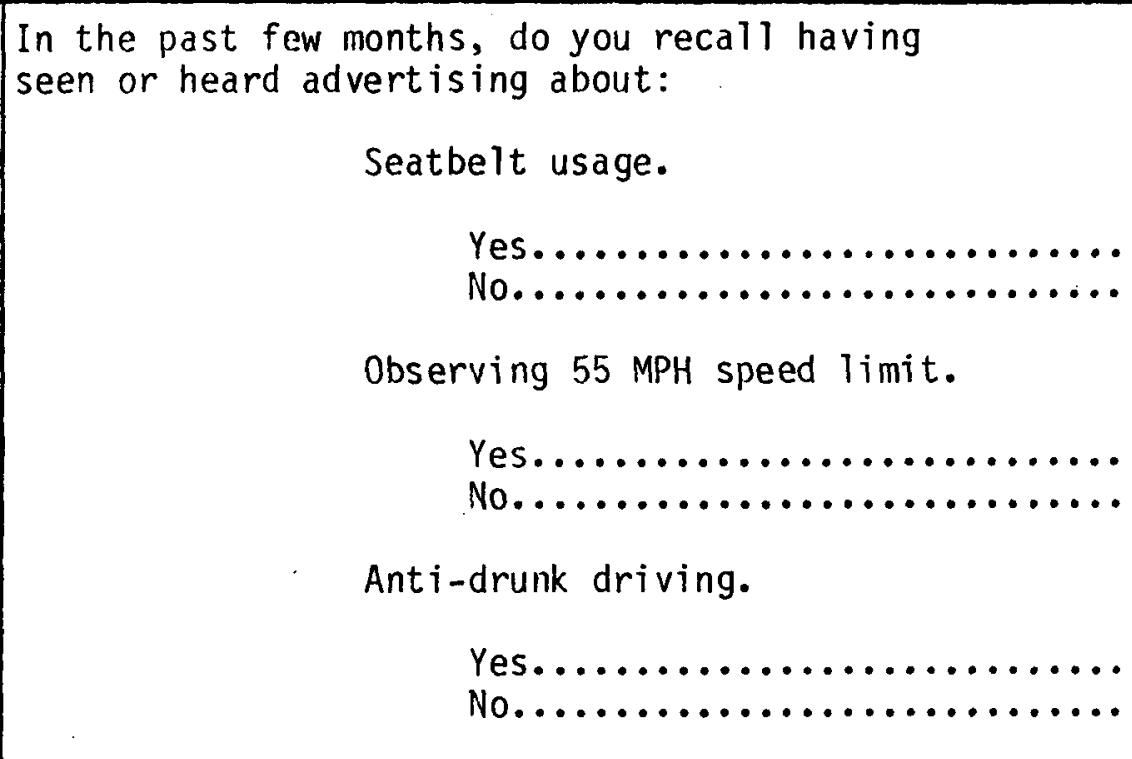 \\
\hline PA & Telephone & 1977 & 500 & 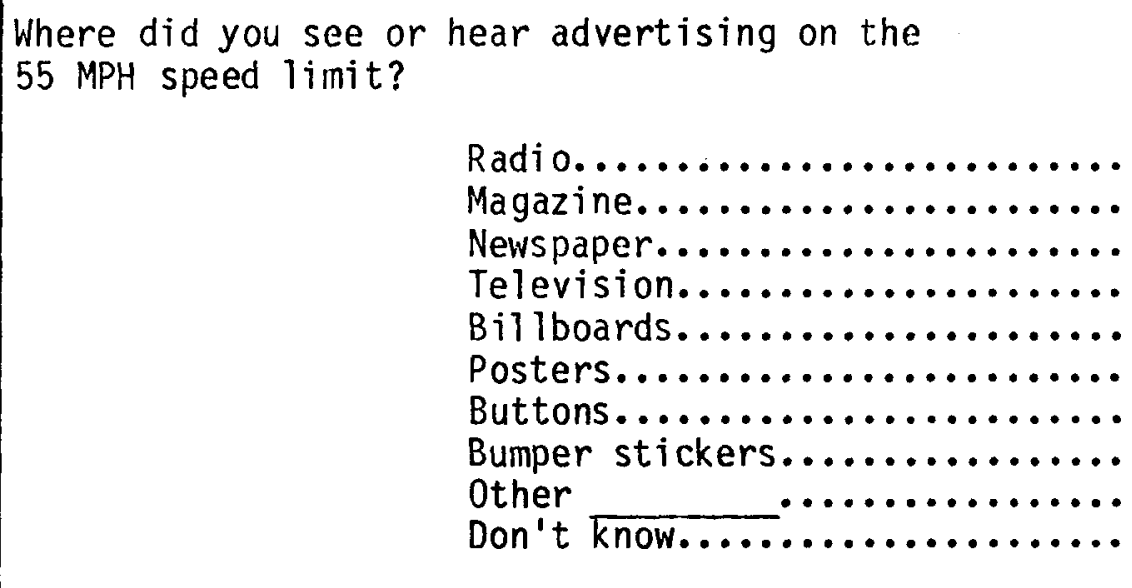 \\
\hline WI & Personal & 1978 & 6,565 & 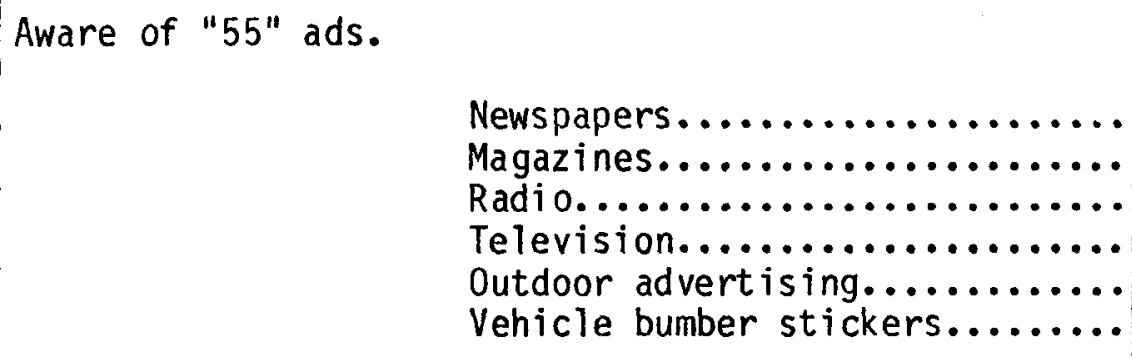 \\
\hline
\end{tabular}




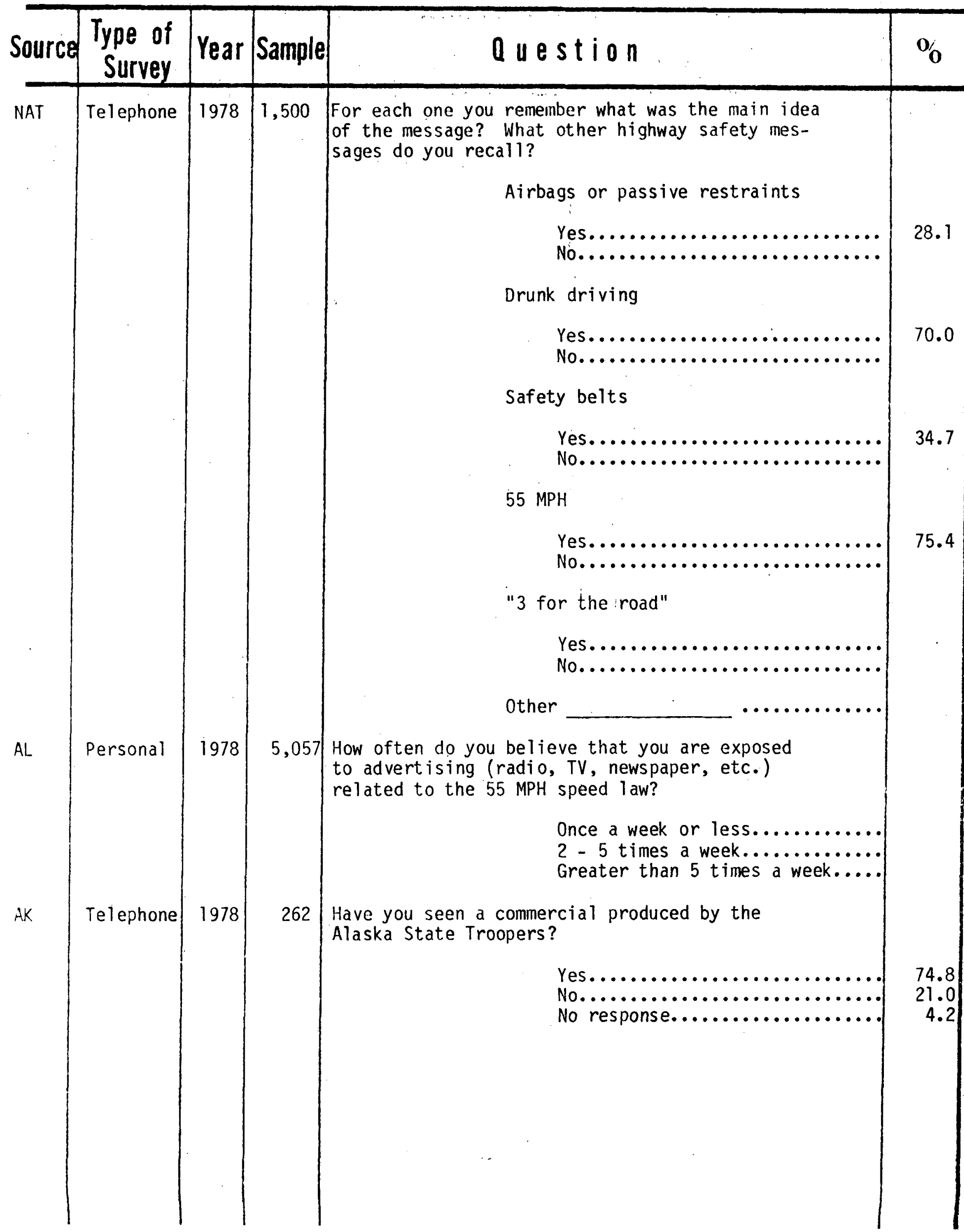




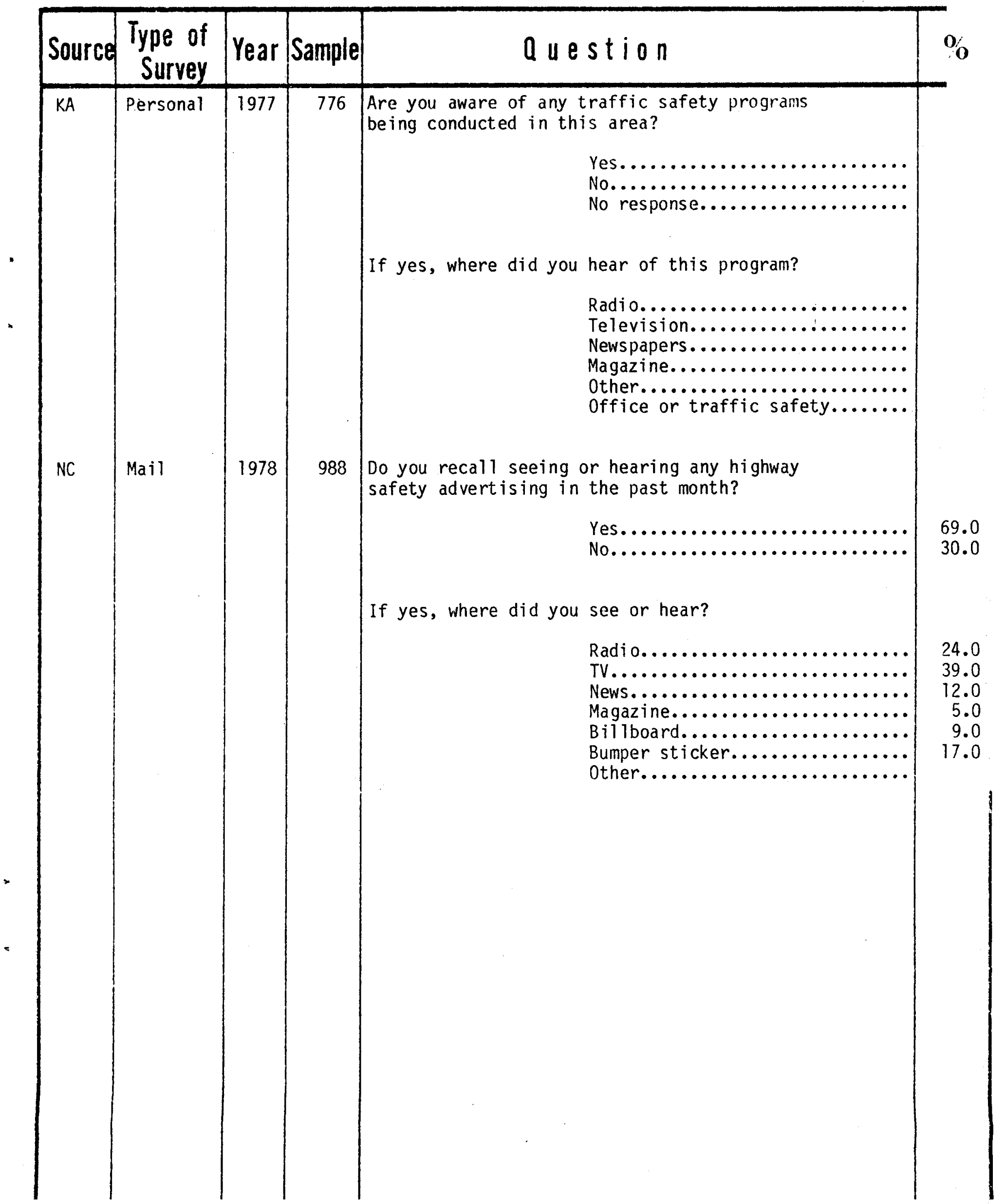




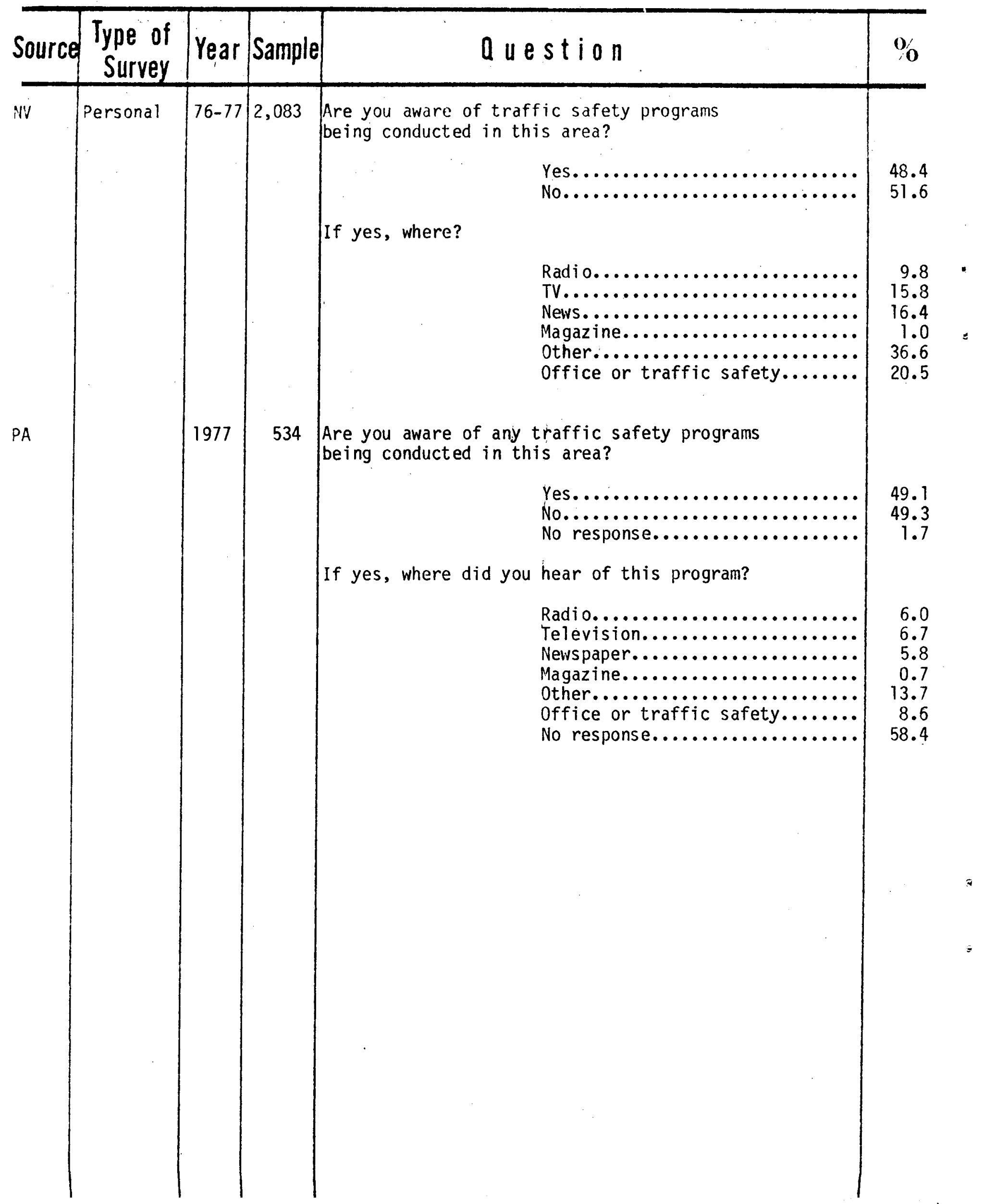




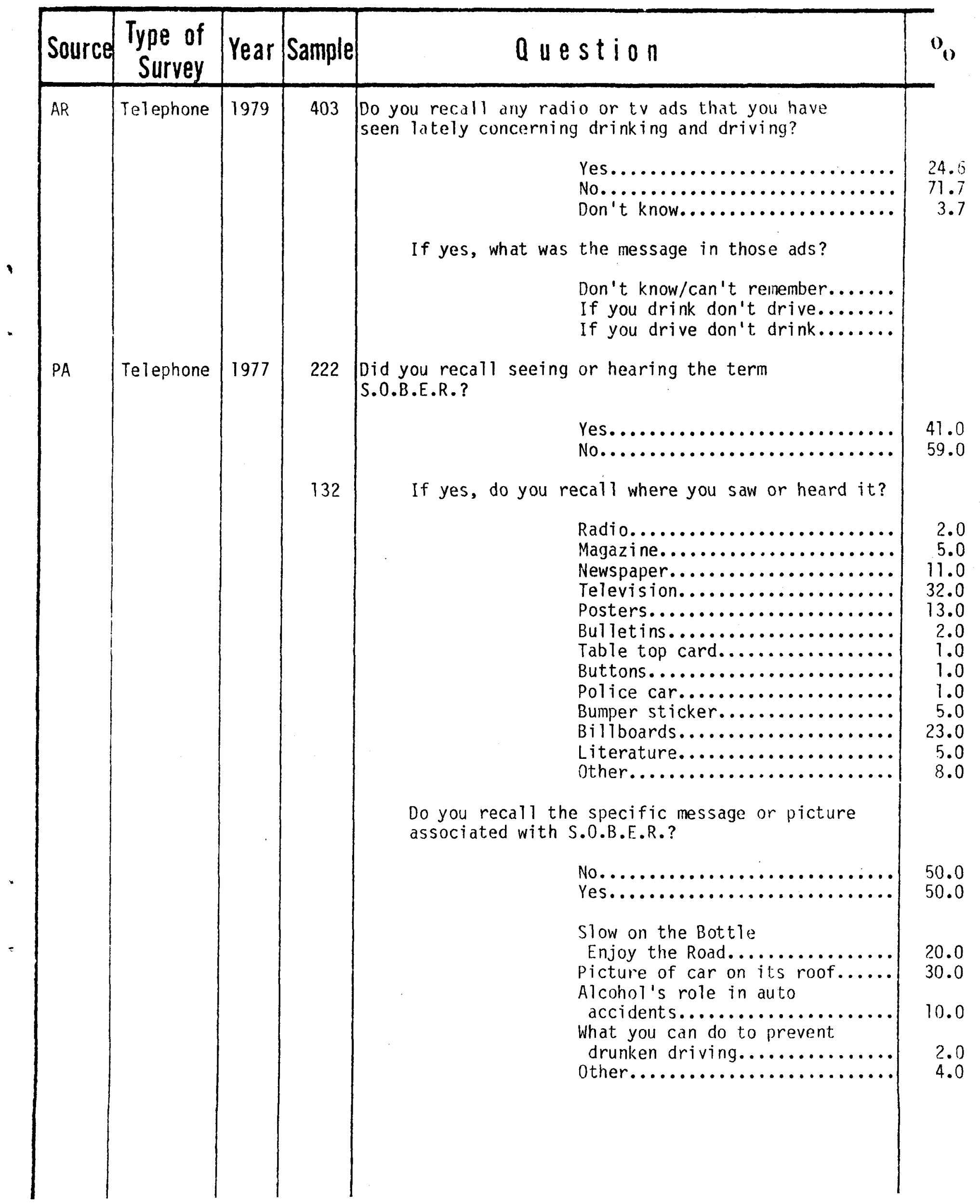




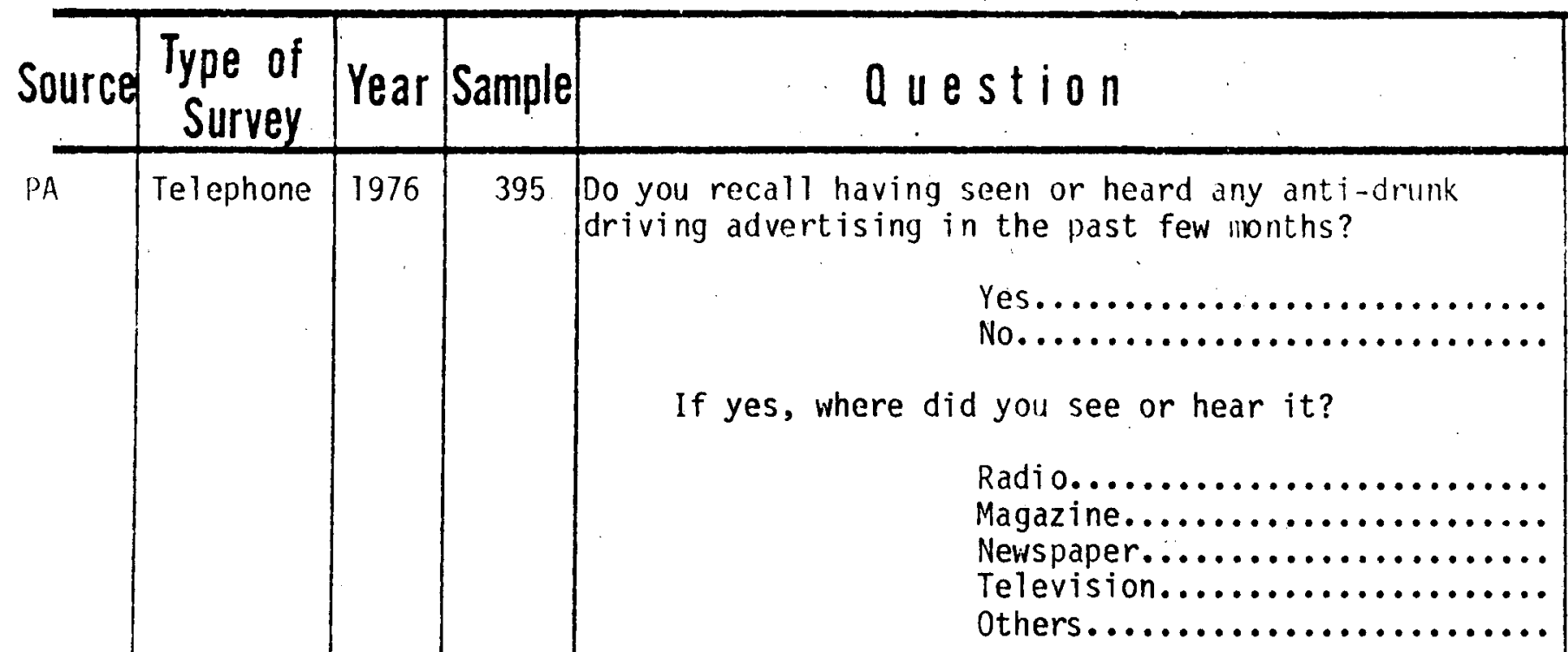

$0_{0}$

In your own words, what did the advertising say?

Relating to taking preventive measure......................

Relating to self action..........

Non-involvement of self.........

42.0

29.0

29.0

Though you may have mentioned it before, do you remember seeing an ad where:

A man in his bathrobe tells how he saved his friend's life by having him stay over instead of driving drunk.

Yes........................

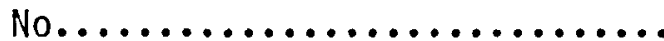

Don't know..................

4.0

95.0

1.0

A bartender describes sending an intoxicated patron home in a taxi.

Yes.......................

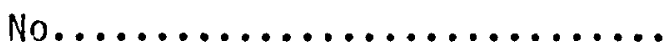

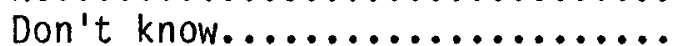


PI\&E-K- 8

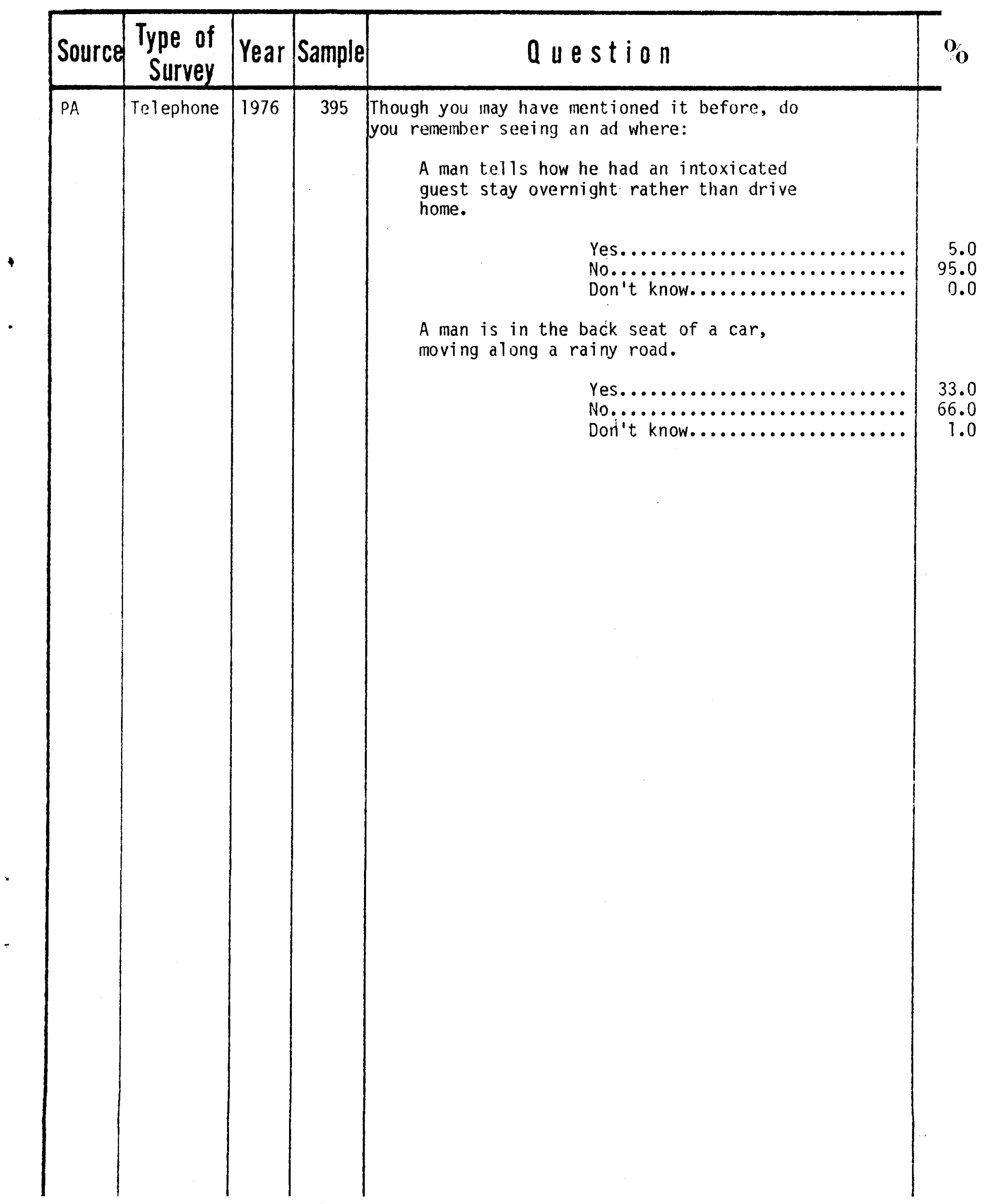




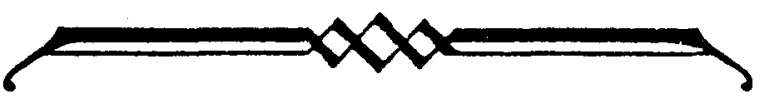
SAFETY HELMETS 
ATTITUDE

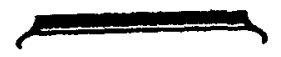




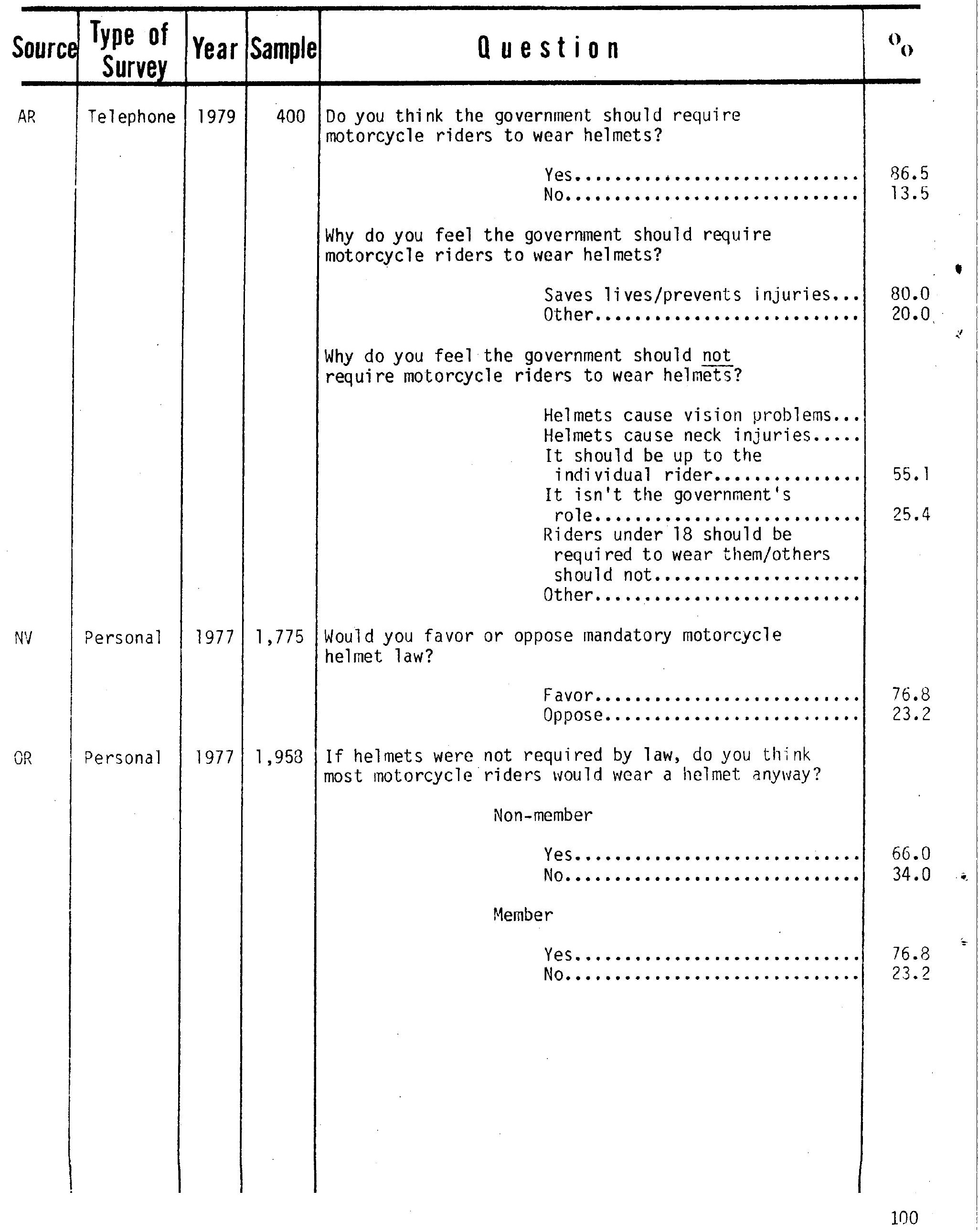


SH-A-2

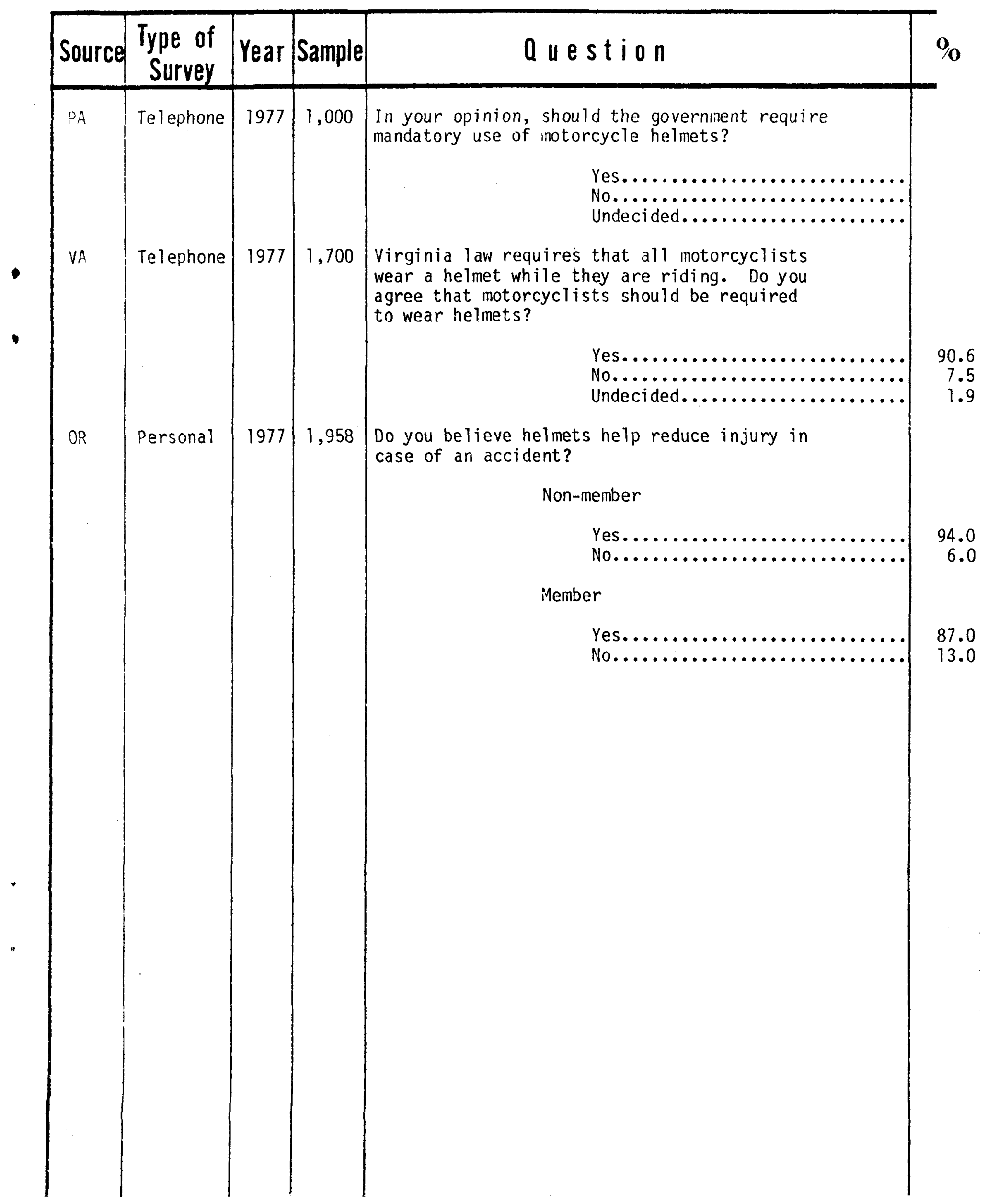




\section{BEHAVIOR

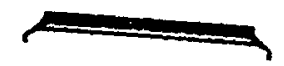




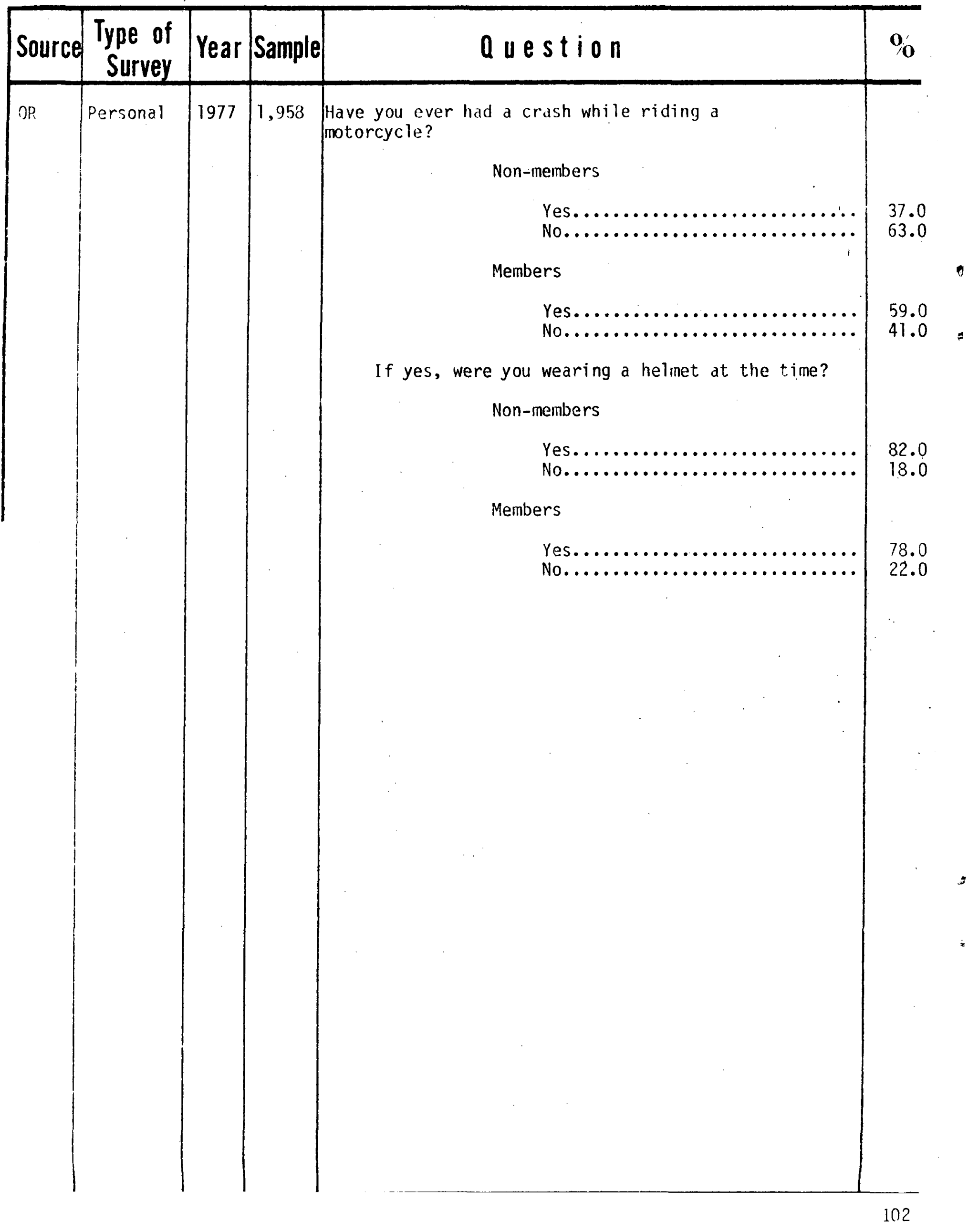


KNOWLEDGE

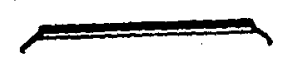




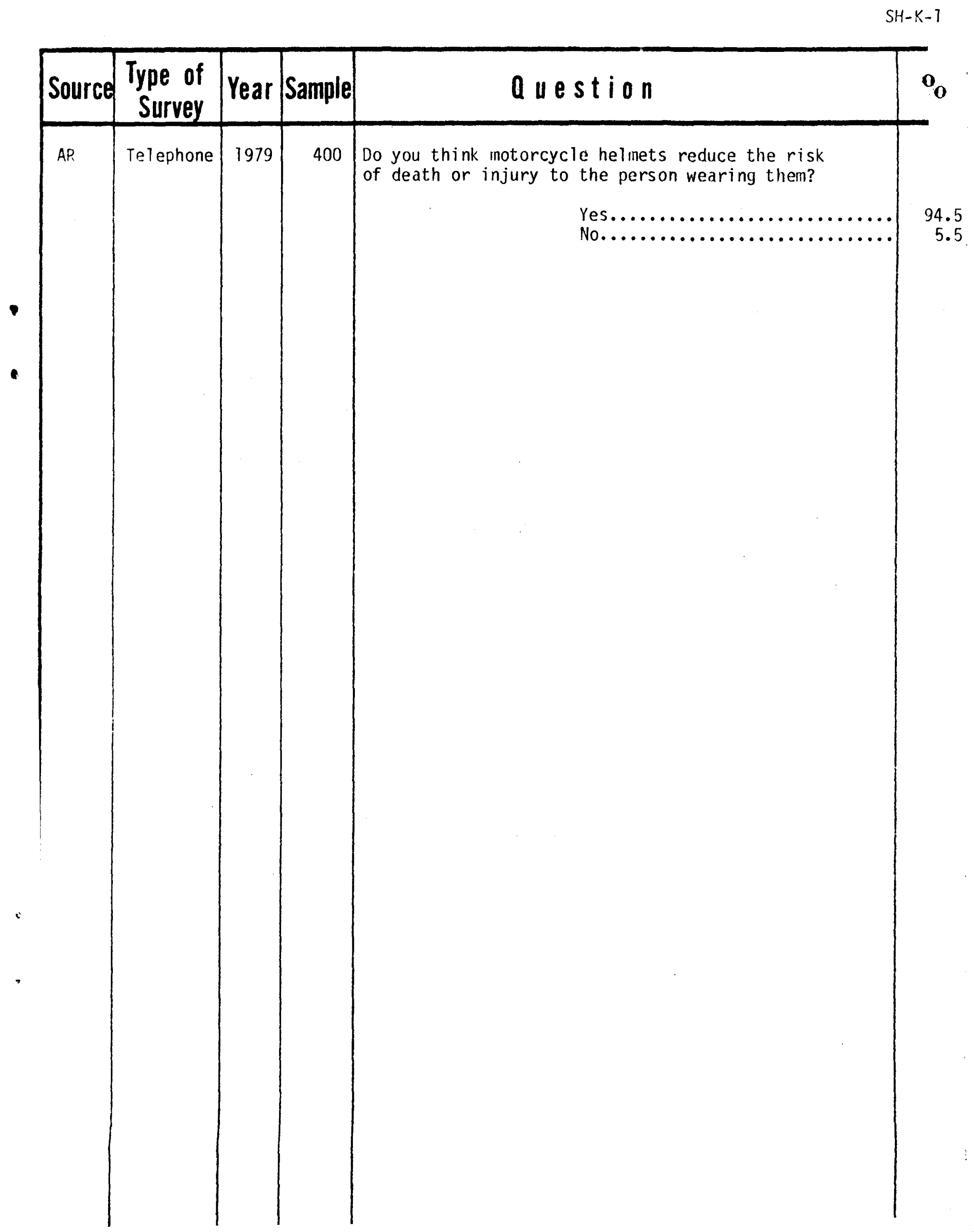




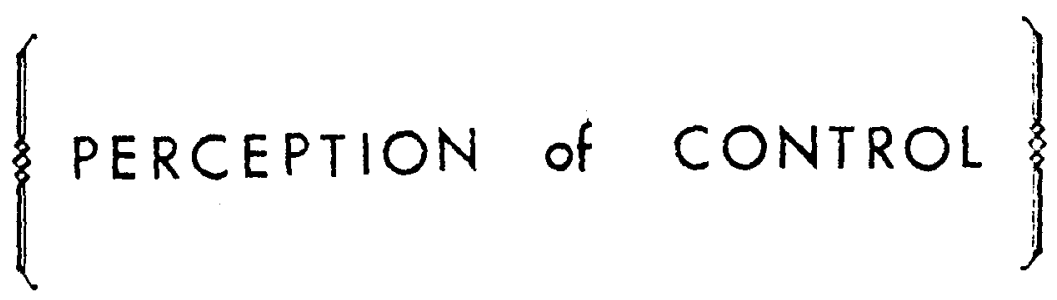




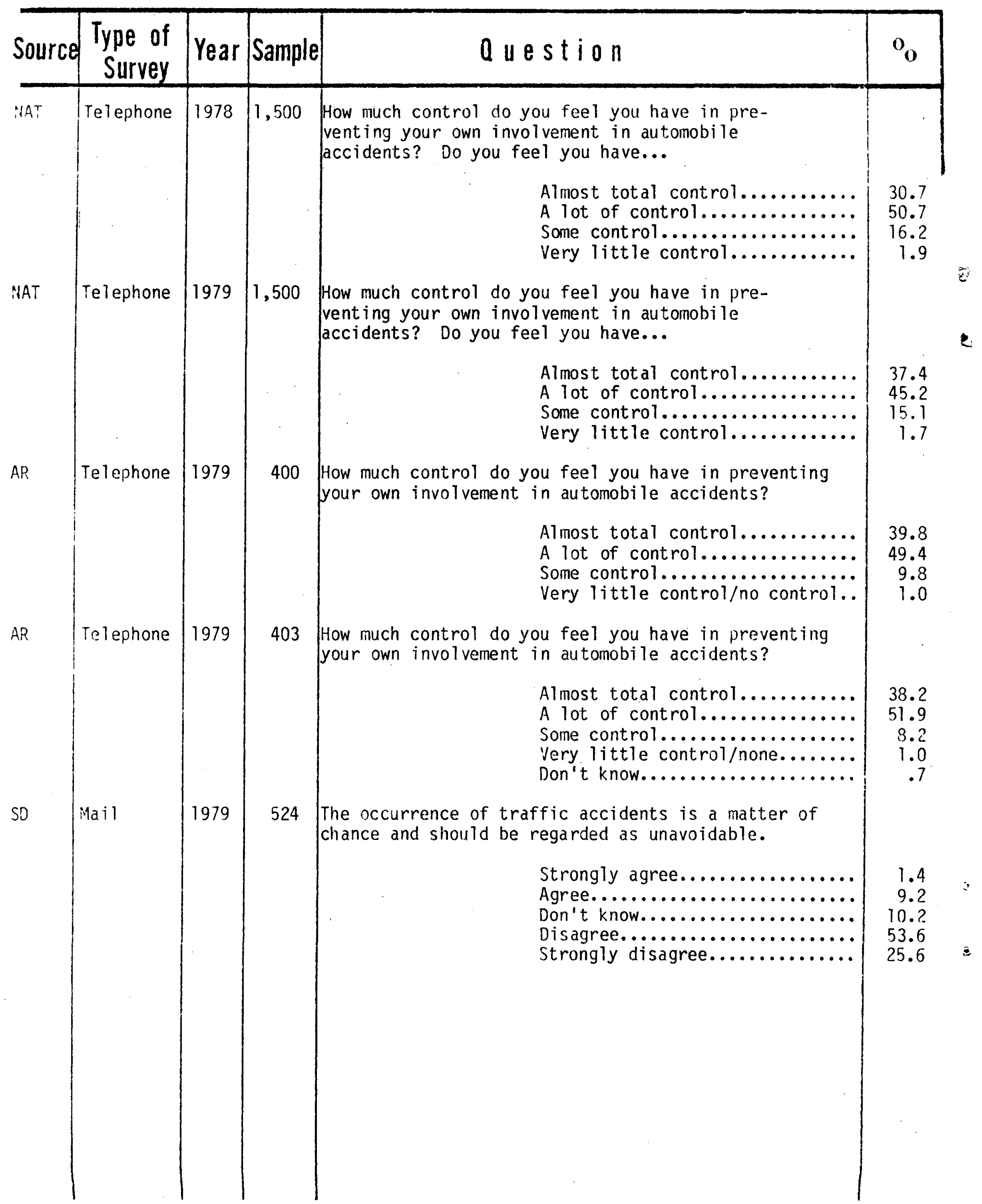




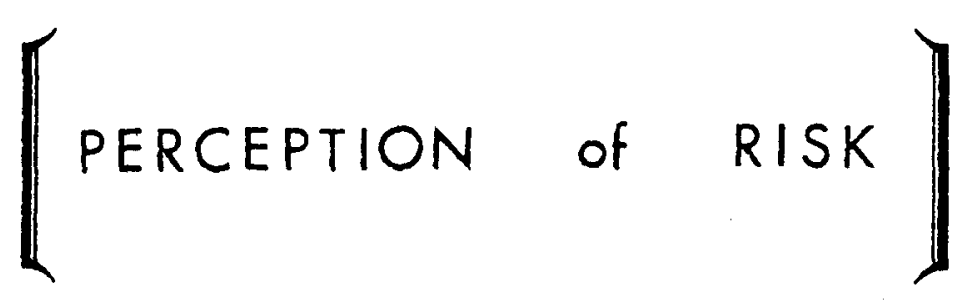




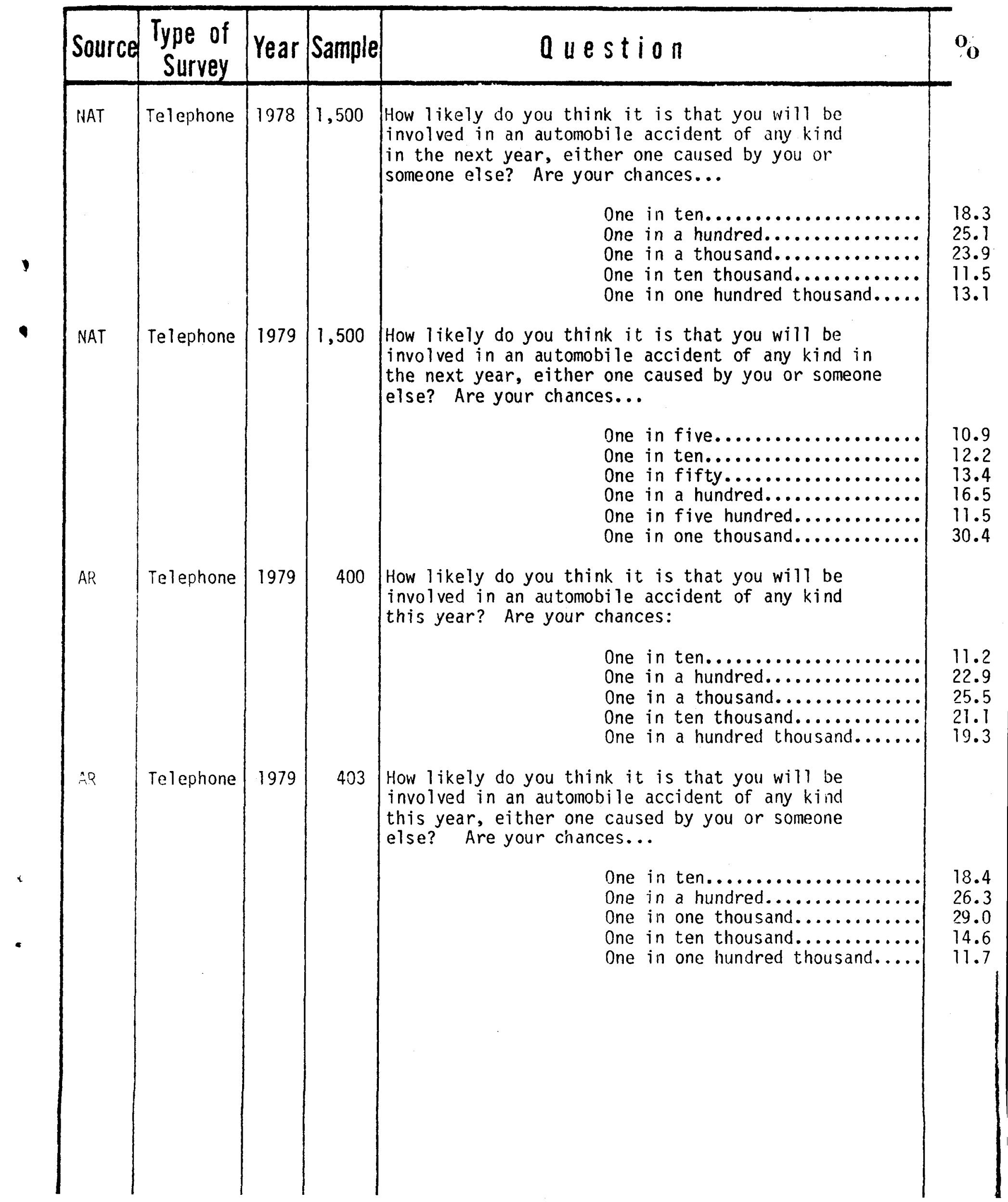




\section{DEMOGRAPHIC $\longrightarrow x$}




\begin{tabular}{|c|c|c|c|c|c|}
\hline Source & $\begin{array}{l}\text { Type of } \\
\text { Survey }\end{array}$ & Year & Sample & Question & 0 \\
\hline NAT & Telephone & 1978 & 1,500 & 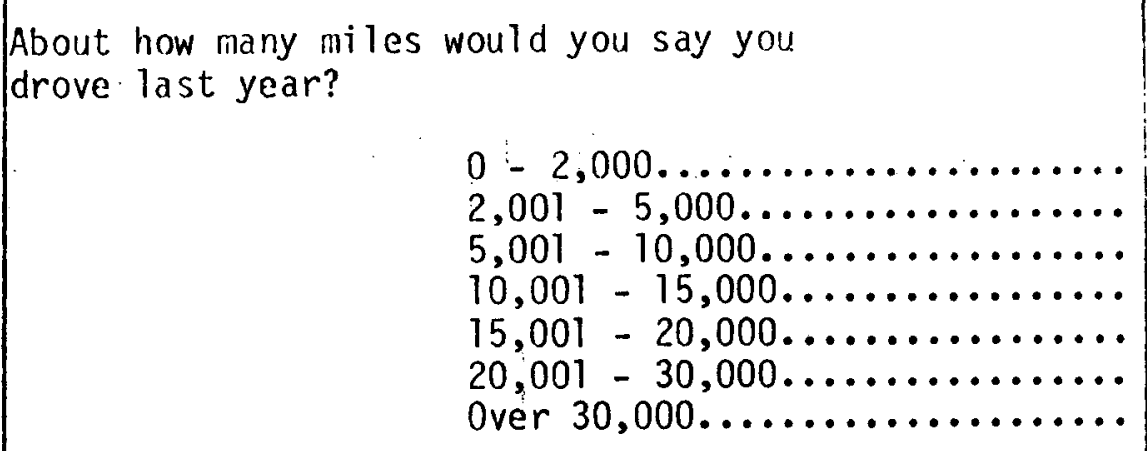 & $\begin{array}{r}15.0 \\
11.0 \\
20.0 \\
18.0 \\
11.0 \\
9.0 \\
7.0\end{array}$ \\
\hline NAT & Telephone & 1979 & 1,500 & 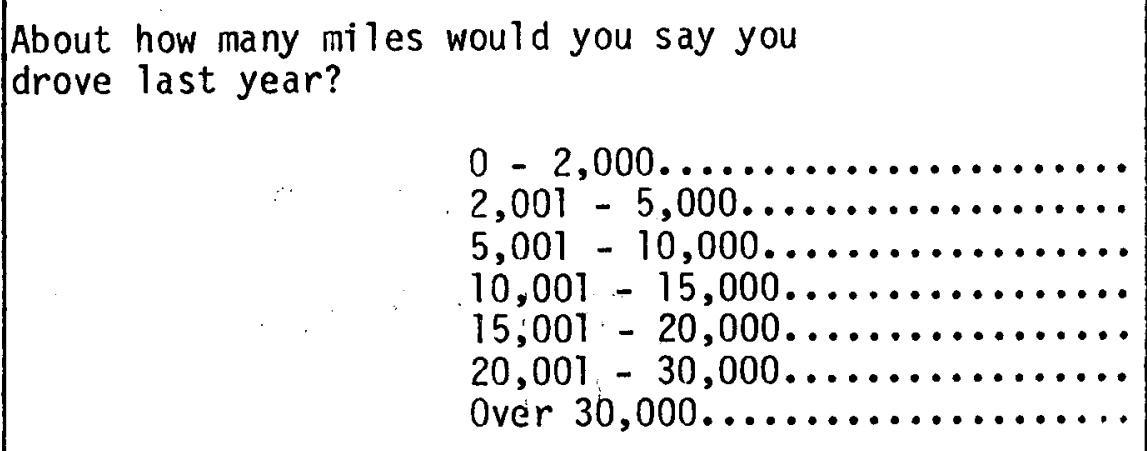 & $\begin{array}{r}15.9 \\
13.5 \\
21.8 \\
20.5 \\
10.3 \\
7.5 \\
9.0\end{array}$ \\
\hline AR & Telephone & 1979 & 400 & 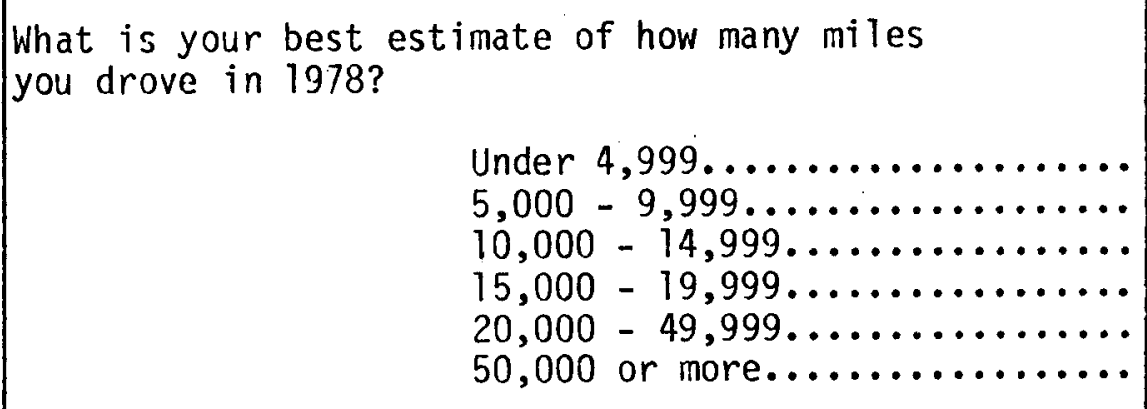 & $\begin{array}{r}39.3 \\
21.5 \\
17.8 \\
7.8 \\
12.5 \\
1.2\end{array}$ \\
\hline AK & Telephone & 1978 & 262 & 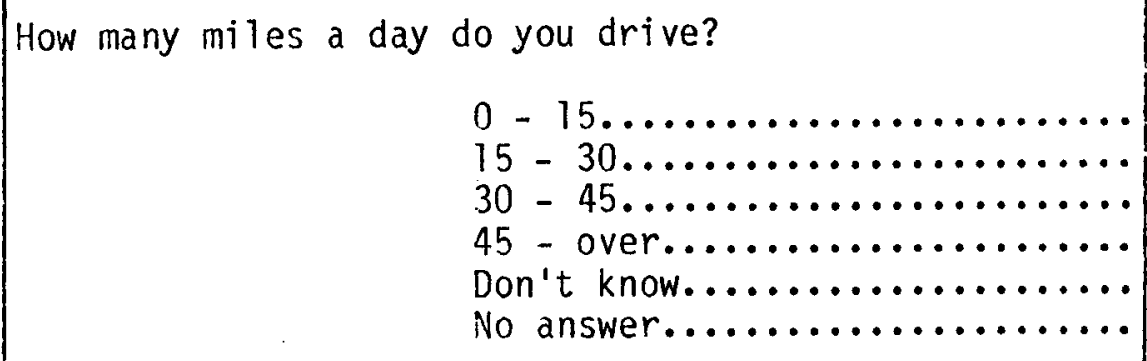 & $\begin{array}{r}51.5 \\
26.4 \\
11.7 \\
9.6 \\
0.4 \\
0.4\end{array}$ \\
\hline$A R$ & Tel ephone & 1979 & 403 & 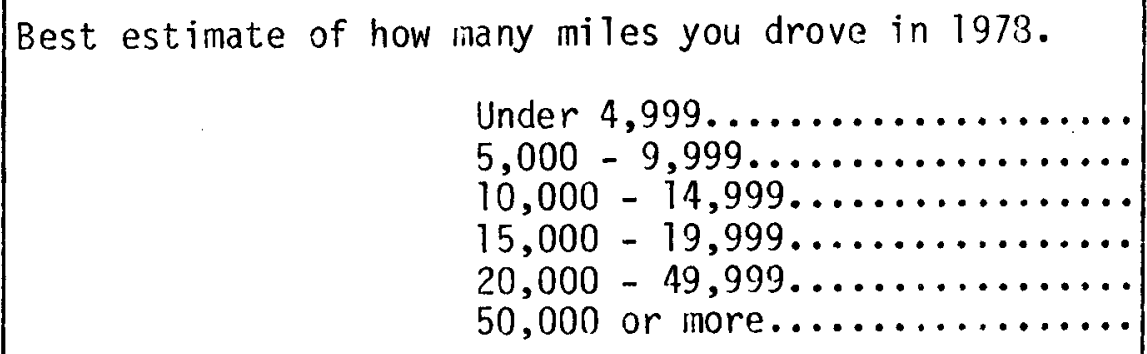 & $\begin{array}{r}32.0 \\
18.1 \\
25.1 \\
7.7 \\
15.1 \\
2.0\end{array}$ \\
\hline
\end{tabular}




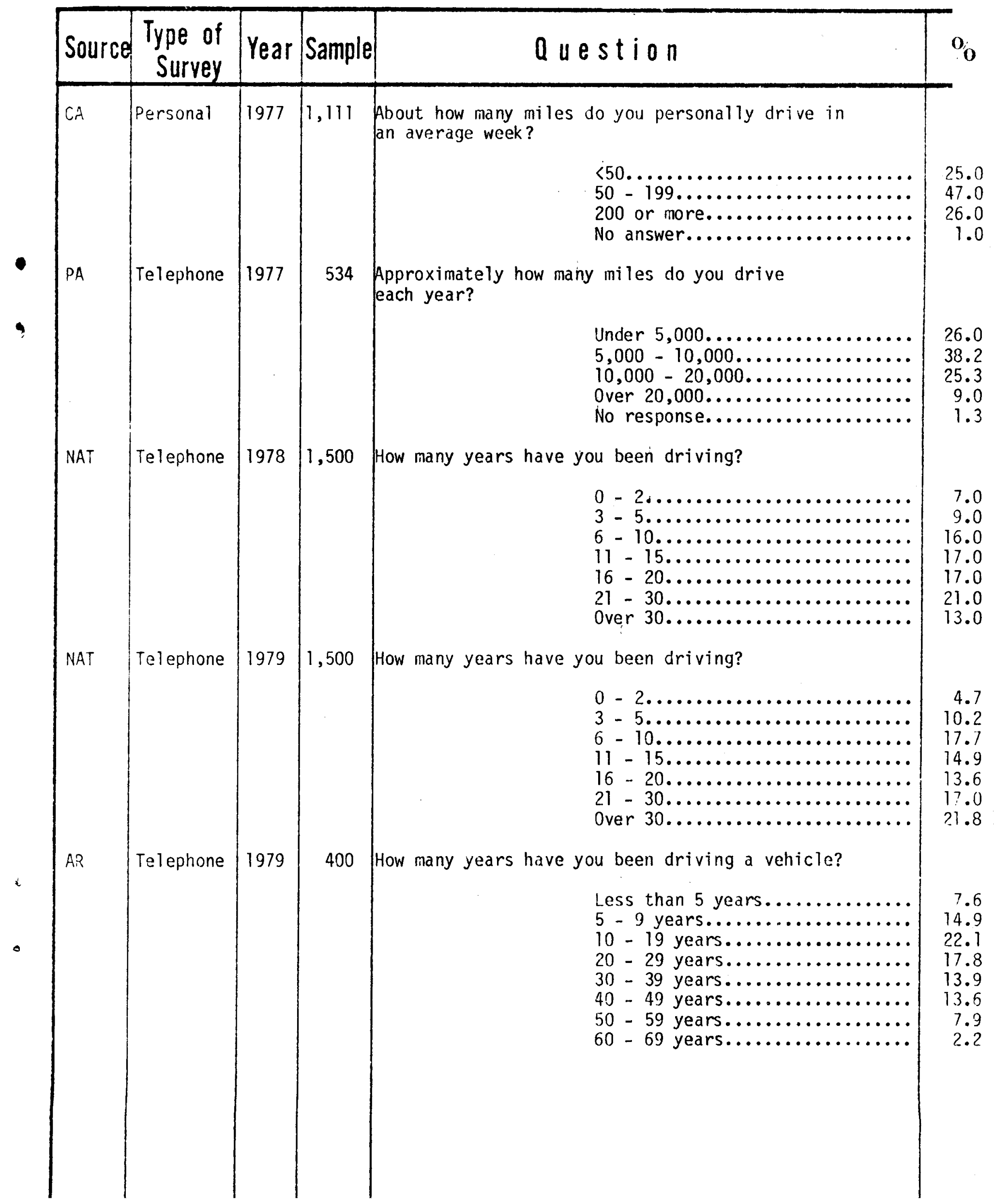




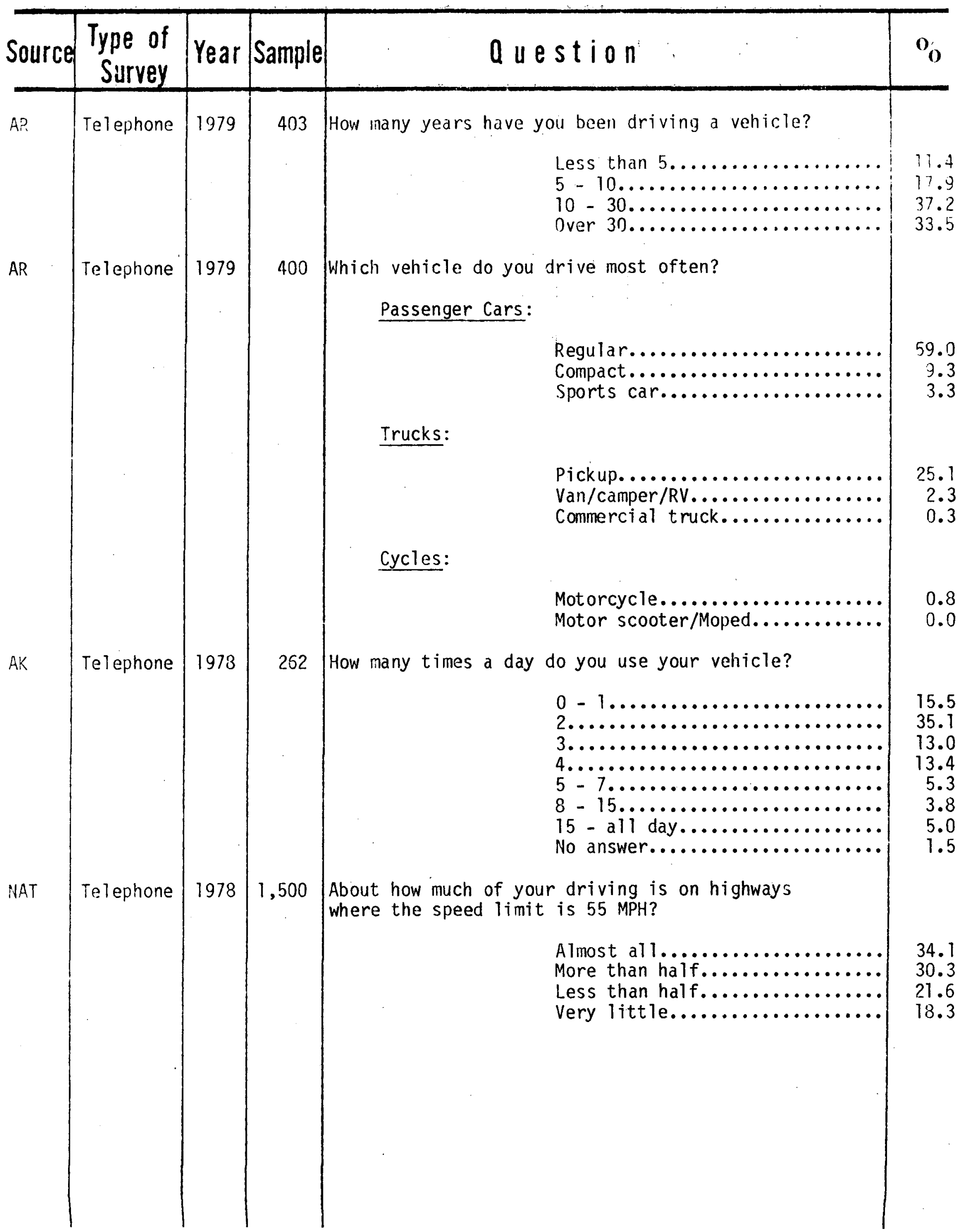




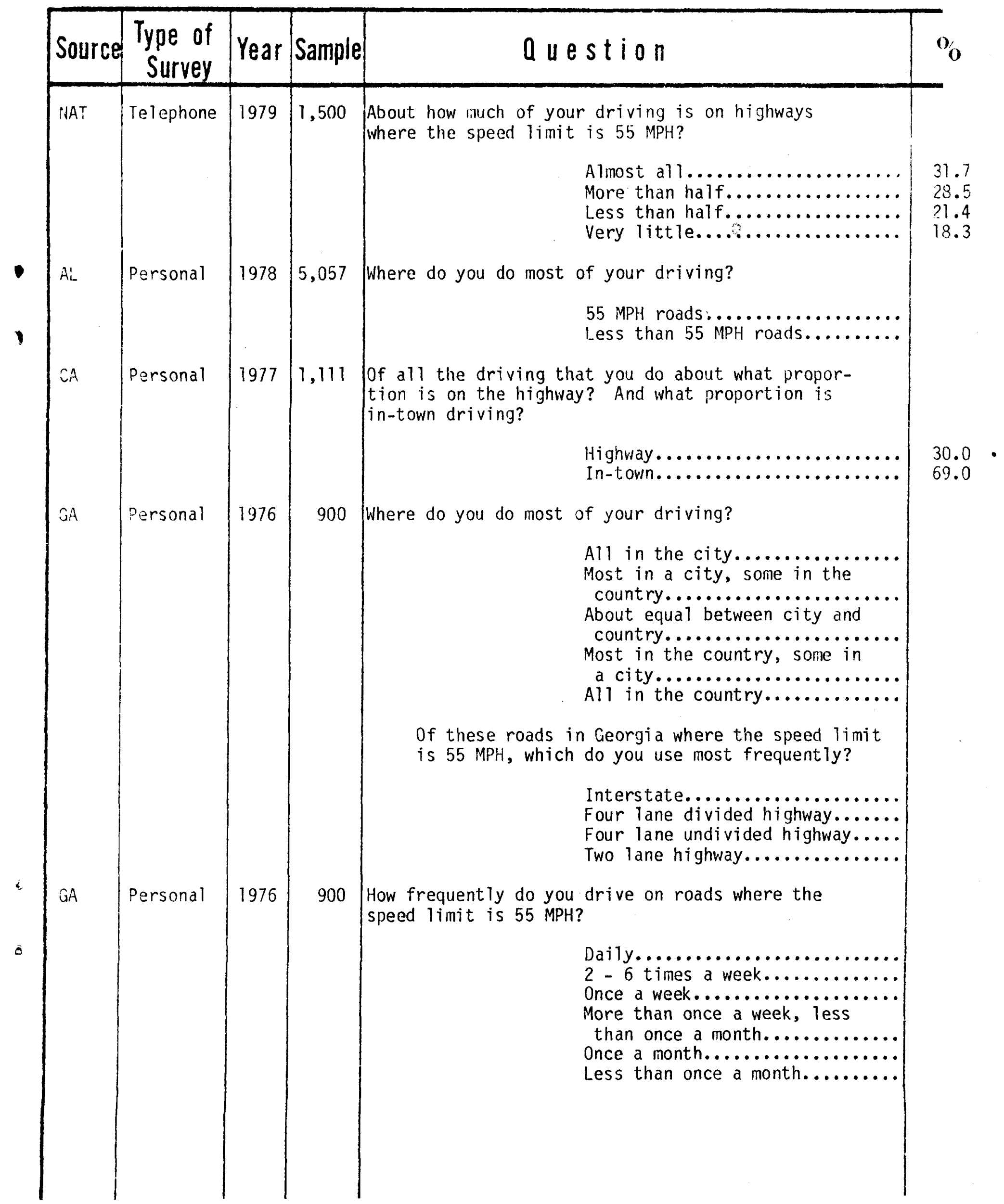




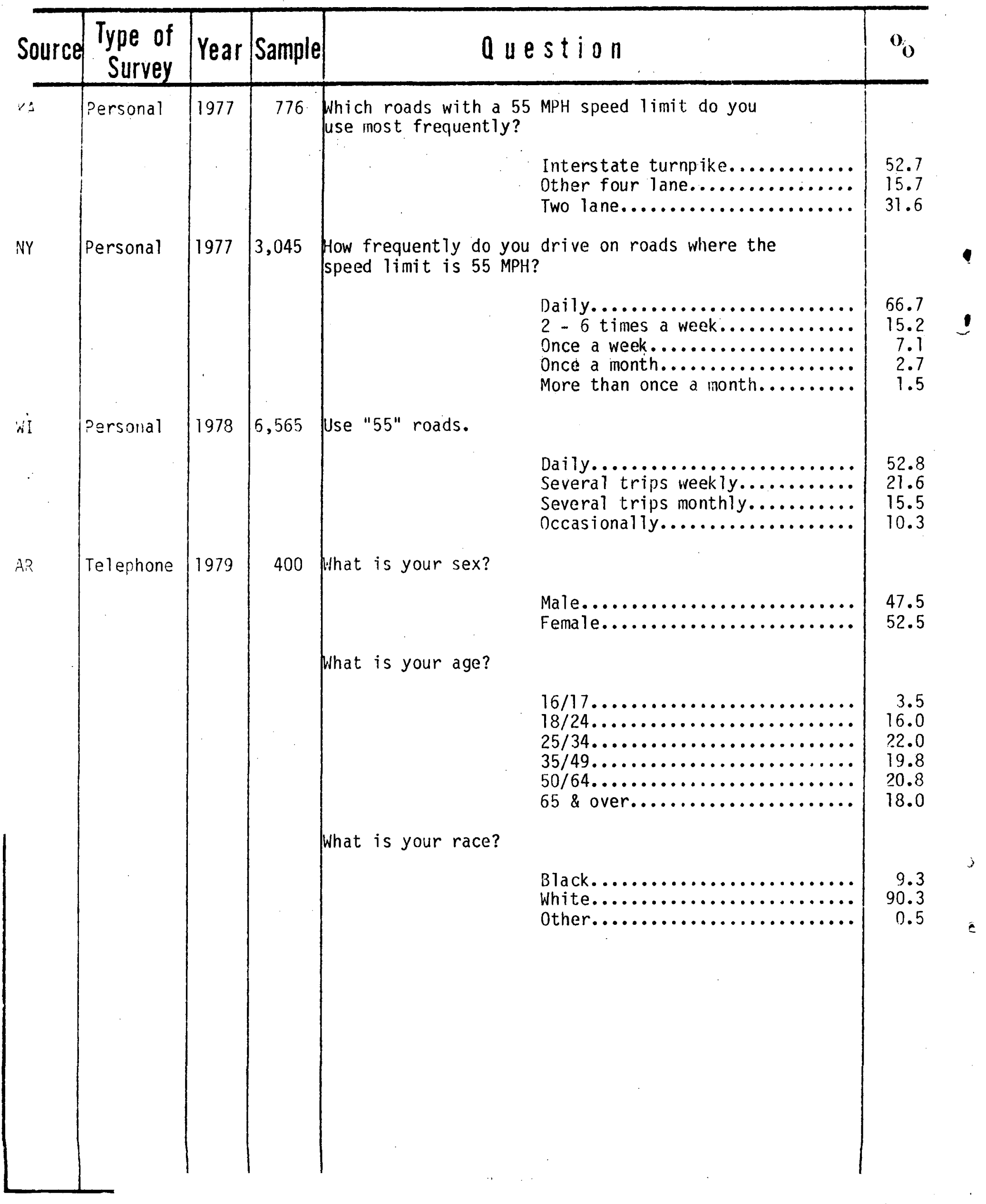




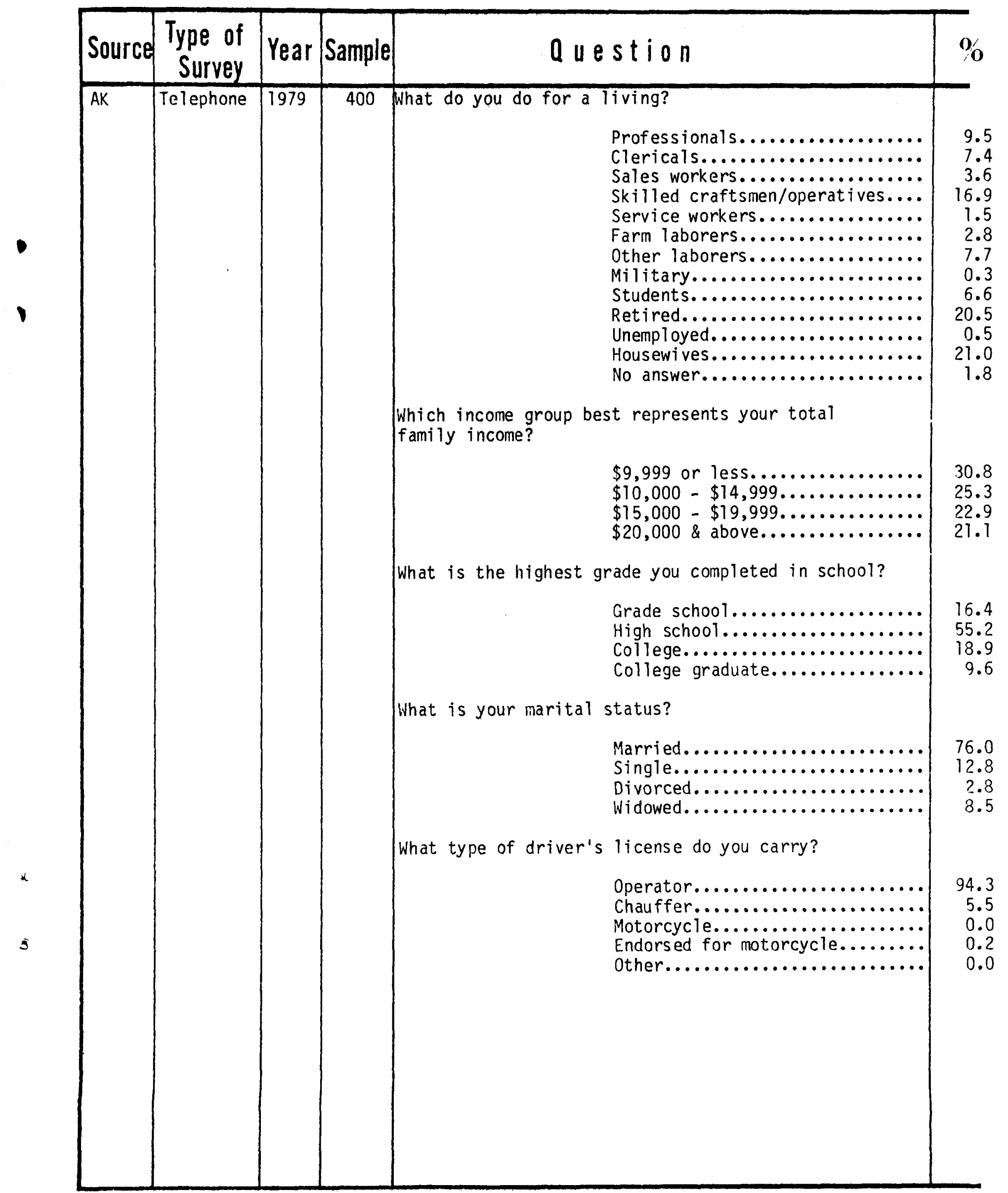




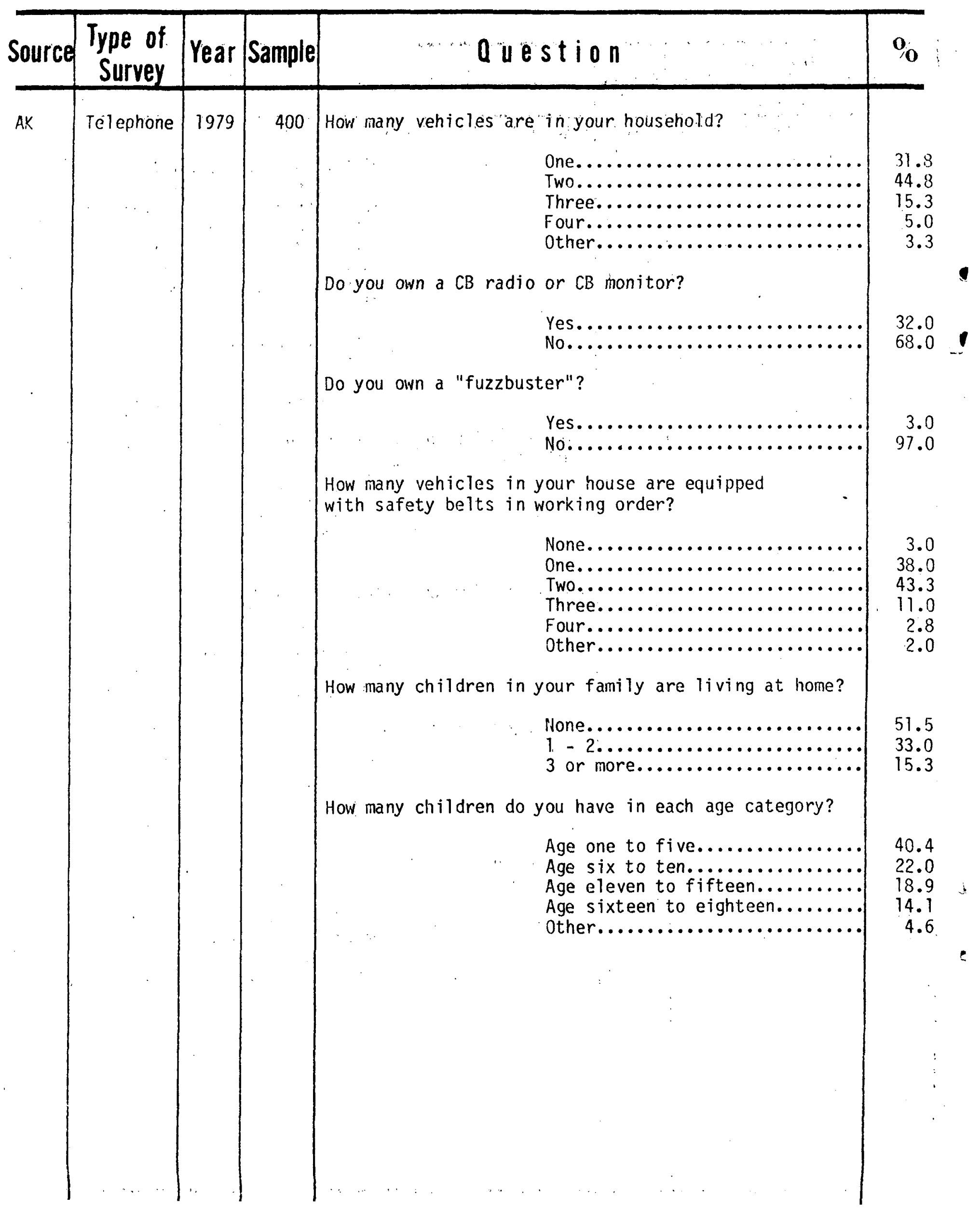

